\title{
Preoperational Subsurface Conditions at the Idaho Nuclear Technology and Engineering Center Service Wastewater Discharge Facility
}

S. L. Ansley

February 2002

Idaho National Engineering and Environmental Laboratory Bechtel BWXT Idaho, LLC 


\title{
Preoperational Subsurface Conditions at the Idaho Nuclear Technology and Engineering Center Service Wastewater Discharge Facility
}

\author{
Shannon L. Ansley
}

February 2002

Idaho National Engineering and Environmental Laboratory

Environmental Restoration Department

Idaho Falls, Idaho 83415

Prepared for the

U.S. Department of Energy

Assistant Secretary for Environmental Management

Under DOE Idaho Operations Office

Contract DE-AC07-99ID13727 


\begin{abstract}
The Idaho Nuclear Technology and Engineering Center (INTEC) Service Wastewater Discharge Facility replaces the existing percolation ponds as a disposal facility for the INTEC Service Waste Stream. A preferred alternative for helping decrease water content in the subsurface near INTEC, closure of the existing ponds is required by the INTEC Comprehensive Environmental Response, Compensation, and Liability Act (CERCLA) Record of Decision (ROD) for Waste Area Group 3 Operable Unit 3-13 (DOE-ID 1999a). By August 2002, the replacement facility was constructed approximately 2 miles southwest of INTEC, near the Big Lost River channel. Because groundwater beneath the Idaho National Engineering and Environmental Laboratory (INEEL) is protected under Federal and State of Idaho regulations from degradation due to INEEL activities, preoperational data required by U.S. Department of Energy (DOE) Order 5400.1 were collected. These data include preexisting physical, chemical, and biological conditions that could be affected by the discharge; background levels of radioactive and chemical components; pertinent environmental and ecological parameters; and potential pathways for human exposure or environmental impact.
\end{abstract}

This document presents specific data collected in support of DOE Order 5400.1, including: four quarters of groundwater sampling and analysis of chemical and radiological parameters; general facility description; site specific geology, stratigraphy, soils, and hydrology; perched water discussions; and general regulatory requirements. However, in order to avoid duplication of previous information, the reader is directed to other referenced publications for more detailed information. Documents that are not readily available are compiled in this publication as appendices. These documents include well and borehole completion reports, a perched water evaluation letter report, the draft INEEL Wellhead Protection Program Plan, and the Environmental Checklist. 


\section{CONTENTS}

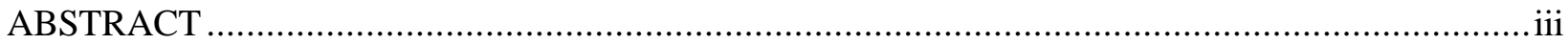

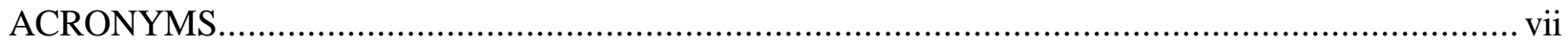

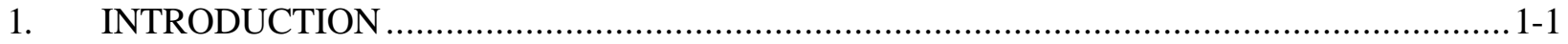

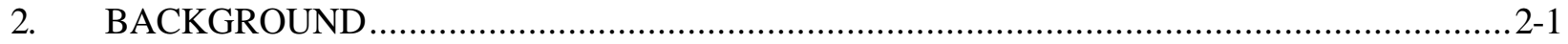

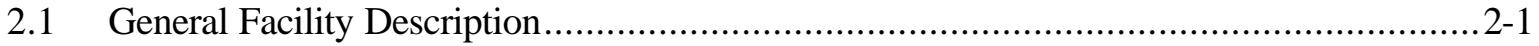

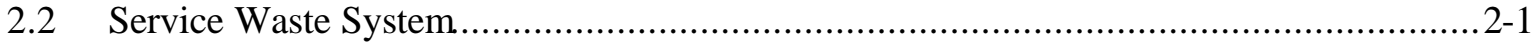

2.3 Waste Stream Water Quality and Flow Rates ................................................... 2-1

2.4 New Discharge Facility Description............................................................... $2-2$

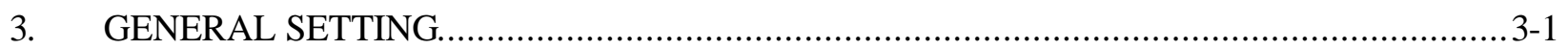

3.1 Site-Specific Physiography and Geology.......................................................... 3-1

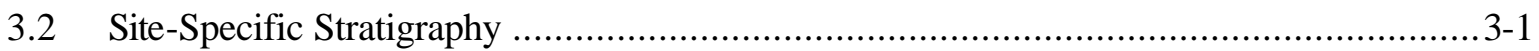

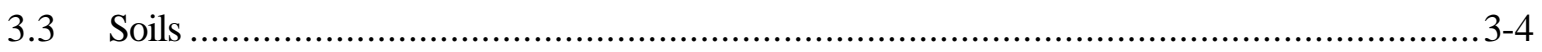

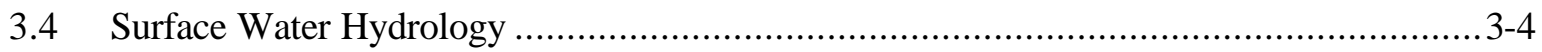

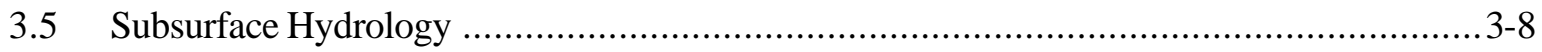

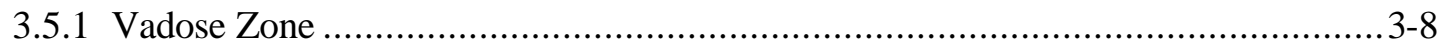

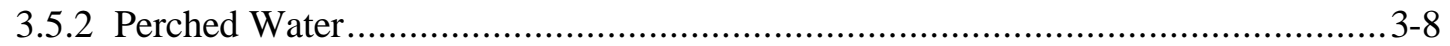

3.6 Snake River Plain Aquifer............................................................................ 3-10

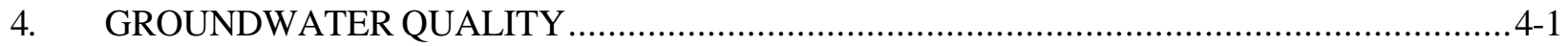

4.1 Historical Groundwater Quality Associated with INTEC and TRA..............................4-1

4.2 Pre-Operational Baseline Aquifer Monitoring and Sampling ..................................4-1

4.3 Perched Water Monitoring and Sampling ......................................................... $4-8$

4.4 Sample Analysis and Data Validation ...............................................................

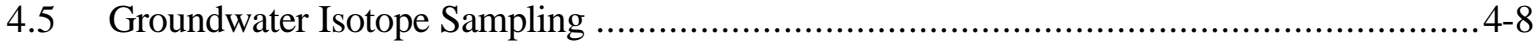

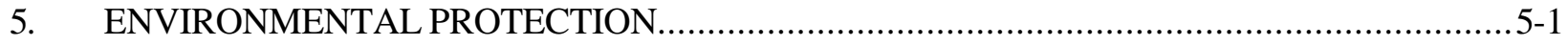

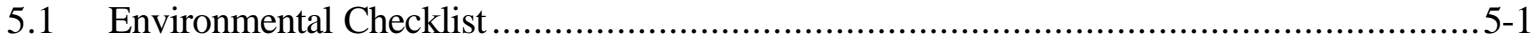

5.2 Wastewater Land Application Permitting ..................................................... 5-1 
5.3 Wellhead Protection and Capture Zones............................................................. 5-2

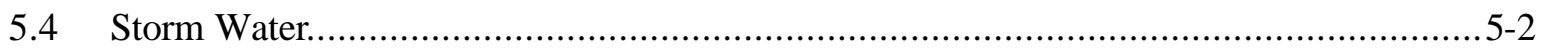

5.5 DOE Order 5400.1 Requirements ............................................................ $5-5$

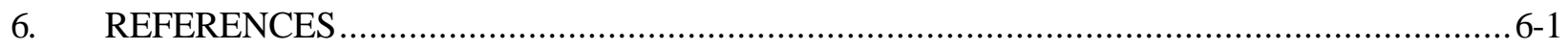

Appendix A-Well and Borehole Completion Reports........................................................

Appendix B-Letter Report - Perched Water............................................................................ B-1

Appendix C-Preoperational Groundwater Data..........................................................................

Appendix D_Wellhead Protection Plan ........................................................................................ D-1

Appendix E-Environmental Checklist........................................................................................ E-1

\section{FIGURES}

1-1. Location of INTEC, CFA, and the INTEC Service Wastewater Discharge Facility on the INEEL.

2-1. Diagram of the INTEC Service Wastewater Discharge Facility .........................................2-3

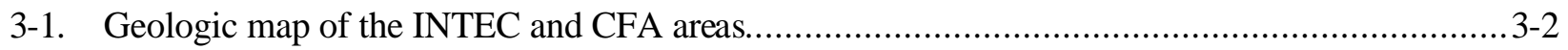

3-2. Surface topography of the INTEC and surrounding area .......................................... $3-3$

3-3. Channels and flow paths of the Big Lost River both off and on the INEEL........................... 3-5

3-4. USGS Big Lost River 100-year flood plain ........................................................... 3-7

3-5. Measured and WAG 3 OU 3-13-modeled extent of perched water beneath INTEC................... 3-9

4-1. Distribution of specific conductance of water from the SRPA at the INEEL, October 1995........4-2

4-2. Distribution of nitrate in water from the SRPA at the INEEL, October 1995 .........................4-3

4-3. Distribution of chloride in water from the SRPA at the INEEL, October 1995 .......................4-4

4-4. Distribution of tritium in water from the SRPA at the INEEL, October 1995 ........................ 4-5

4-5. Distribution of strontium-90 in water from the SRPA at the INEEL, October 1995.................4-6

4-6. Areal distribution of iodine-129 in the SRPA near the ICPP, 1990-91 .................................4-7

5-1. Wellhead protection zones for CFA and INTEC potable water wells .................................5-3

5-2. Big Lost River potential storm water drainage areas ............................................... 


\section{ACRONYMS}

\begin{tabular}{|c|c|}
\hline AEC & Atomic Energy Commission \\
\hline BBWI & Bechtel BWXT Idaho, LLC \\
\hline bls & below land surface \\
\hline BLM & U.S. Bureau of Land Management \\
\hline BLR & Big Lost River \\
\hline CERCLA & Comprehensive Environmental Response, Compensation, and Liability Act \\
\hline CFA & Central Facilities Area \\
\hline CFR & Code of Federal Regulations \\
\hline CRMO & Cultural Resource Management Office \\
\hline DCG & derived concentration guide \\
\hline DOE & U.S. Department of Energy \\
\hline DOE-ID & U.S. Department of Energy Idaho Operations Office \\
\hline EA & environmental assessment \\
\hline EBR-I & Experimental Breeder Reactor I \\
\hline EIS & environmental impact statement \\
\hline EPA & U.S. Environmental Protection Agency \\
\hline ESRP & Eastern Snake River Plain \\
\hline FFA/CO & Federal Facility Agreement and Consent Order \\
\hline HLWF & high-level waste treatment and interim storage facility \\
\hline $\mathrm{ICDF}$ & INEEL CERCLA Disposal Facility \\
\hline IDAPA & Idaho Administrative Procedures Act \\
\hline IDEQ & Idaho Department of Environmental Quality \\
\hline IDHW & Idaho Department of Health and Welfare \\
\hline INEEL & Idaho National Engineering and Environmental Laboratory \\
\hline INEL & Idaho National Engineering Laboratory \\
\hline
\end{tabular}




\begin{tabular}{|c|c|}
\hline INTEC & Idaho Nuclear Technology and Engineering Center \\
\hline ISFSI & Independent Spent Fuel Storage Installation \\
\hline $\mathrm{kPa}$ & kilopascal \\
\hline LET\&D & Liquid Effluent Treatment and Disposal \\
\hline LLWL & low-level waste landfill \\
\hline LMITCO & Lockheed Martin Idaho Technologies Company \\
\hline MCL & maximum contaminant level \\
\hline MCP & management control procedure \\
\hline MOA & memorandum of agreement \\
\hline NEPA & National Environmental Policy Act \\
\hline NHPA & National Historic Preservation Act \\
\hline NPL & National Priorities List \\
\hline NRF & Naval Reactors Facility \\
\hline NWI & National Wetland Inventory \\
\hline $\mathrm{OU}$ & operable unit \\
\hline RCRA & Resource Conservation and Recovery Act \\
\hline RI/BRA & Remedial Investigation, Baseline Risk Assessment \\
\hline RWMC & Radioactive Waste Management Complex \\
\hline SRPA & Snake River Plain Aquifer \\
\hline SWDF & Service Wastewater Discharge Facility \\
\hline SWPPP & Storm Water Pollution Prevention Plan \\
\hline SWPPP-CA & Storm Water Pollution Prevention Plan for Construction Activities \\
\hline SWPPP-IA & Storm Water Pollution Prevention Plan for Industrial Activities \\
\hline SWS & Service Waste System \\
\hline TAN & Test Area North \\
\hline TDS & total dissolved solids \\
\hline
\end{tabular}


TRA

Test Reactor Area

USACOE U.S. Army Corps of Engineers

USFWS U.S. Fish and Wildlife Service

USGS U.S. Geological Survey

UXO unexploded ordnance

WAG waste area group

WLAP Wastewater Land Application Permit 


\section{Preoperational Subsurface Conditions at the Idaho Nuclear Technology and Engineering Center Service Wastewater Discharge Facility}

\section{INTRODUCTION}

Beneath the Idaho Nuclear Technology and Engineering Center (INTEC), (administratively identified as Waste Area Group [WAG] 3), groundwater is recharged from surface water sources such as percolation ponds, sewage treatment lagoons, and occasionally, during wet years, from the Big Lost River. Other recharge sources may include lawn watering, storm water infiltration, and leaking underground pipelines containing nonhazardous and nonradioactive process water, fire water, steam condensate and radioactive liquid going to the tank farm. As a result, these water sources have produced the formation of several perched water zones beneath the INTEC at depths ranging from 31 to $128 \mathrm{~m}$ (100 to $420 \mathrm{ft}$ ) below land surface (bls). Many of the perched zones have been contaminated by downward transport of contaminants, primarily radionuclides (Sr-90, I-129, and tritium) from the overlying surface soils, and from two instances in which the INTEC injection well collapsed and service wastewater was released to the perched zones. Water flow in the perched water zones is primarily vertical and ultimately recharges the Snake River Plain Aquifer (SRPA). Consequently, perched water is one contaminant transport pathway between contaminated surface soils and the SRPA. In addition, contaminants already in the perched water are a secondary source of aquifer contamination.

The Service Waste System (SWS) and associated discharge into existing percolation ponds, located immediately south of the facility, account for a large percentage of water recharge to the subsurface. Therefore, the INTEC Comprehensive Environmental Response, Compensation, and Liability Act (CERCLA) Record of Decision (ROD) for WAG 3 Operable Unit (OU) 3-13 (DOE-ID 1999a) requires closure of the existing service waste percolation ponds as the preferred alternative for helping decrease water content in the subsurface. In response to this action, an alternative discharge option for the 1.5 to 2.5 million gallons per day of service wastewater was determined (DOE-ID 2000) and a new discharge location was identified (DOE-ID 1999b).

In August 2000, construction of a new service wastewater discharge facility began at a location approximately 2 miles southwest of the INTEC facility adjacent to the channel of the Big Lost River (Figure 1-1). Because groundwater beneath the INEEL is protected under Federal and State of Idaho regulations from degradation due to INEEL activities, including discharges associated with new ponds, preoperational information required by DOE Order 5400.1 was collected. These data include pre-existing physical, chemical, and biological conditions that could be affected by the discharge; background levels of radioactive and chemical components; pertinent environmental and ecologic parameters; and potential pathways for human exposure or environmental impact as a basis for determining the nature and extent of the subsequent routine operational and emergency effluent monitoring and environmental surveillance programs.

This document primarily presents and discusses the results of a baseline groundwater monitoring program initiated prior to startup operations at the service wastewater discharge facility. Additionally, the document is a compilation of all data collected and assembled in reference to the new facility. In order to avoid duplication of previous information and to maintain brevity, the reader will be directed to other referenced publications as needed. 


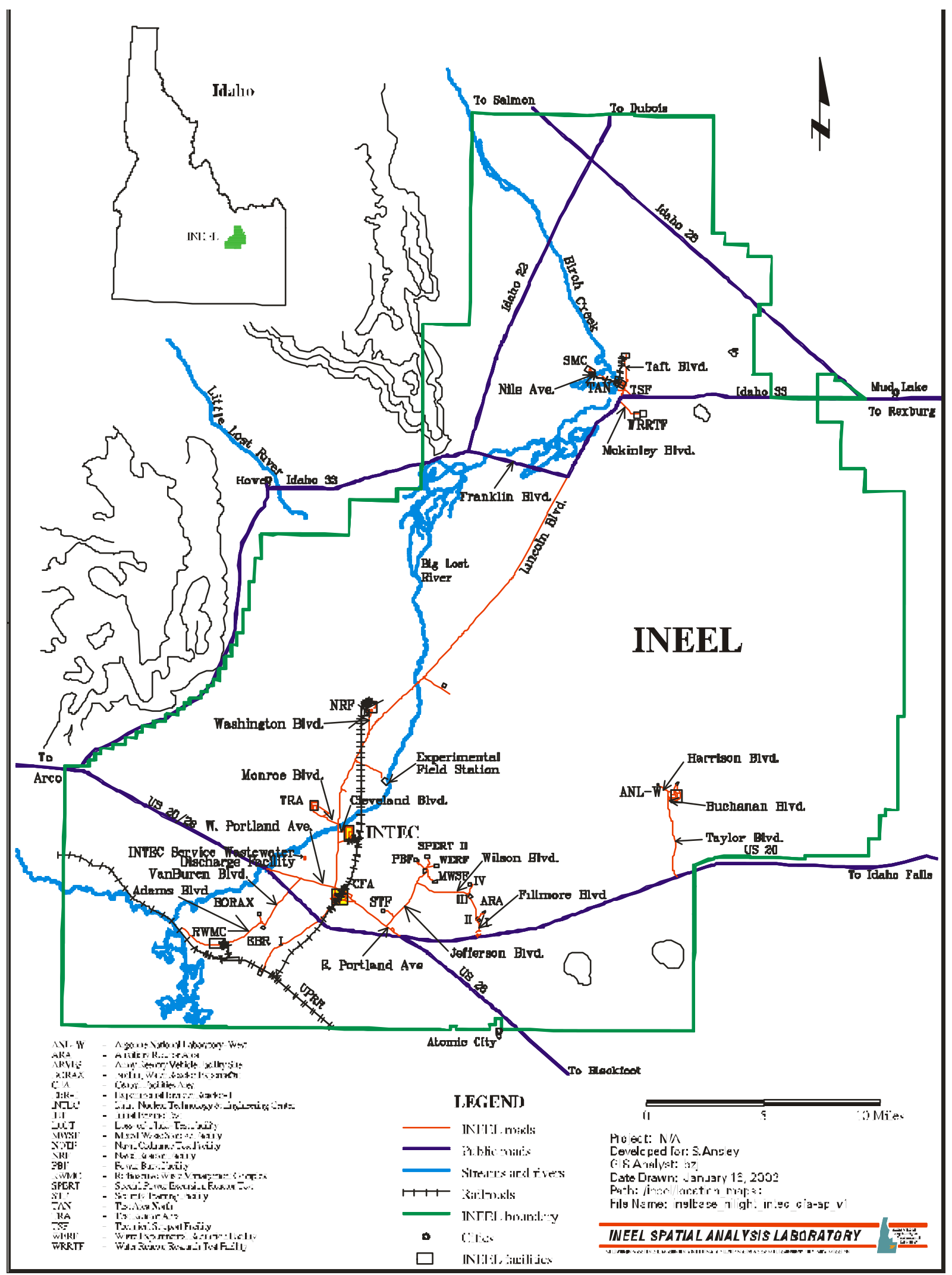

Figure 1-1. Location of INTEC, CFA, and the INTEC Service Wastewater Discharge Facility on the INEEL. 


\section{BACKGROUND}

\subsection{General Facility Description}

The INTEC, formerly the Idaho Chemical Processing Plant, is a multipurpose plant located on the INEEL, approximately $4 \mathrm{~km}(2.5 \mathrm{mi})$ north of CFA (Figure 1-1). Constructed in 1951, the INTEC initially contained all the facilities necessary to receive and store spent nuclear fuels, process the fuels to recover U-235, and handle waste generated by those functions. However, due to a mission change in 1992, the facility no longer recovers U-235. The current mission is to receive and temporarily store, prior to future disposition, spent nuclear fuel and waste fission products resulting from the spent fuel recovery processes. Research and development work is also conducted to develop and improve fuel management and waste processing technologies.

\subsection{Service Waste System}

The SWS, serving all major INTEC facilities, generates 5.6 to 9.4 million L/day (1.5 to 2.5 million gal/day) of process wastewater during normal operations. This process-related wastewater consists of steam condensate; noncontact cooling water; water treatment, demineralizer, and boiler blowdown wastewater; and other nonradioactive, nonhazardous liquids. Hazardous or radioactive wastewater from INTEC processes and laboratories are sent to either the low-level liquid waste evaporator or the high-level waste Tank Farm for treatment or storage. Sanitary wastes from restrooms and the cafeteria are discharged to the Sewage Treatment Plant located along the northeast boundary of the facility. Some sanitary wastes are directed to on-site septic tank systems.

Prior to discharge to the ponds, all service waste enters the final sampling and monitoring station (CPP-797) where it is measured for flow-rate, monitored for radioactivity, and sampled as prescribed by the State of Idaho Wastewater Land Application Permit (WLAP). Wastewater is then discharged to the percolation ponds.

Although radioactive wastewater systems have been isolated from the service waste stream since January 1993, systems containing liquid radioactive wastes are still present at the facility. The service waste stream is continuously monitored for radioactivity even though the risk of a failure of the protection systems that guard against an accidental discharge of radioactive wastes to the ponds is extremely low. A series of diversion mechanisms throughout the system are automatically triggered by gamma monitoring devices set to activate the system at a specified radioactivity.

\subsection{Waste Stream Water Quality and Flow Rates}

Current discharges from the SWS to the existing percolation ponds are regulated under a State of Idaho WLAP and reported annually in the WLAP Site Performance Reports for the INEEL to the Idaho Department of Environmental Quality (INEEL 2001, INEEL 2000a, LMITCO 1999, LMITCO 1998a, LMITCO 1997a, LMITCO 1996b). Discharges to the new ponds will be regulated under permit LA000130-03 issued September 10, 2001. Data confirming compliance with the permit will be reported in the annual Site Performance Report. Service wastewater samples are collected from the waste stream at CPP-797 (the final sampling and monitoring station) prior to discharge to the ponds. Between February and April 2000, the Environmental Restoration Organization collected confirmatory samples of service waste from CPP-797. Samples were analyzed for metals, nonmetals, I-129, Sr-90, gross alpha, gross beta, and gamma. The data are reported in EDF-ER-249. 


\subsection{New Discharge Facility Description}

The new INTEC Service Wastewater Discharge Facility (SWDF) (Figure 2-1) was designed to function in a similar manner to the old percolation ponds south of the INTEC facility. Essentially a rapid infiltration system, the new pond complex is composed of two cells excavated into the surficial alluvium and surrounded by bermed alluvial material. Each cell is approximately $96 \times 96 \mathrm{~m} \mathrm{(315} \mathrm{x} 315 \mathrm{ft})$ at the top of the berm and is 3.6 to $4.3 \mathrm{~m}$ (12 to $14 \mathrm{ft}$ ) deep. Each pond is designed to accommodate a continuous discharge of approximately 11 million liters per day ( 3 million gallons per day). Two sets of electric pumps transfer wastewater from CPP-797 to the discharge facility. 


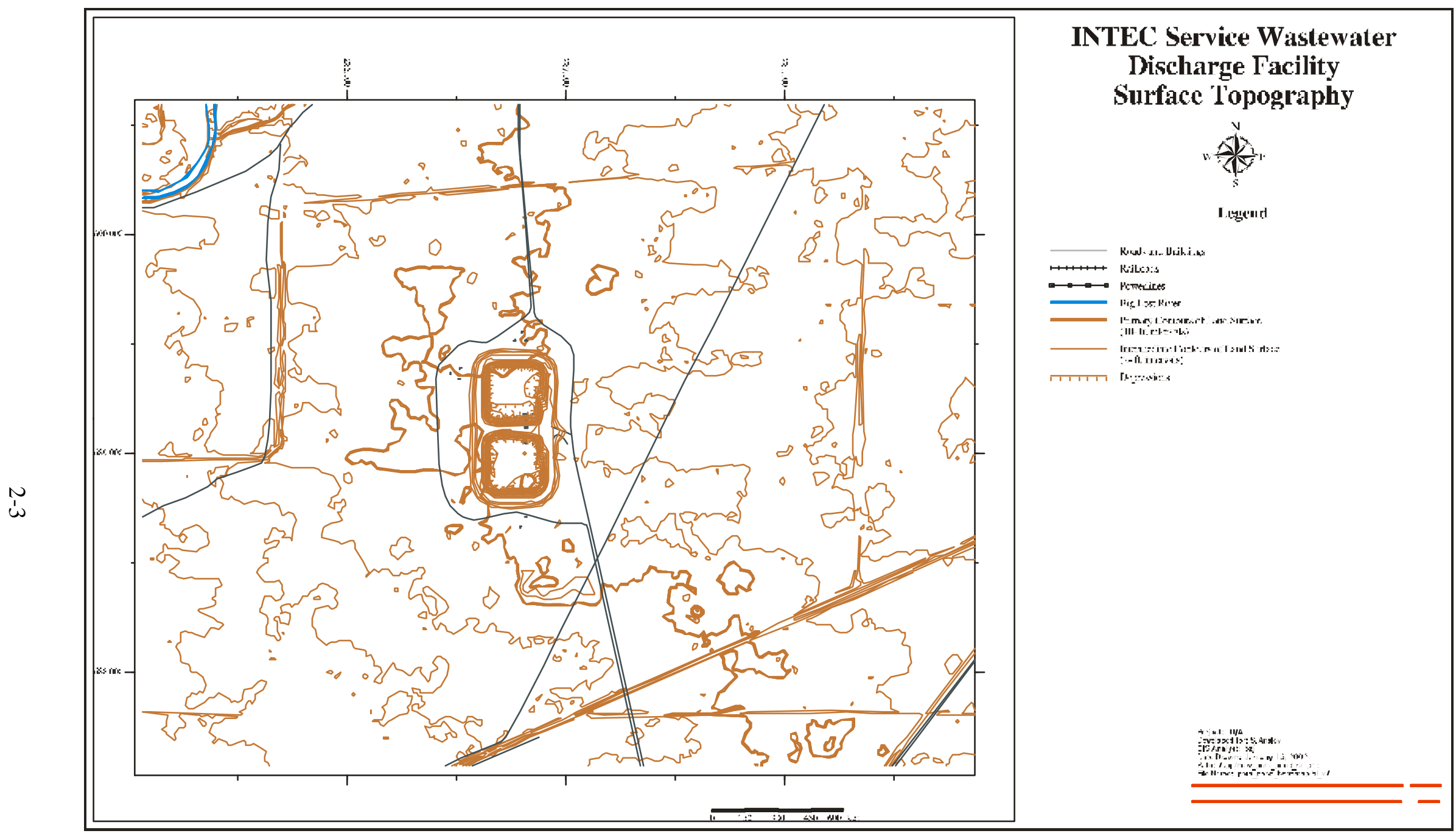

Figure 2-1. Diagram of the INTEC Service Wastewater Discharge Facility. 


\section{GENERAL SETTING}

This section briefly summarizes hydrogeologic and physical characteristics localized to the area of the INTEC SWDF. For more regional descriptions of geology, physiography, soils, surface and subsurface hydrology, meteorology and climatology, flora and fauna, archeological resources, demography and land use, and infrastructure, the reader is referred to the siting study done to select the location for the new facility (DOE-ID 1999b). Site-specific descriptions of similar information are presented in the following sections. Most of the information was obtained during drilling of shallow engineering boreholes, aquifer wells, and vadose zone instrumented boreholes.

\subsection{Site-Specific Physiography and Geology}

The new discharge facility is located in a flat-lying area just southeast of the Big Lost River channel in the south-central part of the INEEL. In this area, alluvial gravels from the Big Lost River cover a broad span approximately $6 \mathrm{~km}(3.7 \mathrm{mi})$ wide, bounded on the southeast and northwest by outcrops of basalt lava flows. The pond cells are actually sited on Late Pleistocene alluvial gravels on a low terrace above the Holocene floodplain (Figure 3-1). The Holocene floodplain, which lies between the INTEC and the TRA facilities, is characterized by numerous abandoned channels and possibly braided channels of the Big Lost River. The presently active channel, which has been dry at this location approximately 4 of the last 10 years, is incised into the Holocene floodplain deposits by about 1.5 to $2 \mathrm{~m}$ ( 4.9 to $6.5 \mathrm{ft}$ ), and is floored by light tan color sands and fine gravels. Air photographs of the Pleistocene terrace deposit on which the new facility is located shows no evidence of recent channels or braids of the river. A subdued meander-scroll topography is present over large areas of the Pleistocene surface (Figure 3-2). The ground surface is covered by sagebrush and the meander-scrolls are recognizable mainly from tonal anomalies on air photographs. Based on the degree of soil development and radiocarbon ages of sediments, the deposits that make up this surface were laid down during periods of high runoff during retreat of the most recent (Pinedale) glaciers, probably in the range of 15,000 to 20,000 years ago (Scott 1982, Ostenaa et al. 1999a).

The landforms adjacent to the Big Lost River alluvial deposits are dominated by lava flow surface morphology that has been subdued somewhat by deposition of loess and fine eolian sand in low areas and in the lees of ridges and hills. The lava flow surfaces are characte rized by rugged but low-relief topography. Due to deflation of parts of the surface during waning stages of volcanic activity, there are numerous closed basins separated by undeflated ridges. The largest of the basins (up to several tens of meters across) commonly contain thin playa deposits that cover the basin floors. The ridges are riddled with anastomosing fissures that are roughly parallel to the margins of the collapsed basins. Many of the outcrops show columnar jointing that produces a hexagonal or polygonal pattern of fractures on the outcrop surface.

\subsection{Site-Specific Stratigraphy}

At the new facility, the surficial sediments (Big Lost River alluvium) vary from 12 to $19.8 \mathrm{~m}$ ( 39.5 to $65 \mathrm{ft}$ ) thick and consist mostly of gravel, gravelly sands, and sands deposited by the Big Lost River during late Pleistocene time. The surficial sediments overlie an alternating sequence of basalt lava flows and interbedded sediments known as the Snake River Group, which may extend to a depth of 600 to $700 \mathrm{~m}$ (1,968 to 2,296 ft). Basalt lava flow groups make up at least $85 \%$ of the upper $213 \mathrm{~m}(700 \mathrm{ft})$ of stratigraphy near the facility, the remainder being sediment interbeds. The deepest borehole drilled at the pond location is $171 \mathrm{~m}(560 \mathrm{ft})$ below land surface. 


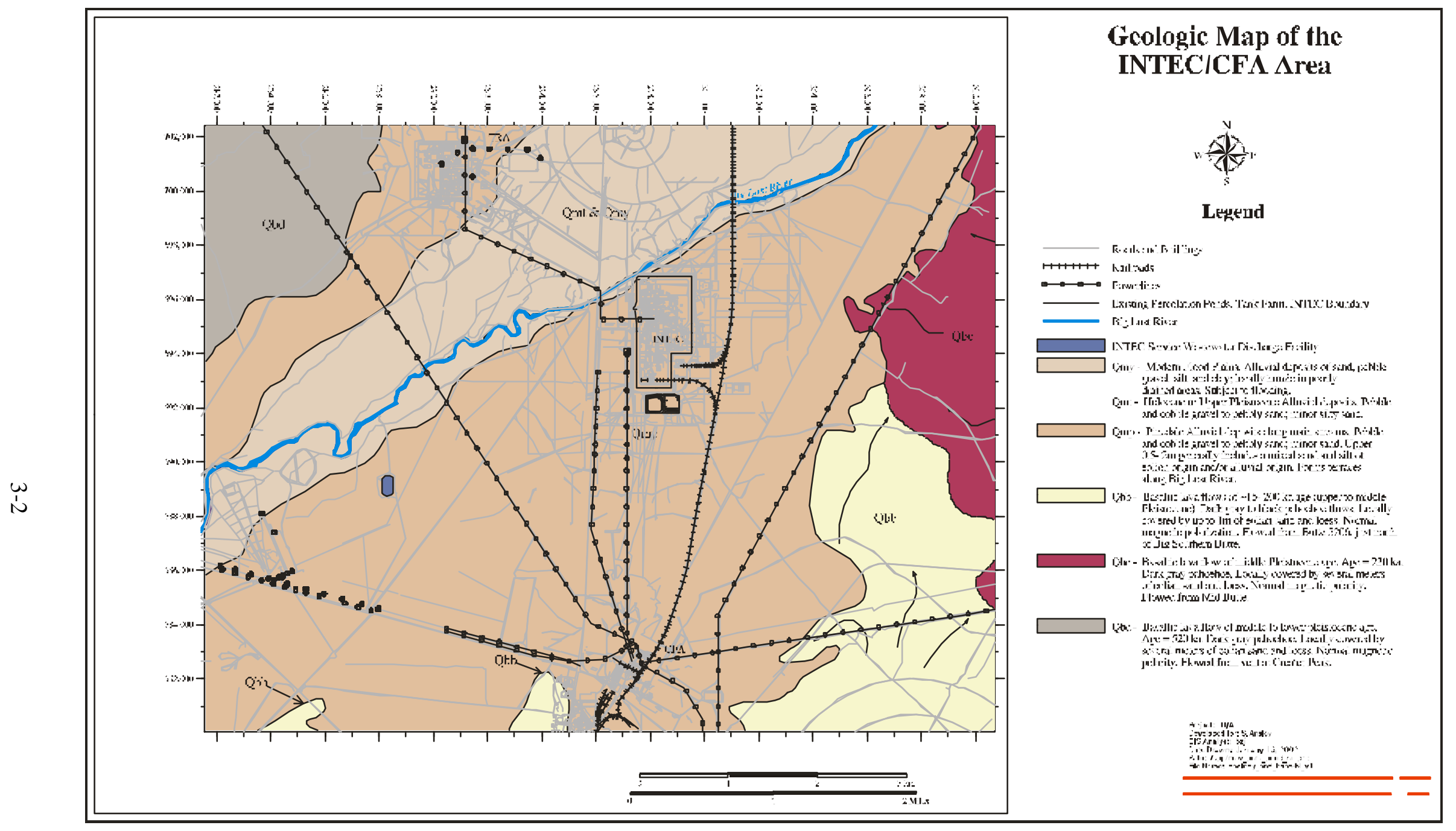

Figure 3-1. Geologic map of the INTEC and CFA areas. 


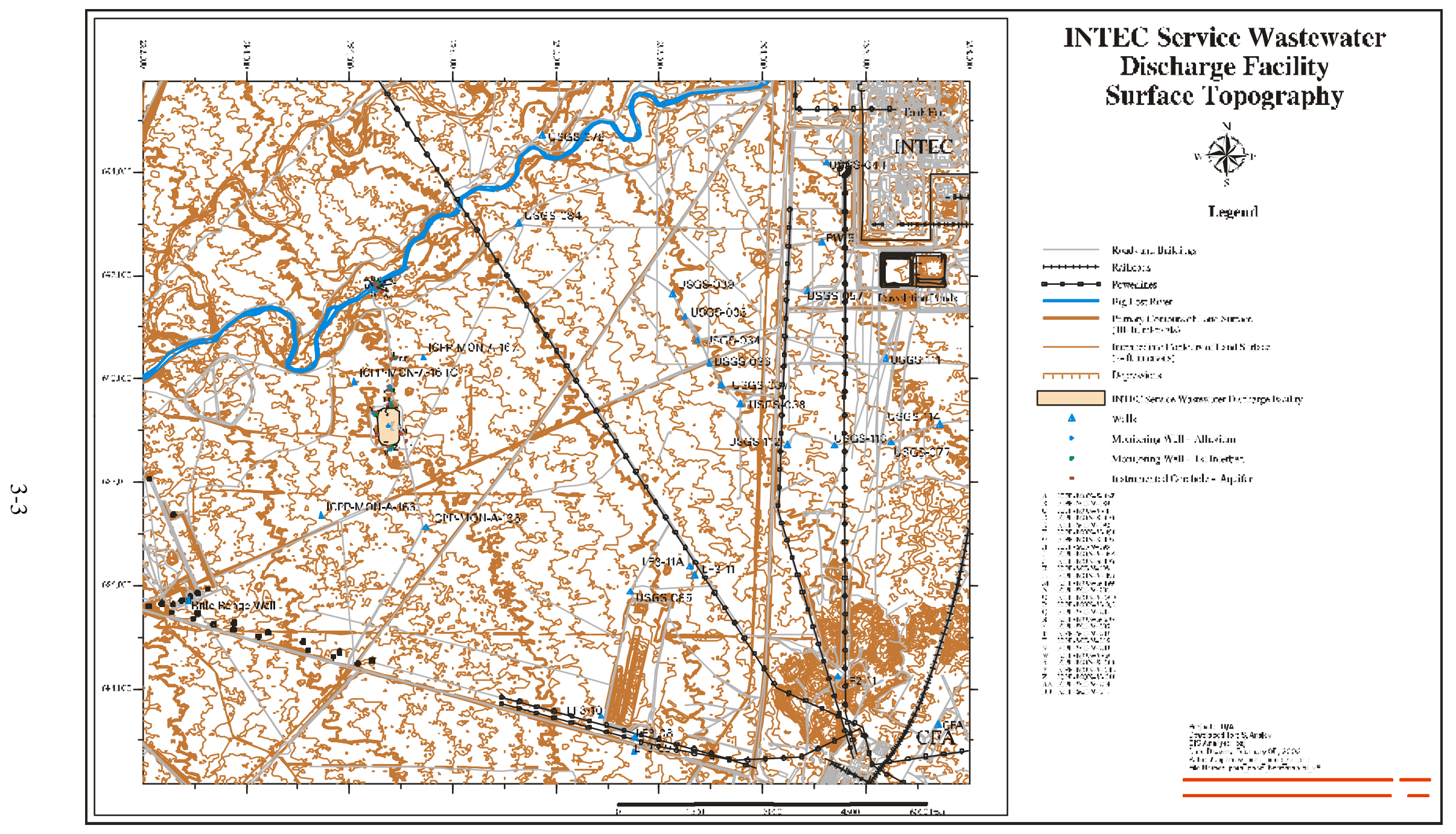

Figure 3-2. Surface topography of the INTEC and surrounding area. 
Although the surficial sediment at the new facility is composed of alluvial gravels, sedimentary interbeds within the Snake River Group are typically composed of silts, clayey silts, and sandy silts of both alluvial and eolian origin. Some of the deeper, thicker interbeds contain significant alluvial materials, including sands and gravels. Draft cross sections showing the positions and thickness of interbeds are presented in Appendix C. These sections show that the first major interbed occurs between depths of 37.5 to $44.5 \mathrm{~m}$ (123 to $146 \mathrm{ft}$ ) below land surface. A second thick interbed occurs between 55 and $72 \mathrm{~m}$ (181 and $236 \mathrm{ft}$ ) below land surface. Other interbeds occurring between 80-90 ft, 117-152 ft, 180-236 ft, and 320-330 ft bls may be continuous across the area covered by the well field. Additional interbeds observed in some boreholes between $275-308 \mathrm{ft}$ and $425-475 \mathrm{ft}$ bls may be discontinuous across the area. Deep cores collected from two holes by the U.S. Geological Survey have been logged for geophysical properties but not for lithologic identification. Interbed materials collected during all drilling operations have not been analyzed for hydraulic or physical properties.

Lithologic descriptions made during drilling activities indicate that the interbeds are composed of sand, silty sand, and some clay. Also throughout the stratigraphic section beneath the new facility are highly fractured and altered basalt rubble zones, sometimes infilled with fine-grained sediments, which may represent flow contacts.

\subsection{Soils}

Soils in the vicinity of the Big Lost River tend to be medium to coarse textured over gravel and are derived from alluvial deposits of the Big Lost River. These soils range from shallow, less than $51 \mathrm{~cm}$ (20 in.) to deep, more than $152 \mathrm{~cm}$ (60 in.). The cation-exchange capacity — an indicator of the ability of a soil to adsorb potential contaminants_-ranges from 0 to $30 \mathrm{meq} / 100 \mathrm{~g}$. Soils in the central portion of the INEEL tend to be moderately coarse-textured (from eolian sand) on basalt plains at depths ranging from shallow, less than $51 \mathrm{~cm}$ (20 in.) to deep, more than $152 \mathrm{~cm}$ (60 in.). The soils tend to have clay contents ranging from 2 to $35 \%$ and cation-exchange capacities ranging from 1 to $30 \mathrm{meq} / 100 \mathrm{~g}$. Soils in the southern portions of the site tend to be medium- to fine-grained (from loess) on basalt plains and also are shallow to deep.

\subsection{Surface Water Hydrology}

The INEEL stretch of the Big Lost River, the major natural surface water feature on the INEEL, is located approximately $1,500-2,000 \mathrm{ft}$ northwest of the new facility and is ephemeral with no recreational or consumptive uses of the water. Impounded and regulated by Mackay Dam (located approximately $81 \mathrm{~km}$ [50 mi] northwest of CFA in the Big Lost River Valley), water flows from the dam southeastward through the Big Lost River Valley and onto the INEEL (see Figure 3-3). Although streamflows are often depleted before reaching the INEEL, by irrigation diversions and infiltration losses along the river, flow actually reaching the INEEL is either diverted at the INEEL diversion dam or flows northward across the INEEL.

The INEEL Diversion Dam constructed in 1958 to divert high runoff flows from downstream INEEL facilities, consists of a small earthen dam and headgate that directs water from the main channel, through a connecting channel, and into a series of four natural depressions, called spreading areas located south and west of the RWMC. The capacity of the spreading areas is 58,000 acre- $\mathrm{ft}$ at an elevation of $1,539 \mathrm{~m}$ (5,050 ft) (McKinney 1985). Runoff from the Big Lost River has never been sufficient to exceed the capacity of the spreading areas and overflow the weir. 


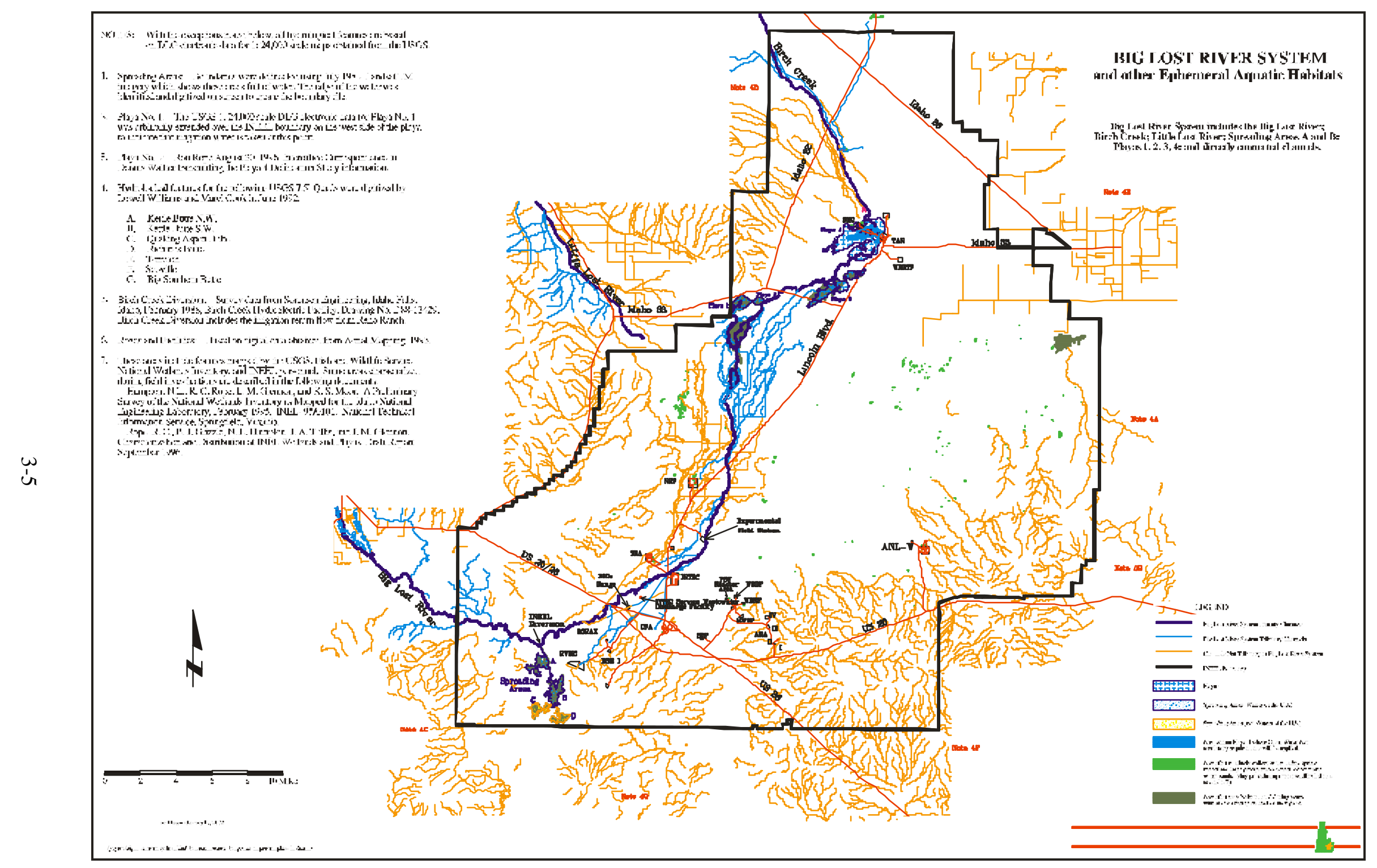

Figure 3-3. Channels and flow paths of the Big Lost River both off and on the INEEL. 
Big Lost River flow data for USGS flow monitoring stations at the closest downstream and upstream station from the location of the percolation ponds are 13132520 (near the INEEL Diversion) and 13132535 (at the Lincoln Boulevard Bridge). At station 13132520, flow was present every month from October 1998 through September 1999. Downstream at station 13132535, flow occurred in the BLR channel in all months except for the second half of December 1998 and January, February, and the first half of March 1999. This indicates channel infiltration occurred between the two stations during this period. For water year October 1999 to September 2000, station 13132520 showed flow in the channel in all months except the second half of May, and all of June, July, August, and September. At station 13132535, a similar flow pattern is observed with no flow in the channel for most of May and June through September.

Several studies have presented estimates of the potential magnitude of the 100-year flood for the Big Lost River, some of which are discussed below. The 100-year flood for the Big Lost River near Arco, a station $23 \mathrm{~km}$ (14 mi) upstream from the INEEL diversion dam, has an estimated magnitude of approximately 3,700 to 4,400 cfs based on a log-Pearson Type III distribution of historical stream gaging records (Tullis and Koslow 1983; U.S. Army Corps of Engineers (USACOE 1991); and Stone, Mann and Kjelstrom 1992). Another study used a log-Pearson Type III distribution for a station upstream of Mackay Reservoir combined with a regional regression approach for 22 subbasins and estimated a peak flow of 7,200 cfs for the 100-year flood for the Big Lost River at the Arco station (Kjelstrom and Berenbrock, 1996). The highest recorded flow at the Arco station was 1,890 cfs in July 1967. A recent study using paleohydrologic data collected from several stream reaches along the Big Lost River below the Arco station in combination with historical stream gage data from the Arco station, and a Bayesian flood-frequency analysis estimates a magnitude of 3,300 cfs for the 100-year flood for the Big Lost River at the Arco station (Ostenaa et al. 1999b). Ostennaa et al. also predicted peak flows on the Big Lost River with return periods of 500,1,000, and 10,000 years are 4,000, 4,400, and 5,300 cfs, respectively. These results suggest that exceedance of the estimated maximum capacity of the INEEL diversion dam of 9,300 cfs (Bennett 1986) has an extrapolated annual exceedance probability smaller than 0.00001 (or greater than the 100,000-year return period). Assuming a safeholding capacity of 5,000 cfs for the INEEL diversion dam the annual exceedance probability is 0.0002 (or a 5,000-year return period).

A USGS floodplain study (Berenbrock and Kjelstrom 1998) routed their conservatively high estimate of the 100-year peak flow (7,200 cfs) (Kjelstrom and Berenbrock 1997) downstream onto the INEEL (Figure 3-4). The flood-routing study did not include the INEEL diversion dam in the model simulation. The study assumes $1,000 \mathrm{cfs}$ of the peak flow will flow down the diversion channel and the remaining flow of $6,200 \mathrm{cfs}$ is routed downstream onto the INEEL using a one-dimensional code that does not account for infiltration, side or overbank losses.

Localized flooding occurred most recently in approximately 1962 when late winter frozen ground coupled with rapid snowmelt resulted in ponded water at the CFA facility. In order to drain the water and prevent a similar reoccurrence, a series of drainage ditches or canals were excavated to the north and northwest of the CFA. Many of these ditches are located within the vicinity of the new facility. 


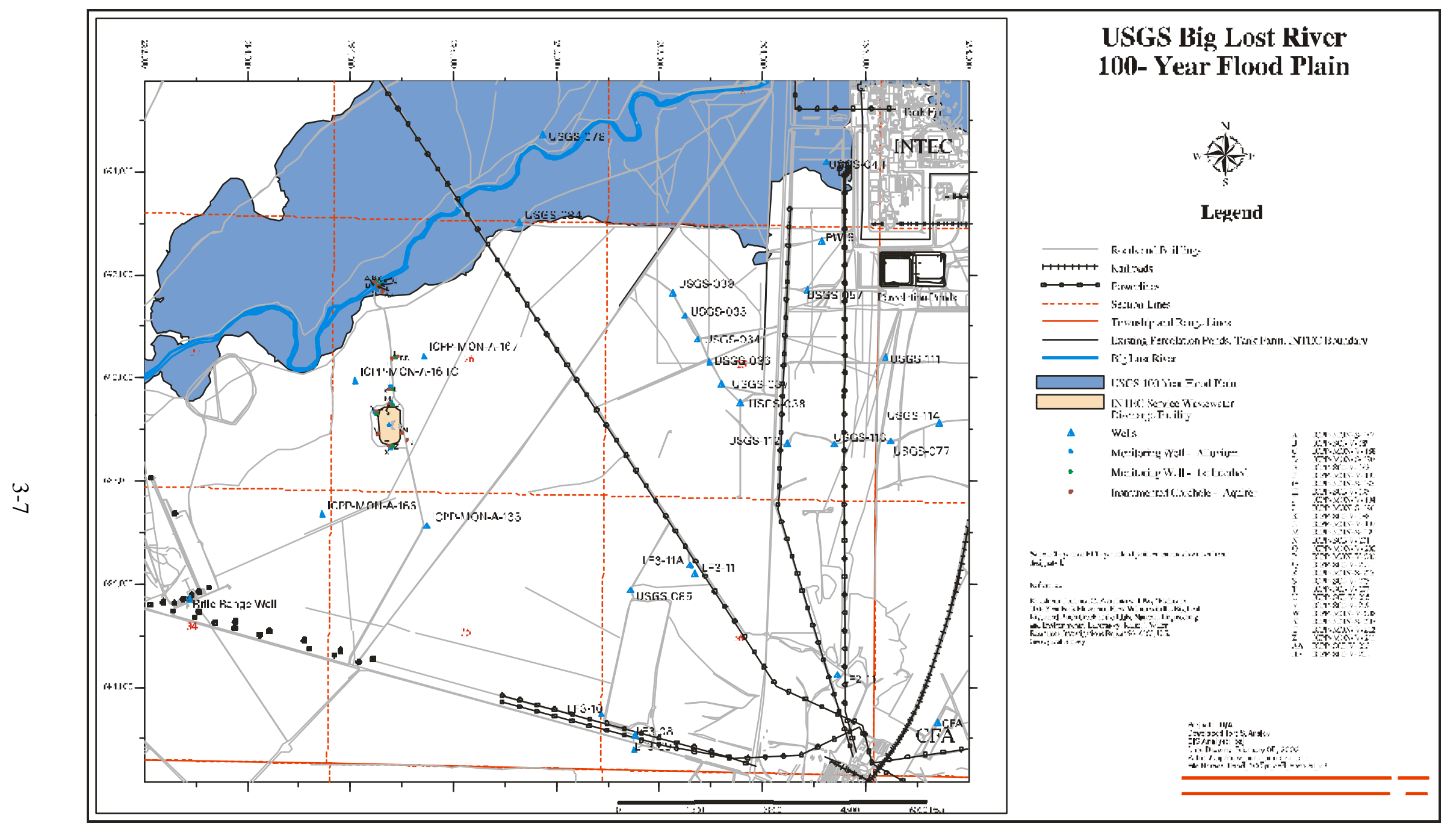

Figure 3-4. USGS Big Lost River 100-year flood plain. 


\subsection{Subsurface Hydrology}

\subsubsection{Vadose Zone}

Site-specific hydraulic properties of the vadose zone underlying the new facility are not characterized. However, some hydraulic properties will be obtained during research activities conducted at the INEEL Vadose Zone Research Park. The park encompasses the location of the INTEC SWDF and is coordinated by the INEEL Subsurface Science Program, the Manager of Environmental Monitoring, and the Geoscience organization. Currently, 26 monitoring points have been installed around the new facility; each designed as a monitoring well, an instrumented borehole, or a geophysical borehole. This network has the capability of measuring soil matric potential, soil temperature, soil moisture content, and $\mathrm{CO}_{2}$ content in soil gases at varying depths in the vadose zone. Additionally, some locations will have soil water sampling apparatus and some locations will collect electrical resistivity data. Background data are being collected prior to start-up of discharge of the ponds and will continue indefinitely. Appendix C details the locations and measurement capabilities of the wells associated with the Research Park.

\subsubsection{Perched Water}

Perched water bodies have formed beneath all INEEL infiltration ponds. The geohydrologic characteristics of the unsaturated zone underlying TRA, INTEC, TAN, and the RWMC differ with respect to basalt and sediment lithology, unit thickness, and physical orientation. These facilities also differ in the volumes of effluent discharged to the infiltration ponds, the sizes of the area over which recharge occurs, and the degree of saturation both horizontally and vertically. Although these differences exist, the features that control the formation of perched groundwater zones are common to the four facilities and to the new facility.

The discharge of wastewater into existing percolation ponds south of INTEC caused perched groundwater to form in the vicinity of the ponds (Figure 3-5). The lateral extent of this body approximates the pond boundaries and is dominated by vertical infiltration. Deeper perched water zones have been identified in the basalt at depths between 34 and $52 \mathrm{~m} \mathrm{(113} \mathrm{and} 170 \mathrm{ft})$ bls and 97 and $128 \mathrm{~m}(320$ and $420 \mathrm{ft})$ bls. The approximate extent of the 34-m (110-ft) perched zone is shown in Figure 3-5.

It is a basic assumption that perching of percolation pond infiltrate at relocated disposal ponds will occur similarly to that observed at the existing ponds. WAG 3 OU 3-13 modeling for a $1.5 \mathrm{mg} /$ day discharge to the existing ponds predicts the lateral spread of $100 \%$ saturation to be $549 \mathrm{~m}(1,800 \mathrm{ft})$ from the center of the existing ponds (Figure 3-5). The estimated 90\% saturation boundary for the same discharge volume is $1,555 \mathrm{~m}(5,100 \mathrm{ft})$. For the new percolation ponds, it is assumed that there will be no impact to the existing perched water at INTEC from new pond discharges because: (1) the OU 3-13 WAG 3 model is extremely conservative, (2) there is a long time interval before the new ponds will receive $3 \mathrm{MG}$ /day of waste water, (3) the measured perched water extent beneath the existing ponds is smaller than the OU 3-13 modeled extent, and (4) there is a 3-km (2-mi) separation between the existing ponds and the new ponds. For the purpose of estimating the extent of perched water at the new facility, no measured or modeled predictions of perched water extent exist, especially regarding impacts from the Big Lost River (BLR) during channel flow. However, within boreholes ICPP-MON-A-164 (A, B, and C) and ICPP-MON-A-167, cascading water was observed in video logs of the holes after drilling. At their shortest distance, these wells are located approximately 800 and $1700 \mathrm{ft}$, respectively, from the BLR channel. Video logs taken in wells ICPP-MON-A-164B in March 2000 show water entering the borehole at approximately $170 \mathrm{ft}$ bls and cascading downward, obscuring observation of the remainder of the hole. Volume measurements of water entering these boreholes were not made. (USGS measurements of BLR 


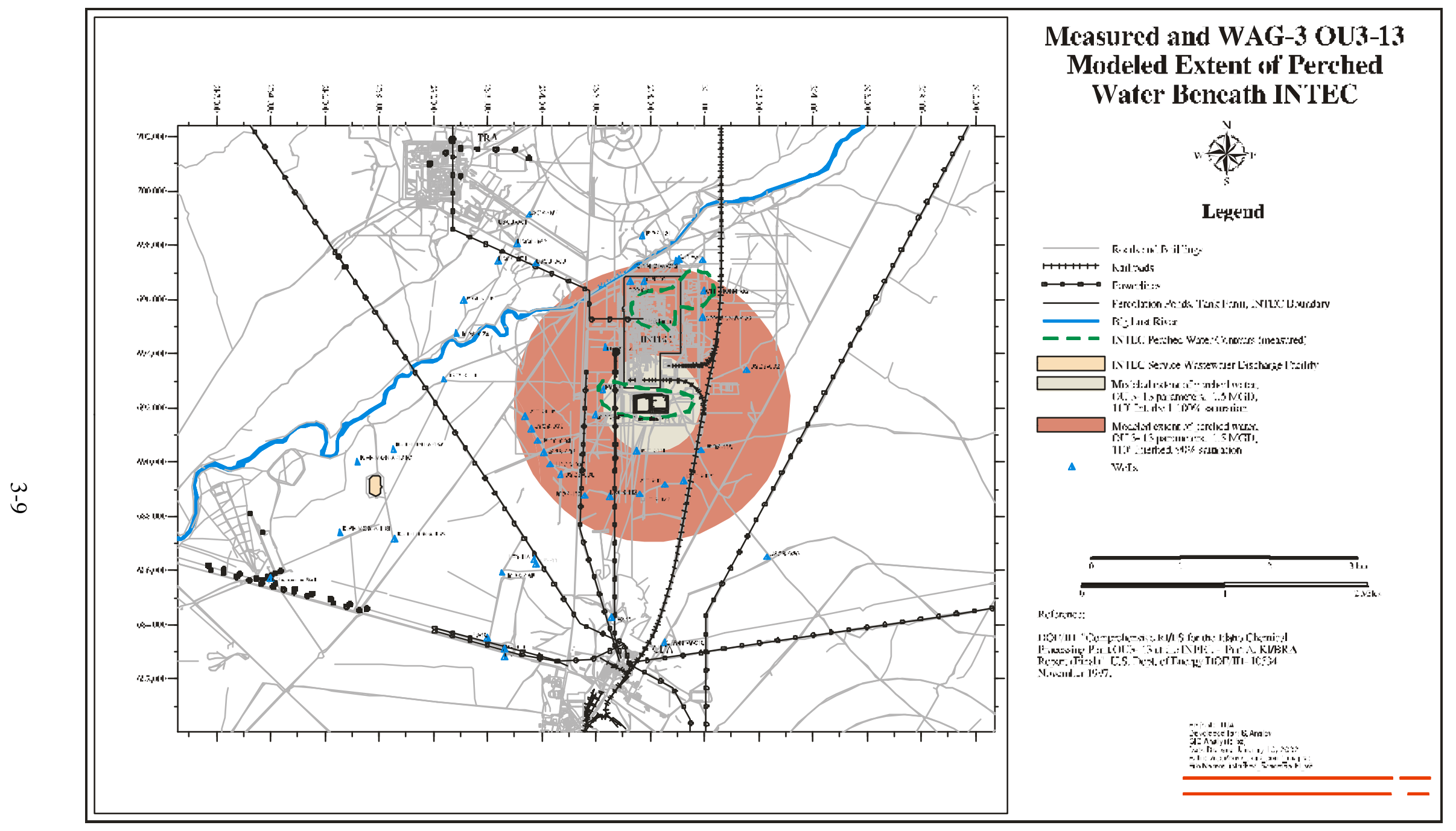

Figure 3-5. Measured and WAG 3 OU 3-13-modeled extent of perched water beneath INTEC. 
flow during this month was a maximum of $68 \mathrm{cfs}$ at the INEEL diversion station \#13132520 and a maximum of $55 \mathrm{cfs}$ at station \# 13132535 at the Lincoln Boulevard bridge, downstream of the percolation ponds. These flow measurements represent the maximum flow in the BLR channel during the entire water year October 1999 to September 2000.) A video log taken in June 2000 when there was no flow in the BLR channel, shows water still cascading down the borehole but from a lower level, $235 \mathrm{ft}$ bls. In the video log of ICPP-MON-A-167 taken also in June 2000, water can be observed cascading down the borehole, apparently originating at a depth of $198 \mathrm{ft}$ bls. From these data, it can be predicted that perched water originating from BLR flow can extend as far as $1700 \mathrm{ft}$ (and probably more) from the center of the channel. Information is not available to predict the drainage rate of any perched water body. In dry water years such as those observed from 1988 through approximately 1994, one could assume that after an unknown period of time, perched water below the BLR would dissipate and drain as long as flow in the channel remains zero. Similarly, perched water originating from discharges to the percolation ponds will dissipate and drain at some unknown rate upon cessation of discharge.

\subsection{Snake River Plain Aquifer}

The SRPA, one of the most productive aquifers in the United States (Lindholm 1981), was classified as a sole-source aquifer by the EPA in 1991. The aquifer flow embedded within the basalt stratigraphy of the Snake River Plain is governed by the structure of individual basalt layers. Horizontal movement of water within the aquifer, the predominant movement direction, is aided by hydraulically connected interflow zones created by the upper vesicular zone of a basalt flow coupled with the fractured and often rubbly substratum of the overlying flow. Vertical movement of water, controlled by pore size, fracture size, and fracture density, is generally limited within the dense interior elements of a flow. Fracture joints in the central portion of the lava flow are typically vertical in orientation. As discussed earlier, although these vertical fractures are believed to serve as the primary means for vertical groundwater movement, they may actually lead to little vertical or horizontal movement of groundwater flow due to sedimentary and chemical infilling of fractures and alteration zones. Further contributing to the reduction of vertical flow is the presence of sedimentary interbeds. These interbeds, typically composed of fine-grained, clayey materials, have hydraulic conductivities 3 to 5 orders of magnitude lower than that of the surrounding fractured basalt. Detailed regional descriptions of the SRPA can be found in Holdren et al. 1997 and DOE-ID 1997.

Groundwater beneath the new facility is approximately $152 \mathrm{~m}(500 \mathrm{ft})$ bls. Regional and local groundwater flow is south-southwest. No estimates of local hydraulic conductivity have been made, however, the hydraulic conductivity of the SRPA near INTEC was estimated using the transmissivity values reported by Ackerman (1991) and the saturated thickness of the open interval of the well. The estimation of hydraulic conductivity was based on the assumption that the wells fully penetrate the saturated thickness of the aquifer. Hydraulic conductivities range five orders of magnitude with a maximum of $3.0 \times 10^{3} \mathrm{~m} /$ day $\left(1.0 \times 10^{4} \mathrm{ft} /\right.$ day $)$ at Well CPP-3 and a minimum of $3.0 \times 10^{-2} \mathrm{~m} /$ day $\left(1.0 \times 10^{-1} \mathrm{ft} / \mathrm{day}\right)$ at Well USGS-114. The average hydraulic conductivity within the immediate vicinity of INTEC is $4.0 \times 10^{2} \pm 7.9 \times 10^{2} \mathrm{~m} /$ day $\left(1.3 \times 10^{3} \pm 2.6 \times 10^{3} \mathrm{ft} /\right.$ day $)$. Using the average hydraulic conductivity—a hydraulic gradient of $1.2 \mathrm{~m} / \mathrm{km}(6.3 \mathrm{ft} / \mathrm{mi})$ (Cecil et al. 1991) — and an effective porosity of $10 \%$, the calculated seepage velocity near the INTEC is approximately $3 \mathrm{~m} /$ day $(10 \mathrm{ft} /$ day). Groundwater flow velocitie s have also been estimated by observation of tritium concentration changes in wells downgradient of the INTEC injection well. 


\section{GROUNDWATER QUALITY}

\subsection{Historical Groundwater Quality Associated with INTEC and TRA}

The U.S. Geological Survey office based at the INEEL has historically collected groundwater samples to monitor the concentrations and movement of contaminants in the aquifer that resulted from operations at various facilities. These data can be found in USGS publications or acquired from the Environmental Restoration Information System database. Groundwater data specific to INTEC are also contained in the RI/BRA for WAG 3 OU 3-13 (DOE-ID 1997).

Figures 4-1 through 4-6 present the distribution of contaminant plumes for specific conductance, nitrate, chlor ide, tritium, strontium-90, and iodine-129 in the Snake River Plain Aquifer as of October 1995. As shown, contaminant plumes originating from both TRA and INTEC, as a result of past disposal practices, encompass or approach the location of the new discharge facility.

\subsection{Pre-Operational Baseline Aquifer Monitoring and Sampling}

Groundwater samples were collected from aquifer wells ICPP-MON-A-164C, -165, and -167 in November 2000 and January, February, and March 2001. Well ICPP-MON-A-166 was sampled in March and May 2001 and an additional sample was collected from ICPP-MON-A-167 in May 2001. Because the new facility was located in a previously undisturbed area where no known waste disposal has occurred, a comprehensive groundwater sampling program was required in order to assess the impact of the new facility on existing groundwater quality. The goal of the program was to collect four rounds of samples from each well prior to the initial discharge of wastewater to the newly constructed ponds. Except for well ICPP-MON-A-166, the program goal was met. Only two rounds of samples were collected from this well due to pump installation problems, inadequate generator capability, and other minor logistical problems.

Sampling plan SAP-EM-GW-3, Revision 0, (INEEL 2000b) outlines the requirements for sampling and the constituents analyzed. Data obtained from the sampling activities are presented in Appendix C.

In general, data results show no abnormal concentrations or occurrences of contaminants expected to be within the contaminant plumes from TRA and INTEC. All results are consistent with USGS data.

Volatile organic compounds were not detected above their respective maximum contaminant levels (MCLs). The only unflagged, valid detection of a VOC (three orders of magnitude below the MCL) was for toluene in the March 2001 sample from well ICPP-MON-A-166. Toluene was not detected in the associated trip blank. Because the validation process did not reject the result, it is considered a valid number. However, there were no other positive VOC results during the entire sampling period, suggesting that the toluene result may be anomalous. Without follow-up or confirmatory analyses, theories for this occurrence may include laboratory cross-contamination, field-contamination, laboratory error, or analytic anomaly. Additional sampling and analysis are recommended.

Other VOC results were reported as estimated quantities or method quantitation limits for methylene chloride, acetone, carbon disulfide, carbon tetrachloride, and trichloroethene. In summary, no volatile organic compounds are present in the aquifer beneath the new facility. 


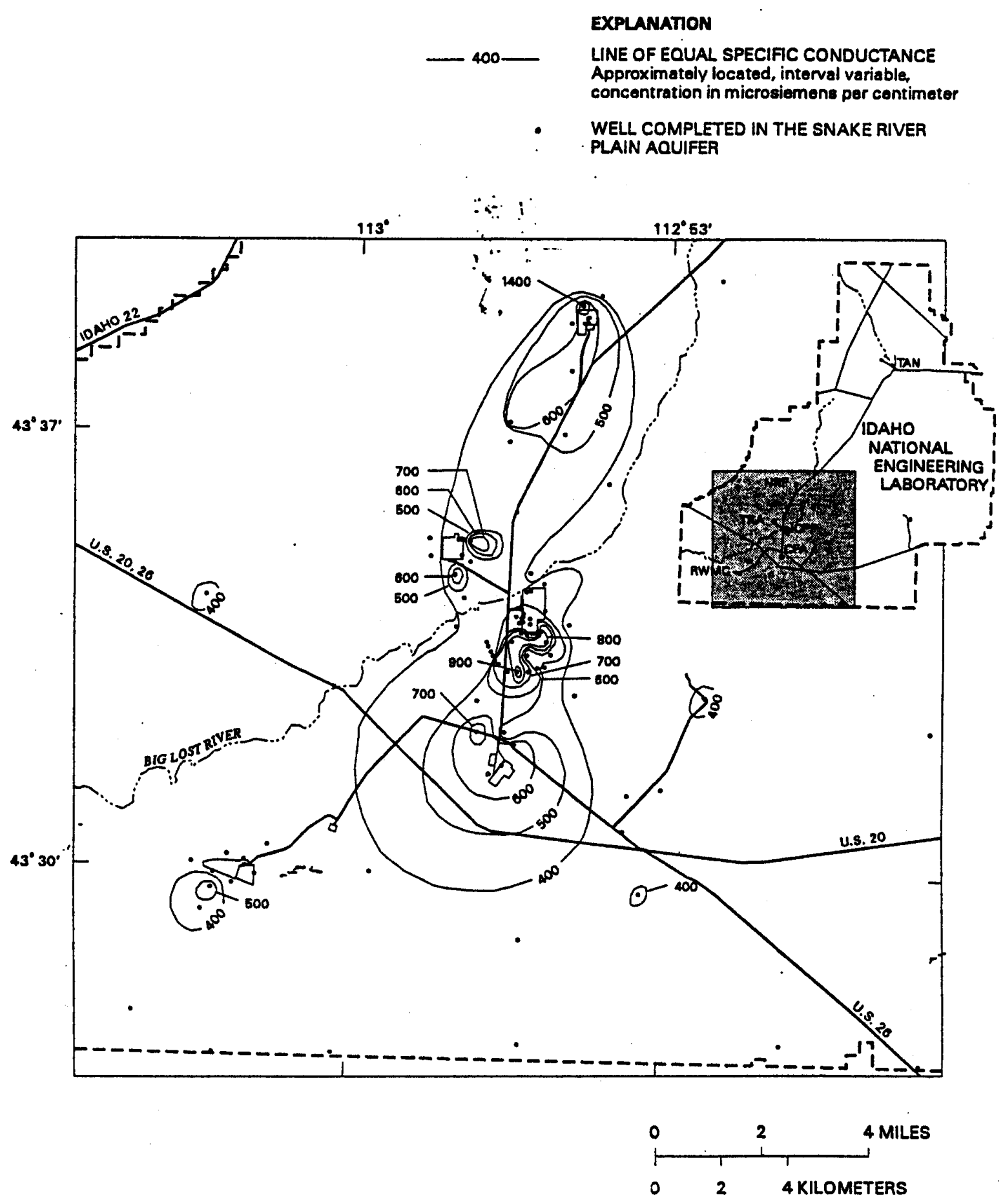

Figure 4-1. Distribution of specific conductance of water from the SRPA at the INEEL, October 1995. 


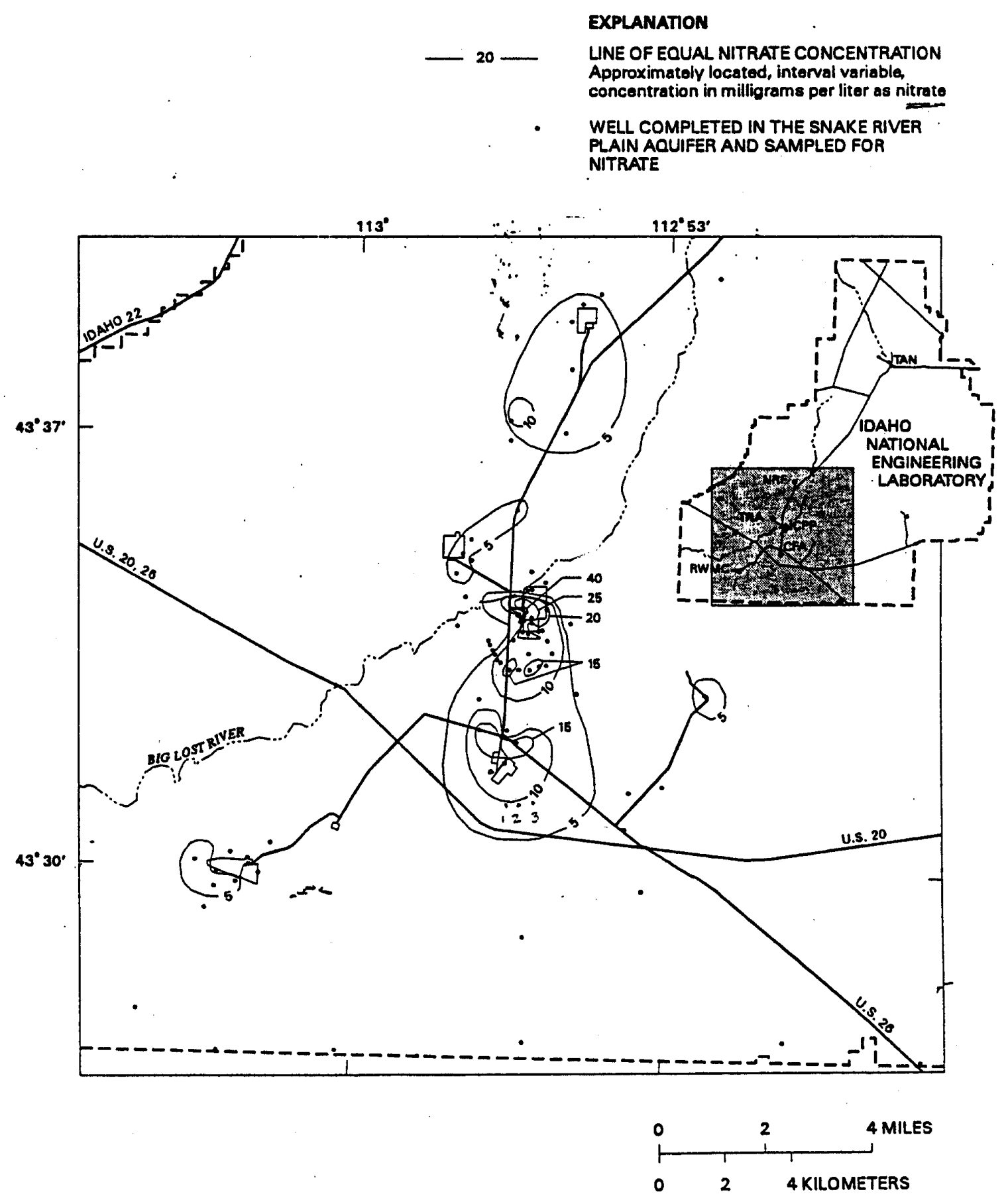

Figure 4-2. Distribution of nitrate in water from the SRPA at the INEEL, October 1995. 


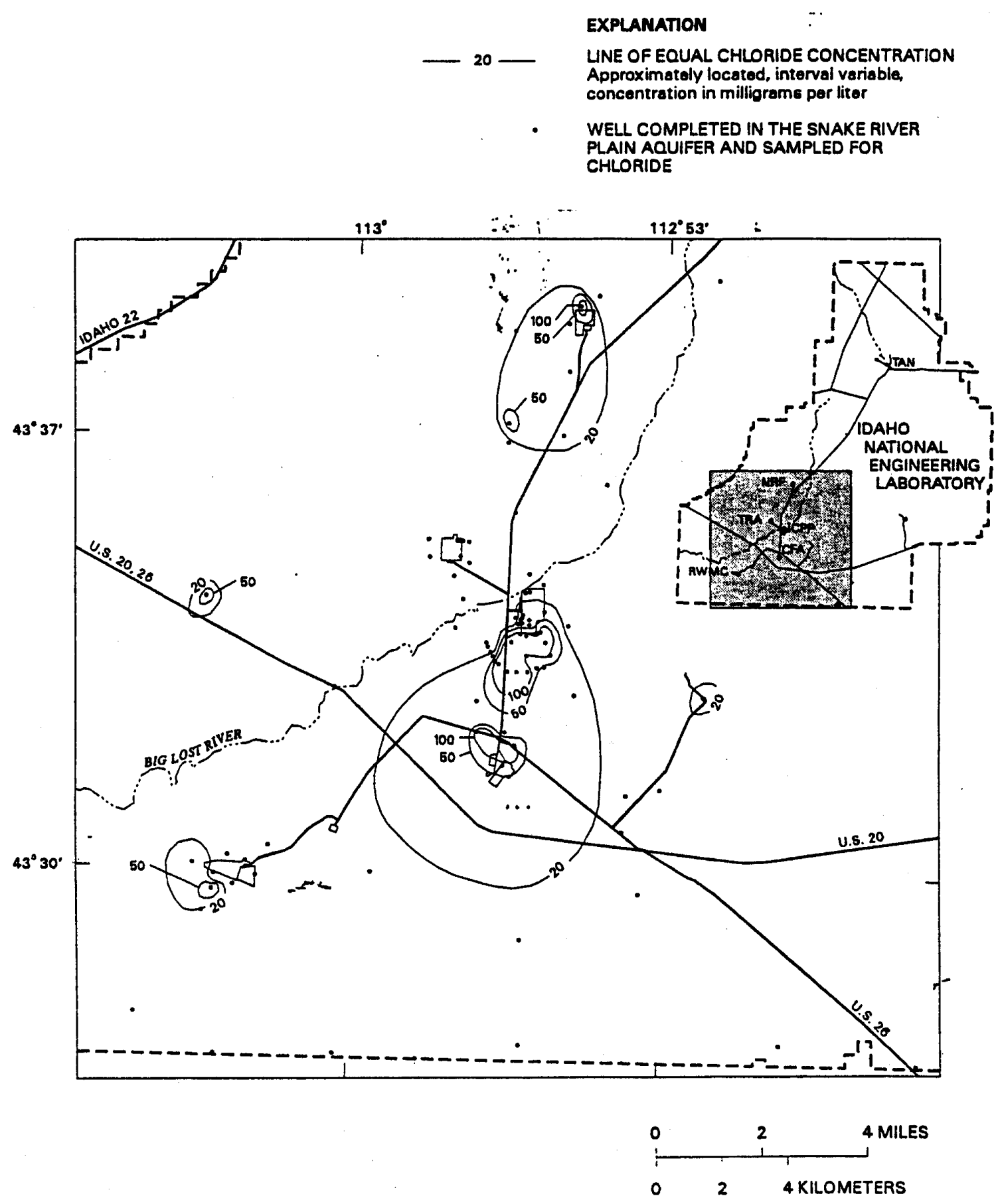

Figure 4-3. Distribution of chloride in water from the SRPA at the INEEL, October 1995. 


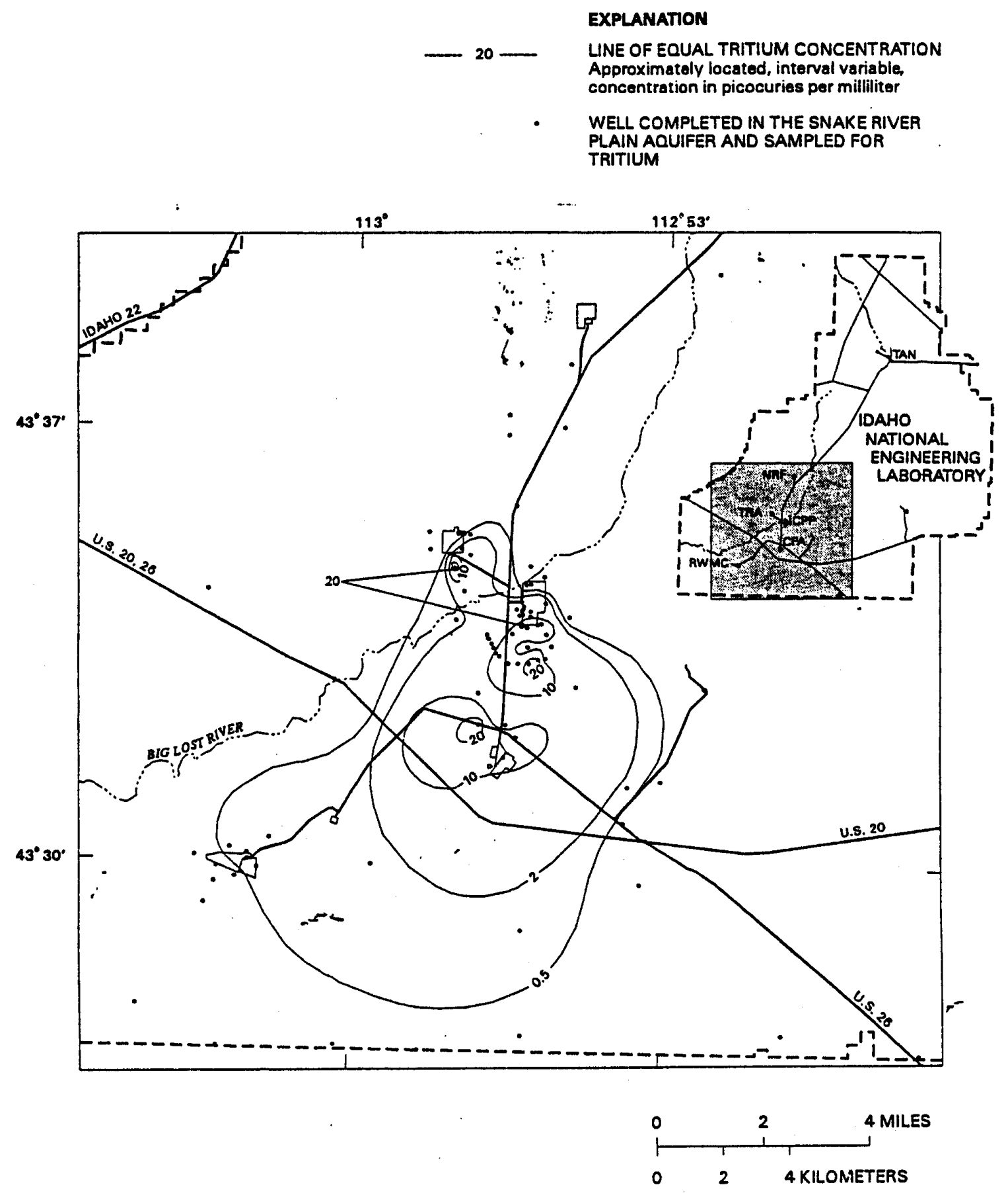

Figure 4-4. Distribution of tritium in water from the SRPA at the INEEL, October 1995. 


\section{EXPLANATION}

8 LINE OF EQUAL STRONTIUM-90 CONCENTRATION

Approximatoly locatod, interval voriable.

concentration in picocuries per liter

WELL COMPLETED IN THE SNAKE RIVER

PLAIN AQUIFER AND SAMPLED FOR

STONTIUM-90

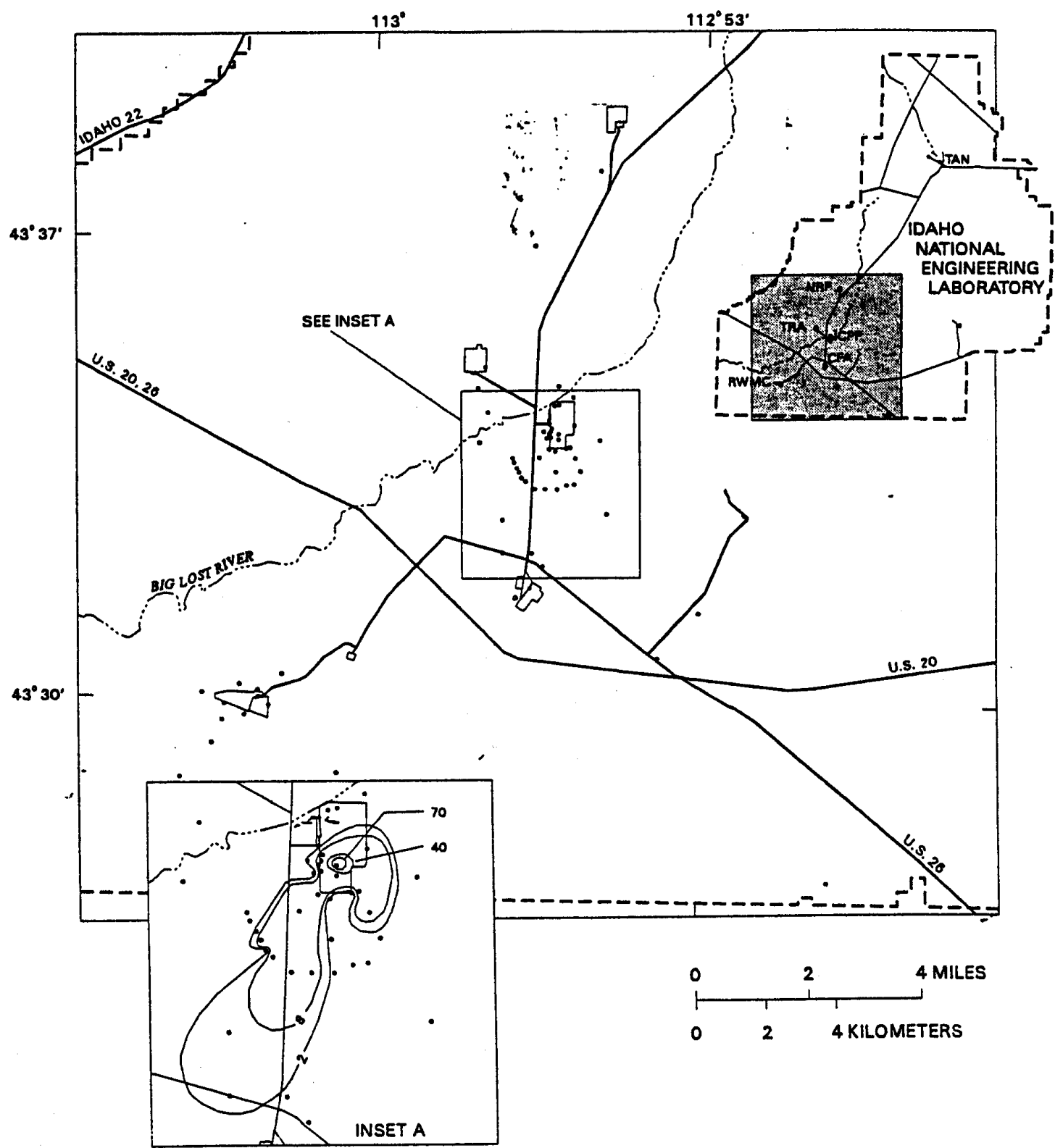

Figure 4-5. Distribution of strontium-90 in water from the SRPA at the INEEL, October 1995. 


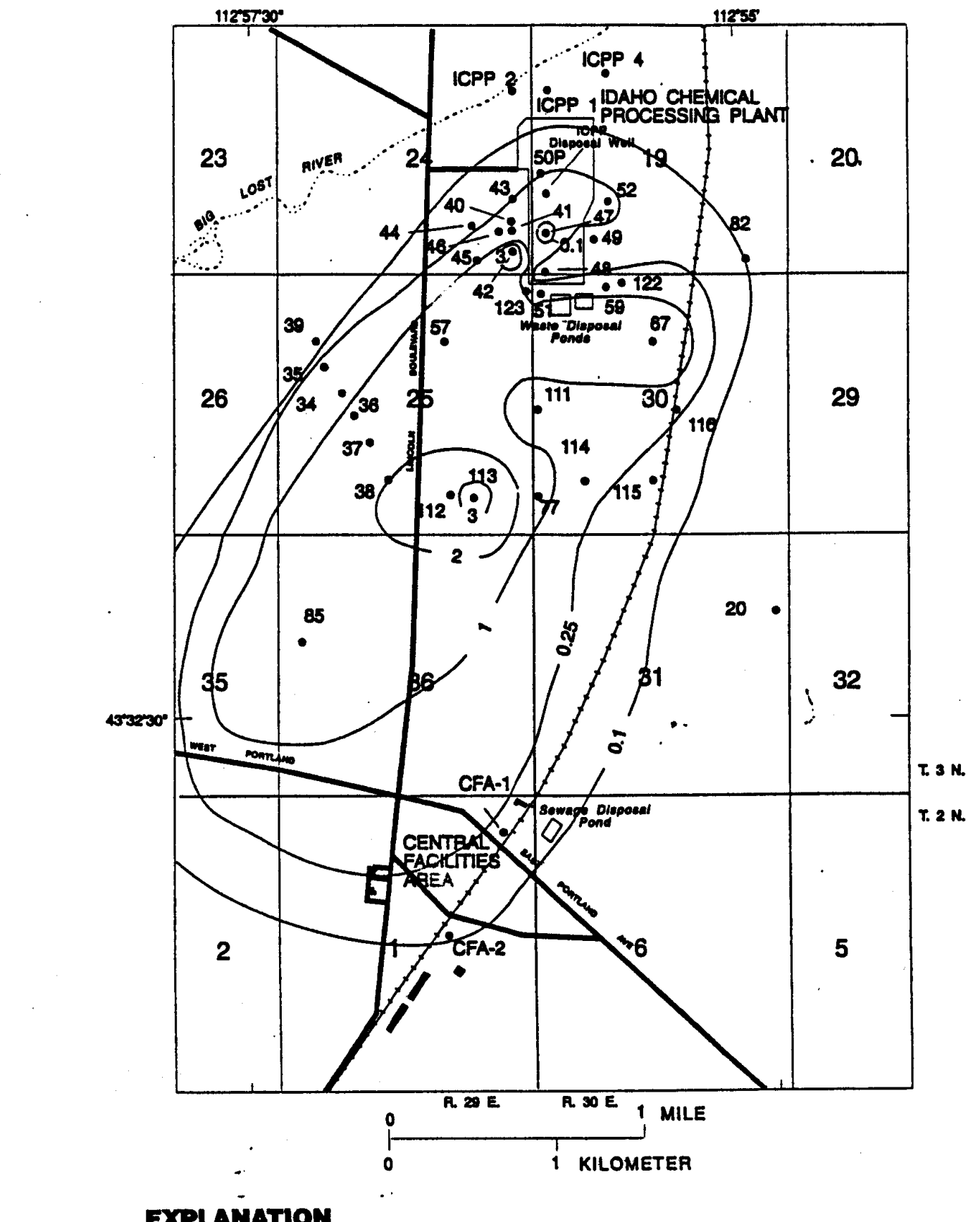

-1 - LINE OF EQUAL IODINE-129 CONCENTRATION--Interval, in picocuries per liter, is variable

57 - WELL FROM WHICH WATER SAMPLE FOR IODINE-129 WAS COLLECTED IN 1990-91-Number, 57, is local well identifier, $P$ indicates well obtains water trom a perched ground-water zone. See table 2 for iodine-129 concentrations in specific wells

Figure 4-6. Areal distribution of iodine-129 in the SRPA near the ICPP, 1990-91. 
Analyses for inorganic compounds also show that no constituents are present above their respective MCLs in groundwater beneath the new facility. The highest measurement for total dissolved solids was $249 \mathrm{mg} / \mathrm{L}$ (MCL $500 \mathrm{mg} / \mathrm{L}$ ). The highest measurement for chloride, although not analyzed during each round in all wells, was $10.9 \mathrm{mg} / \mathrm{L}$ (MCL $250 \mathrm{mg} / \mathrm{L}$ ).

Metals analyses show that results for most constituents were below their respective MCLs. In well ICPP-MON-A-166, March and April 2001 data show that levels of aluminum, manganese, and iron were above the secondary maximum contaminant levels (SMCL). In well ICPP-MON-A-167, levels of aluminum, manganese, and iron exceeded the SMCL in all months. Although the exact source of these detections is unknown, they could be attributed to a combination of natural causes (basalt chemistry) and well construction materials.

Radiological results, when compared to derived concentration guides (DCGs) published in DOE Order 5400.5, show that all isotopic analyses for gamma and alpha emitters were below their respective DCGs. For radiological constituents having a specified MCL, results for beta/photon emitters, strontium-90, and tritium were below their respective MCL. The maximum result for beta/photon emitters was $9.8 \mathrm{pCi} / \mathrm{L}$, which is consistent with concentrations observed in groundwater beneath the INTEC facility, in the service wastestream, and in the raw water supply. The November 2000 and February 2001 gross alpha analyses from ICPP-MON-A-167 may indicate exceedances of the MCL (5 pCi/L). With each of these reported values there is an associated uncertainty. If the uncertainties are taken in to account, the analytical results either drop below or exceed the MCL. Additionally, regulations state that if the gross alpha MCL is exceeded, one must also determine whether Ra-226 and Ra-228, added together, exceed $5 \mathrm{pCi} / \mathrm{L}$. Because this necessary information is not available, one cannot determine, with certainty, if there is a legitimate exceedance of the gross alpha MCL. Additional sampling and analyses are recommended.

\subsection{Perched Water Monitoring and Sampling}

All vadose zone wells and boreholes within the vicinity of the new facility were either dry or unable to yield enough water during the sampling period; therefore, no water samples were collected. Wells installed provide the capability of monitoring perched water occurring at the following depths: 34 - $59 \mathrm{ft}$ bls and 113 - $131 \mathrm{ft}$ bls. During drilling activities, perched water was observed at the following depths: 170, 198, 235, and $299 \mathrm{ft}$ bls.

\subsection{Sample Analysis and Data Validation}

All data are reported as Tier 1 and received Level A validation. All data are entered in the ERIS database and hard copies of data and limitations and validation reports are housed in project management files and the INEEL Hydrologic Data Repository. All documentation relative to data collection and analysis is listed in Section 6, References.

\subsection{Groundwater Isotope Sampling}

Additional groundwater samples were collected from ICPP-MON-A-164C, ICPP-MON-A-165, and ICPP-MON-A-167 and submitted for radiogenic isotopic analysis as part of an ongoing study to characterize the geologic factors that control Snake River Plain Aquifer flow, thickness, and chemistry. Analysis of these samples, representing the last opportunity to collect water from this area prior to the operation of the infiltration ponds, will further understanding of the nature of the aquifer at this part of the INEEL. Although samples have been sent for analyses, results are not expected until April 2002. These data will help interpret and identify preferential flow paths in the Snake River Plain Aquifer system, which is extremely important for understanding contaminant transport. Measurement of radiogenic isotope ratios, specifically ${ }^{87} \mathrm{Sr} /{ }^{86} \mathrm{Sr}$, in chemically evolving waters provides a highly effective preferential 
flow path indicator or tracer. Slow-flowing zones are identified as lower ${ }^{87} \mathrm{Sr} /{ }^{86} \mathrm{Sr}$ ratio areas created by prolonged contact with the host basalts. Faster flowing zones, correspondingly, have higher ${ }^{87} \mathrm{Sr} /{ }^{86} \mathrm{Sr}$ ratios. Additionally, ${ }^{234} \mathrm{U} /{ }^{238} \mathrm{U}$ ratios are effective groundwater tracers. Commonly, elemental analyses are performed in conjunction with the isotopic analyses ( $\mathrm{Li}, \mathrm{B}, \mathrm{Na}, \mathrm{Mg}, \mathrm{Al}, \mathrm{Si}, \mathrm{K}, \mathrm{Ca}, \mathrm{Mn}, \mathrm{Fe}, \mathrm{Rb}, \mathrm{Sr}, \mathrm{Ba}$, and $\mathrm{U})$. Compared to elemental concentration data, however, radiogenic isotopes may be more easily interpreted because the effects of water-rock interaction, and the equilibria toward which the isotope ratios evolve, are more predictable (Johnson et al. 2000). 


\section{ENVIRONMENTAL PROTECTION}

Groundwater beneath the INEEL is protected under the State of Idaho Groundwater Quality Requirements, Title 1, Chapter 2, "Idaho Water Quality Standards and Wastewater Treatment Requirements" (IDAPA 58.01.02); Title 1, Chapter 17, "Idaho WLAP Regulation" (IDAPA 58.01.17); Idaho Ground Water Quality Rule (IDAPA 58.01.11); DOE Order 5400.5; and other state and federal requirements. The SRPA is an extremely important fresh water resource in Idaho that yields about $8.0 \times 10^{9} \mathrm{~m}^{3}$ (6.5 million acre- $\mathrm{ft}$ ) of high quality water annually for irrigation, municipal, and industrial uses. In 1991, the aquifer was designated as a sole-source aquifer for the region.

It is the goal of the regulations to maintain "no impact" to groundwater from INEEL activities, including discharges associated with the INTEC SWDF.

\subsection{Environmental Checklist}

On October 6, 1999, the Environmental Checklist for the INTEC Percolation Pond Replacement Project (Appendix E) was approved by the INEEL National Environmental Policy Act (NEPA)/Environmental Program staff, the DOE Environmental Restoration Program Office, and the DOE NEPA Compliance Office. Potential impacts to air, surface and groundwater, ecological and archaeological resources from construction and operation activities were evaluated. Requirements for operation of the pond include fugitive dust control and a Wastewater Land Application Permit.

\subsection{Wastewater Land Application Permitting}

The State of Idaho guidelines for permitting land application of wastewater are issued under the IDAPA 58.01.17.300.04 and .05. "The Handbook for Land Application of Municipal and Industrial Wastewater" (State of Idaho 1996) also provides information and guidance for permit preparation. Permits are submitted to the State of Idaho Department of Environmental Quality (IDEQ). The new INTEC percolation ponds were designed and located to meet all the requirements of the wastewater land application regulations. Additional applicable requirements may be found in the EPA Process Design Manual for Land Treatment of Municipal Wastewater (EPA 1981) and the associated supplement Supplement on Rapid Infiltration and Overland Flow (EPA 1984).

The WLAP permit for the existing discharge to percolation ponds at the south end of INTEC was extended until December 2003. This permit requires that samples collected from process wastewater prior to discharge to the pond be analyzed for Maximum Allowable Concentration (MAC) Secondary Quality Standards except TDS at $800 \mathrm{mg} / \mathrm{L}$, chloride at $350 \mathrm{mg} / \mathrm{L}$, and excluding radiological parameters and total phosphorous.

On September 10, 2001, wastewater land application permit number LA-000130-03 was issued from the State of Idaho to DOE-ID and BBWI for operation of the INTEC SWDF. The permit expires on October 1, 2006. Compliance monitoring of groundwater was specified for two downgradient aquifer wells and for two perched water wells located immediately adjacent to the facility. The permit requires that samples collected from process wastewater prior to discharge to the pond and all groundwater monitoring points be analyzed for $\mathrm{pH}$, total Kjeldahl nitrogen, nitrate-nitrogen, nitrite-nitrogen, total phosphorous, total dissolved solids, chloride, fluoride, aluminum, arsenic, cadmium, chromium, copper, iron, manganese, mercury, selenium, silver, sodium, gross alpha particle activity (including radium-226, but excluding radon and uranium), combined beta/photon emitters, combined radium 226 and 228, strontium-90, tritium, and iodine-129. Presently, permit number LA-000130-03 is undergoing administrative appeal by DOE-ID (initiated by petition dated 11/8/01) regarding the regulation and monitoring of radionuclides in the service waste stream. 


\subsection{Wellhead Protection and Capture Zones}

An INEEL Wellhead Protection Program transmittal, dated October 16, 1997 (Appendix D), was prepared with the intent of minimizing impact to existing and future operations while establishing a program that improves groundwater protection in cases where a significant risk to INEEL water sources now exists or may exist in the future. Proposed in the document are wellhead protection zones intended to alert INEEL operations and projects personnel to the risks of groundwater and wellhead contamination in certain areas and force the implementation of appropriate controls and policies to ensure that the potential risks are evaluated prior to construction or similar activities. It is not the intent to discontinue or prohibit common INEEL activities within the Wellhead Protection Areas.

Attachment A of the above-referenced transmittal describes the requirements of the DEQ's Plan, discusses the development of the current wellhead protection zones, and addresses future program implementation tasks. Funding for the plan was terminated prior to final acceptance of the document.

Figure 5-1 illustrates the recommended wellhead protection zones for production wells CPP-01, CPP-02, and the Rifle Range Well southwest of the new facility. As shown, the location of the discharge facility lies within the six-year capture zone for the Rifle Range Well.

\subsection{Storm Water}

The INEEL must comply with the EPA Administered Permit Programs: (1) National Pollutant Discharge Elimination System (40 Code of Federal Regulations [CFR] 122), "Final National Pollutant Discharge Elimination System Storm Water MultiSector General Permit for Industrial Activities" (EPA 1995) issued by the EPA, and (2) modifications issued on September 30, 1998 (EPA 1998). [Note: On October 30, 2000, the EPA reissued the NPDES Storm Water Multi-Sector General Permit for Industrial Activities. Under this issuance, the INEEL submitted a request for coverage on January 29, 2001.] EPA's recommended approach to storm water management is through the use of storm water pollution prevention plans designed to prevent or minimize the pollution of storm water. As storm water flows over surfaces where industrial or construction activities are taking place, there is the potential for contaminants to be picked up by the water and transported to a receiving stream, which then flows to the Big Lost River. Requirements of the General Permit are applied to activities within the area of potential storm water drainage to the Big Lost River. Figure 5-2 shows an approximate area, containing the new facility, where storm water has a reasonable potential to drain to the Big Lost River System (Bennett 1990).

The Storm Water Program will manage activities that fall within the regulatory definition of storm water discharge associated with industrial activity. Examples of activities associated with the new facility that fall under the regulatory definition of an industrial activity include operation of the ponds after construction, geotechnical investigations with min imal disturbance, archaeological investigations with minimal disturbance, and borrow source operation. Industrial activities are addressed in the INEEL Storm Water Pollution Prevention Plan for Industrial Activities (SWPPP-IA) (DOE-ID 2001). 


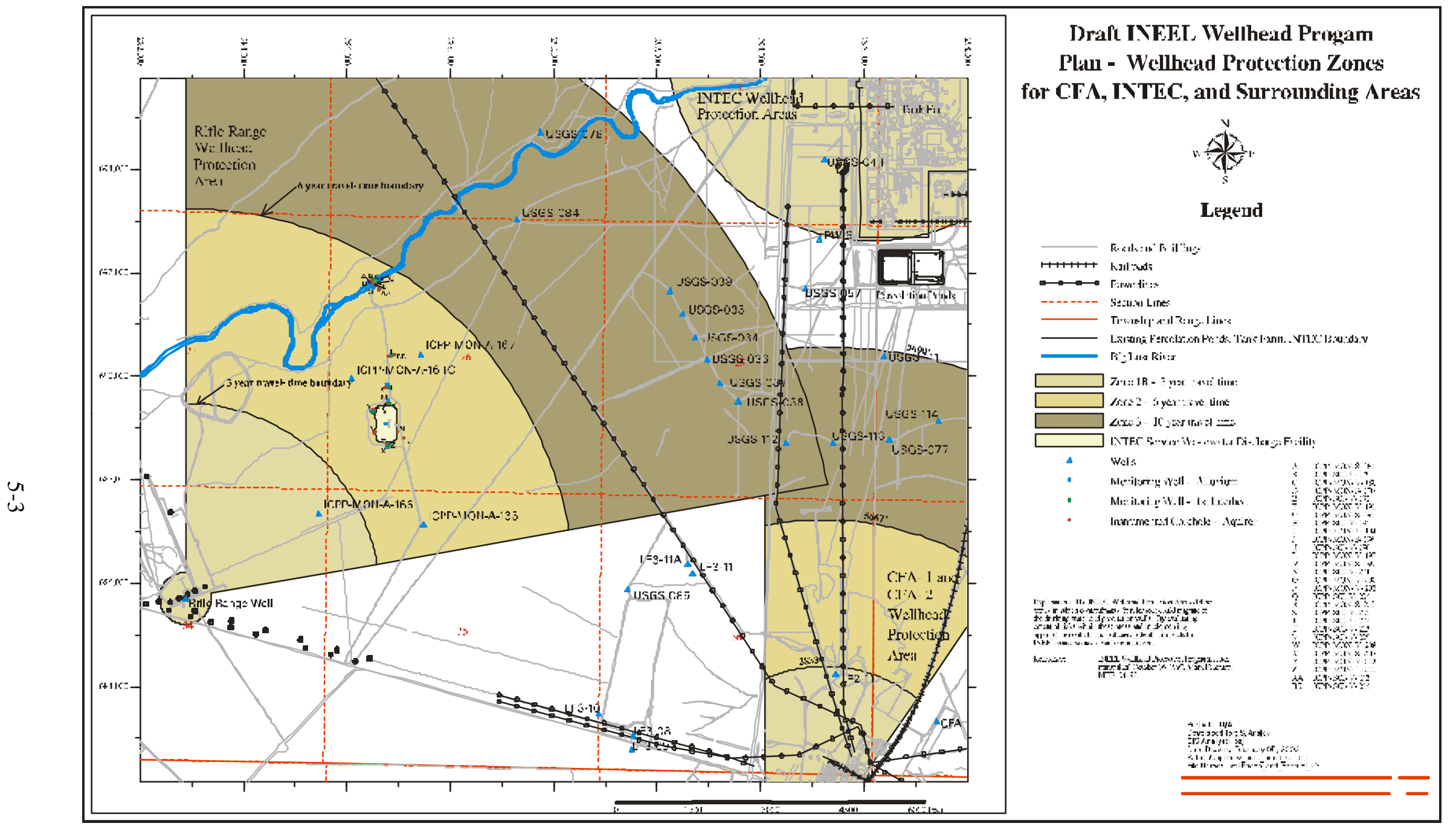

Figure 5-1. Wellhead protection zones for CFA and INTEC potable water wells. 


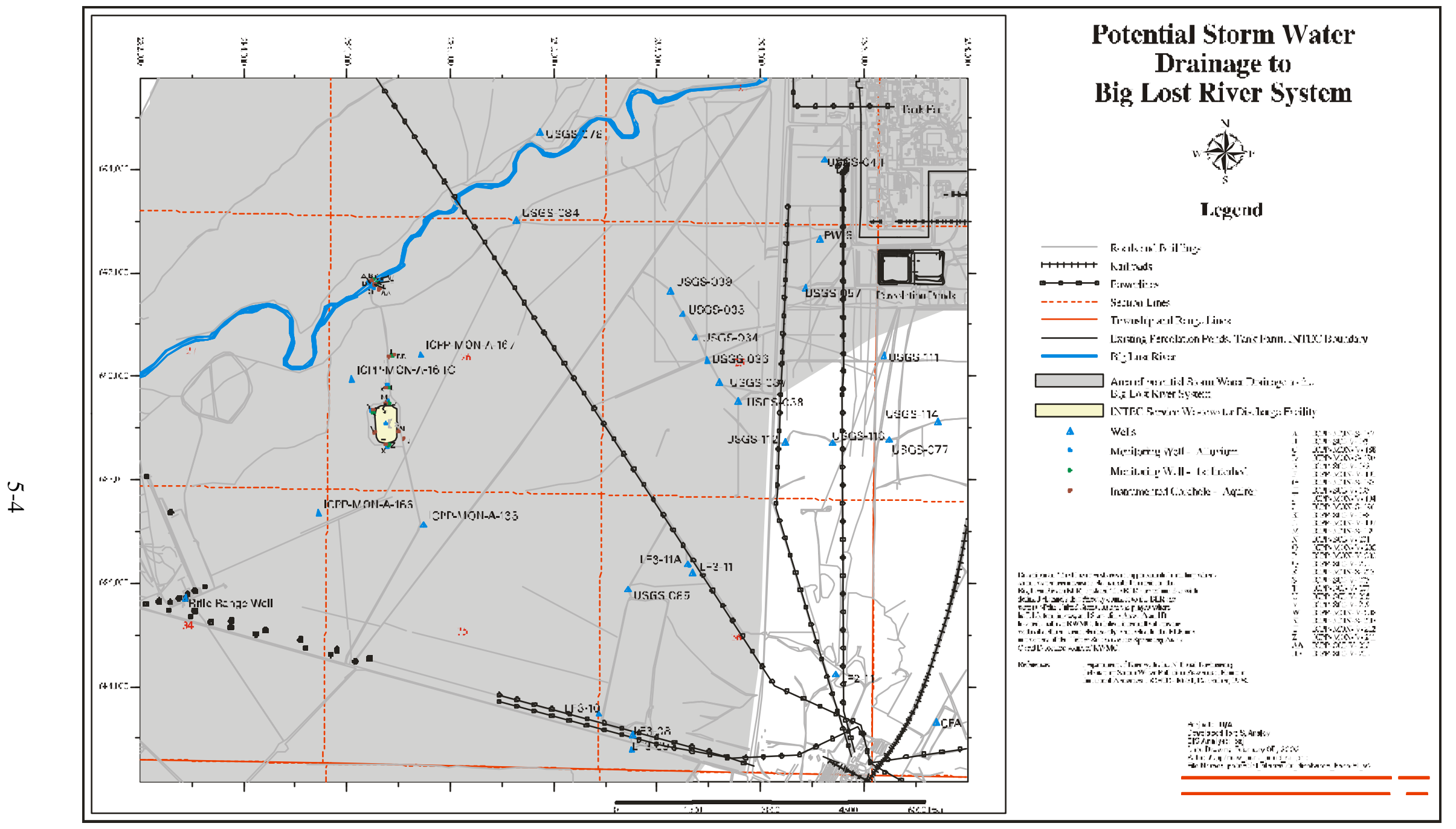

Figure 5-2. Big Lost River potential storm water drainage areas. 


\subsection{DOE Order 5400.1 Requirements}

The DOE Order 5400.1, “General Environmental Protection Program,” establishes environmental program requirements, authorities, and responsibilities for Department of Energy operations for assuring compliance with applicable federal, state, and local environmental protection laws and regulations, executive orders, and internal department policies. Therefore, it is DOE policy to ensure all operations are conducted within compliance and according to good environmental management practices. Additionally, DOE is committed to correct existing environmental problems, to minimize risks to the environment or public health, and anticipate and address potential environmental problems before they pose a threat to the quality of the environment or the public welfare.

Chapter IV, Section 3, of DOE Order 5400.1 requires that an environmental study shall be conducted prior to start up of a new site, facility, or process that has the potential for significant adverse environmental impact. The preoperational study should begin not less that 1 year, and preferably 2 years before start up to evaluate seasonal changes. The study shall serve to: characterize existing physical, chemical, and biological conditions that could be affected; establish background levels of radioactive and chemical components; characterize pertinent environmental and ecologic parameters; and identify potential pathways for human exposure or environmental impact as a basis for determining the nature and extent of the subsequent routine operational and emergency effluent monitoring and environmental surveillance programs. Where time and circumstances do not allow for completion of preoperational monitoring prior to start-up, it shall be conducted concurrent with work on the new site, facility, or process. The preoperational study shall be consistent with NEPA compliance activities. Where appropriate, activities and documentation conducted for NEPA compliance may substitute for compliance with this requirement. [Note: Although NEPA is not performed for CERCLA activities, CERCLA performs an "equivalent" evaluation through the CERCLA process.]

Pertinent to the INTEC SWDF, effluent monitoring must be able to: 1) verify compliance with applicable federal, state, and local effluent regulations and DOE orders; 2) determine compliance with commitments made in an EIS, EA, or other official documentation; 3) evaluate the effectiveness of effluent treatment and control; 4) identify potential environmental problems and evaluate the need for remedial actions or mitigation measures; 5) support permit revision and/or reissuance; and 6) detect, characterize, and report unplanned releases. All monitoring data shall be maintained as auditable records in accordance with the requirements of DOE O 414.1A. Additionally, environmental surveillance activities, specifically ambient groundwater and effluent water quality monitoring shall be conducted to: 1) characterize and define trends in the physical, chemical, and biological condition of environmental media; 2) establish baselines of environmental quality; 3) provide a continuing assessment of water pollution control programs; 4) identify new water quality problems; and 5) detect, characterize, and report unplanned releases and their effects on water quality. Ambient water quality monitoring serves to confirm compliance with the Clean Water Act (33 USC 1251 et seq.). Specific ally, groundwater quality monitoring, in addition to the above information, will: 1) provide data to permit the early detection of groundwater pollution or contamination; 2) provide a reporting mechanism for detected groundwater pollution or contamination; 3) provide data upon which decisions can be made concerning land disposal practices and the management and protection of groundwater resources; and 4) identify existing and potential groundwater contamination sources and to maintain surveillance of these sources. 


\section{REFERENCES}

40 CFR 122, 2000, "EPA Administered Permit Programs: The National Pollutant Discharge Elimination System," Code of Federal Regulations, Office of the Federal Register, July 1, 2000.

33 USC 1251 et seq., 1977, "Clean Water Act," as amended.

Akerman, D. J., 1991, Transmissivity of the Snake River Plain Aquifer at the Idaho National Engineering Laboratory, Idaho, Water Resources Investigation Report 91-4058, U.S. Geological Survey for the U.S. Department of Energy Idaho Operations Office, DOE/ID-22097, 1991.

Ansley, Shannon to Lee Davison, December 10, 1998, "Evaluation of Historical Service Waste Sample Data for INTEC Percolation Ponds - SLA-02-98."

Bennett, C. M., 1986, Capacity of the Diversion Channel Below the Flood-Control Dam on the Big Lost River at the Idaho National Engineering Laboratory, U.S. Geological Survey, Water Resources Investigations Report 86-4204, 1986.

Bennett, C. M., 1990, Stream-flow Losses and Ground-water Level Changes Along the Big Lost River at the Idaho National Engineering Laboratory, Idaho, U.S. Geological Survey, Water Resources Investigation Report 90-4067, April 1990.

Berenbrock, C., and L. C. Kjelstrom, 1997, Simulation of Water-Surface Elevations and Flood Boundaries for a Hypothetical 100-Year Peak Flow in the Big Lost River and at the Idaho National Engineering and Environmental Laboratory, Idaho, U.S. Geological Survey, Water Resources Investigations Report, 1997.

Berenbrock, C. and L. C. Kjelstrom, 1998, Preliminary Water-Surface Elevations and Boundary of the 100-Year Peak Flow in the Big Lost River at the Idaho National Engineering and Environmental Laboratory, Idaho, U.S. Geological Survey, Water Resources Investigations Report 98-4065, DOE/ID-22148, 1998.

DEQ, 1997, Idaho Wellhead Protection Plan, Idaho Division of Environmental Quality, February.

DOE O 414.1A, 1999, “Quality Assurance,” U.S. Department of Energy, September 29, 1999.

DOE O 5400.1, 1990, “General Environmental Program,” U.S. Department of Energy, June 29, 1990.

DOE O 5400.5, 1993. "Radiation Protection of the Public and the Environment," U.S. Department of Energy, Washington, D.C., January 7, 1993.

DOE-ID, 1997, Comprehensive RI/FS for the Idaho Chemical Processing Plant WAG 3 OU 3-13 at the INEEL—Part A RI/BRA Report (Final), U.S. Department of Energy, Idaho Operations Office, DOE/ID-10534, November, 1997.

DOE-ID, 1999a, Final Record of Decision, Idaho Nuclear Technology and Engineering Center, Operable Unit 3-13, DOE/ID-10660, Rev. 0, October 1999.

DOE-ID, 1999b, Evaluation and Site Selection for a New Service Waste Disposal Facility for the Idaho Nuclear Technology and Engineering Center, DOE/ID-10705, Revision 0, September 1999. 
DOE-ID, 2000, Interim Control Strategy for the INTEC Service Wastewater Discharge Facility

(Percolation Pond Replacement), DOE/ID-10824, Revision 0, December 2000.

DOE-ID, 2001, INEEL Storm Water Pollution Prevention Plan for Industrial Activities, SWPPP-IA, DOE/ID-10431, January 2001.

EDF-2602, 2001, "Evaluate Service Waste Sources for Radiological Discharge Risk," Revision 0, April 16, 2001.

EDF-ER-249, "History of INTEC Service Wastewater Characterization Activities," INEEL/EXT-2000-01436, Revision A, undated.

EPA, 1981, Process Design Manual for Land Treatment of Municipal Wastewater, U.S. Environmental Protection Agency, October 1981.

EPA, 1984, Supplement on Rapid Infiltration and Overland Flow, U.S. Environmental Protection Agency, EPA 625/1-81-031a, October 1984.

EPA, 1995, "Final National Pollutant Discharge Elimination System Storm Water Multi-Sector General Permit for Industrial Activities," Federal Register, U.S. Environmental Protection Agency, Vol. 60, No. 189, September 1995.

EPA, 1998, "Modification of the National Pollutant Discharge Elimination System (NPDES) Storm Water Multi-Sector General Permit for Industrial Activities," Federal Register, U.S. Environmental Protection Agency, Vol. 63, No. 189, September 1998.

Feldman, M., 1997, "INEEL Wellhead Protection Program,” MEF-04-97, letter report to distribution, LIMITCO, October 16, 1997.

Holdren, K. J., D. Burgess, K. N. Keck, D. L. Lowrey, M. J. Rohe, R. P. Smith, C. S. Staley, J. Banaee, 1997, Preliminary Evaluation of Potential Locations on the Idaho National Engineering and Environmental Laboratory for a High-Level Waste Treatment and Interim Storage Facility and a Low-Level Waste Landfill, Lockheed Martin Idaho Technologies Company, INEEL/EXT-97-01324, Rev. 0, December 1997.

IDAPA 58.01.02, 2000, "Water Quality Standards and Wastewater Treatment Requirements,” Idaho Administrative Procedures Act, Idaho Department of Environmental Quality, April 5, 2000.

IDAPA 58.01.11, 2001, “Ground Water Quality Rule,” Idaho Administrative Procedures Act, Idaho Department of Environmental Quality, February 2001.

IDAPA 58.01.17, 1988, "Wastewater Land Application Permit Rules," Idaho Administrative Procedures Act, Idaho Department of Environmental Quality, April 1, 1988.

INEEL, 2000a, 1999 Annual Wastewater Land Application Site Performance Reports for the Idaho National Engineering and Environmental Laboratory, Bechtel BWXT Idaho, LLC, INEEL/EXT-2000-00078, February 2000.

INEEL, 2000b, "Sampling and Analysis Plan - Pre-Operational Baseline Groundwater Monitoring for New INTEC Percolation Ponds," SAP-EM-GW-3, Revision 0, October 2000. 
INEEL, 2001, 2000 Wastewater Land Application Site Performance Reports for the Idaho National Engineering and Environmental Laboratory, INEEL/EXT-2000-01681, February 2001.

Johnson, T. M., R. C. Roback, T. L. McLing, T. D. Bullen, D. J. Depaolo, C. Doughty, R. J. Hunt, M. T. Murrell, and R. W. Smith, 2000, "Groundwater 'Fast Paths' in the Snake River Plain Aquifer: Radiogenic Isotope Ratios as Natural Groundwater Tracers,” Geology, 28, pp. 871-874.

Kjelstrom, L. C. and C. Berenbrock, 1996, Estimated 100-Year Peak Flows and Flow Volumes in the Big Lost River and Birch Creek at the Idaho National Engineering Laboratory, Idaho, U.S. Geological Survey, Water Resources Investigations Report 96-4163.

Lindholm, G. F., 1981, Plan of Study for the Regional Aquifer System Analysis of the Snake River Plain, Idaho, and Eastern Oregon, U.S. Geological Survey, Open-File Report 81-689, 1981.

LMITCO, 1995, ICPP Environmental Monitoring Report for CY-1994, Lockheed Martin Idaho Technologies Company, INEL-95/0312.

LMITCO, 1996a, ICPP Environmental Monitoring Report for CY-1995, Lockheed Martin Idaho Technologies Company, INEL-96/0186.

LMITCO, 1996b, 1995 Annual Wastewater Land Application Site Performance Reports for the Idaho National Engineering and Environmental Laboratory, Lockheed Martin Idaho Technologies Company, INEL-96/0009, January.

LMITCO, 1997a, 1996 Annual Wastewater Land Application Site Performance Reports for the Idaho National Engineering and Environmental Laboratory, Lockheed Martin Idaho Technologies Company, INEL-96/0480, January.

LMITCO, 1997b, ICPP Environmental Monitoring Report for CY-1996, Lockheed Martin Idaho Technologies Company, INEEL/EXT-97-00587.

LMITCO, 1998a, 1997 Annual Wastewater Land Application Site Performance Reports for the Idaho National Engineering and Environmental Laboratory, Lockheed Martin Idaho Technologies Company, INEEL/EXT-98-00154, February.

LMITCO, 1998b, ICPP Environmental Monitoring Report for CY-1997, Lockheed Martin Idaho Technologies Company, INEEL/EXT-98-00154.

LMITCO, 1999, 1998 Annual Wastewater Land Application Site Performance Reports for the Idaho National Engineering and Environmental Laboratory, Lockheed Martin Idaho Technologies Company, INEEL/EXT-99-00123, February.

McKinney, J. D., 1985, Big Lost River 1983-1984 Flood Threat, PPD-FPB-002, EG\&G Idaho Inc., Idaho Falls, ID.

Ostenaa, D. A., D. R. Levish, and R. E. Klinger, 1999a, "Phase 2 Paleohydrologic and Geomorphic Studies for the Assessment of Flood Risk for the Idaho National Engineering and Environmental Laboratory, Idaho," Report 99-7, Geophysics, Paleohydrology, and Seismotectonics Group, Technical Service Center, Bureau of Reclamation, Denver, CO, 1999. 
Ostenaa, D. A., D. R. Levish, R. E. Klingler, and R. A. Watts, 1999b, "Holocene Paleofloods on the Big Lost River, INEEL, Idaho (Abstract)," Geological Society of America, Rocky Mountain Section Meeting, Pocatello, Idaho, “Abstracts with Programs," 1999.

Scott, W. E., 1982, Surficial Geologic Map of the Eastern Snake River Plain and Adjacent Areas, Idaho and Wyoming, U.S. Geological Survey, Miscellaneous Investigation Map I-1372.

State of Idaho, 1996, "Handbook for Land Application of Municipal and Industrial Wastewater," April 1996.

Stone, M. A. J., L. J. Mann, and L. C. Kjelstrom, 1992, Statistical Summaries of Stream Flow Data for Selected Gaging Stations on or Near the Idaho National Engineering Laboratory, Idaho, Through September 1990, Water Resources Investigations Report 92-4196, U.S. Geological Survey for the U.S. Department of Energy Idaho Operations Office, DOE/ID-22109, 1992.

Tullis, J. A. and K. N. Koslow, 1983, Characterization of Big Lost River Floods with Recurrence Intervals Greater Than 25 Years, EG\&G Idaho, Inc., RE-PB-83-044, Idaho Falls, ID, 1983.

USACOE, 1991, Feasibility Report, Big Lost River Basin, Idaho, U.S. Army Corps of Engineers, Walla Walla, Washington, 1991.

USGS, 1997, "Hydrologic Conditions and Distribution of Selected Radiochemical and Chemical Constituents in Water, Snake River Plain Aquifer, Idaho National Engineering Laboratory, Idaho, 1992 through 1995," U.S. Geological Survey, Water-Resources Investigations Report 97-4086.

WINCO, 1994, ICPP Environmental Monitoring Report for FY-1993, Westinghouse Idaho Nuclear Company, Inc., WINCO-1207. 


\section{Appendix A}

\section{Well and Borehole Completion Reports}


A-2 
WELL NAME: ICPP-MON-V-164A Facility: Perc Ponds

Well Type: Monitoring

Well Status Active

Year Drilled: 2000

Total Depth $\frac{240}{12 / 2 / 9}$

End Date $1 / 10 / 00$

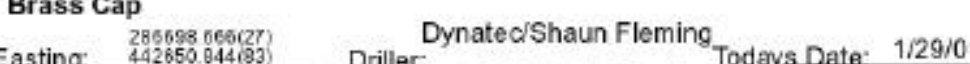

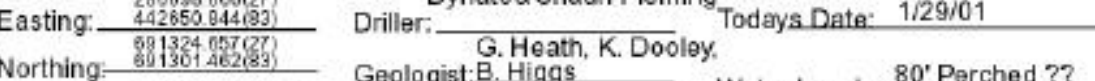

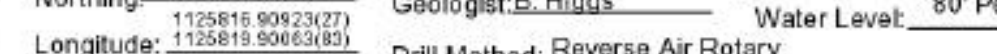

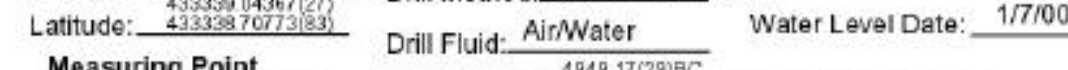

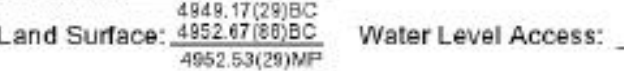

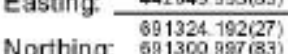

$\frac{11258.62972}{11258}$

$49335909500(27)$

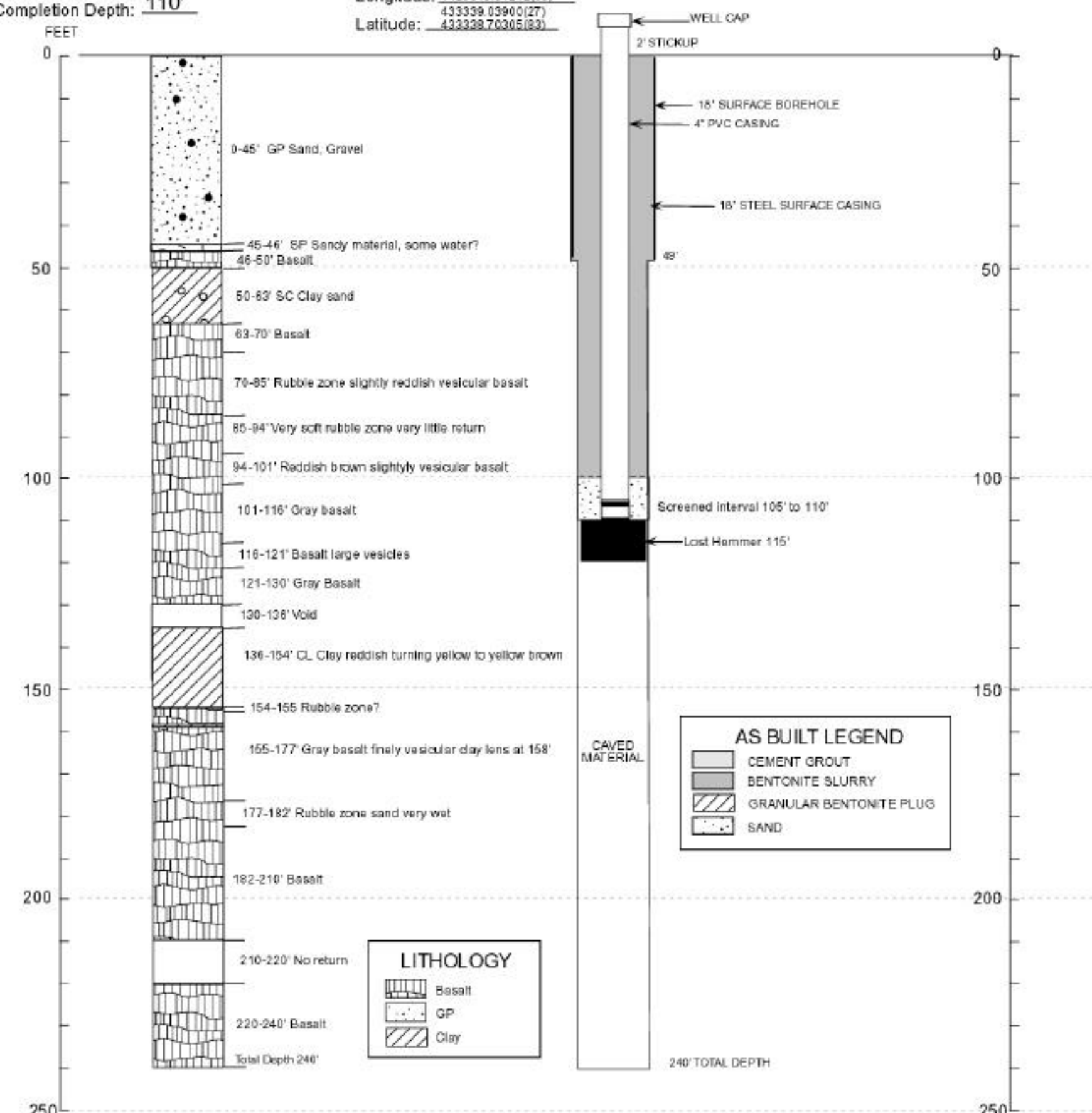

Natural Gamma

Neutron

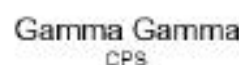

Caliper

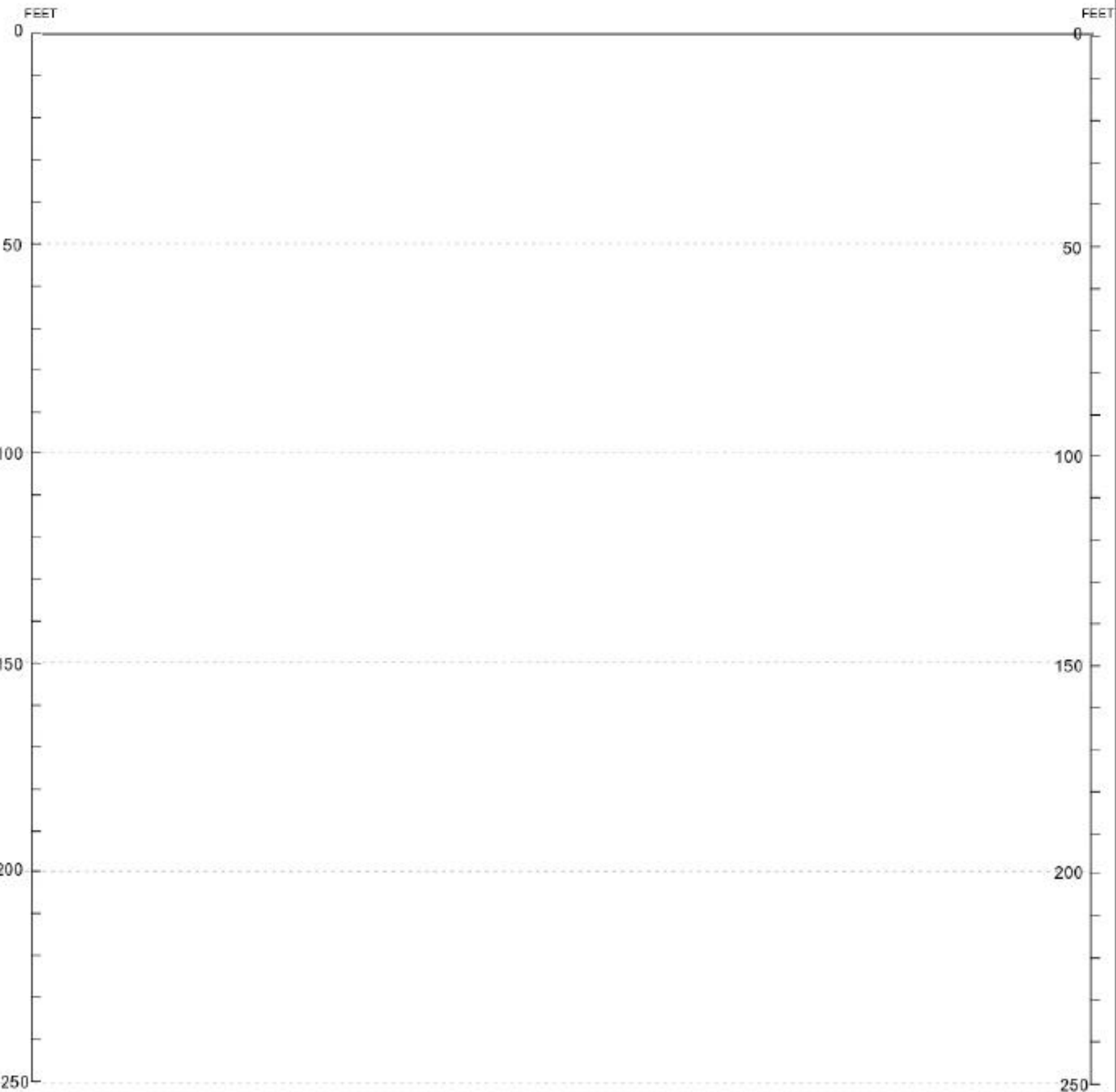


WELL NAME: ICPP-MON-A-164B Facility: Perc Ponds Well Type: Monitoring

Well Status Active

Year Driled; 2000

Total Depth $\frac{585}{1 / 1200}$

$3 / 1500$

Completion Depth. $533^{\prime}$

Completion
FEET

Brass Cap

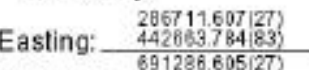

Driller:Dynateds. Fleming Todays Date: 1/29/01

Geologist:G. Heath, K. Dooley, Watr Lovel.

Drill Method: Reverse Air Rotary

Longitude: $\frac{11258919.7283}{433338.66911127}$

Lattude. 4som

uid: AirNater

Easting: 4426830404

Northing: $\frac{6912282.83963}{11258716.74127}$

Longitude: 11

(200

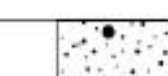

$+$.

- . 0.50 GP Sard Grove

-

i. $50-55$ ' Easalt

-

IIt

प1.1. 68 -120' Basat, aras sold

TT

1IIII)

Adish Pasalt scmen minor clary

122-137' SP Ras damp gand hiterbed,

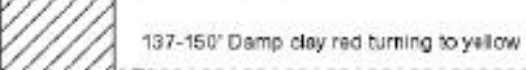

150-168: Sold Basat

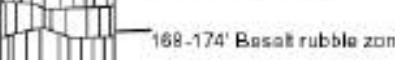

TIIIIIE-174-179. Sold Basal

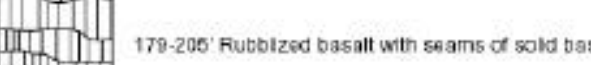

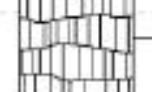

205-238: solit Baser

IIIII

IIIT-238-240' Rubble zene

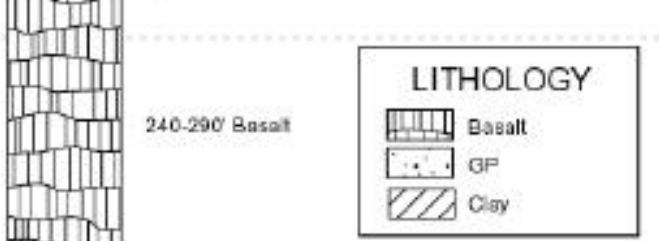

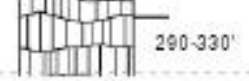

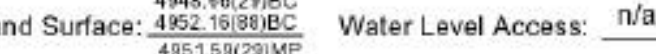
$4955.08(68) \mathrm{MP}$
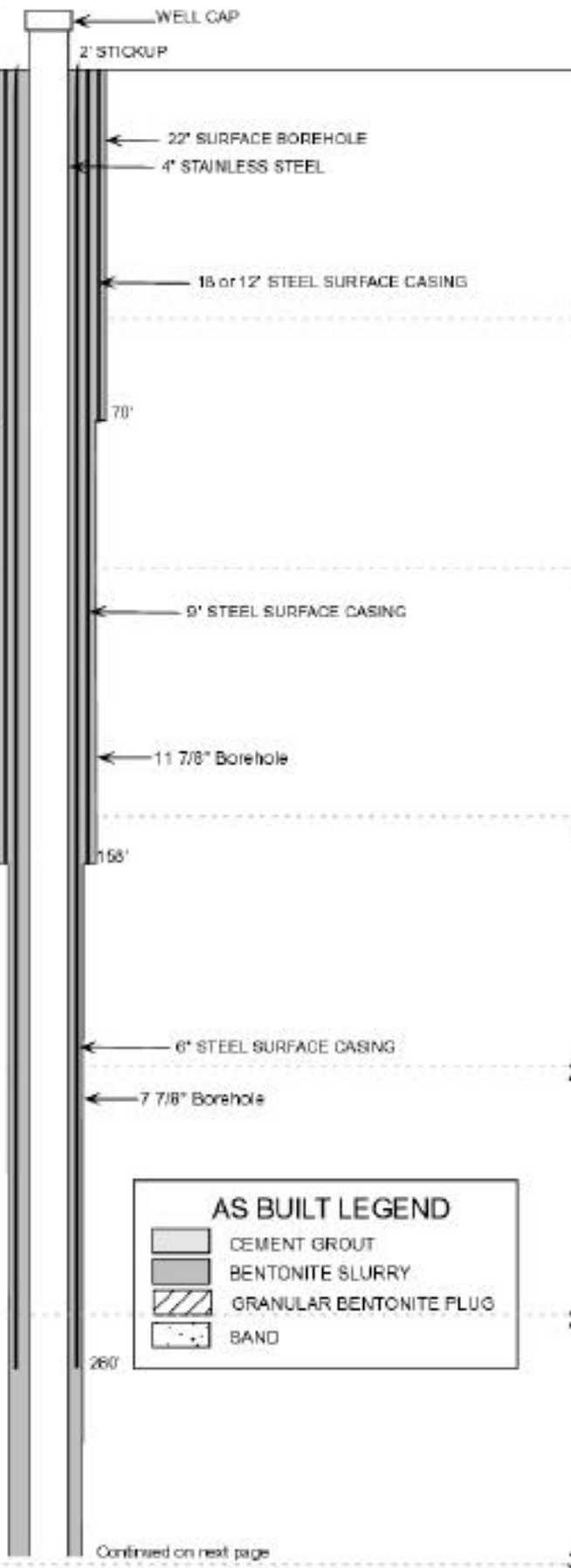

300

\section{ICPP-MON-A-164B}

Natural Gamme

Neutron

$\underset{\text { CPS }}{\text { Gamma Gamma }}$

Caliper
INCH

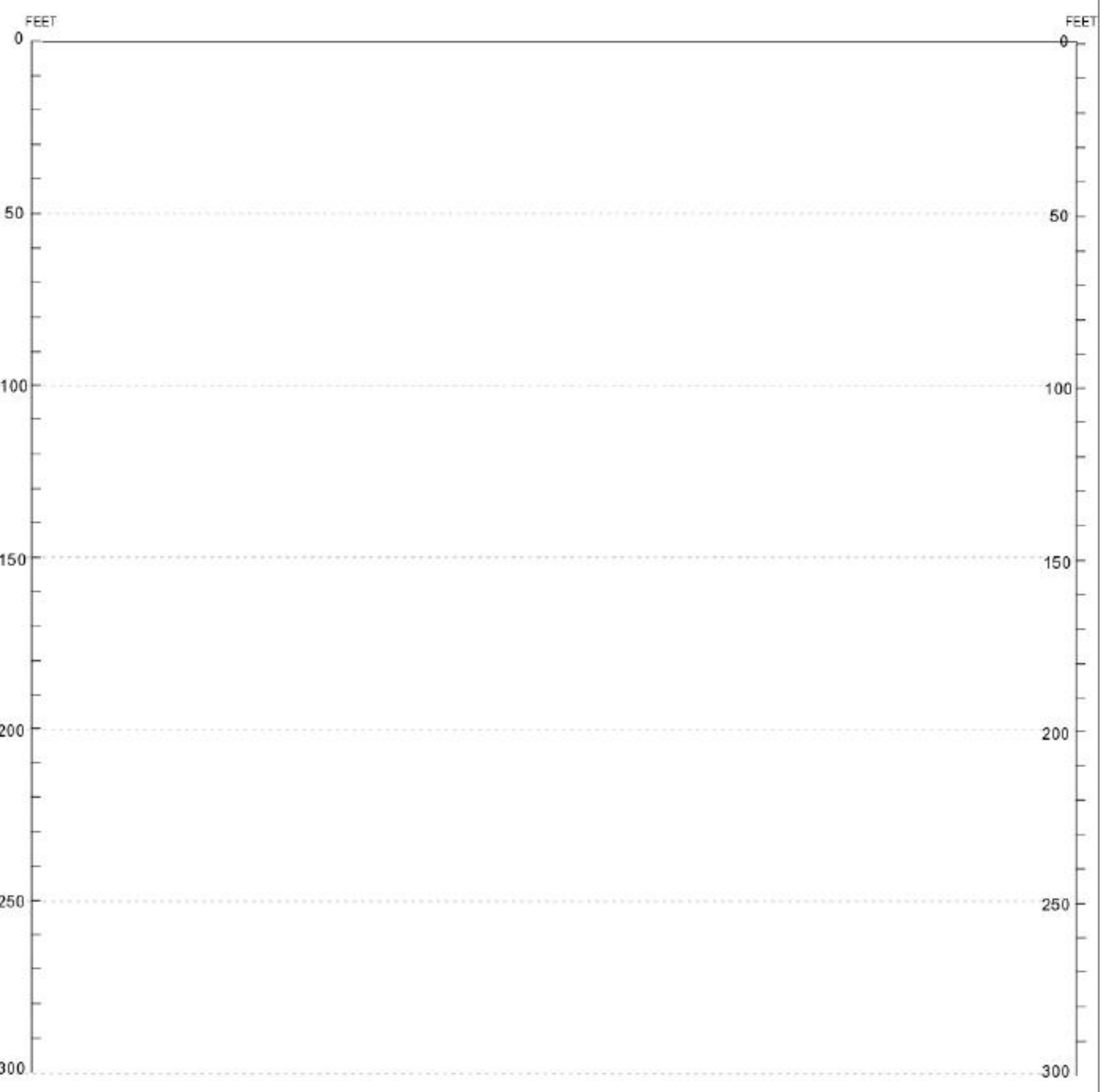


CPP-MON-A-164B

WELL NAME: page 2

Facility: Perc Ponds

Well Type: Monitoring

Well Status Active

Year Driled: 2000

Total Depth $585^{\circ}$

Start Date $\frac{1 / 12 / 00}{3 / 15 / 00}$

Complettion Depth: $\underline{533^{\prime}}$

FEET
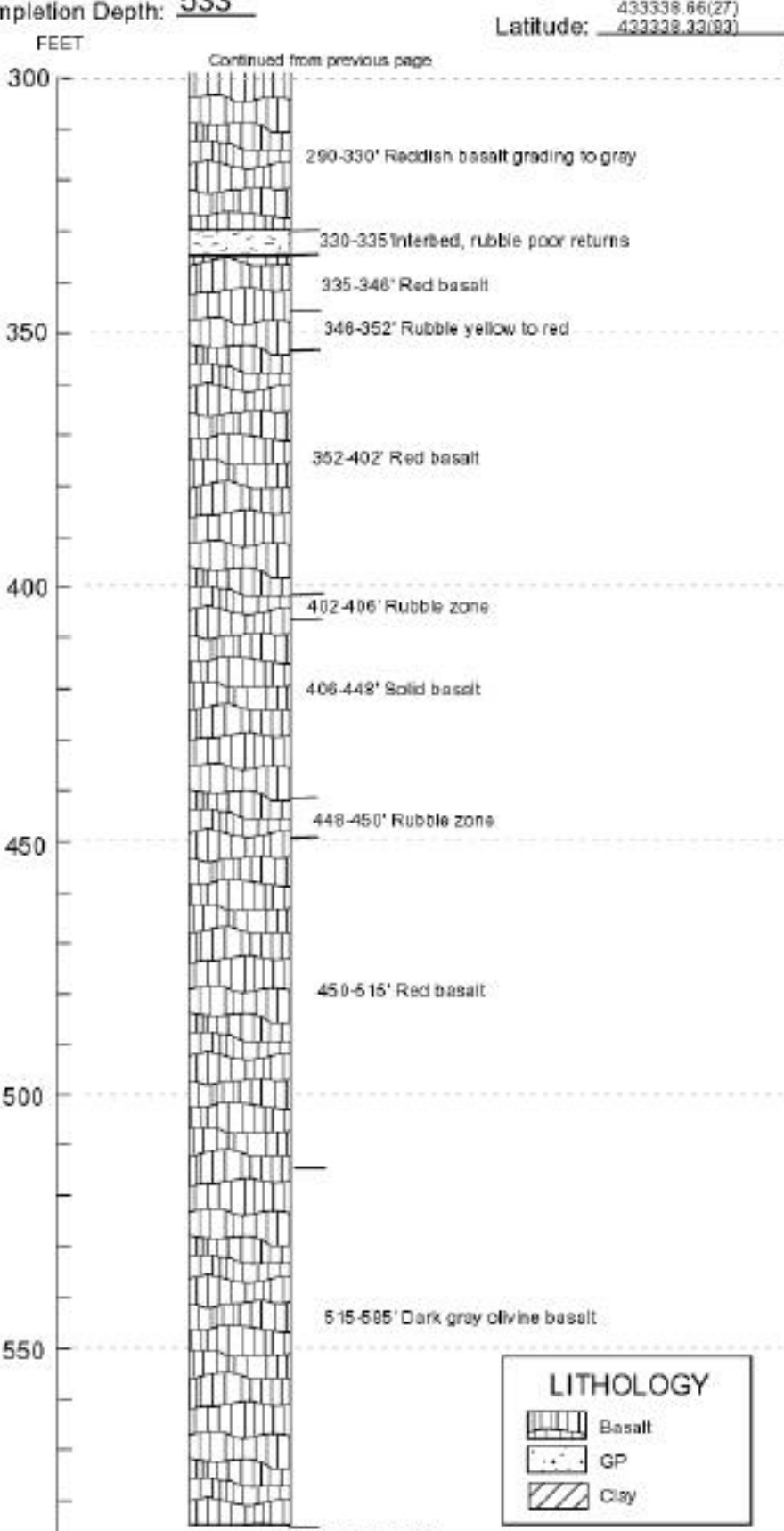

Tobal cepplit 505:

${ }^{G P}$

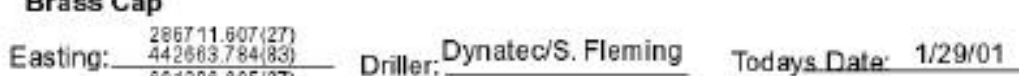

Water Level: $n$

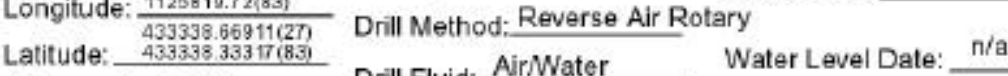

Measuring Point Drill Fluid: AirNater

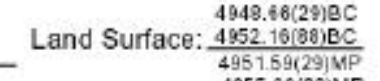

Water Level Access: n/a

$\underset{\text { APF-GR }}{\text { Natural Gamma }}$

$\underset{\text { API.N }}{\text { Neuton }}$

Gamma Gamma

Caliper
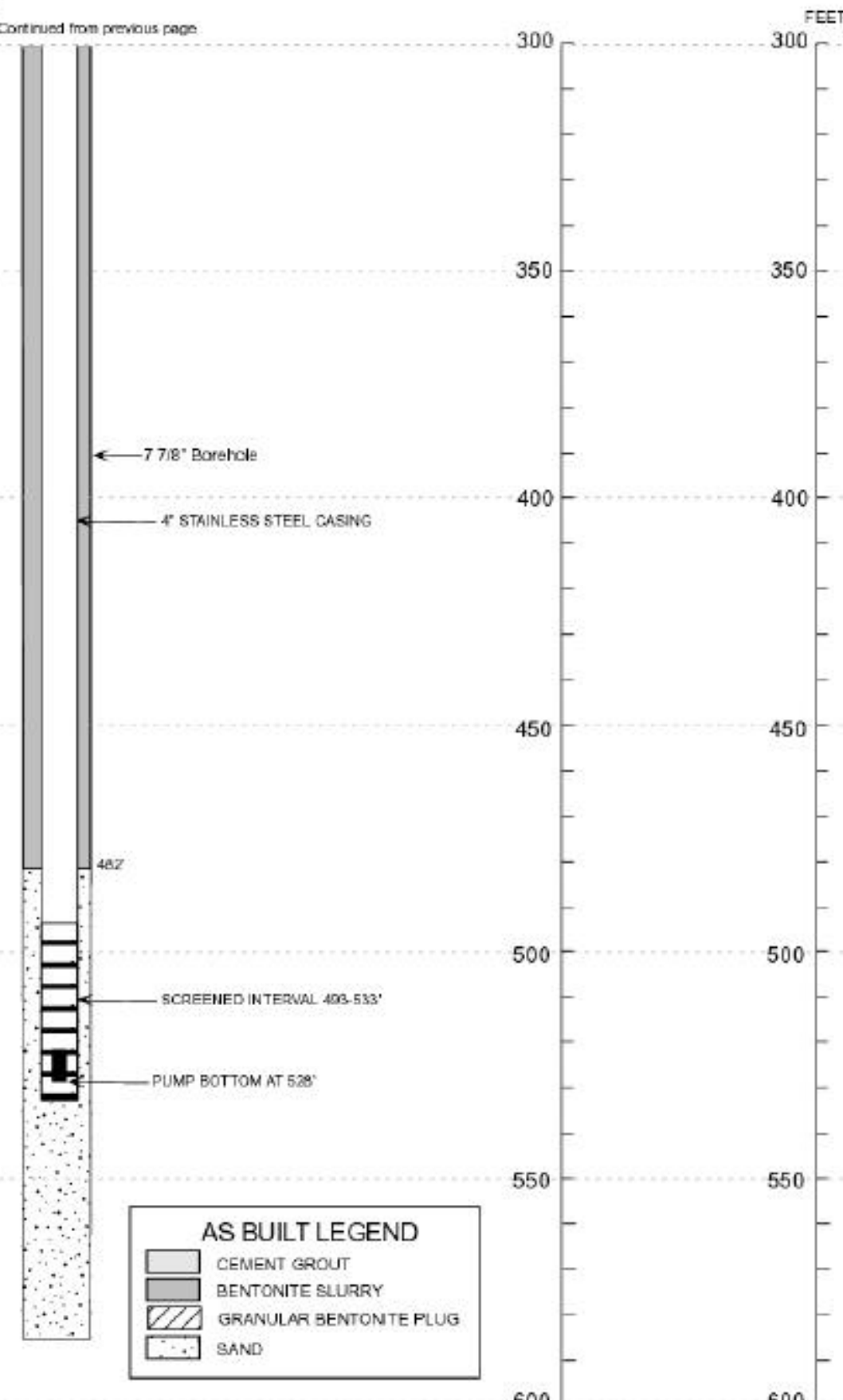


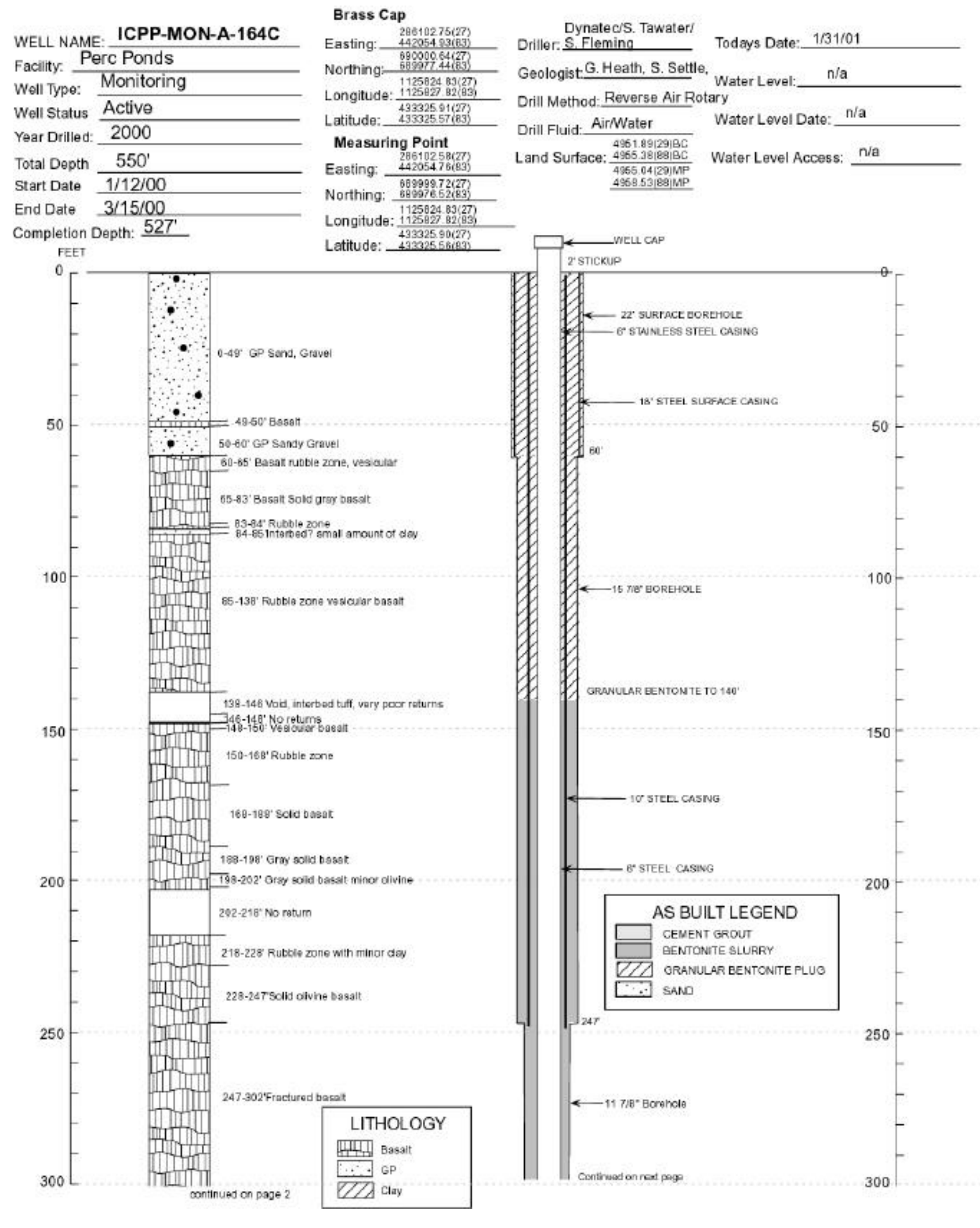

ICPP-MON-A-164C

$\underset{\substack{\text { Natural Gamma } \\ \text { API-GR }} \quad \text { Neutron }}{\text { GPINN }}$

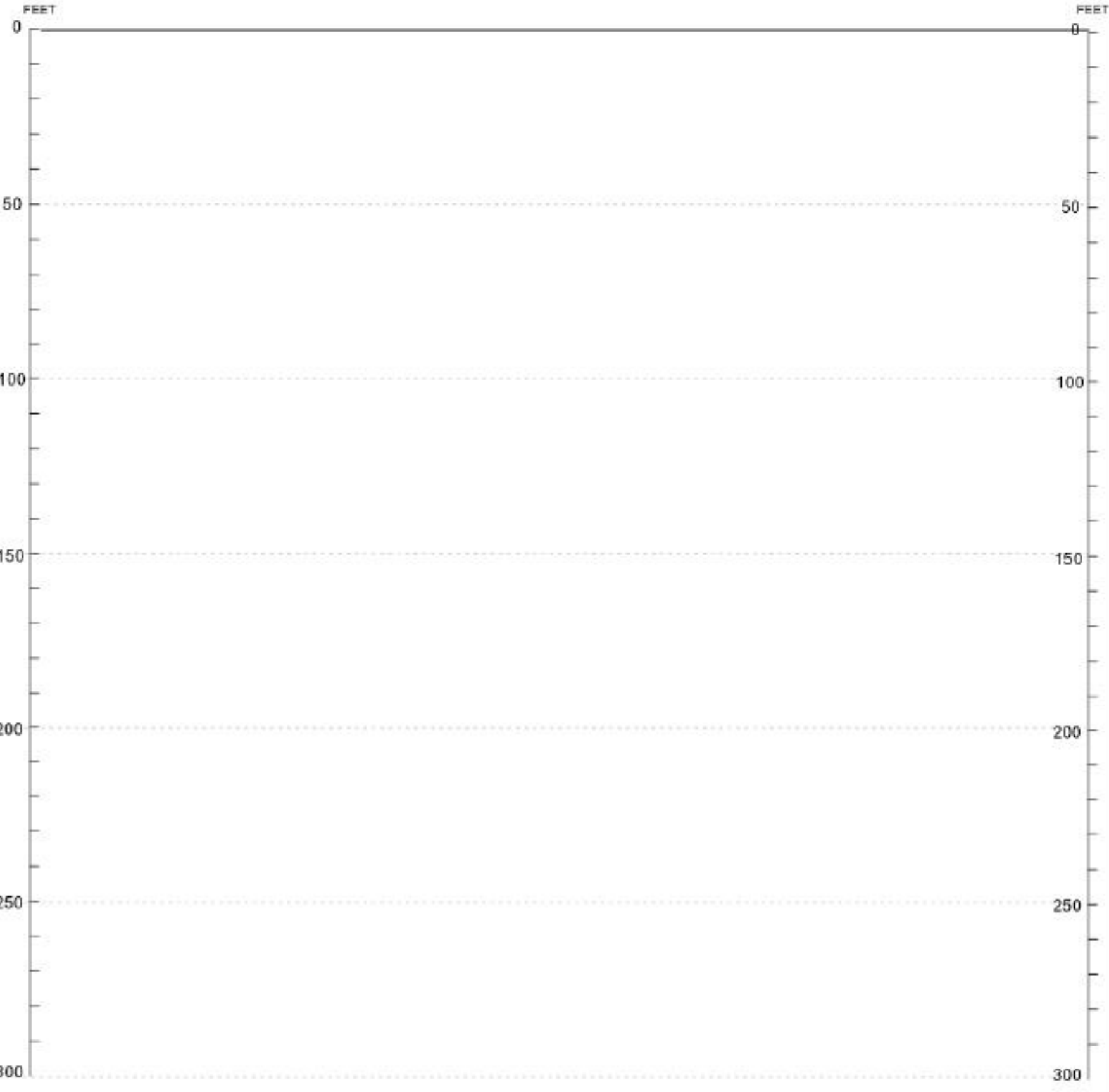


ICPP-MON-A-164C

WELL NAME: page 2

Well Type: Monitoring

Well Status Active

Year Drilled: 2000

Total Depth $550^{\circ}$

Start Date $1 / 12 / 00$

Completion Depth: $\underline{527^{\prime}}$
Brass Cap

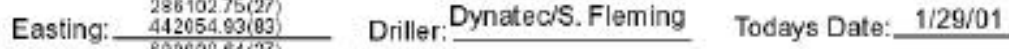

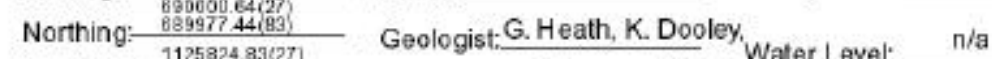

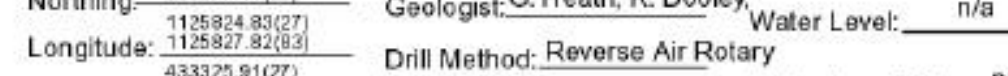

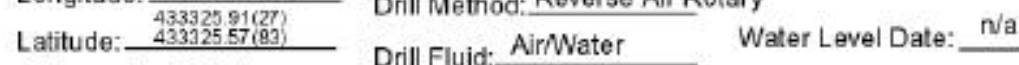

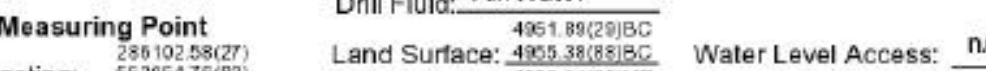

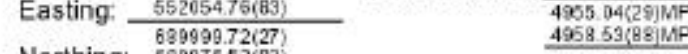

Northing: $\frac{089970.02(83)}{11253243927}$

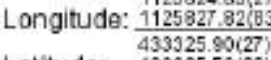

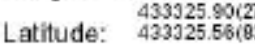

\section{ICPP-MON-A-164C}

$\underset{A P I-G R}{\text { Natural Gamma }}$ $\underset{\text { NFi-N }}{\text { Neutron }}$

$\underset{\text { Gamma Gamma }}{\text { cos }}$

Caliper
INCH

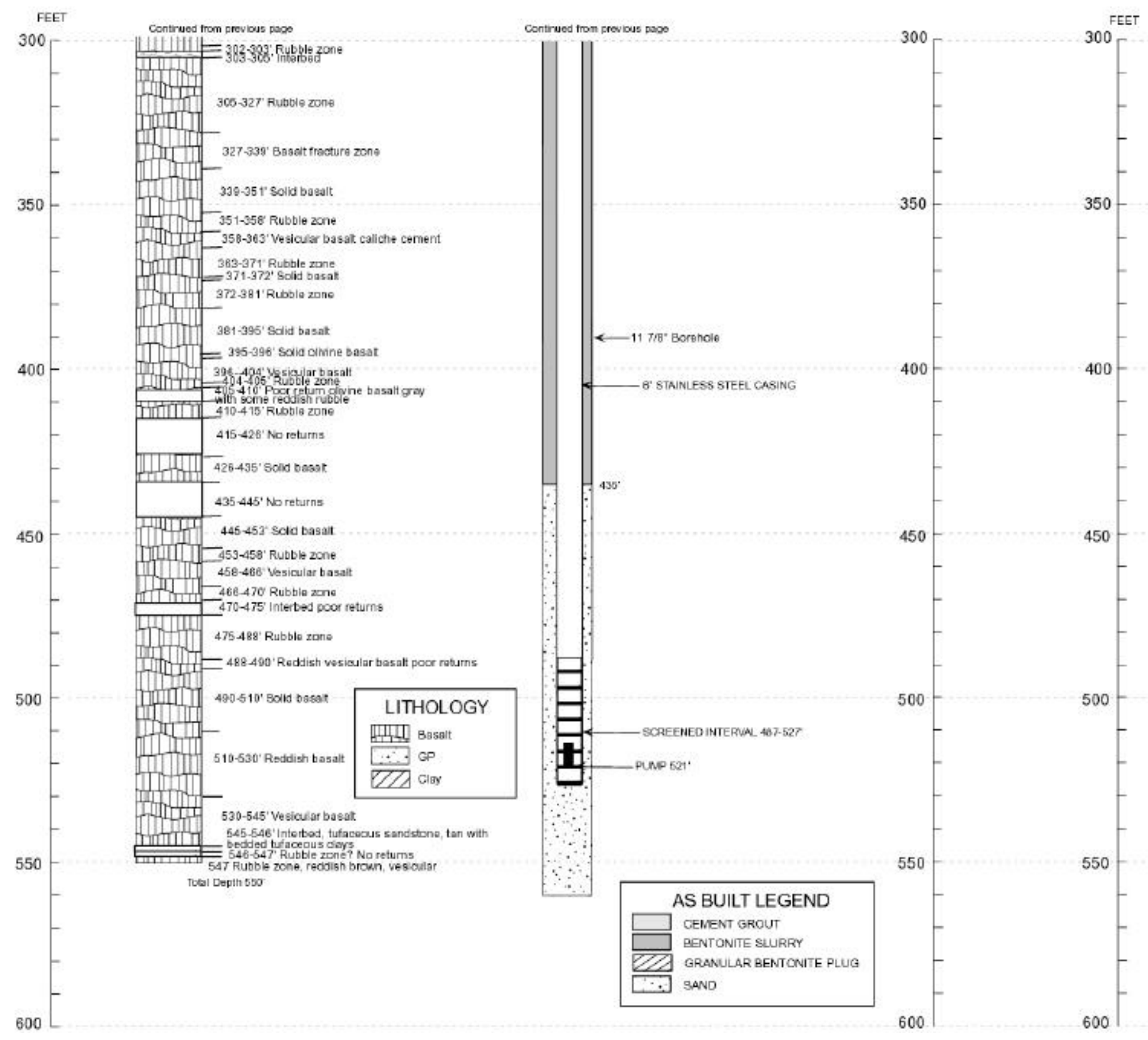


WELL NAME: ICPP-MON-A-165 Facility: $\frac{\text { Perc Ponds }}{\text { Monitoring }}$

Well Type: Monitoring

Well Status Active

Year Drilled: 2000

Total Depth $\frac{55}{4 / 1400}$

$5 / 1,00$

Completion Depth: $525^{\prime}$

Completion
FEET

Brass Cap

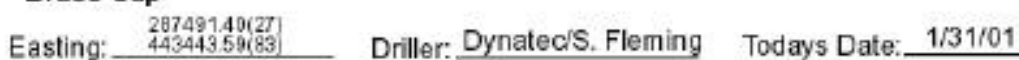

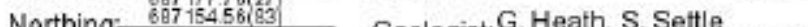

$489^{\prime}$

Drill Method: Reverse Air Rotary $5 / 3 / 00$

Drill Fluid: AirNater

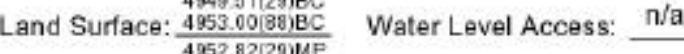

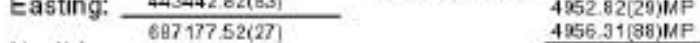

Northing: $\frac{667154326(63)}{1125855(51273)}$

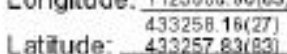
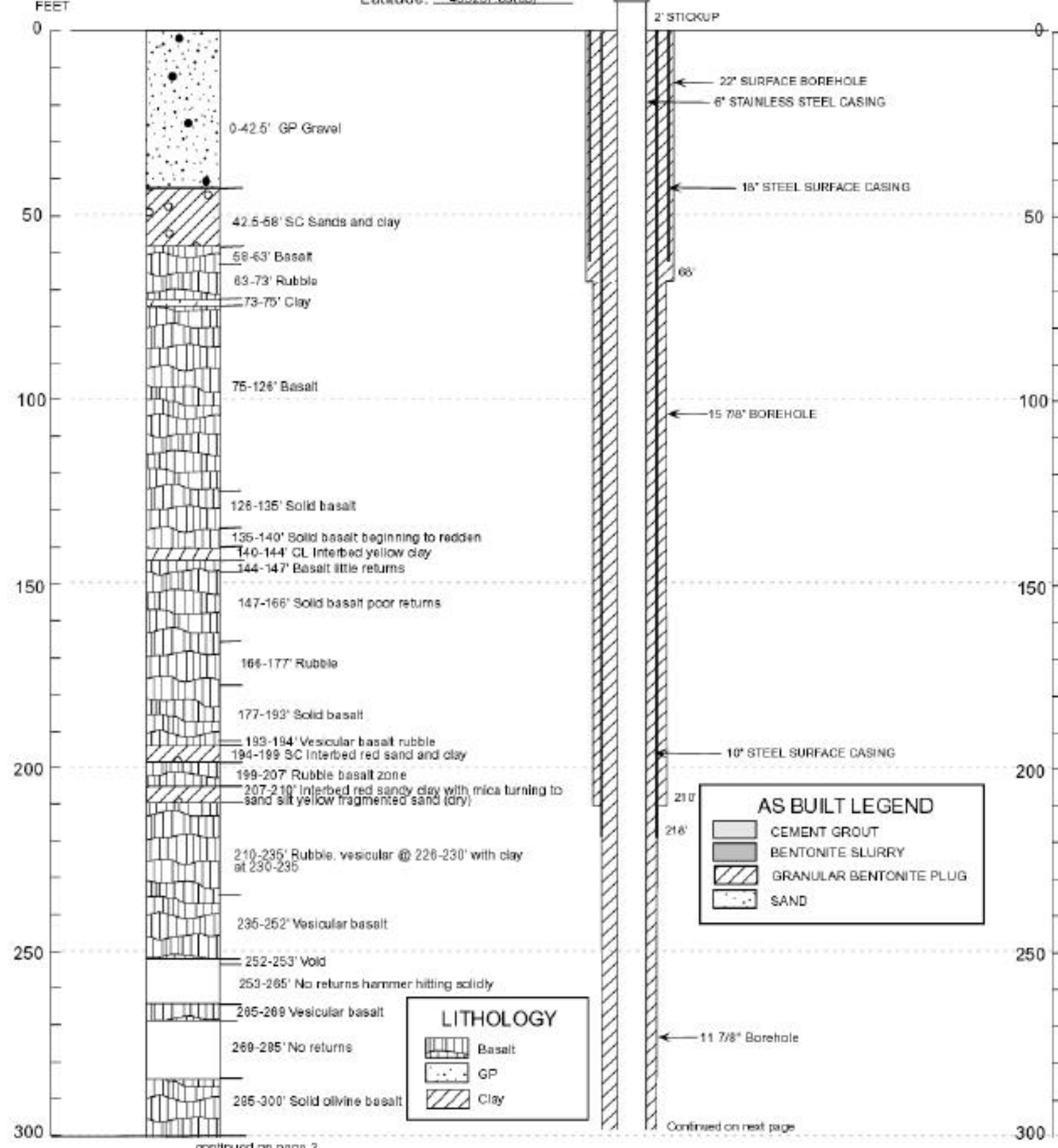

300

\section{ICPP-MON-A-165}

Natural Gamma

Neutron

$\underset{\text { CPS }}{\text { Gamma Gamma }}$

Caliper
INOS

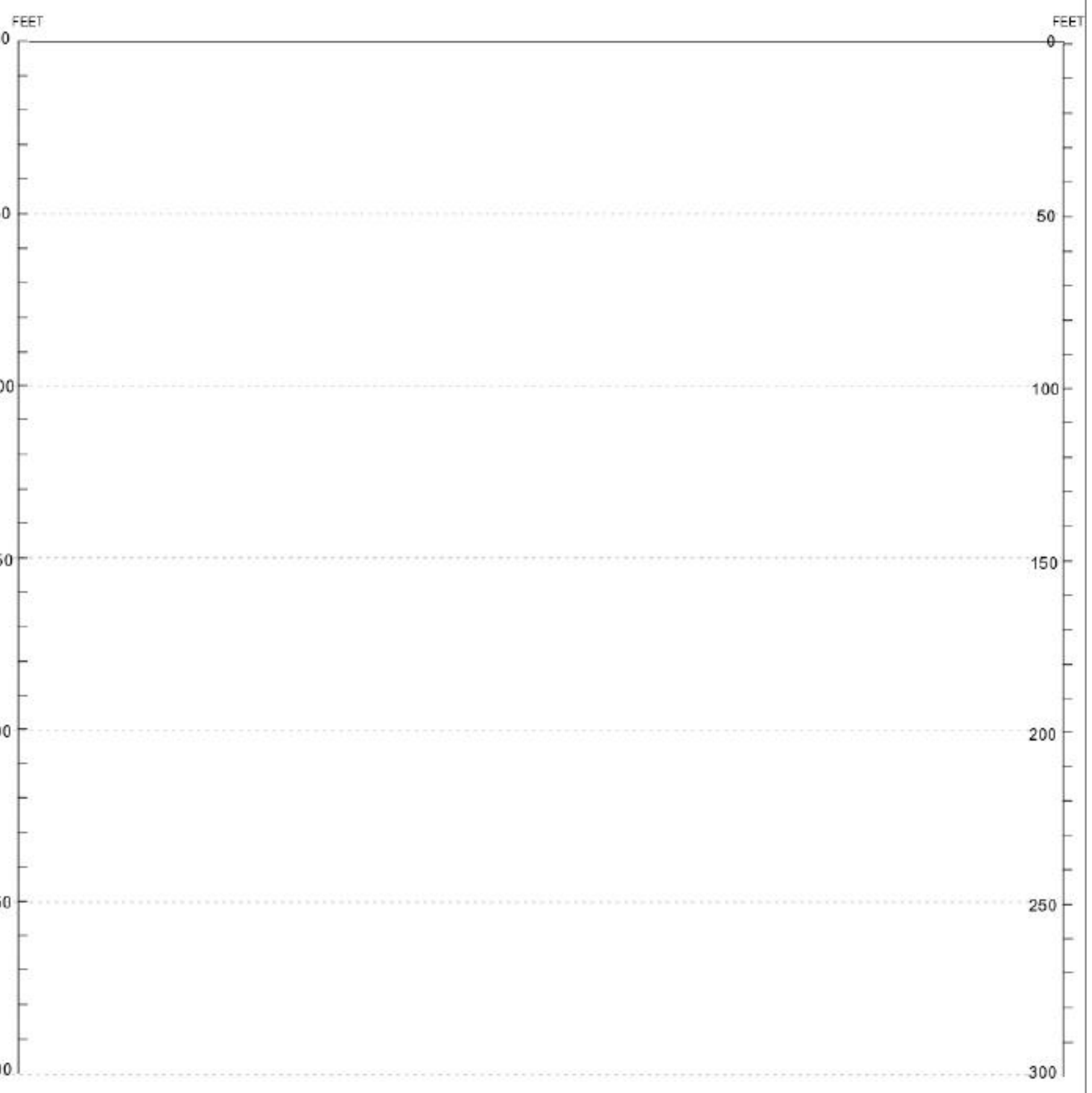


WELL NAME: Pacility: Perc Ponds

Well Type: Monitoring

Well Status Active

Year Drilled: 2000

Total Depth $555^{\circ}$

Start Date $4 / 14 / 00$

Completion Depth.

Brass Cap

Easting 1449443598(63) Driller: DynateolS. Flaming Todays Date: 1/31101

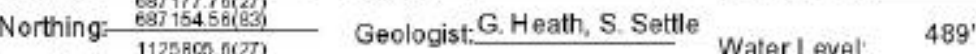

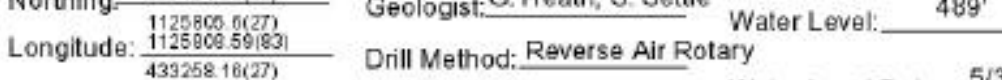

Longlude. 432925896253

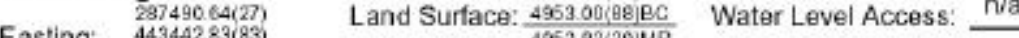

(697177522727)

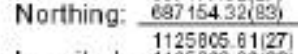

$\frac{1}{43225818(27)}$
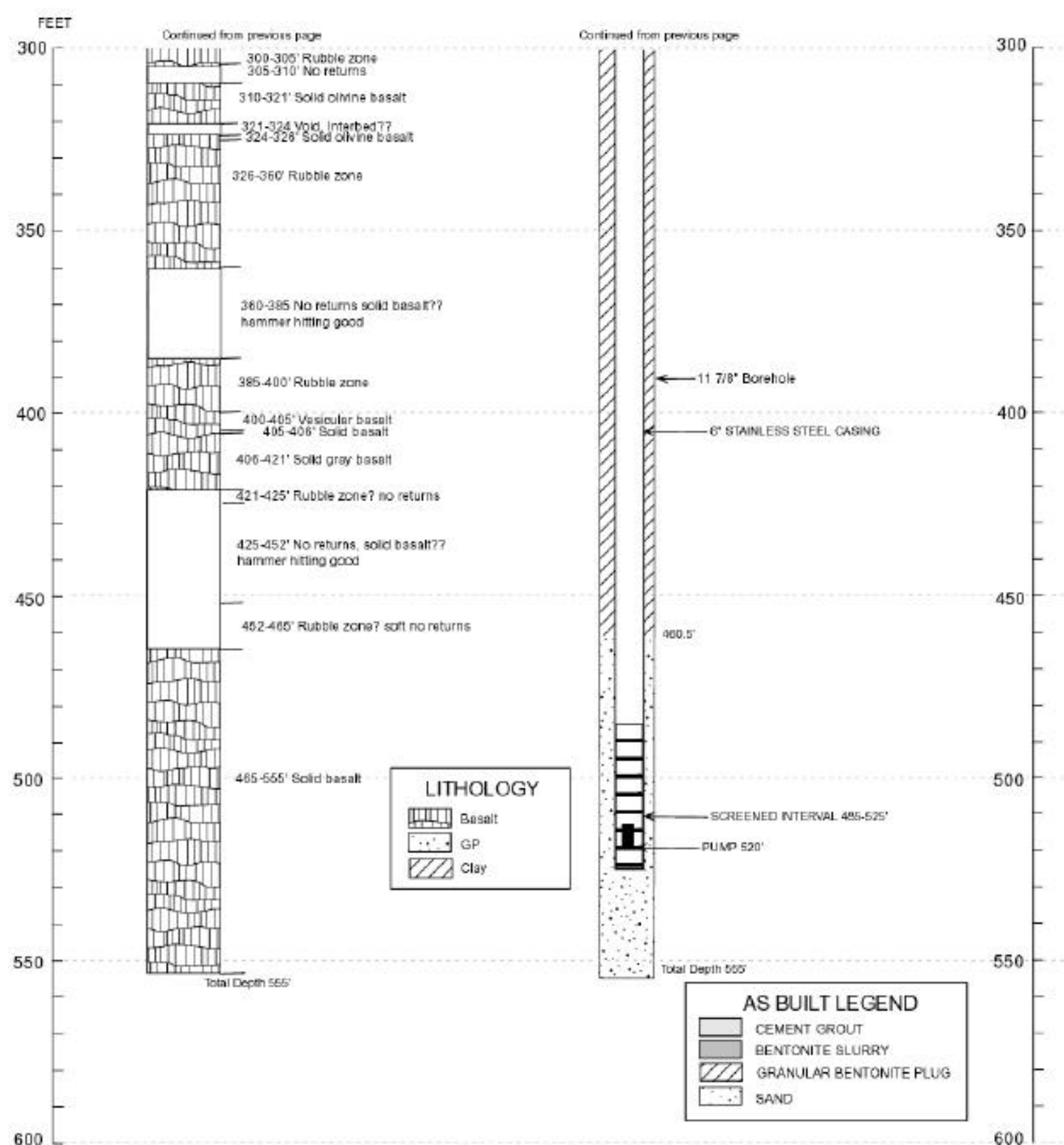

\section{ICPP-MON-A-165}

Natural Gamma 
WELL NAME: ICPP-MON-A-166 Facility: Perc Ponds Well Type: Monitoring Well Status Active Year Drilled: 2000

Total Depth $547^{\prime}$

Start Date $3 / 23 / 00$

End Date 4/7/00

Completion Depth:

Brass Cap

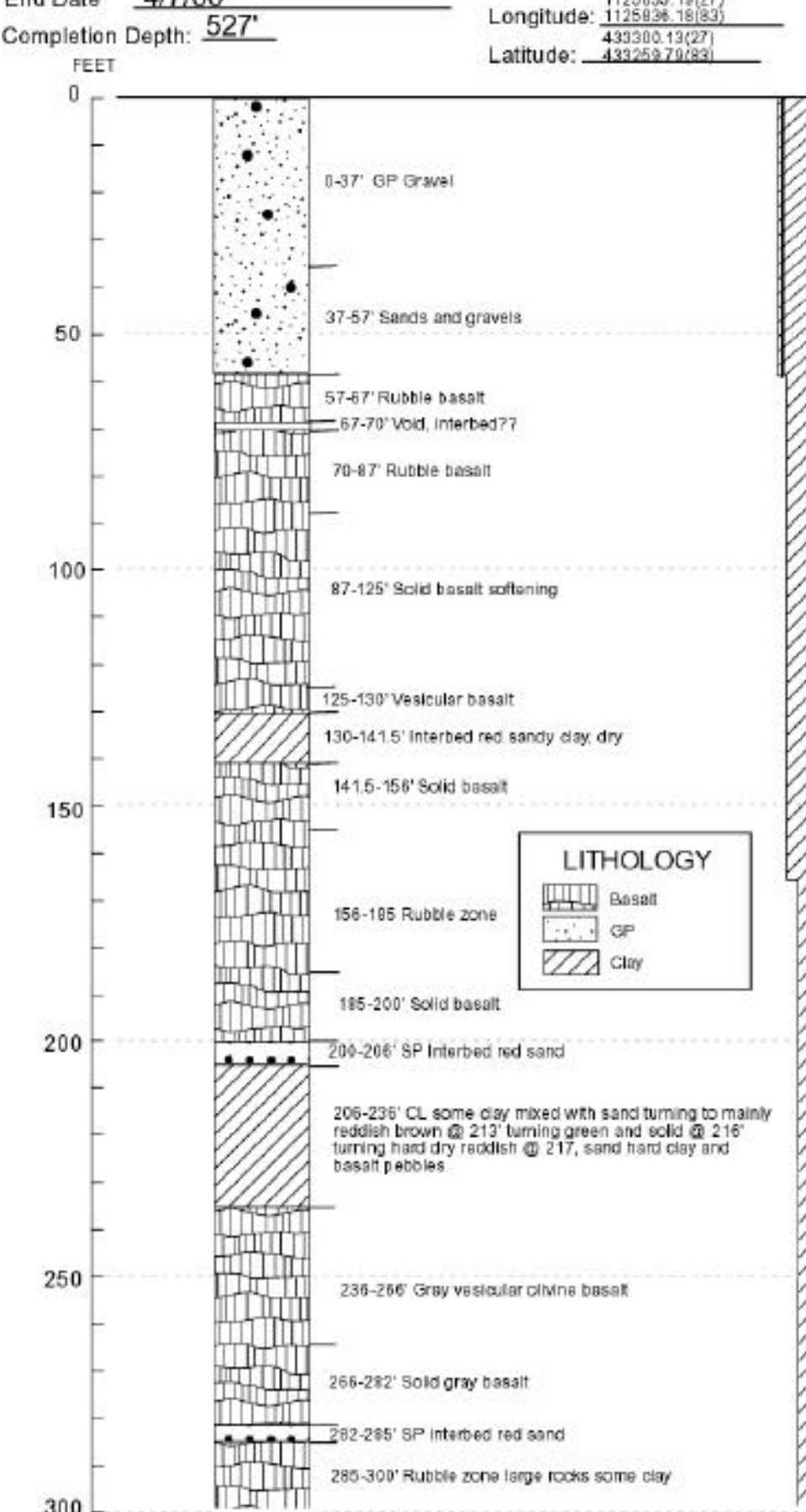

continued on Doge 2 687398.00(27)

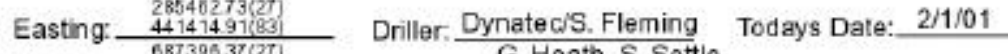

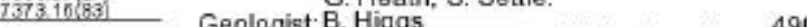
Water Level: $490^{\circ}$

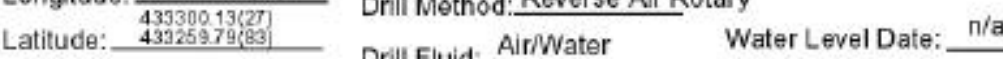

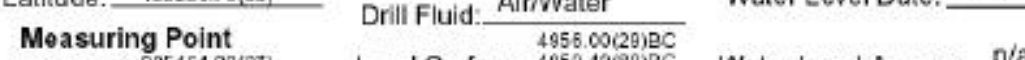

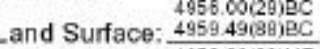
Water Level Access:

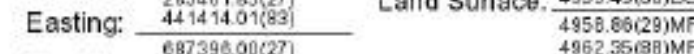
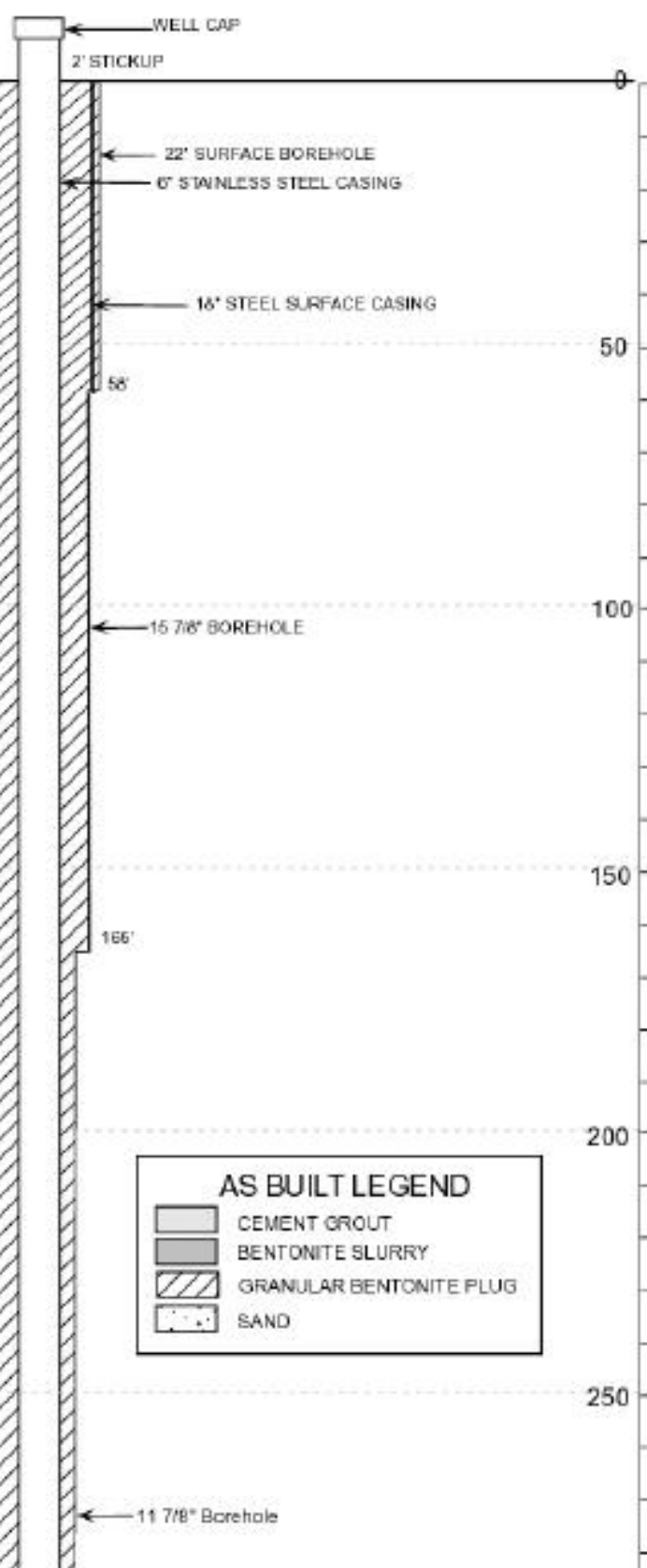

ICPP-MON-A-166

Natural Gamma

$\underset{\text { API.N }}{\text { Neutron }}$

Gamma Gamma

Caliper

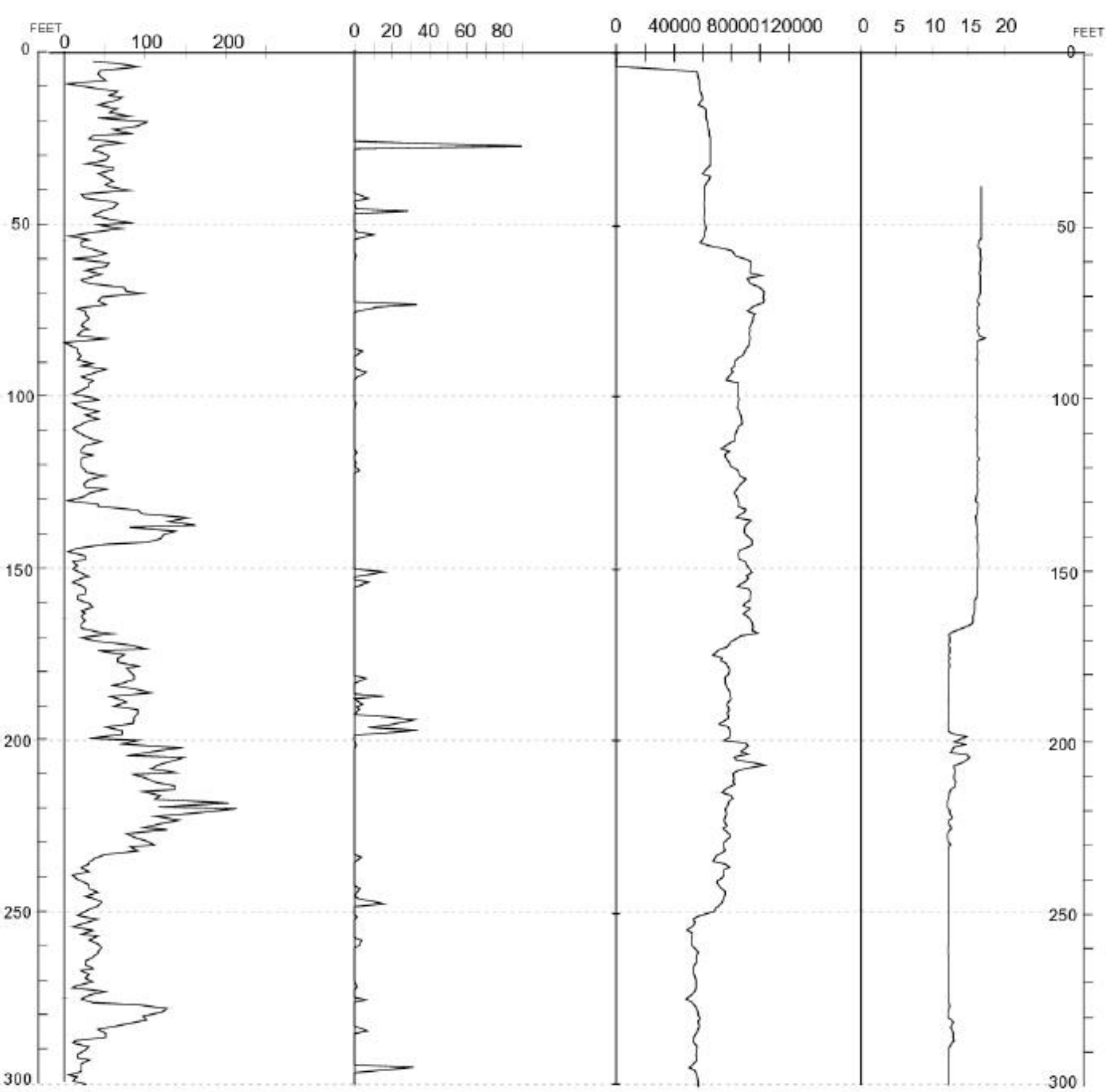


ICPP-MON-A-166
page 2

WELL NAME: Page 2

Facilly: Perc Ponds

Brass Cap

Watar Level 400

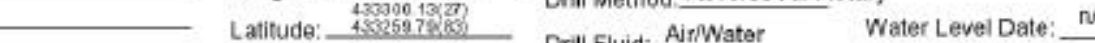

total Depth 547

Start Date $3 / 23 / 00$

End Date $4 / 7 / 100$

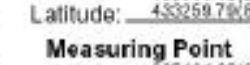

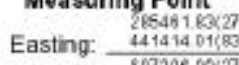

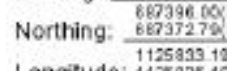

1560.001298BC

Water Level Access: n/a

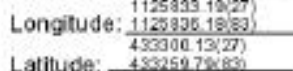

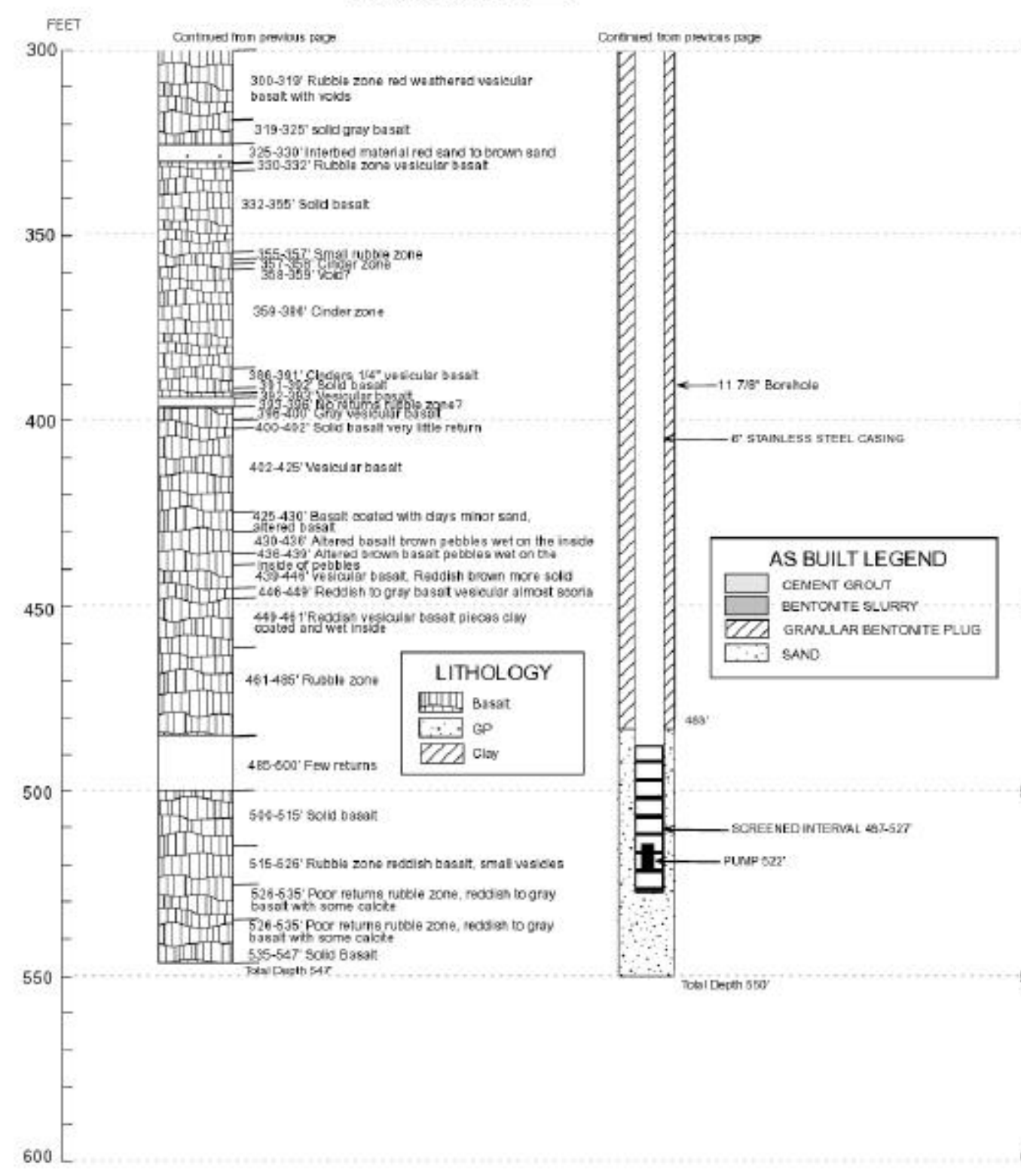

\section{$\underset{\text { Page } 2}{\text { ICPP-AN-A-166 }}$}

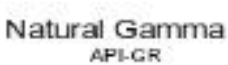

$\underset{\substack{\text { Neutron } \\ \text { AP-N }}}{ }$

$\underset{\mathrm{CPB}}{\operatorname{Gamma}}$

Caliper

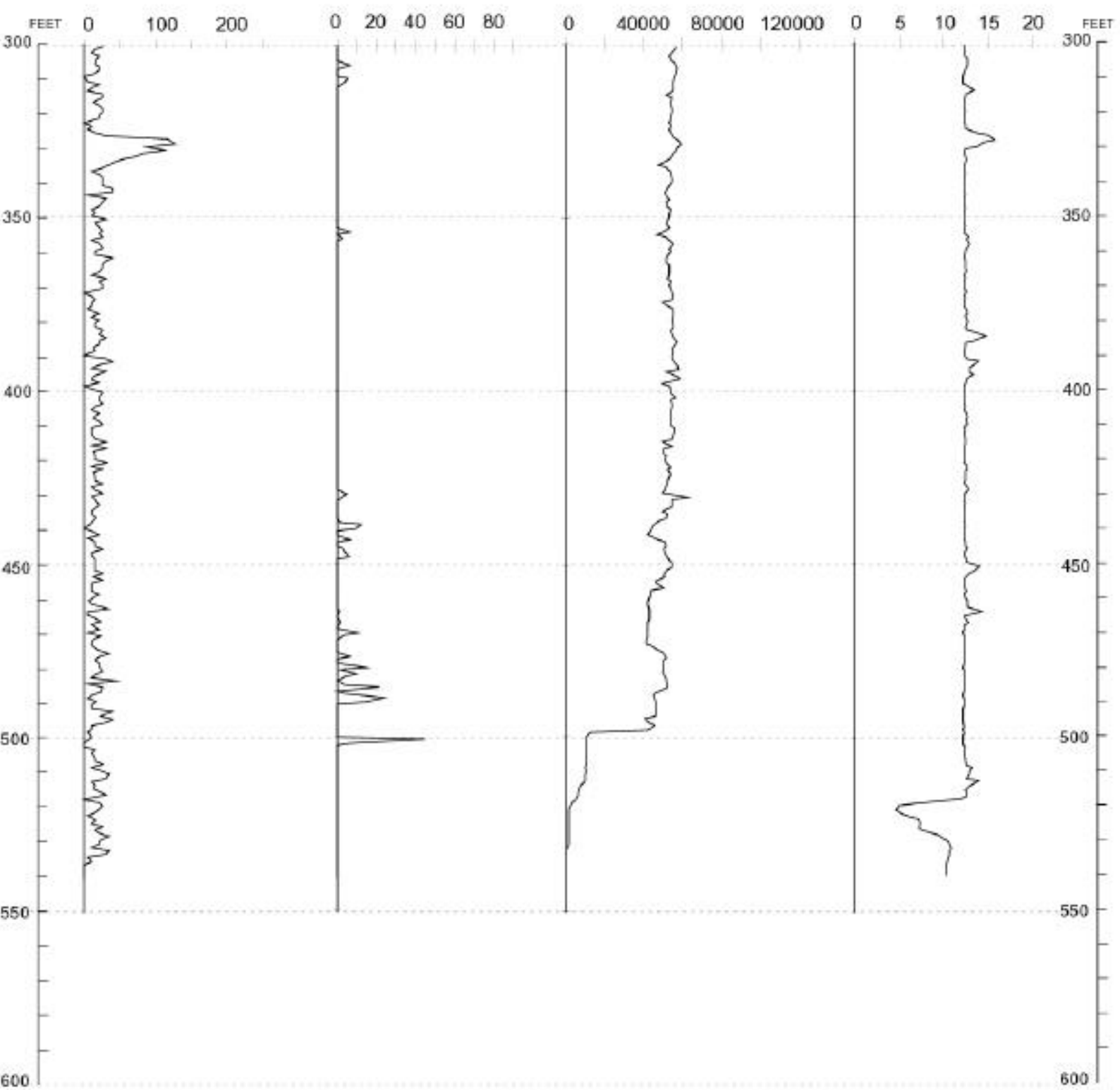


WELL NAME: ICPP-MON-A-167

Facillty: Perc Ponds

Well Status Active

Year Drilled: 2000

Total Depth $525^{\circ}$

Start Date $5 / 16 / 00$

End Date $6 / 8 / 00$

Completion Depth: $502^{\prime}$

Brass Cap

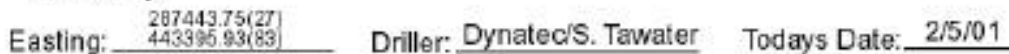

Northing: 69c4a435t(3) Geologist.G. Heath. S. Settle

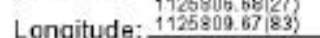

Drill Method: Reverse Air Rotary

Drill Fluid: Water

6her $6 / 8 / 0$

Measuring Point

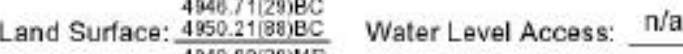

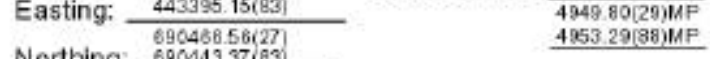

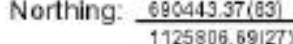

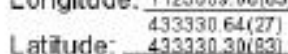
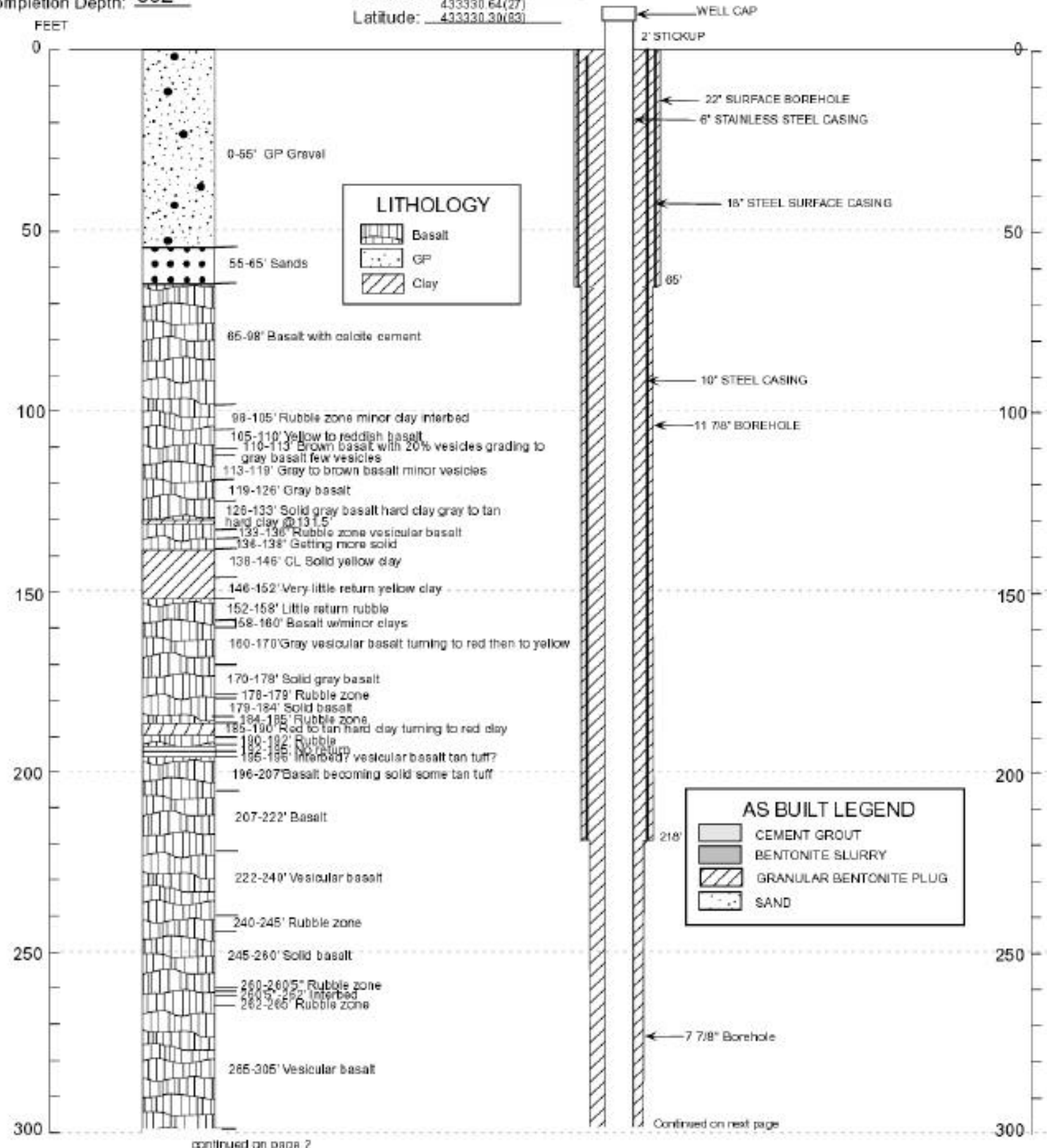

\section{ICPP-MON-A-167}

Natural Gamma

Neutron

$\underset{\mathrm{CPS}}{\operatorname{Gamma} \text { Gamma }}$

Caliper
INOS

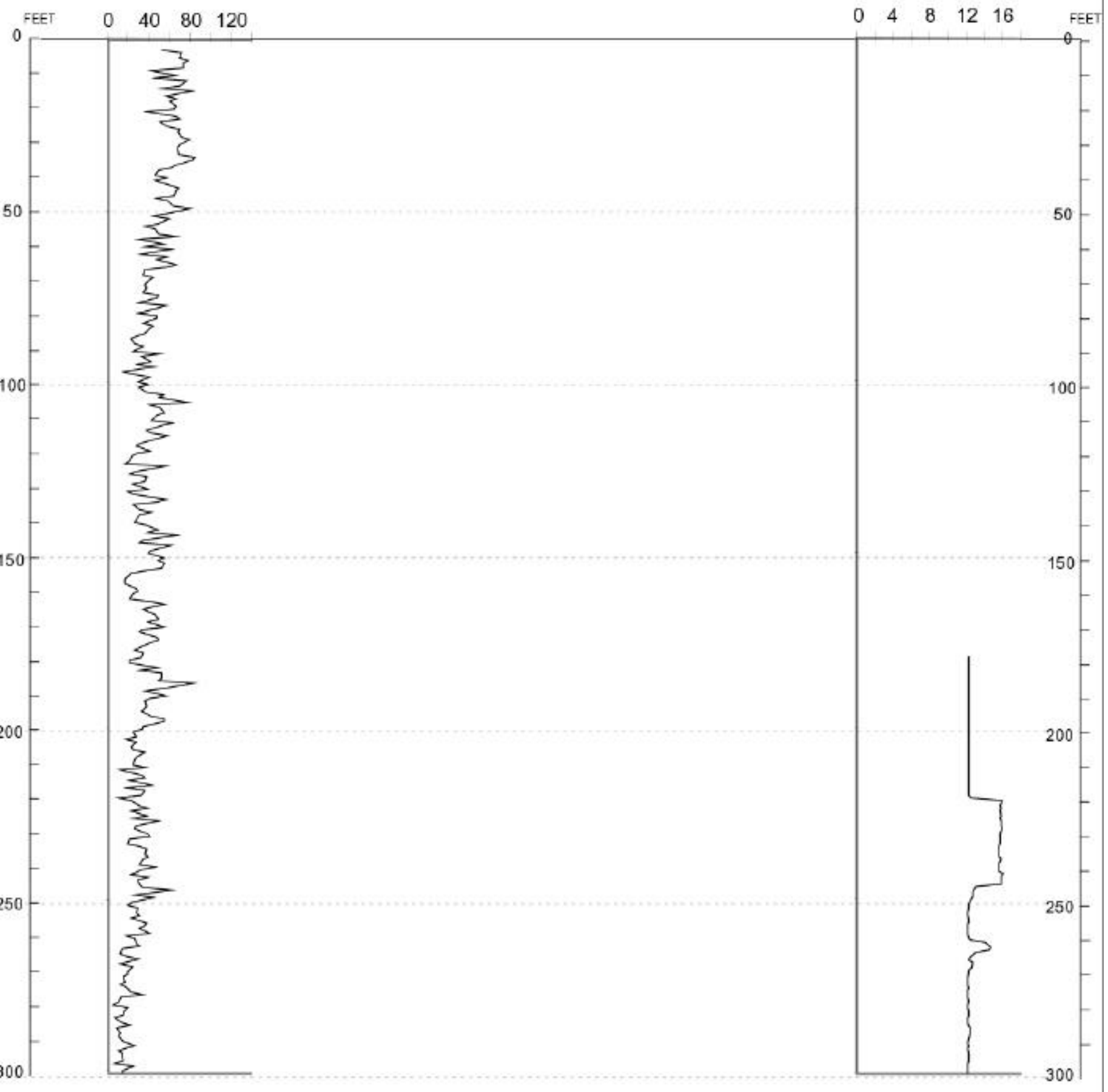




\begin{tabular}{|c|c|}
\hline \\
\hline \\
\hline \multirow{2}{*}{\multicolumn{2}{|c|}{$\begin{array}{l}\text { Well Status } \\
\text { Year Drilled: }\end{array}$}} \\
\hline & \\
\hline \multicolumn{2}{|c|}{$\begin{array}{l}\text { Year Drilled: } \\
\text { Total Depth }\end{array}$} \\
\hline Start Date & $5 / 16 / 00$ \\
\hline \multicolumn{2}{|c|}{ End Date $\quad \frac{6 / 8 / 00}{50}$} \\
\hline
\end{tabular}

Brass Cap

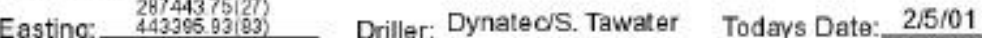

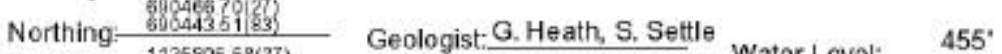

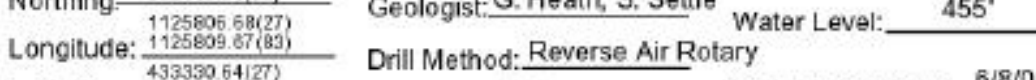

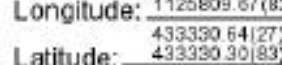
Latitude: 43335030 Drill Fluid: Water Water Level Date: 6/8180 Measuring Point

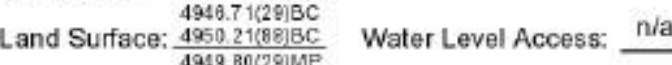

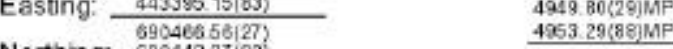
Northing: $\frac{690443.37837}{11258925693(27)}$

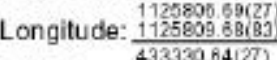

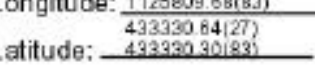

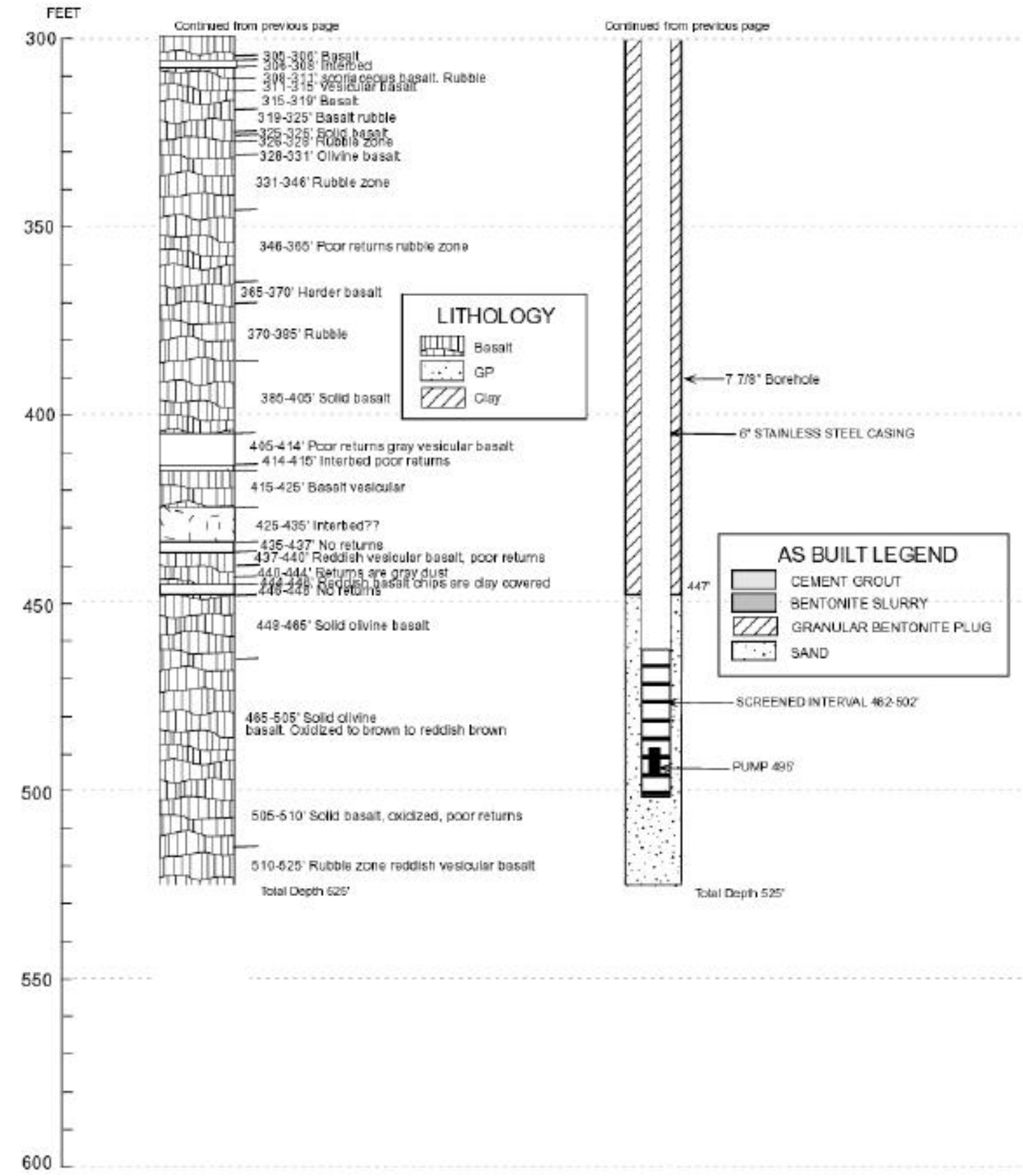

\section{$\underset{\text { ICPP-MON-A-167 }}{\text { page } 2}$}

Natural Gamma

$\underset{\substack{\text { Neutron } \\ \text { AF-N }}}{\cos }$

$\underset{C \text { Gamma Gamma }}{\text { Gam }}$

Caliper

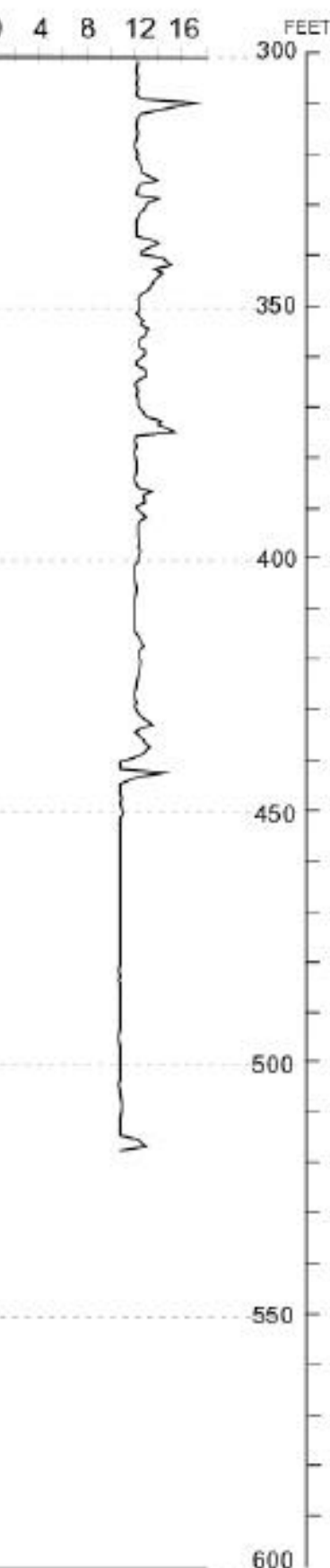


Brass Cap

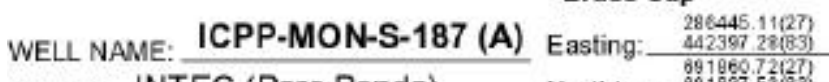

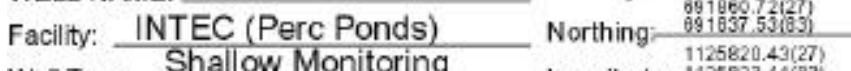

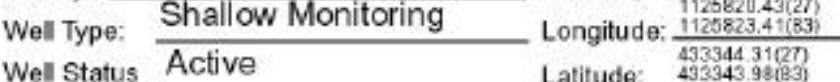

Wel Status $\frac{\text { Active }}{2000}$

Total Depth $\frac{49.4^{\prime}}{9 / 21 / 00}$

Driller. Dynateo'Roy Brown Todays Date: 1/8/01 Geologist: G. Oberhansley Water Level: na Me asuring Point

Drill Method: Reverse Air Rotary $\quad$ Water Level Date: na

Dill Fluid: Air Easting: $442396984(3)$

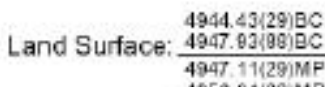

Water Level Access: na

End Date 9/22/00

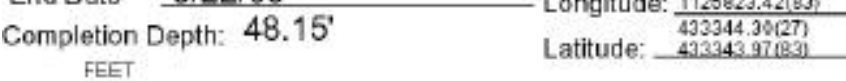

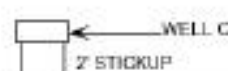
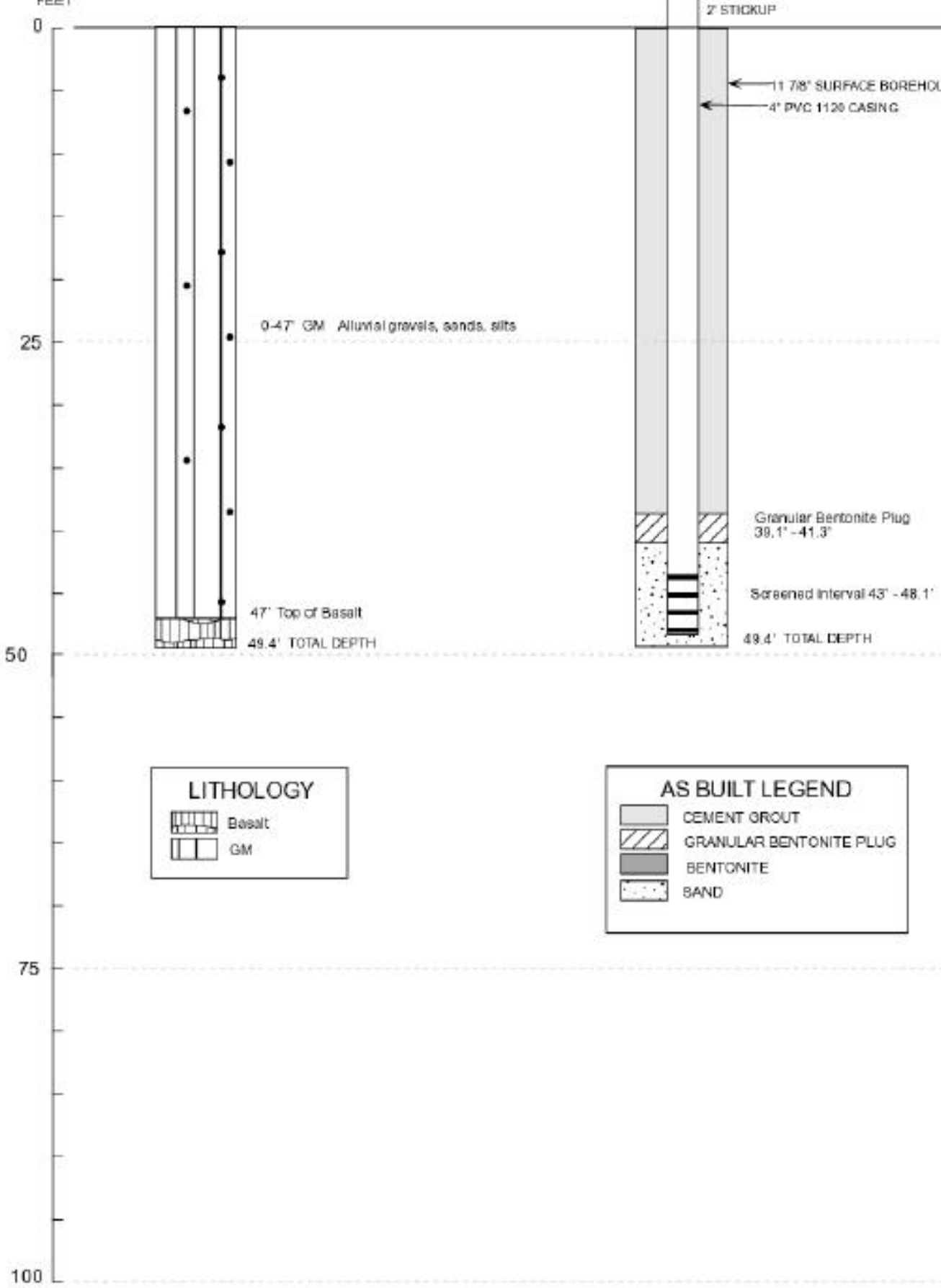

LITHOLOG

피밈
Natural Gamma

$\underset{\substack{\text { Neutron } \\ \text { APIN }}}{\cos }$

$\underset{c \operatorname{cPS}}{\text { Gamma Gamma }}$

$\underset{\substack{\text { Caliper } \\ \text { INCH }}}{\text { and }}$

$0^{\text {FEET }}$

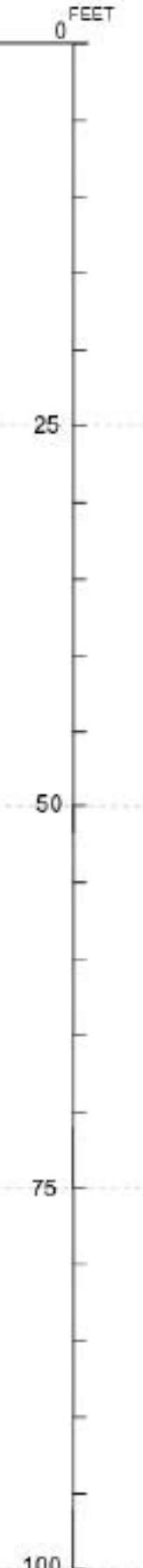

$0^{\text {FEET }}$

50

$50-$

75

$100 \mathrm{~L}$ 
WELL NAME: ICPP-MON-V-188 (C) Facility: INTEC (Perc Ponds) Well Type: Monitoring

Well Status Active

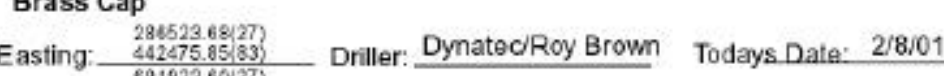

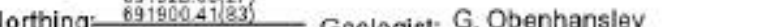

ing

Year Drilled: 2000

Total Depth $121^{\prime}$

Start Date 9/12/00

End Date $\frac{9 / 14 / 00}{119}$

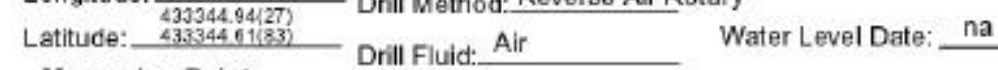

Measuring Point 265235 (27) Dril

na

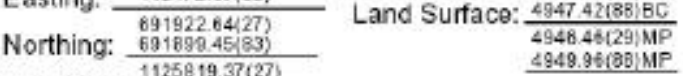

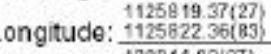

Completion Depth: 119.8'

100

200

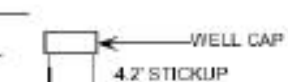

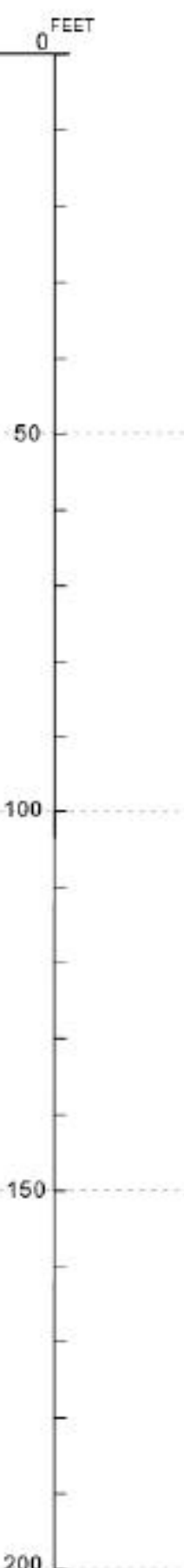

\section{ICPP-MON-V-188 (C)}

Natural Gamma

$\underset{\substack{\text { Neutron } \\ \text { API.N }}}{\cos (2)}$

Gamma Gamma

Caliper

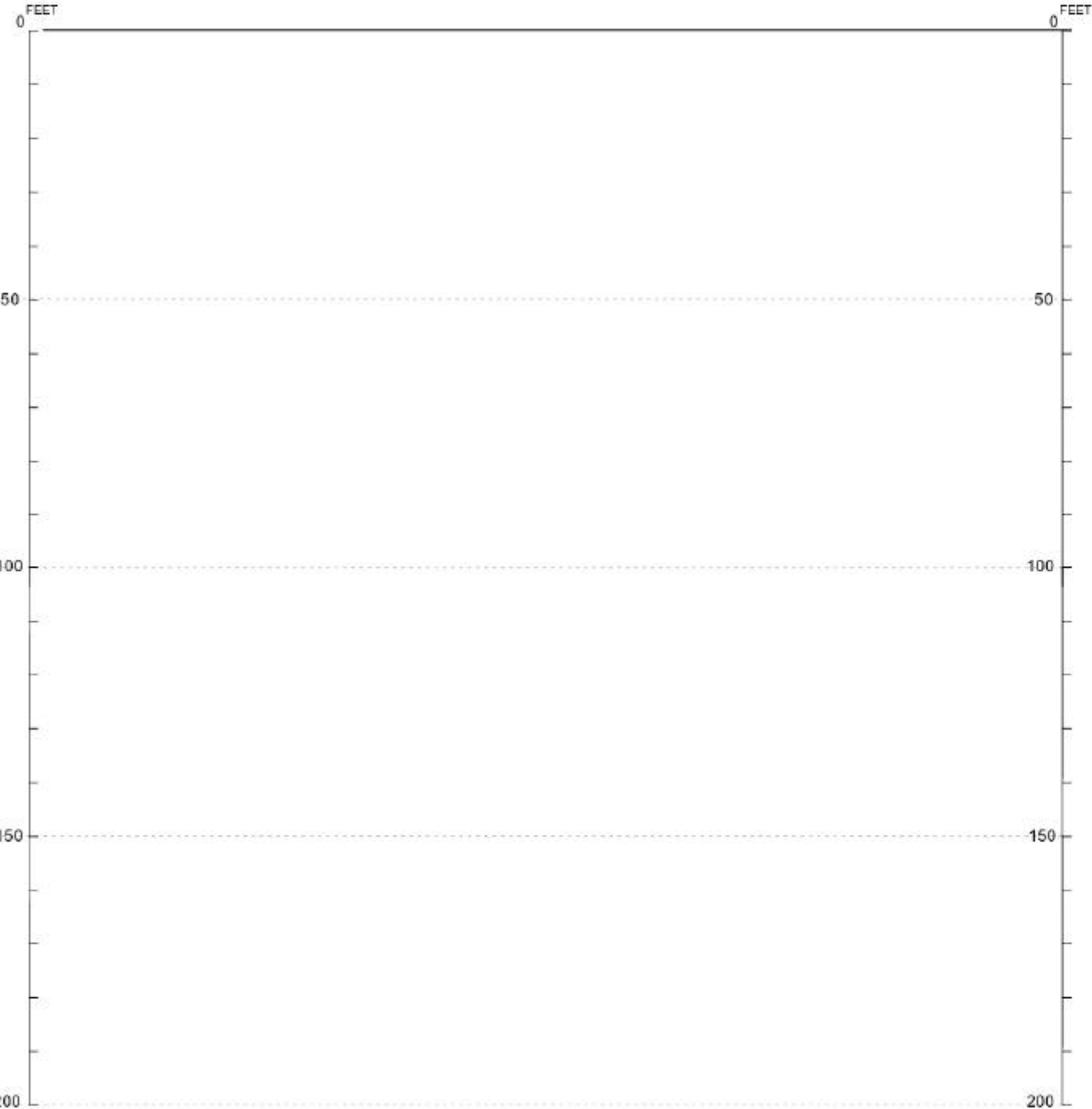




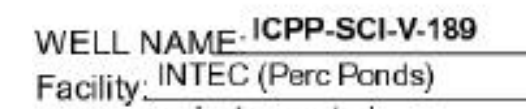

Brass Cap

Easting: 205480.8

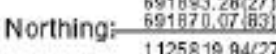

Driller: Dynatec/S. Tawater

Geologist: G. Oberhansley

Water Level: na

Well Statusctive

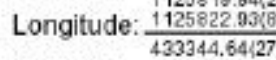

Drill Method Reverse Air Rotas

Year Drilled2001

Measuring Point

4944.372029:8C

Water Level Date: na

Total Depth $153^{\prime 2}$

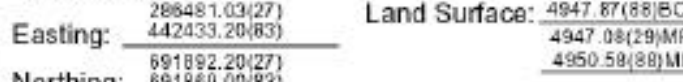

Water Level Access: na

End Date $12 / 22 \% 00$

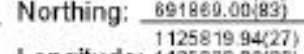

Completion Depth: 153'2"

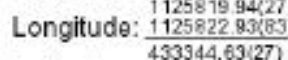

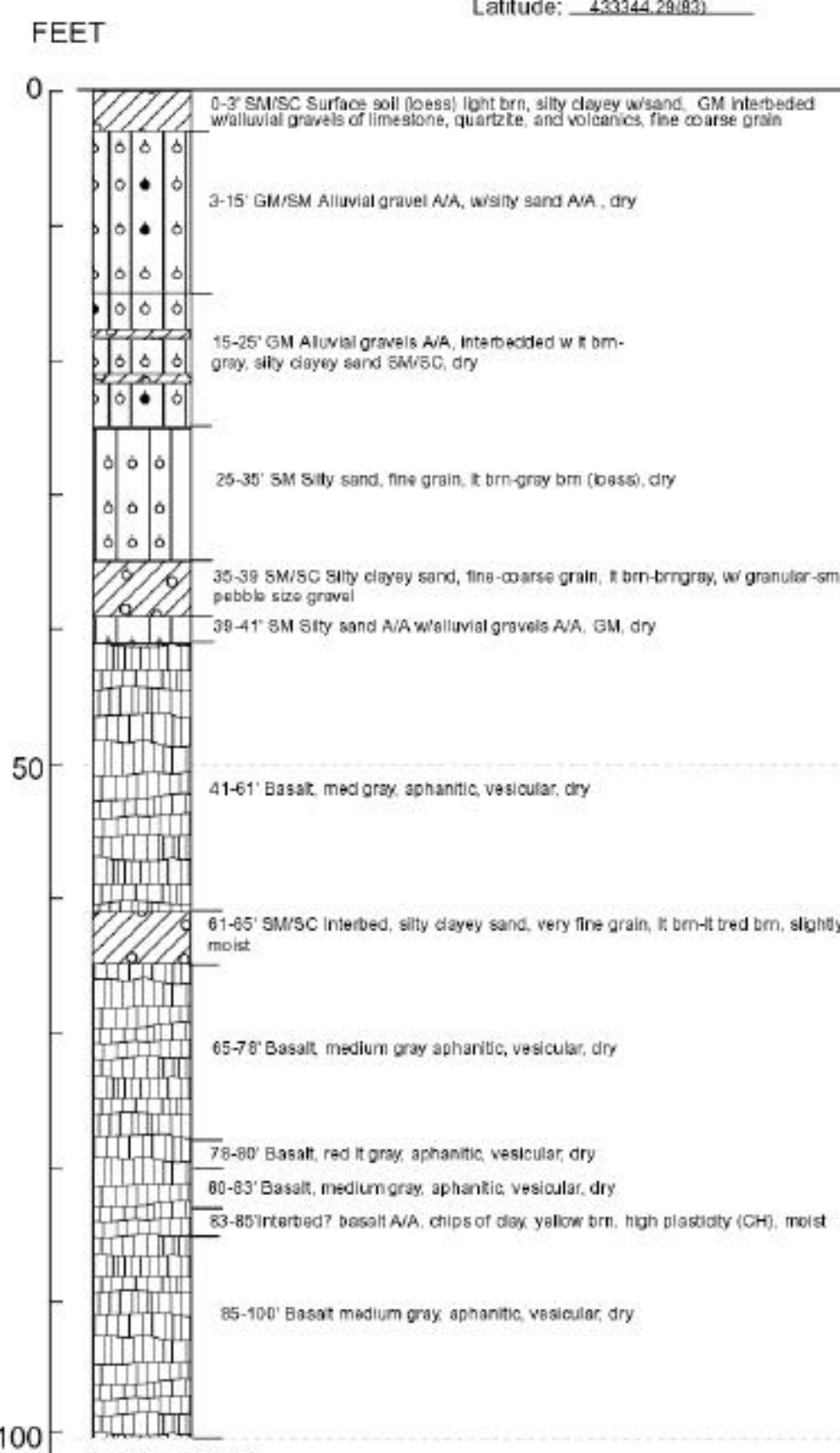

$100-$

Cominues on poso 2

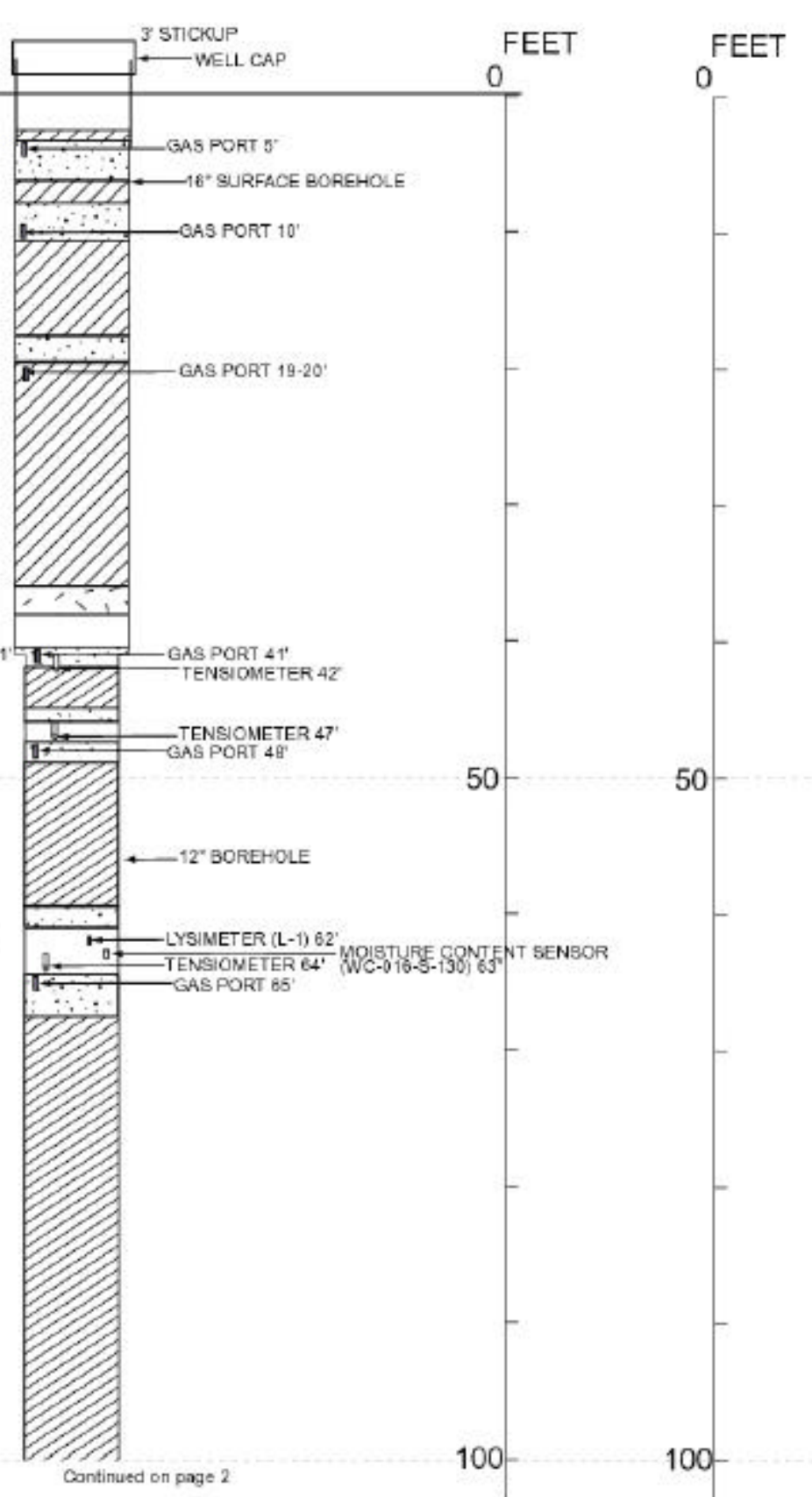

Natural Gamma

$\underset{\text { APPN }}{\text { Neutron }}$

$\underset{\mathrm{CPS}}{\operatorname{Gamma}}$

Caliper
INGH 
WELL NAME. ICPP-SCI-V-189 pg 2 Brass Cap

Facility: INTEC (Perc Ponds)

Well Type:Instrumented

Well Statustctive

Year Drilled2001

Total Depth 153'2"

Start Date $12 / 13 / 00$

End Date 12/22/00

Completion Depth: $153^{2} 2^{\circ}$

Easting: $\frac{264680.05}{44243128}$

Northing: 691989.280 .283$\}$

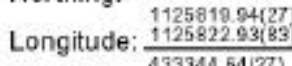

Driller: Dynatec/S. Tawater Todays Date: 6/19/01

Geologist: G. Oberhansley Water Level: na

Drill Method Reverse Air Rotary

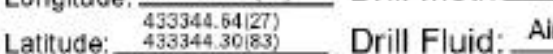

War Levi Date:

Measuring Point Easting: $\frac{442439.201233}{699189220277}$

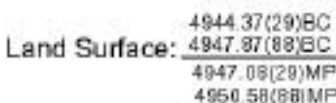

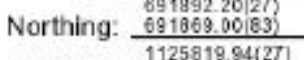

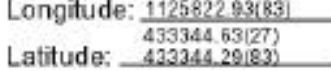

FEET

$$
\text { Continued trom prevevius page }
$$

FEET

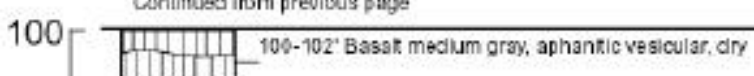

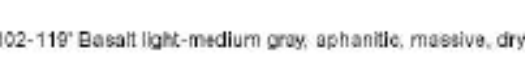

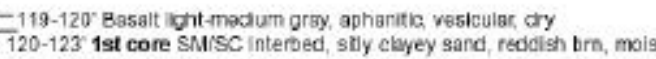

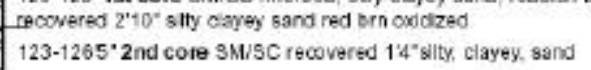

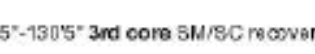

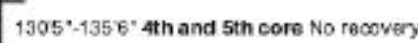

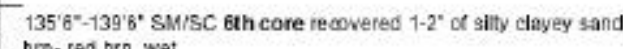

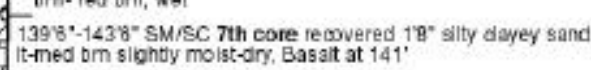

1.

$150-$

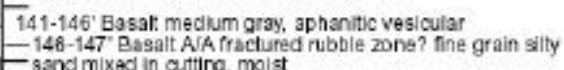

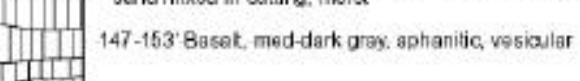

$$
\begin{aligned}
& \text { LITHOLOGY } \\
& \text { IIIII) Basat }
\end{aligned}
$$

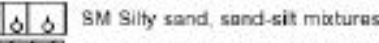

$$
\begin{aligned}
& 192 \text { swisc silty sands sand sit mixitur. }
\end{aligned}
$$

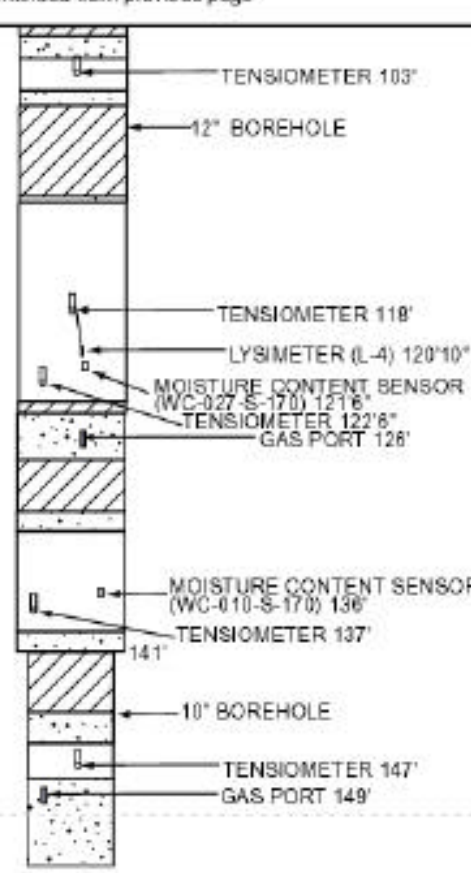$$
\text { AS BUILT LEGEND }
$$$$
\text { CDh GRAENT GROUT }
$$

BENTONITE SLURRY

$\because$ SAND

On. AlL (CANE)

DILICA FLOUR

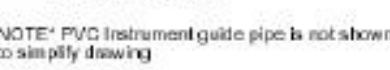


Brass Cap

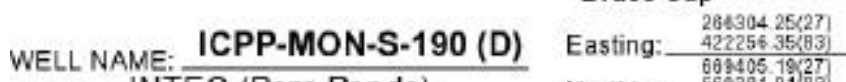

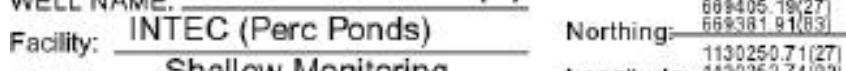
Shallow Monitoring Wellsp: Driller: DynatedRoy Brown Todays Date: 1/9/01 Year Drilled: 2000 Geologist Gater Level: na

To

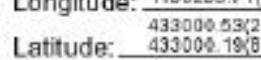
Drill Method:Reverse Air Rotary

Total Depth $\frac{40^{\prime}}{9^{\prime} 15{ }^{\prime}}$

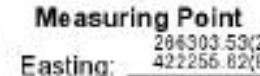
$\underset{\text { NPPLCR }}{\text { Natura }}$ 
WELL NAME: ICPP-MON-S-190A

Facility:

Well Type: Shallow Monitoring

Brass Cap

Well Status

Year Drilled: 2000

Total Depth

Start Date $9 / 18 / 00$

End Date $\quad 9 / 20 / 00$

Completion Depth: $41^{\prime}$

Easting:

Driller:DynatedRoy Brown Todays Date: 1/9/01

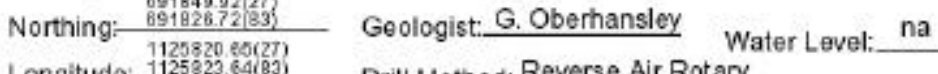

Latitude.

Mosuring Point

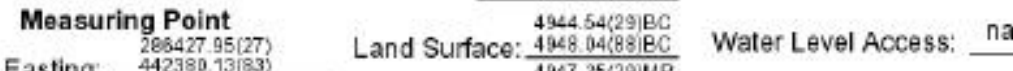

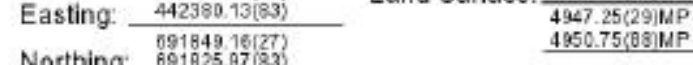

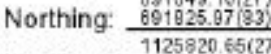

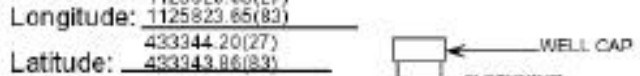

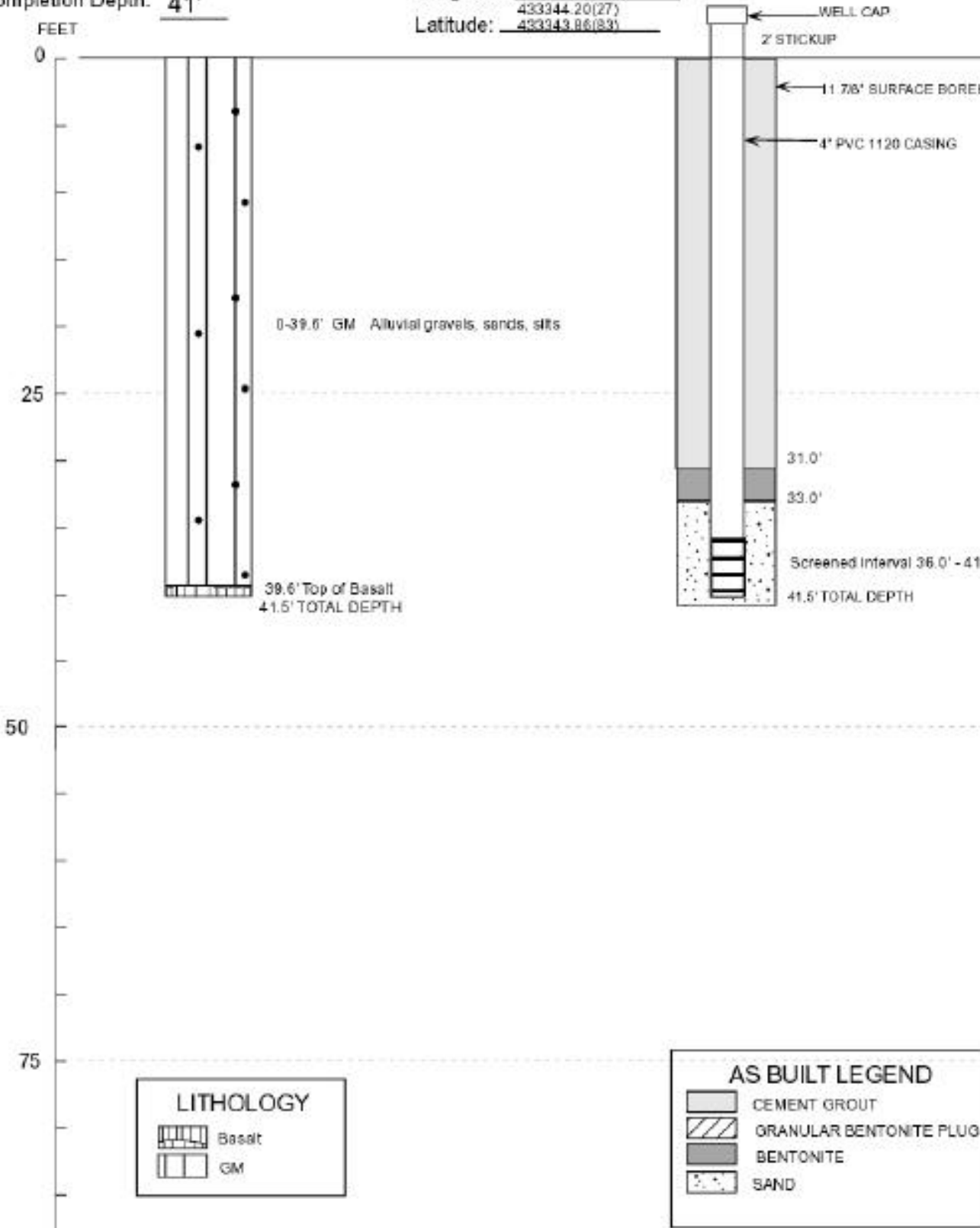

$$
\text { 피넴 Basolt }
$$

\begin{tabular}{|c|}
\hline AS BUILT LEGEND \\
\hline CEEEN GROUT \\
OMRNULAR BENTONTE PLUO \\
OENTONTE \\
SEND \\
\hline
\end{tabular}

100
Natural Gamma

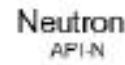

Gamma Gamma

$\underset{\substack{\text { Caliper } \\ \text { INCH }}}{\text { and }}$

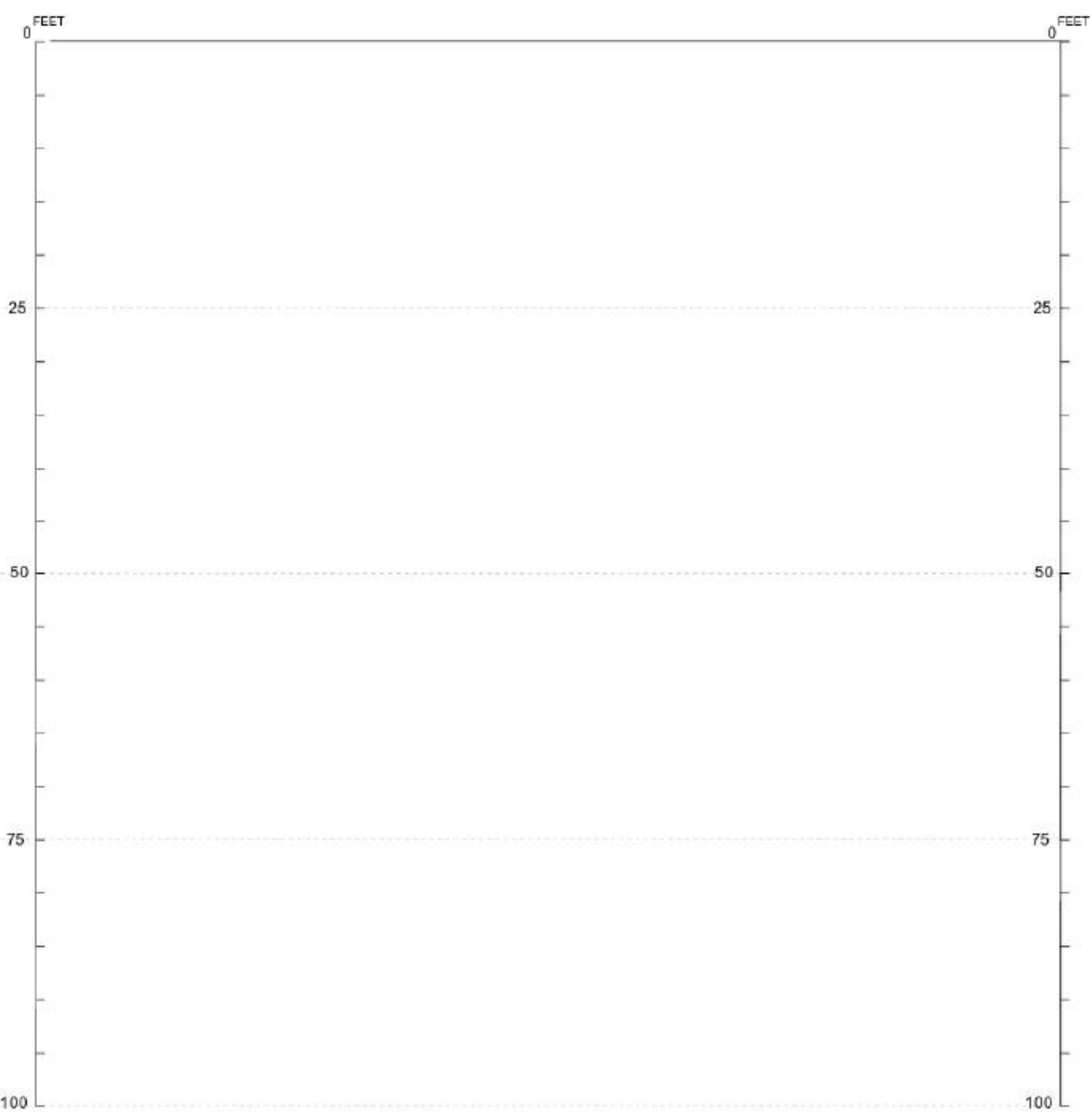


WELL NAME: ICPP-MON-V-191

Facility: INTEC (Perc Ponds)

Well Type: Monitoring

Well Status Active

Year Drilled: 2000

Total Depth $119^{\prime} 6^{\prime}$

Start Date $9 / 6 / 00$

Brass Cap

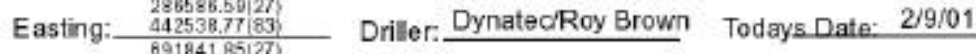

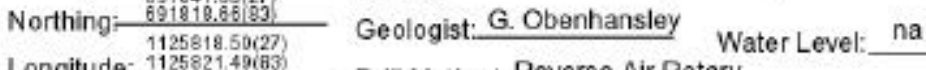

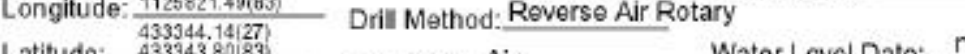
Dril Fluid:

End Date $\quad 9 / 7 / 00$

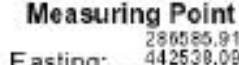

Water Level Access: na

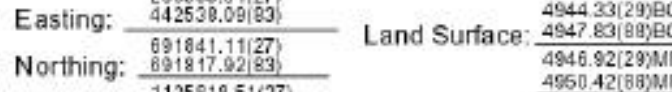

Complettion Depth: $118.75^{2}$

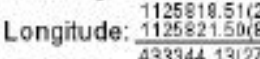

$$
\begin{array}{ll}
\text { FEET } \\
0
\end{array}
$$

PEet

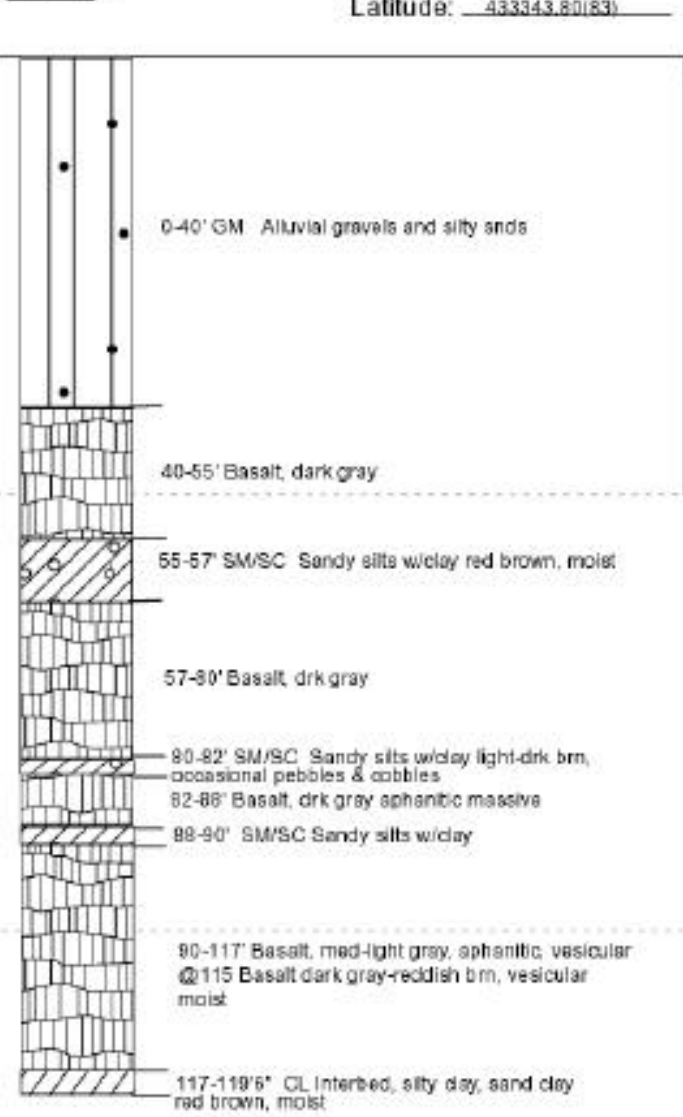

D飞walcap

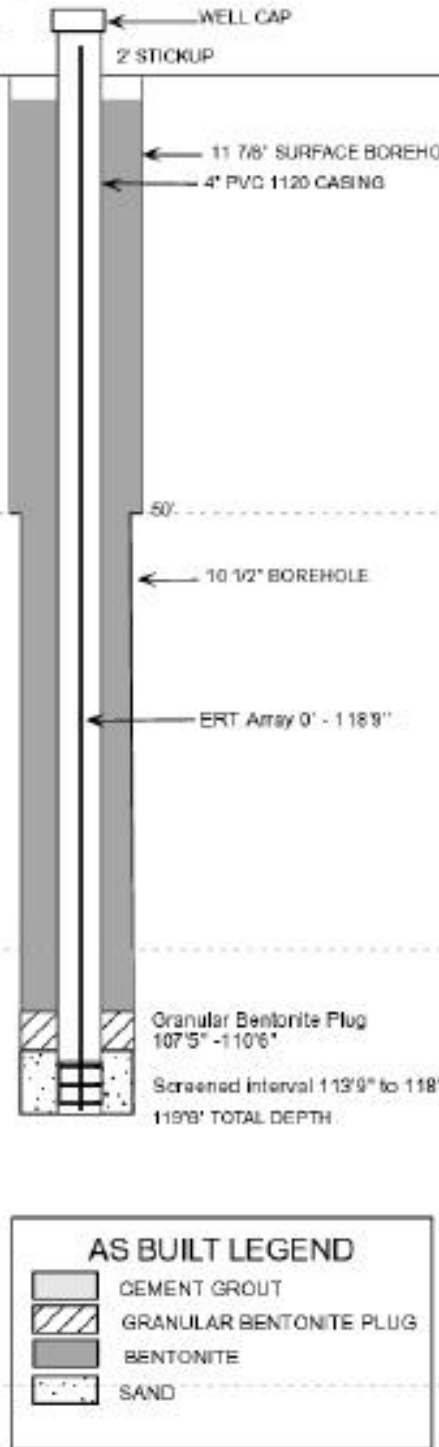

Natural Gamma

$\underset{\substack{\text { NePlN } \\ \text { Neutron }}}{\cos }$

$\underset{\text { GPS }}{\text { Gamma Gamma }}$

Caliper

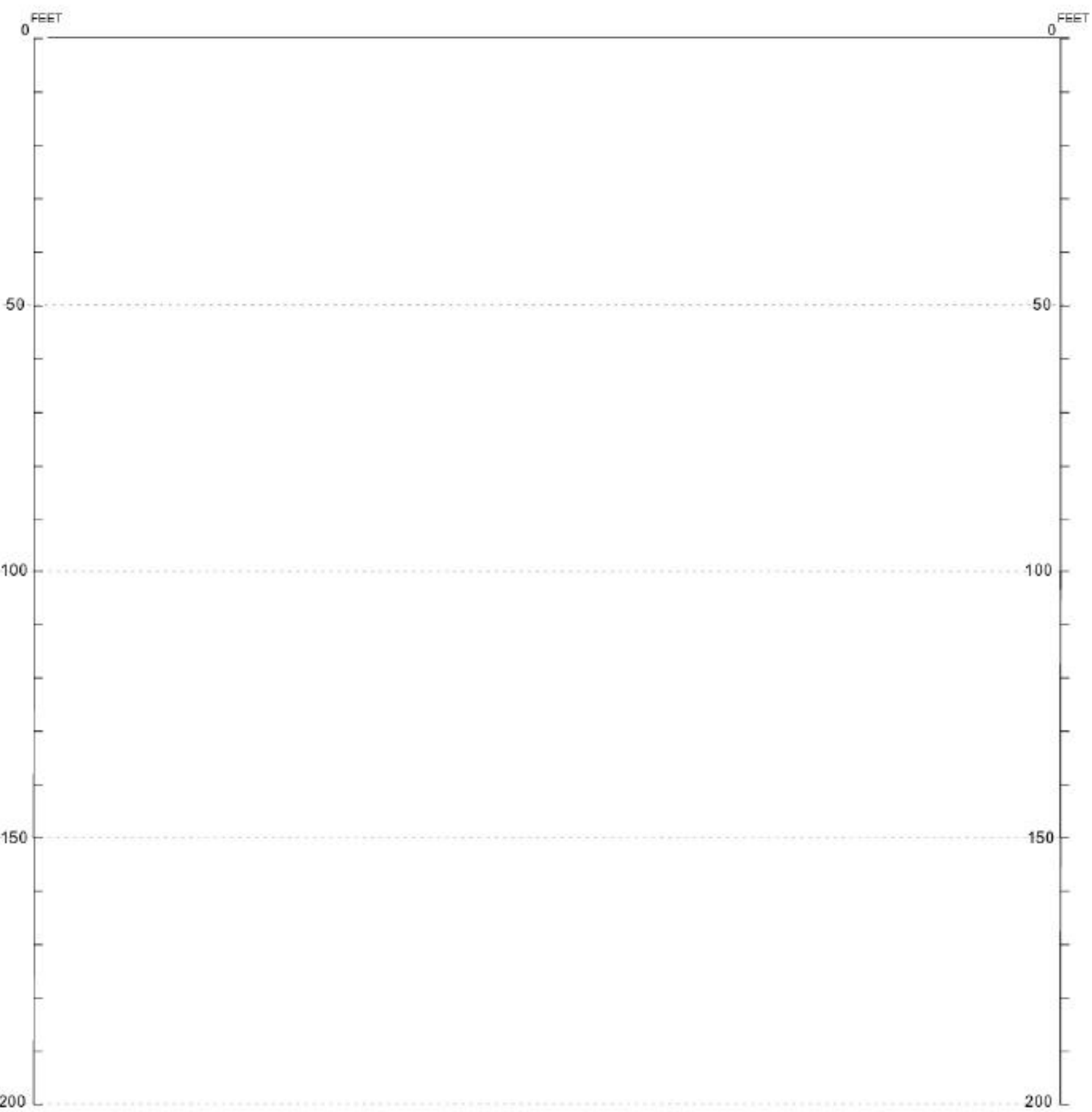


WELL NAME. ICPP.SCI-V-192 Facility: INTEC (Perc Ponds) Well Type: Instrumented Well Statustive

Year Drilled2001

Total Depth 157'

Start Date $1 / 10 / 0$

End Date $1 / 1 / 18 / 01$

mpletion Depth: 157

Brass Cap

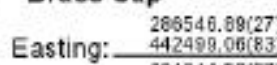

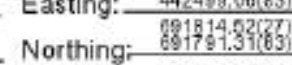

Driller: DynatedS. Tawater Todays Date: 6/21/01

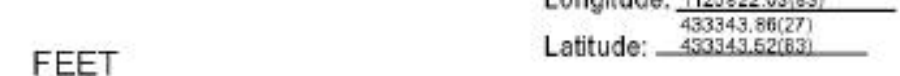
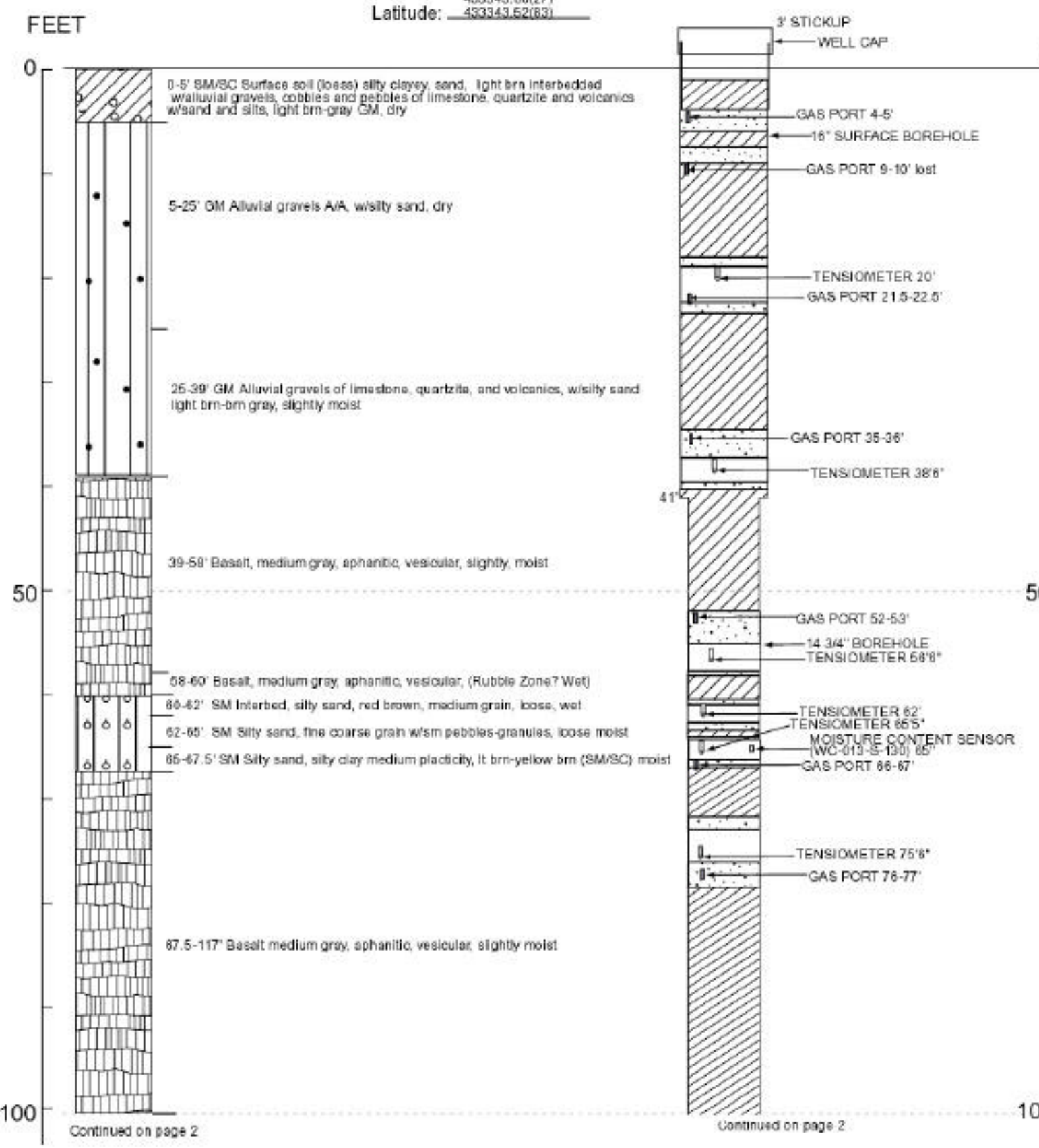

FEET

FEET。

$\circ$

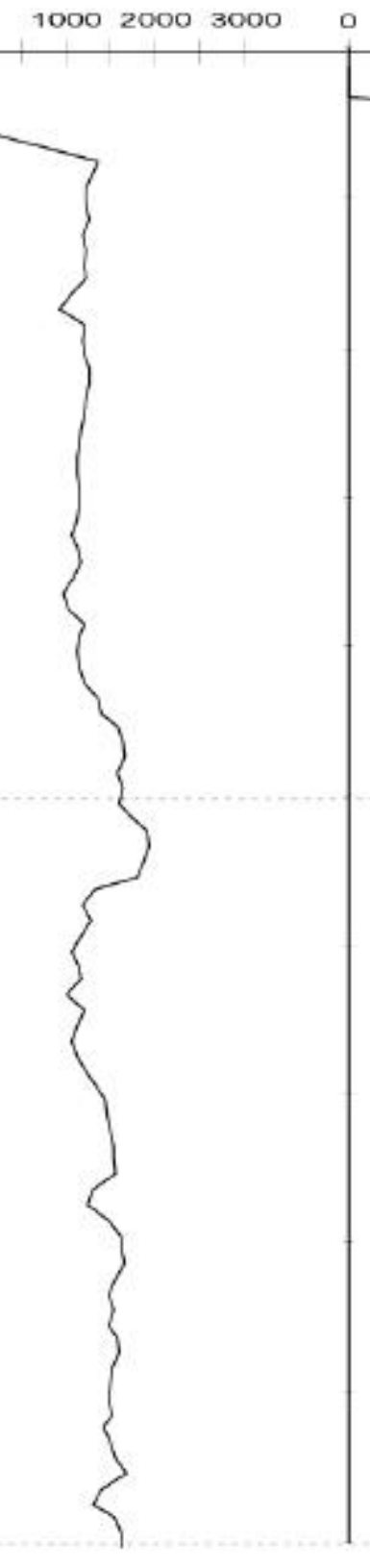

coninues on bose 2
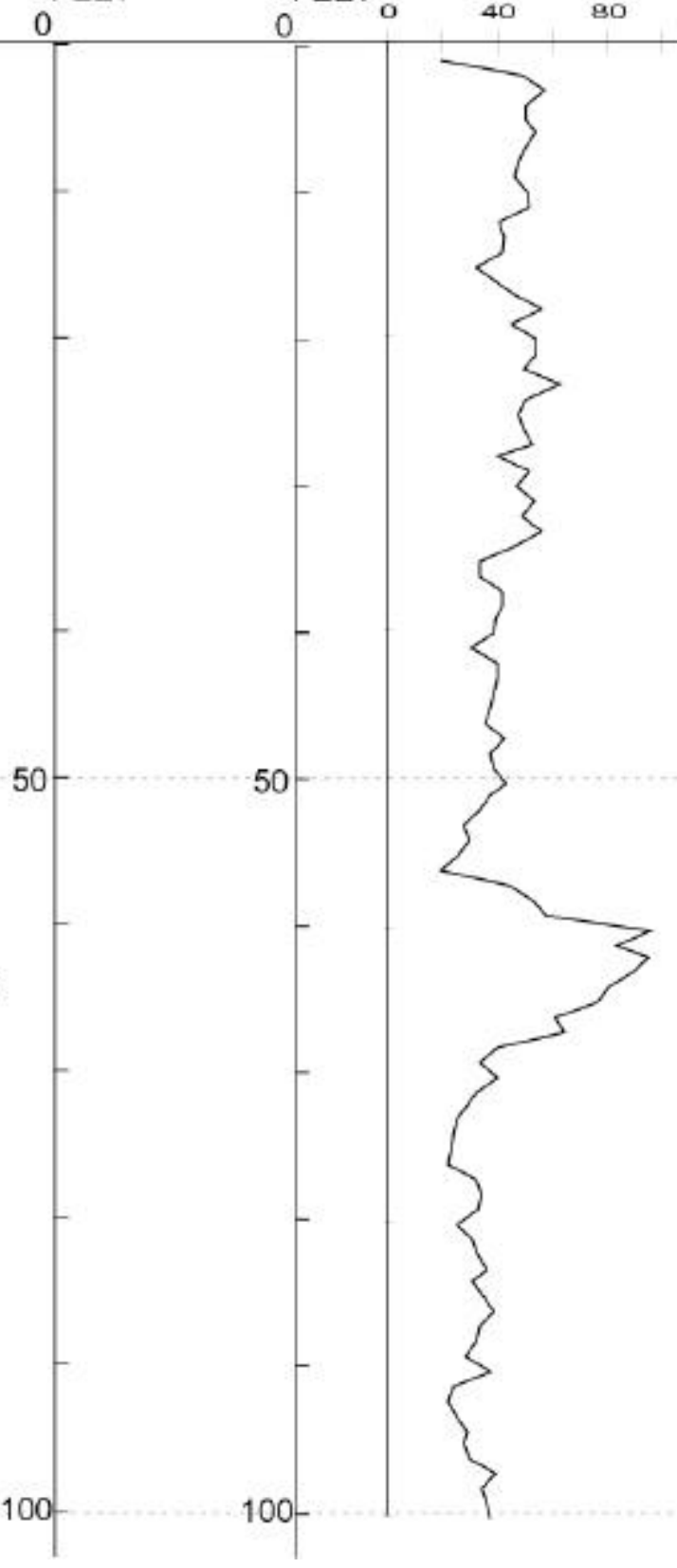

$\underset{C P S}{\operatorname{Gamma} G a m m a}$

Caliper

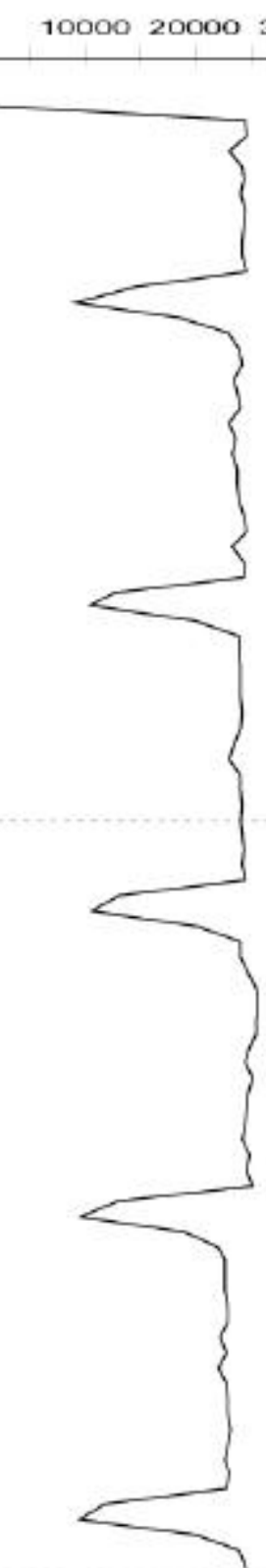

FEET

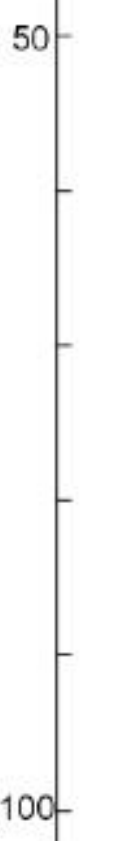


WELL NAME: ICPP-SCI-V-192 pg 2 Facility: INTEC (Perc Ponds) Instrumented

Well Statusctive

Year Drilled2001

Total Depth 157'

Start Date 1/10/01

End Date 1/12/01

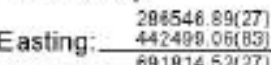

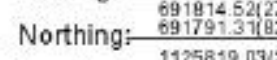

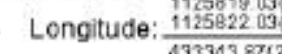

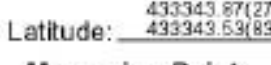

pletion Depth: $157^{\prime}$

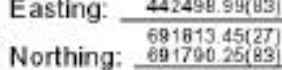

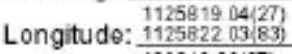

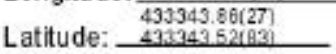

Driller: Dynated 1 . Tawater Todays Date: $5 / 1410$

Geologist:G. Oberhansley Water Level: na

Drill Method Reverse Air Rotary

Drill Fluid: Air

Water Level Access: na

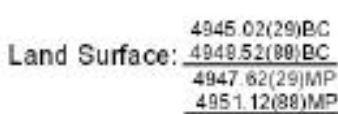

Contenued torm presenous poge Conninuec tom provivus osegs

Fentinueo tom provivus oges $\quad$ FEET

FEET

FEET 。

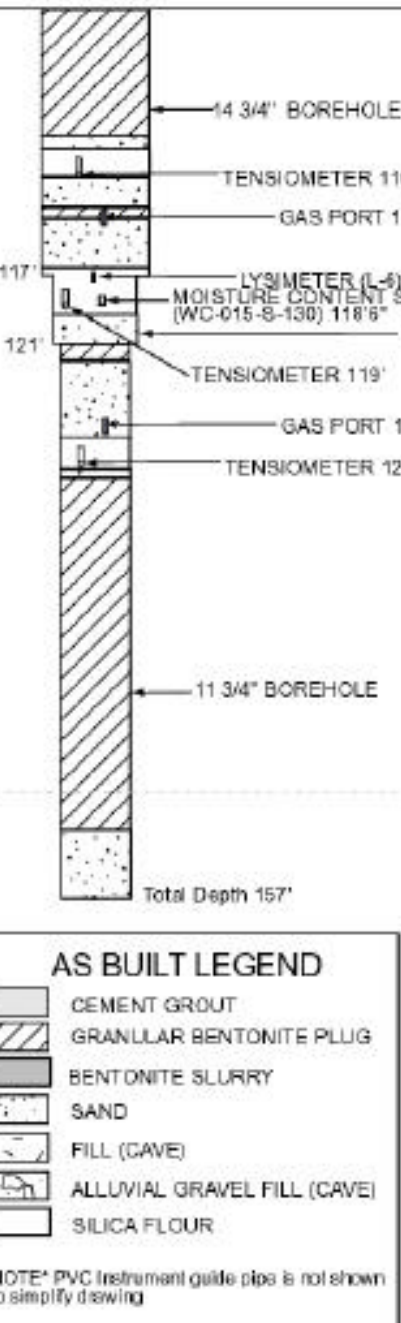

150

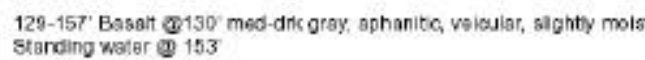

iㅔㅁㅔ

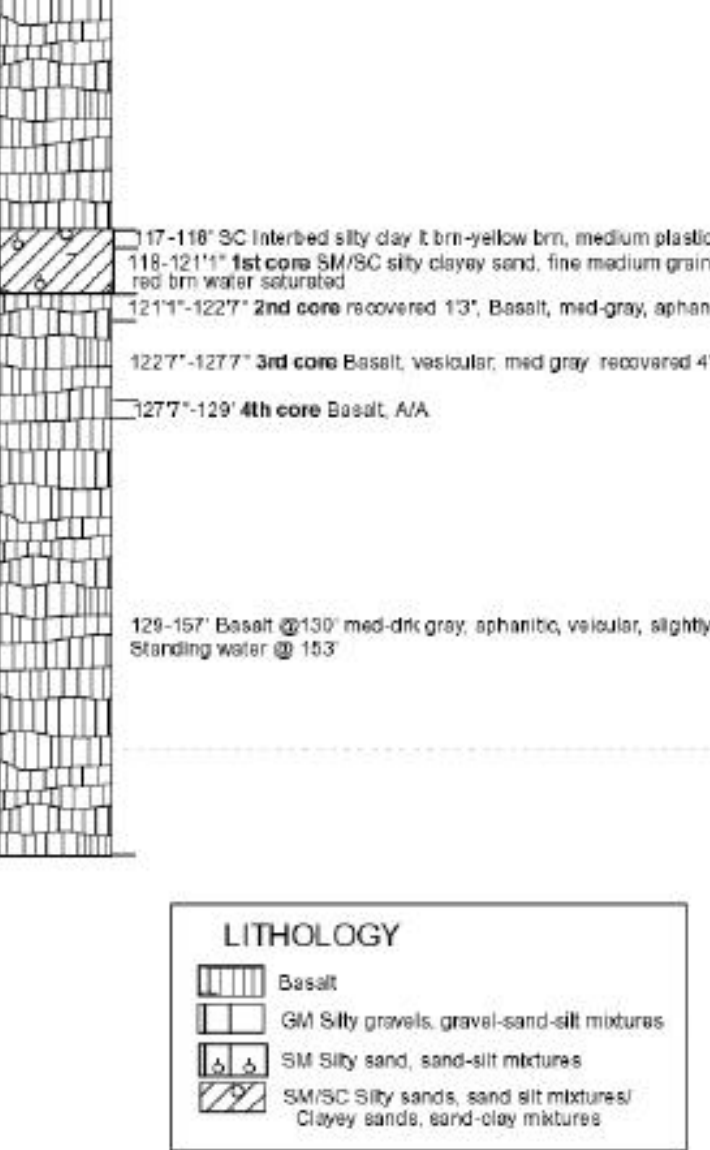

$200^{-}$

|

Natural Gamma

$\underset{\substack{\text { Neutron } \\ \text { AFI-N }}}{\cos }$

$\underset{\mathrm{CPS}}{\text { Gamma Gamma }}$

$\underset{\substack{\text { Caliper } \\ \text { INCH }}}{\text { (n) }}$

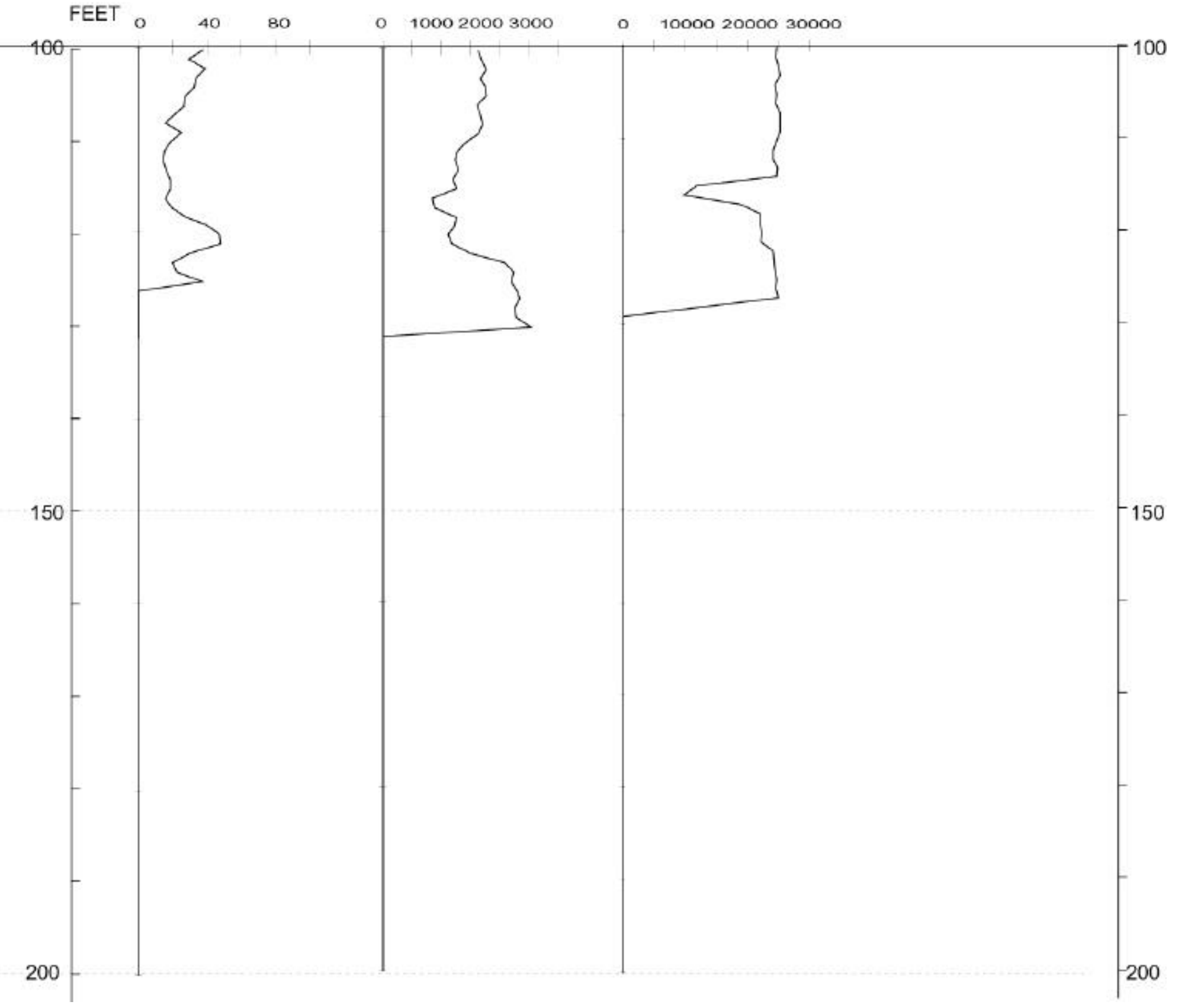




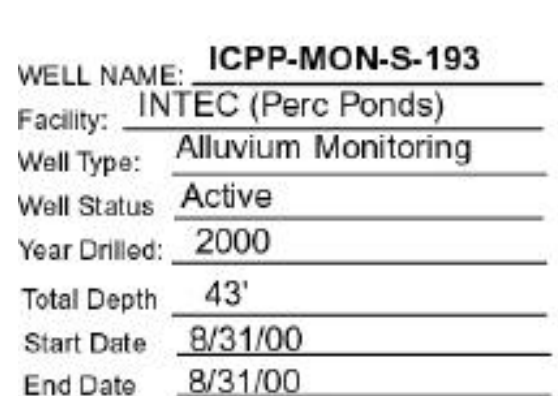

Completion Depth: 40.3

FEET$$
\text { FEET }
$$

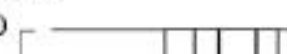

Brass Cap

Easting: $\frac{286529.442}{462456.629}$

Diller.DynatedRoy Brown Todays Date: 1/9/01

Geologist:T. MatsonKK. Dooley Water Level: na

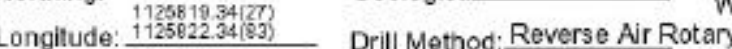

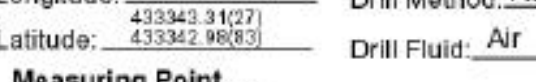

Water Level Date: na

Measuring Point

Water Level Access: na

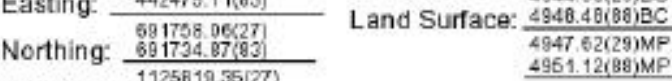

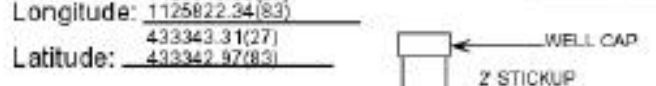
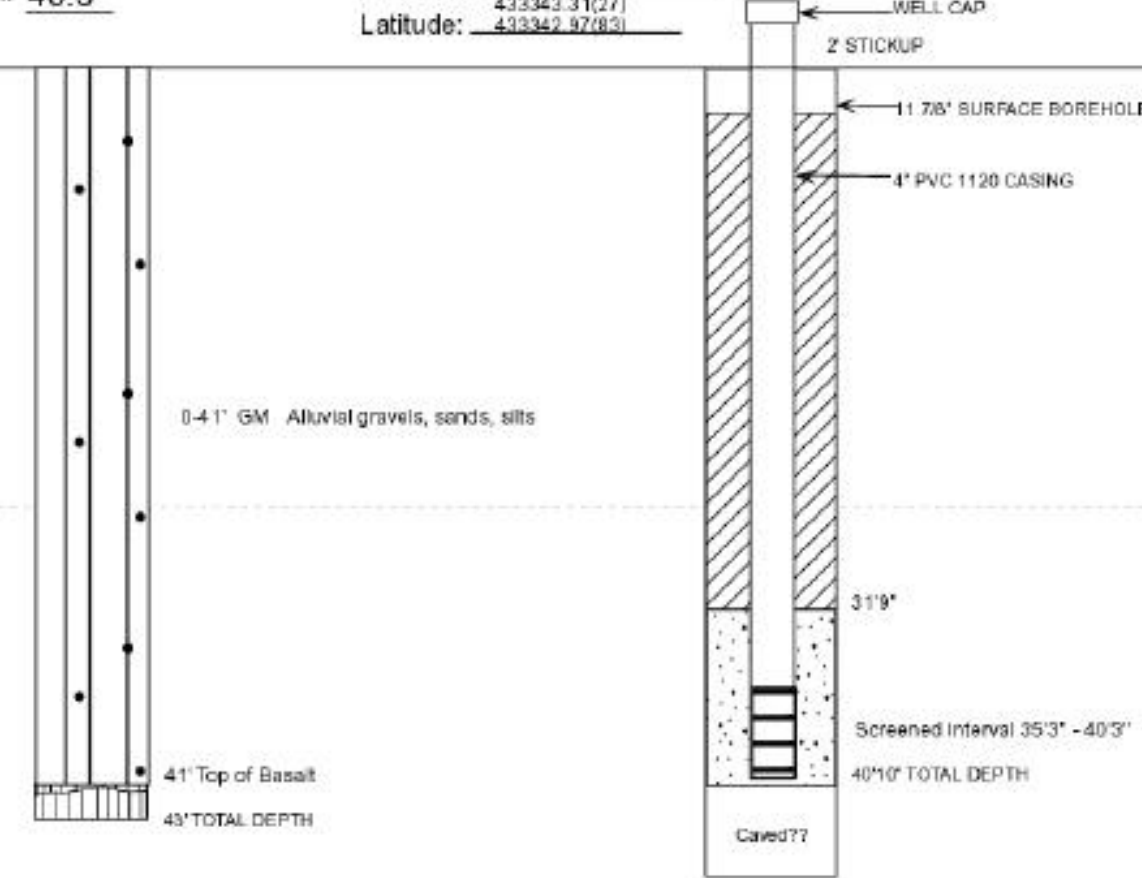

LITHOLOGY

피밀 Basat

$100 \mathrm{~L}$

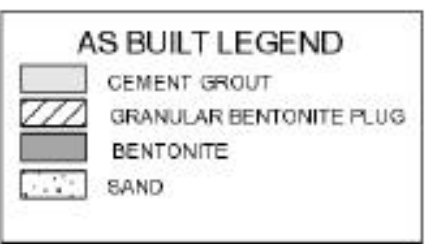

Z2 GRANULAR BE

BENTON
BANO
Natural Gamma

$\underset{\substack{\text { Neutron } \\ \text { APIN }}}{\cos }$

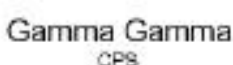

$\underset{\substack{\text { Caliper } \\ \text { Cat }}}{\text { Con }}$

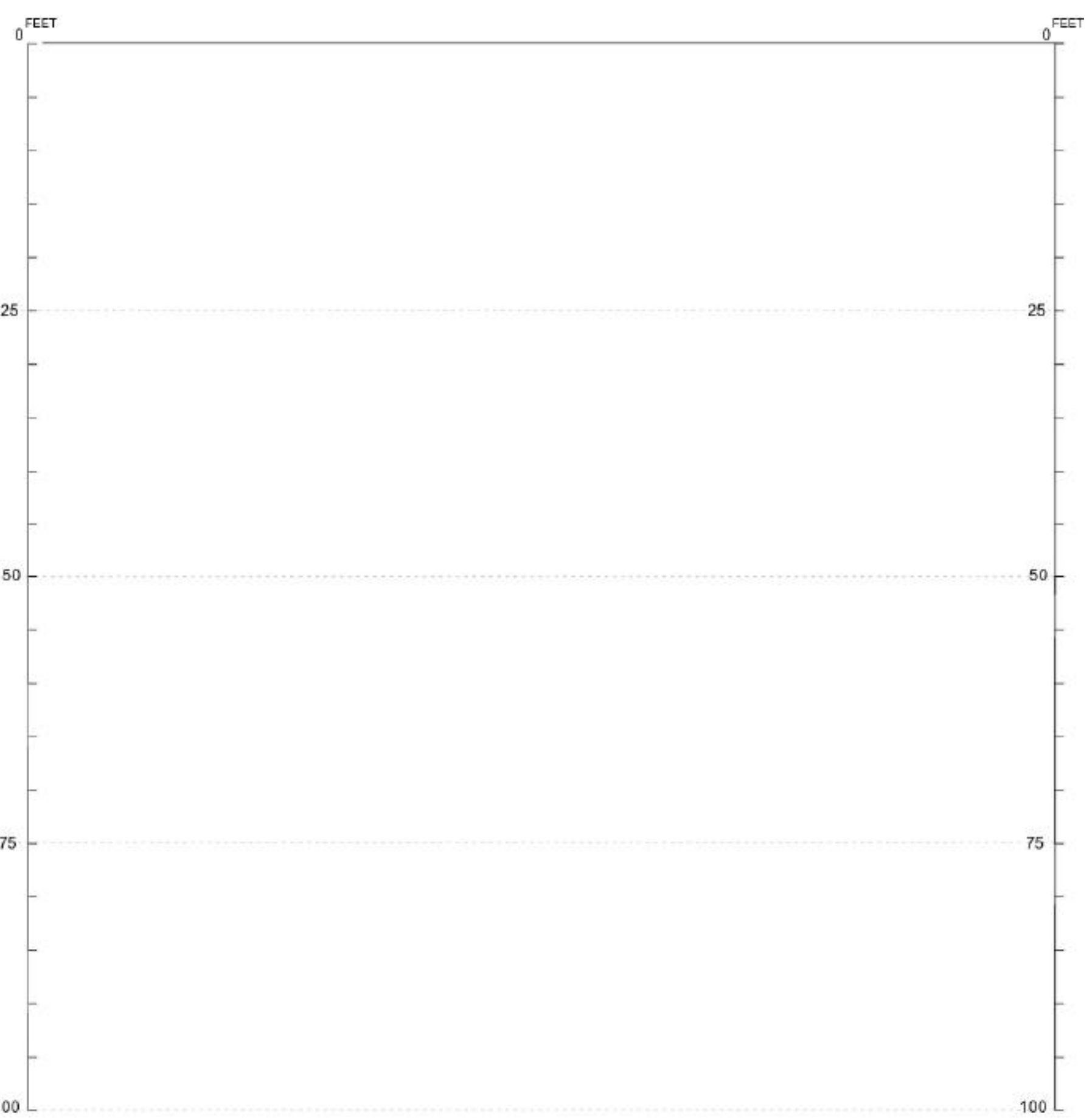




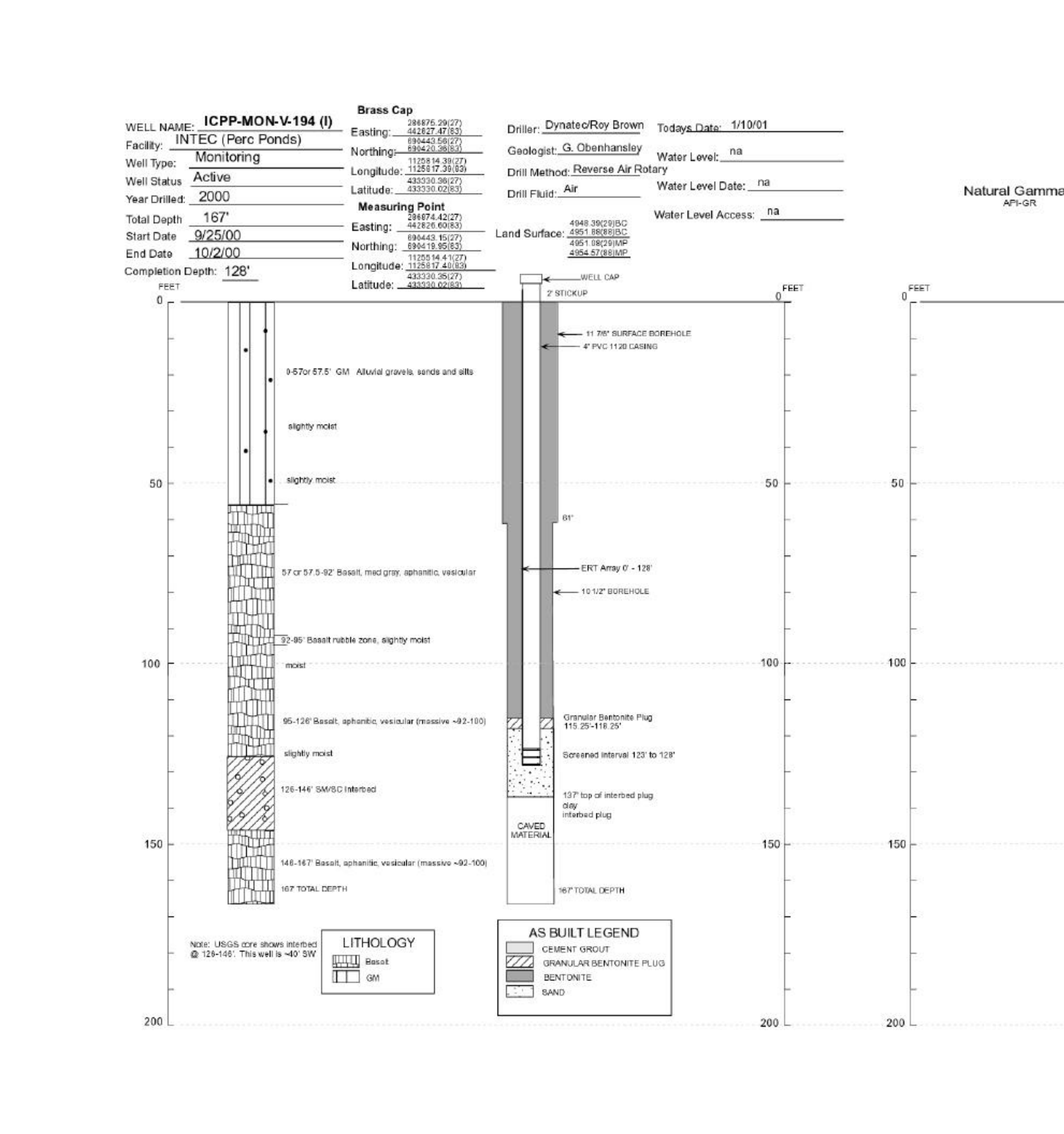




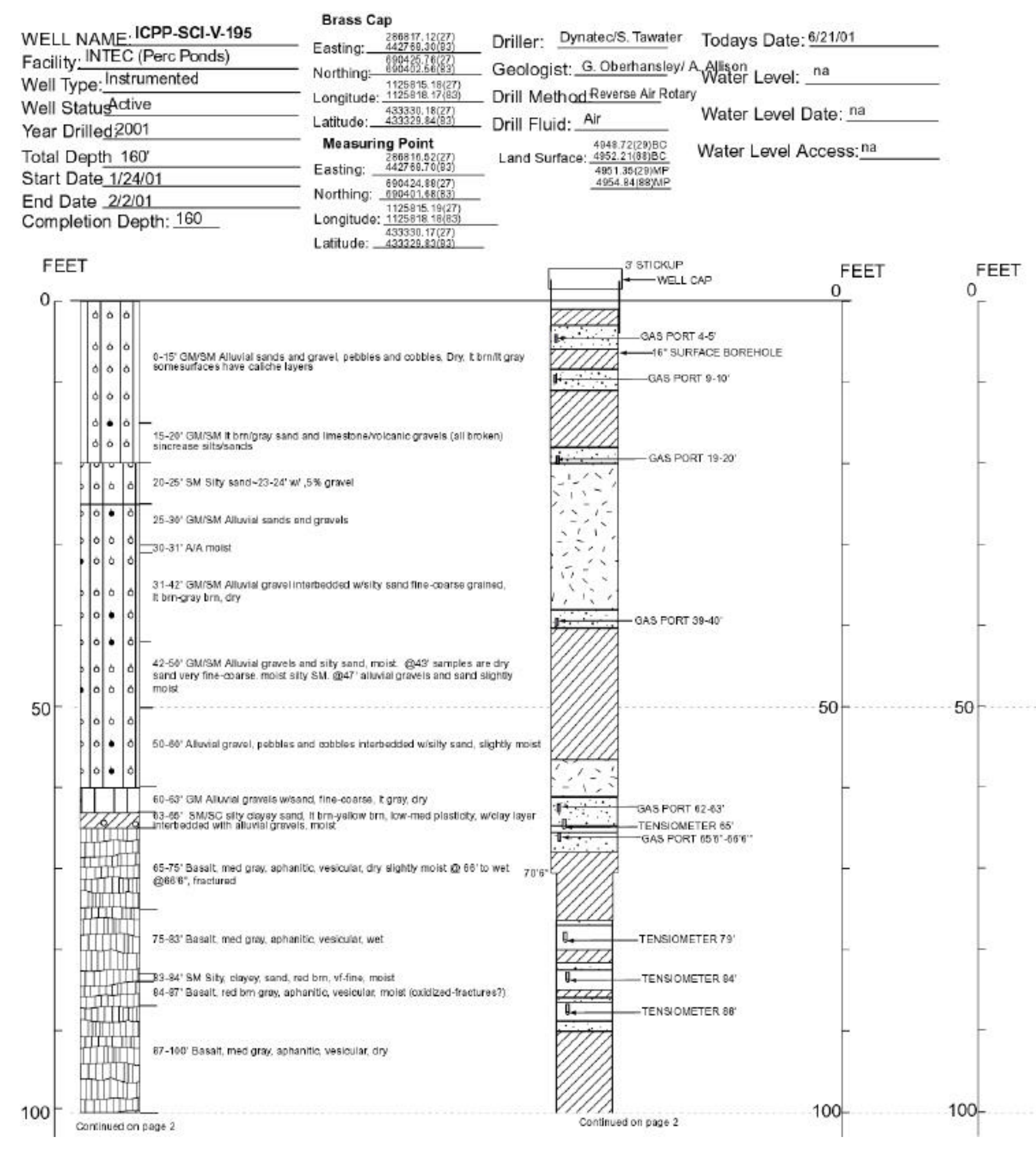

(


WELL NAME. ICPP.SCI-V-195 pg 2
Facility: INTEC (Perc Ponds)

Well Status Active

Year Drilled

Total Depth $160^{\prime}$

Start Date $1 / 24,01$

Completion Depth: $160^{\circ}$

\section{Brass Cap}

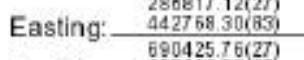

Diller: Dynatecis. Tawater Todays Date:6/2101

G. Obenansley Water Level: na

Northing Reverse Air Row Water Level.

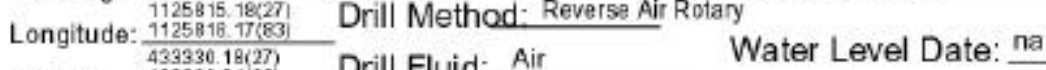

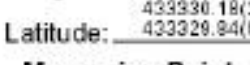

Drill Fluid: Air

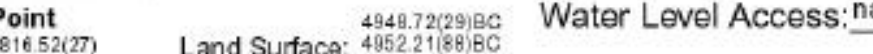

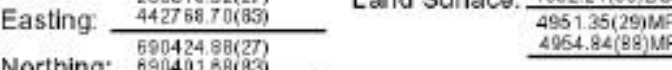

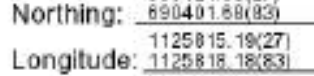

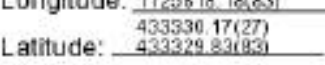

Continued from prevous page

FEET

FEET

Natural Gamma

$\underset{\substack{n \\ A P I+N}}{\text { Neutron }}$

Gamma Gamma

$\underset{\substack{\text { Caliper } \\ \text { INCH }}}{\cos }$

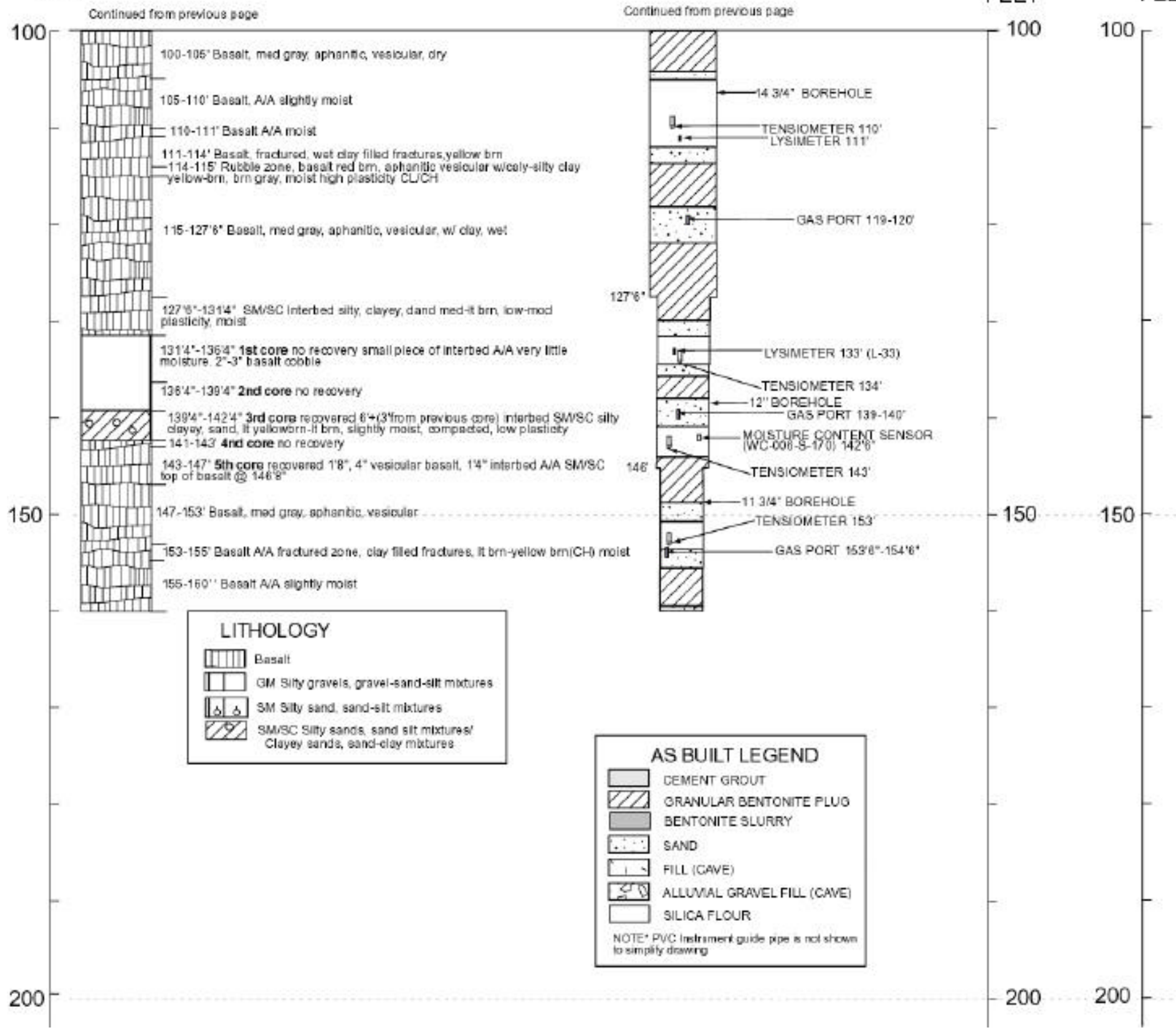



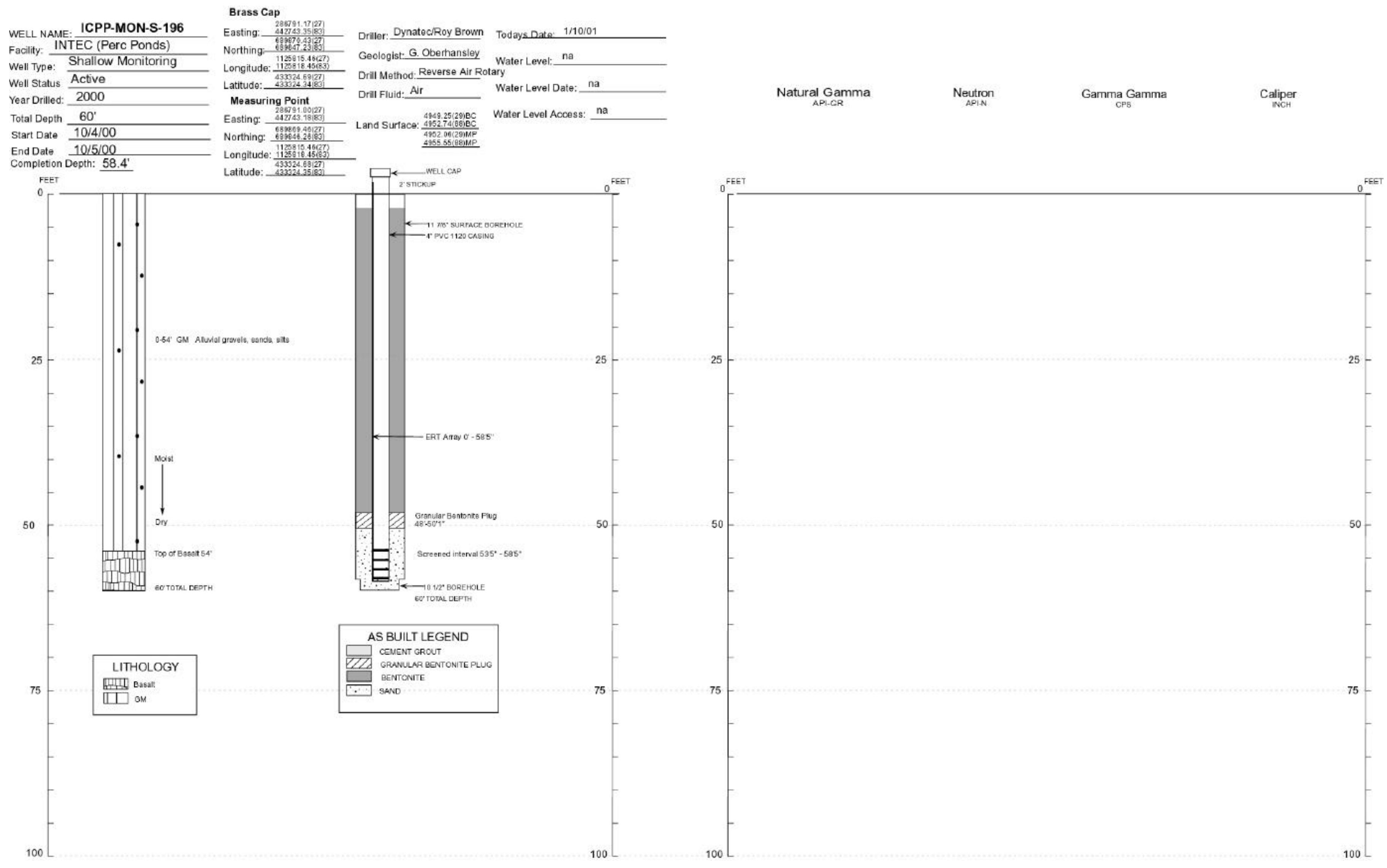

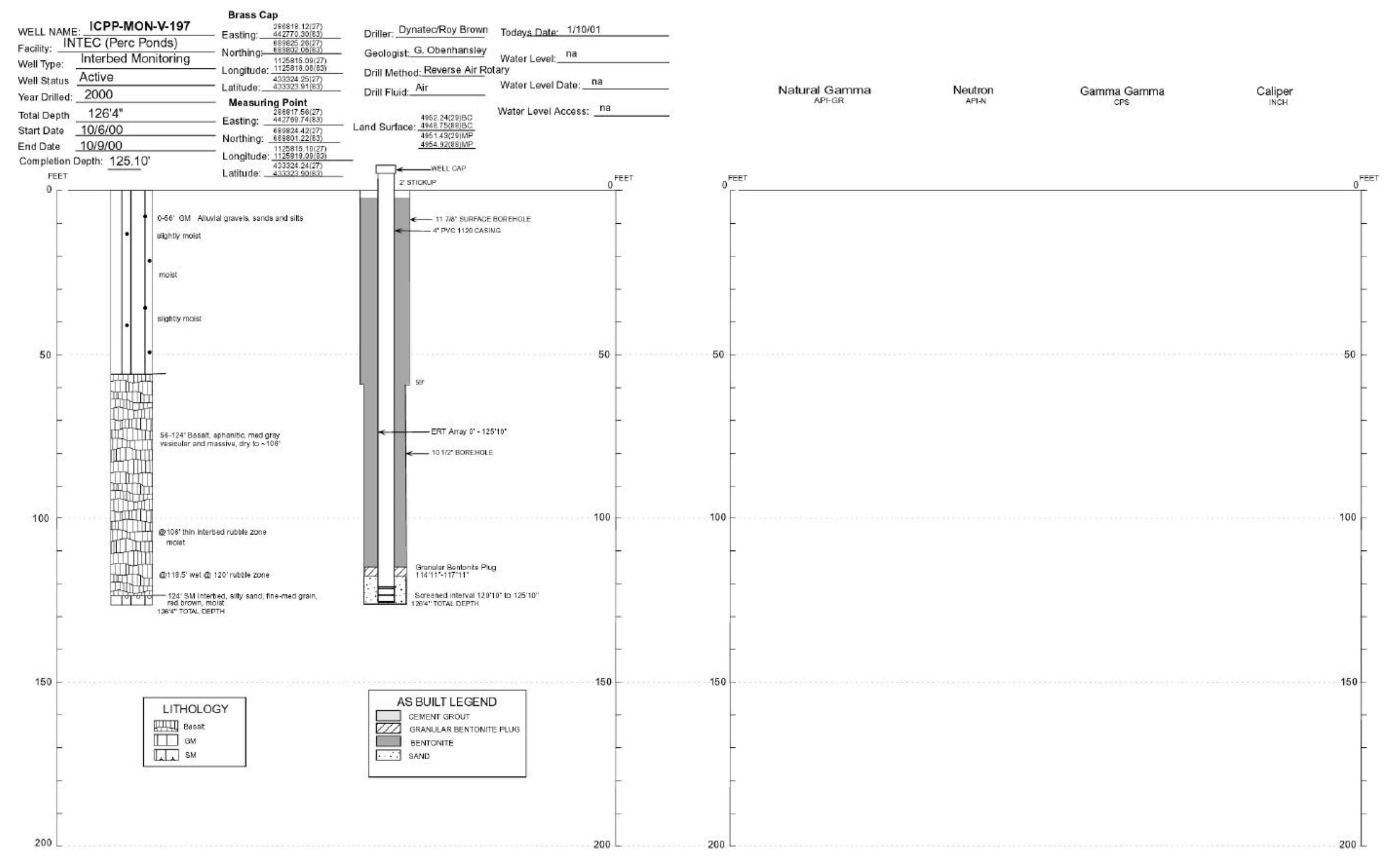


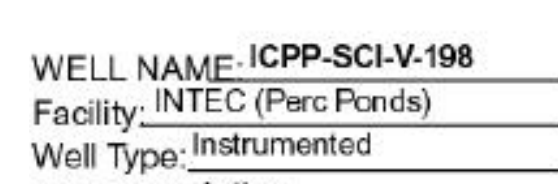

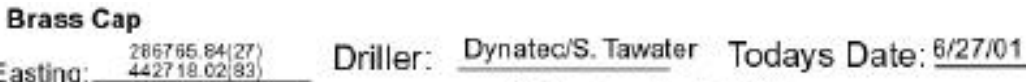

Instrumented

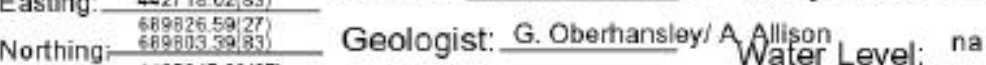

Well Statusctive

Year Drilled2001

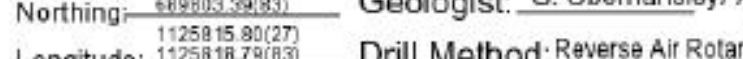

Total Depth 159' $\frac{2332425[27)}{427}$ Dil Mater Level Date:

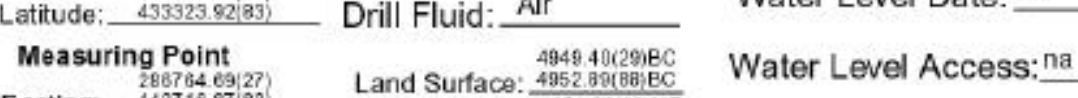

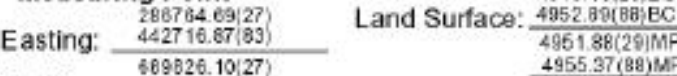

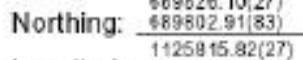

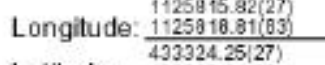

Completion Depth: 159

Latitude:

FEET

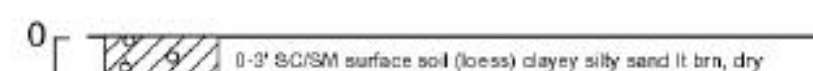

${ }^{0}$.

$-$

50

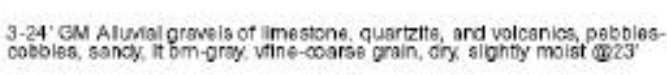

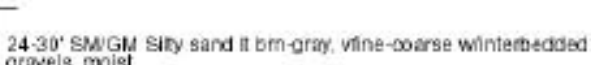

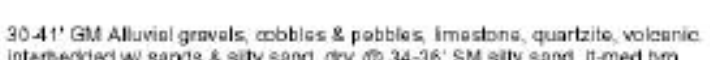

10.10 . mena

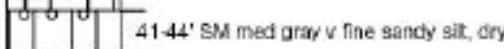

1000

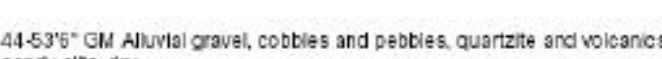

$100-$

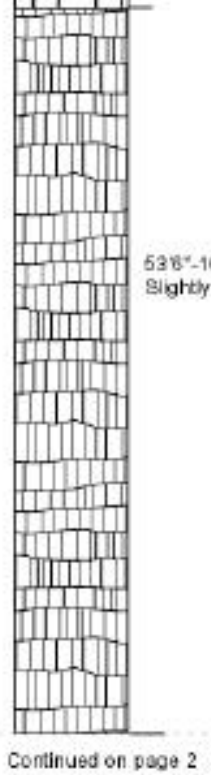

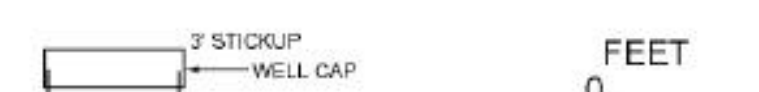

FEET

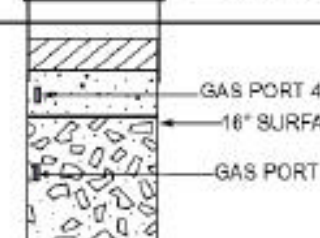

GAS PORT 19.20

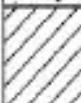

100 :

in

I:. GAS PORT 39-40

एणं

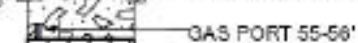

DT 14 34: BOREHOLE

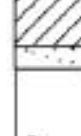

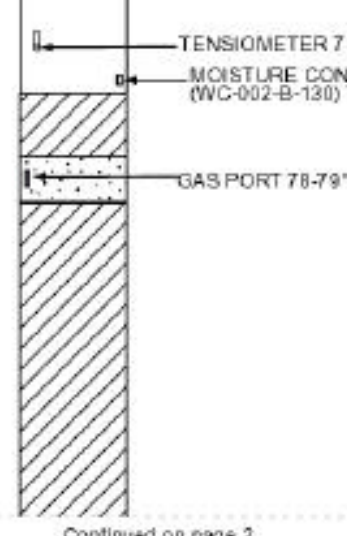

OAS PORT 50.5T.

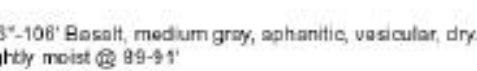

Natural Gamma

$\underset{\text { AFI-N }}{\text { Neutron }}$

$\underset{\text { CPS }}{\text { Gamma Gamma }}$

$\underset{\substack{\text { Caliper } \\ \text { INCH }}}{\text { and }}$

FEET 。

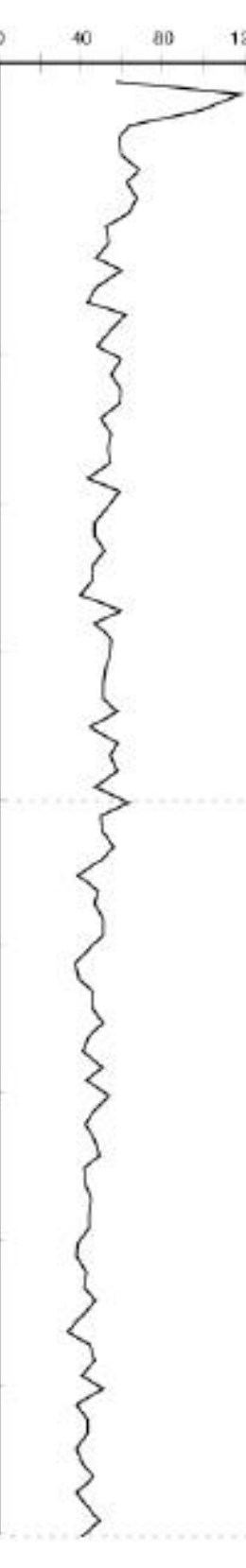
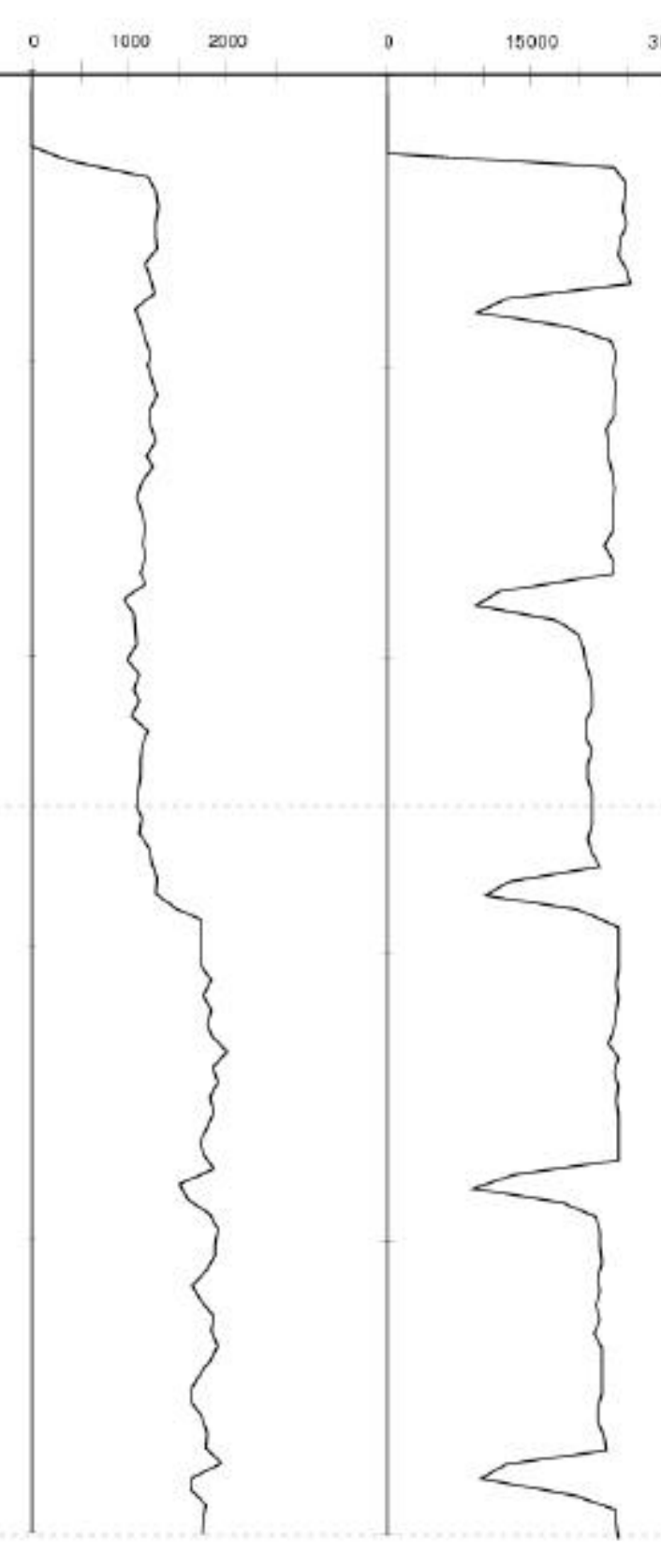

FEE

$50-$

50

100

100 30000 


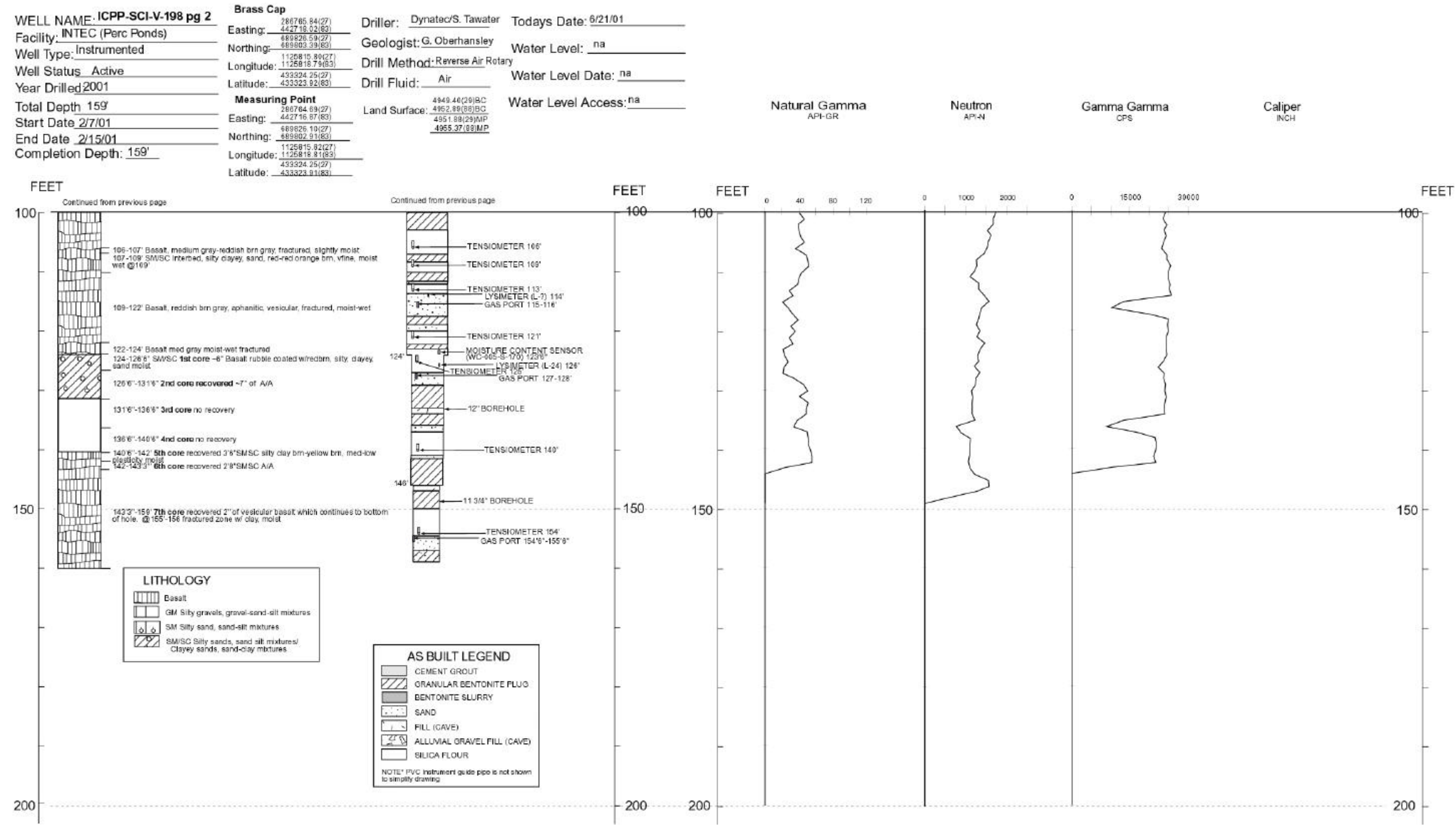


WELL NAME: ICPP-MON-S-199

Facility:
Well Type: Shallow Monitoring

Brass Cap

Well Status Active

Year Drilled 2000

Total Depth $51.58^{\prime}$

Start Date $\frac{51.58}{10 / 17 / 00}$

Water Level: na

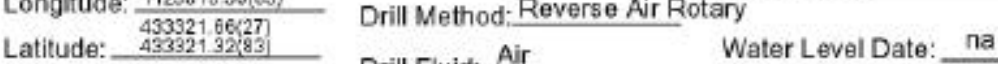

Me asuring Point

Completion Depth: 51

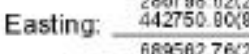

Water Level Accoss: na$$
\text { FEE }
$$

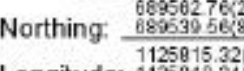

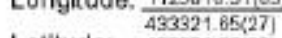$$
{ }^{0}
$$
(1)

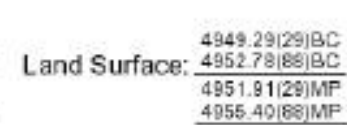

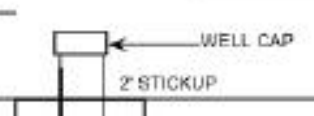

50

75

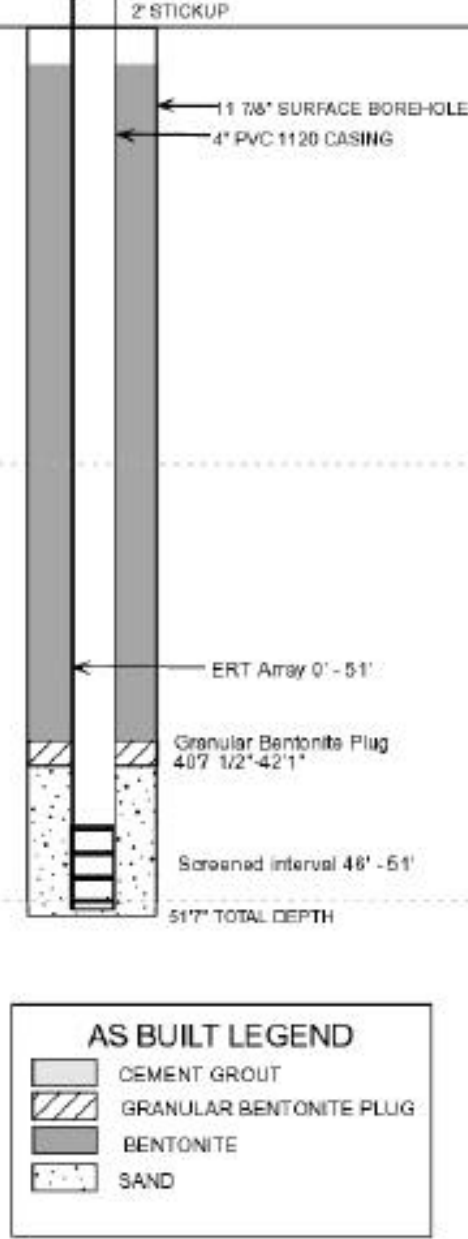

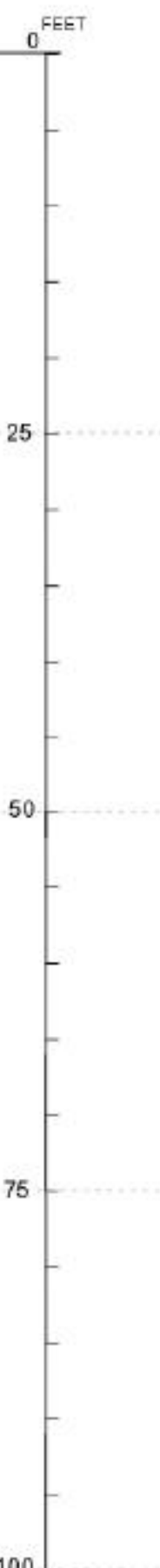

Natural Gamma

Neutron

Gamma Gamma

Caliper

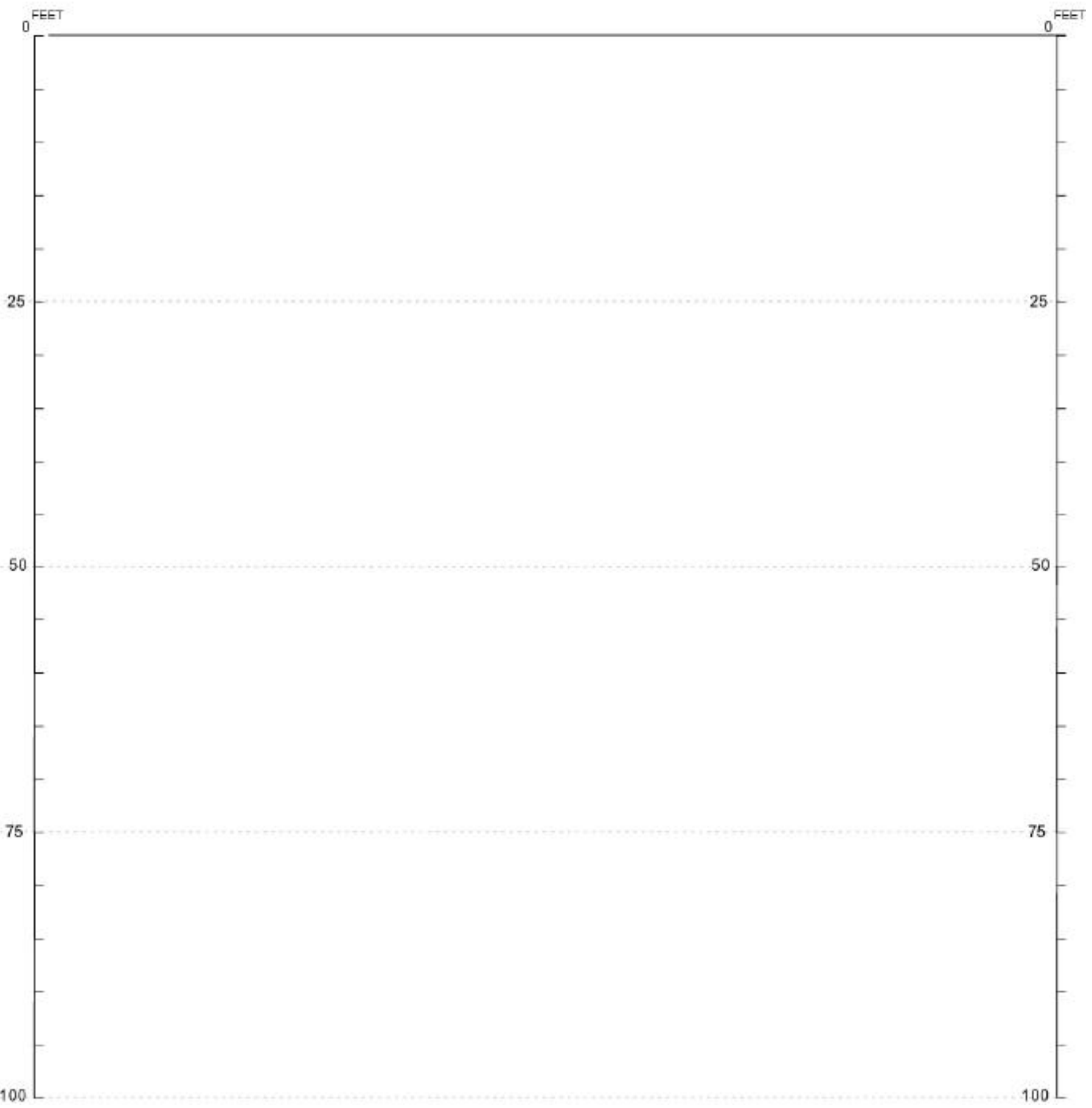




$$
\text { Int }
$$


Brass Cap

\begin{tabular}{|c|c|c|c|}
\hline \multirow{2}{*}{ WELL NAME: ICPP-SCI-V-201 } & \multirow{2}{*}{$\begin{array}{l}\text { Easting: Not surveyed } \\
\text { Northing: }\end{array}$} & \multirow{2}{*}{$\begin{array}{l}\text { DynateciS. Tawater } \\
\text { Driller: } \frac{\text { /6. Jensen }}{}\end{array}$} & \multirow{3}{*}{$\begin{array}{l}\text { Todays Date: 8/8/01 } \\
\text { Watar Level: } \mathrm{na}\end{array}$} \\
\hline & & & \\
\hline Well Type: Instrumented Monitoring & Longitude: & & \\
\hline Well Status Abandoned & Latitude:- & Drill Method:Reverse Air Rota & $y$ \\
\hline Year Drilled: 2001 & Measuring Point & Drill Fluid: Afr & \\
\hline Total Depth $\quad 262$ & Easting. _- & Land Surface: & Water Level Acosss: na \\
\hline Start Date $5 / 2 / 01$ & Northing: & & \\
\hline $\begin{array}{l}\text { End Date } \\
\text { Completion Depth: } 247^{\prime} 8^{\prime \prime}\end{array}$ & Longitude: & & \\
\hline
\end{tabular}
$\underset{\substack{\text { Natural Gamma } \\ \text { AP.GR }}}{ }$
$\underset{\substack{\text { Neutron } \\ \text { APPN }}}{\text { nats }}$
$\underset{\mathrm{CPS}}{\text { Gamma Gamma }}$
$\underset{\substack{\text { Caliper } \\ \text { INCH }}}{\text { and }}$

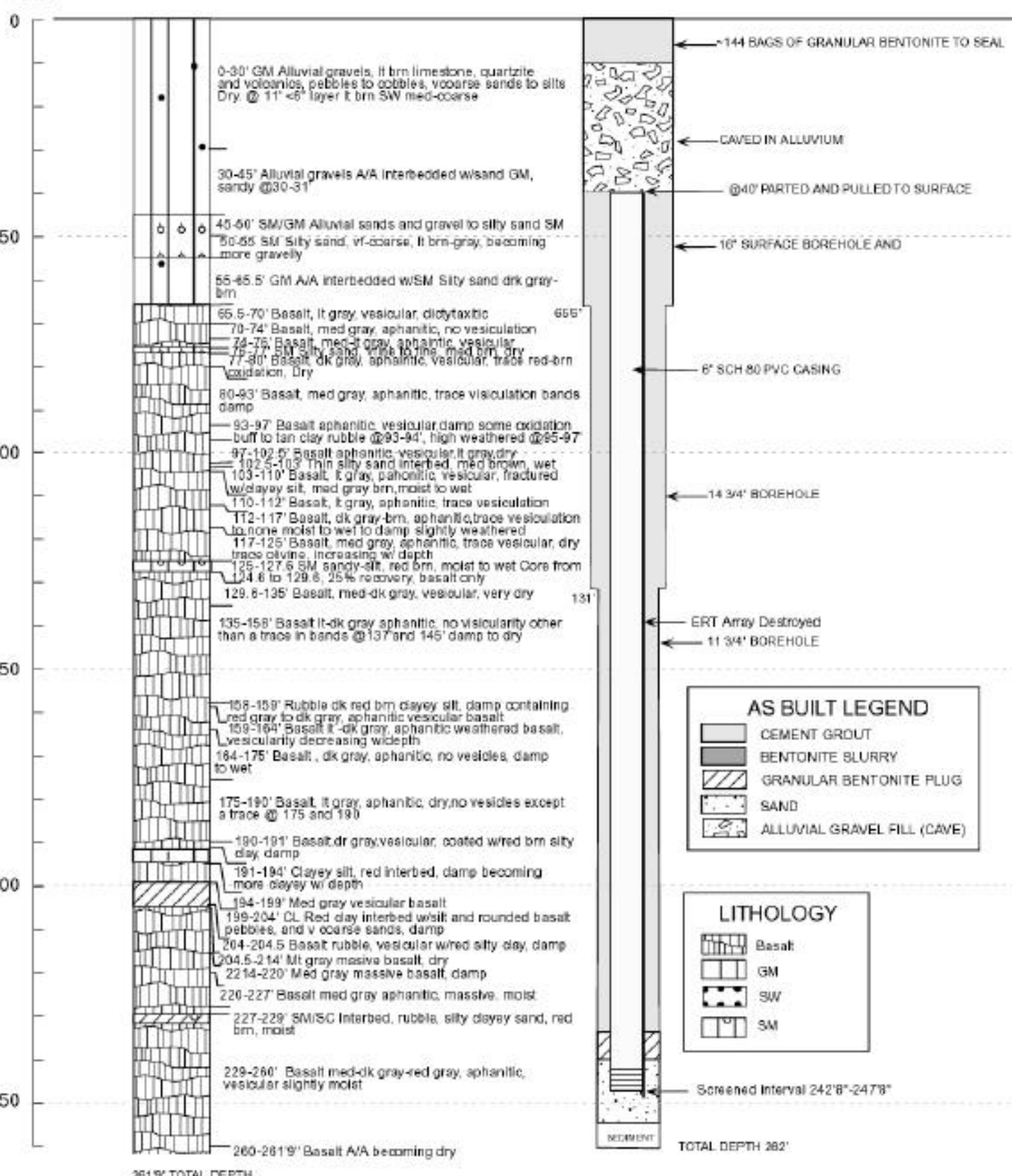

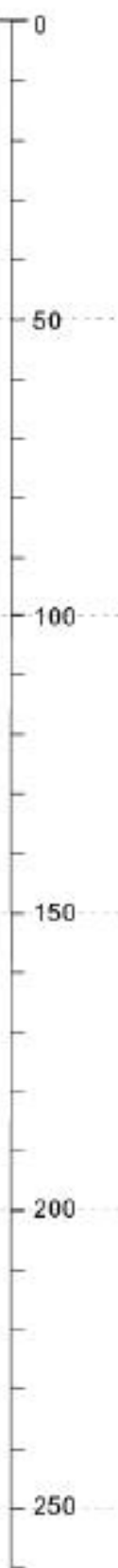

$-250$

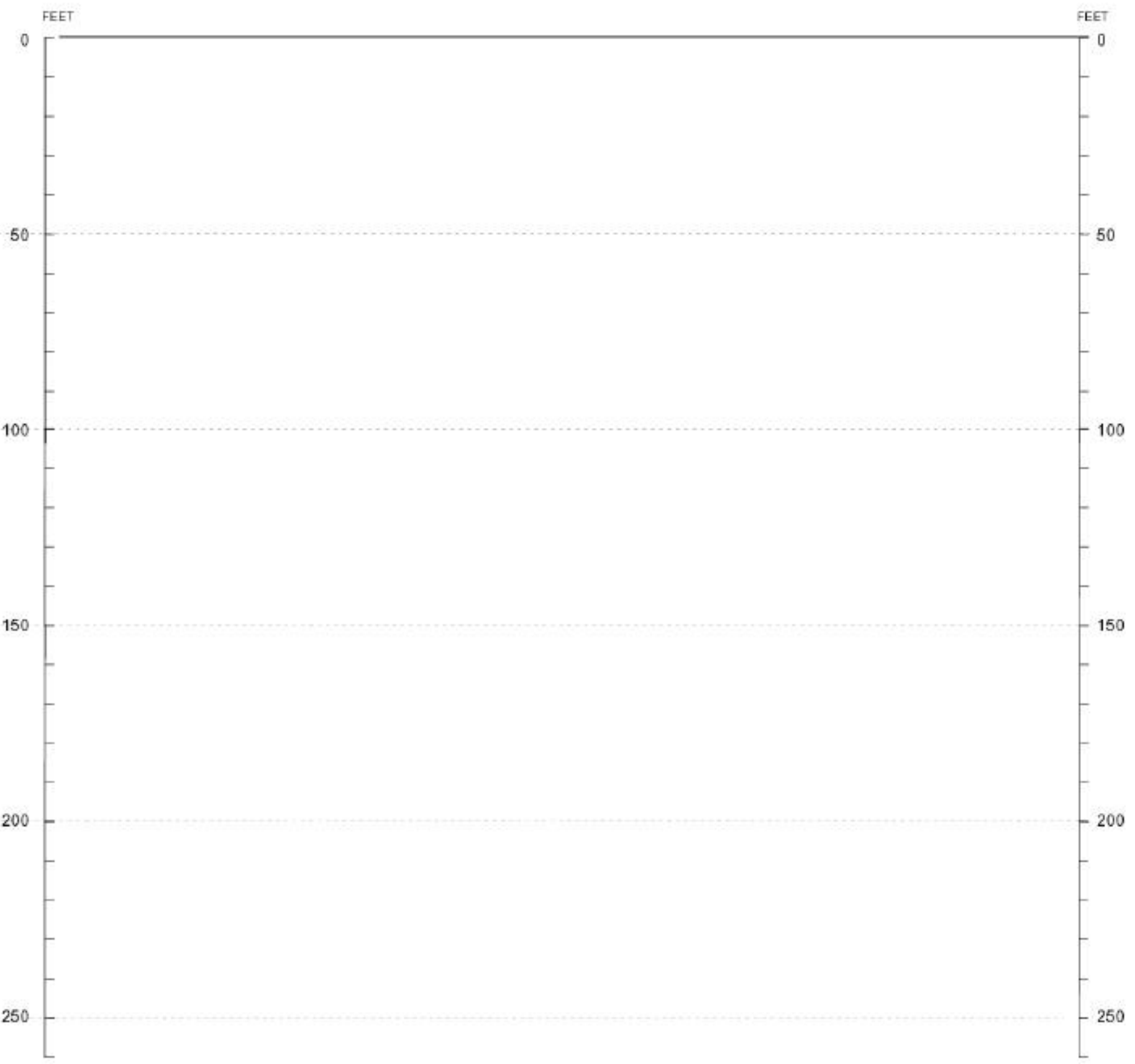


WELL NAME: ICPP-SCI-V-201A

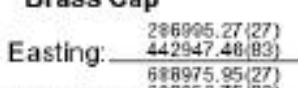

WELL NAME: CPP-SCI-V-201A

Instrumented Monitoring Lo

Well Status Active

Year Drilled: 2001

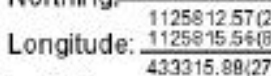

Driller: DynatedG. Jensen

Goologist G. Obenhansley

Drill Methood: Reverse Air Rotary

Latitude: 433315.54:5i

Drill Fluid: Air

Total Depth 262'

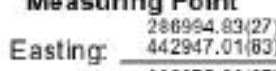

Drill Fluid:A 4848.8129180

Water Level Date:

Start Date

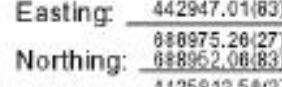

End Date 6/29/01

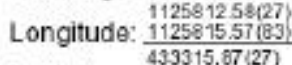

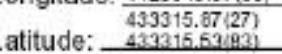

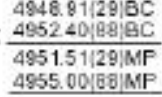

Water Level Access:

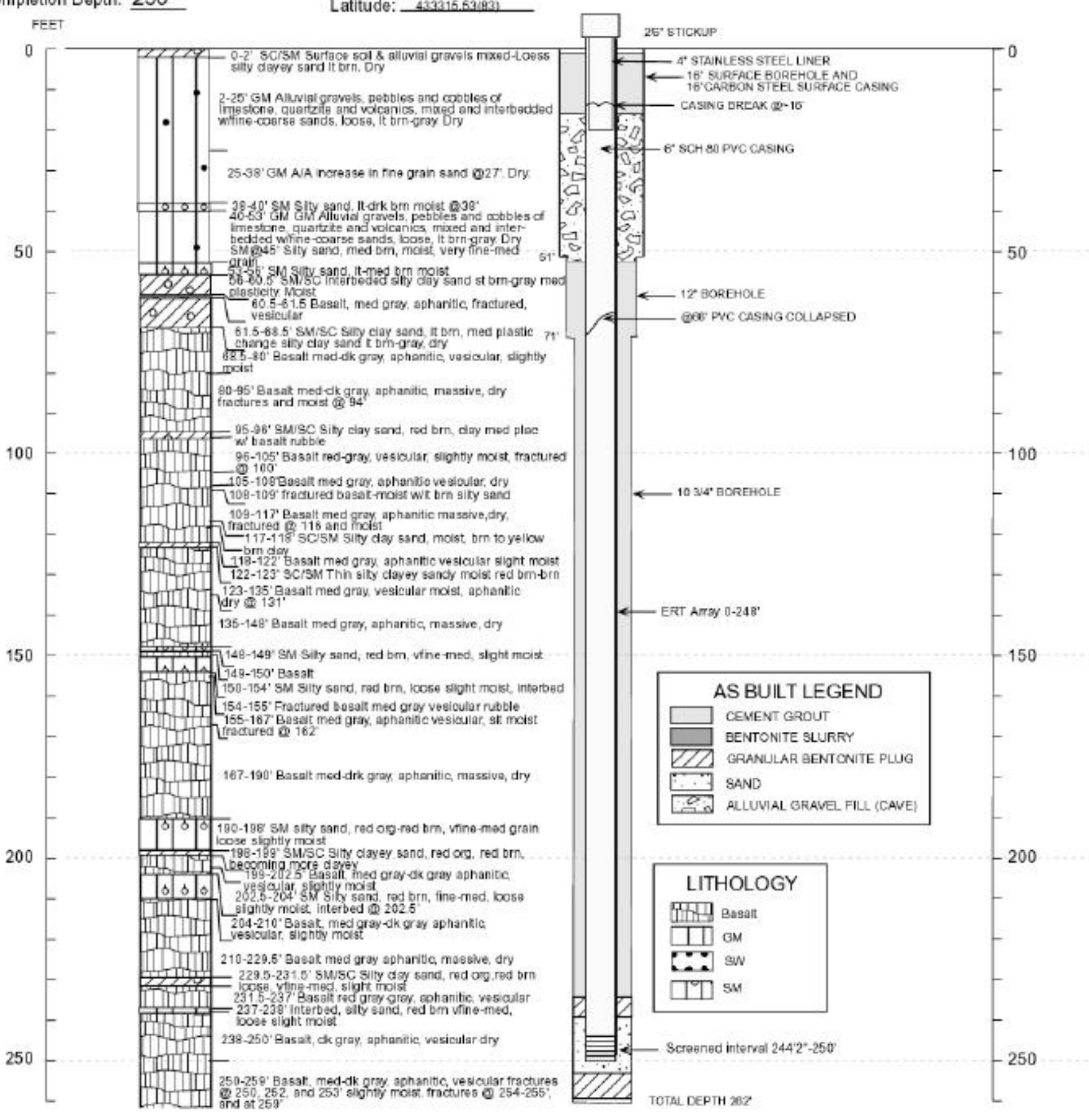

\begin{tabular}{|}
\hline \\
\hline
\end{tabular}

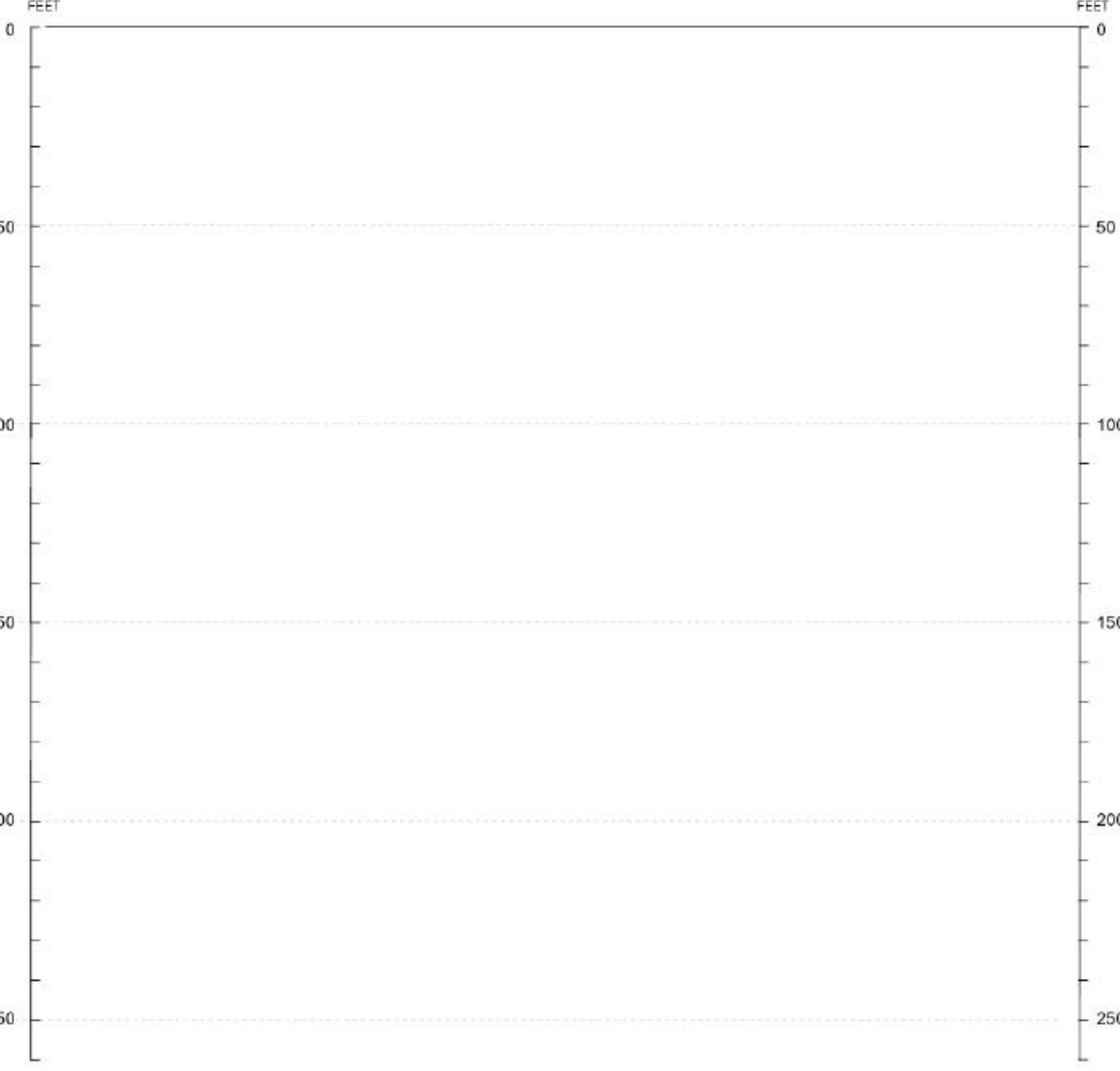


WELL NAME. ICPP-MON-V-202

INTEC (Perc Ponds)

First Interbed Monitorin

Well Status Abandoned

Year Drilled: 2000

Easting:-

Driller:DynateciRoy Brown Today Oate 1/10/0

Total Depth $136^{\prime \prime}$

Northing-

Gellogist. Gater Level: na

Longitude:

Drill Method: Reverse Air Rotaly

Drill Fluid: Air

Water Level Date:

Completion Deptr: ${ }^{131.25^{\circ}}$ Land Surface:

Water Level Access: na

tart Date $10 / 24 / 00$

10/25/00
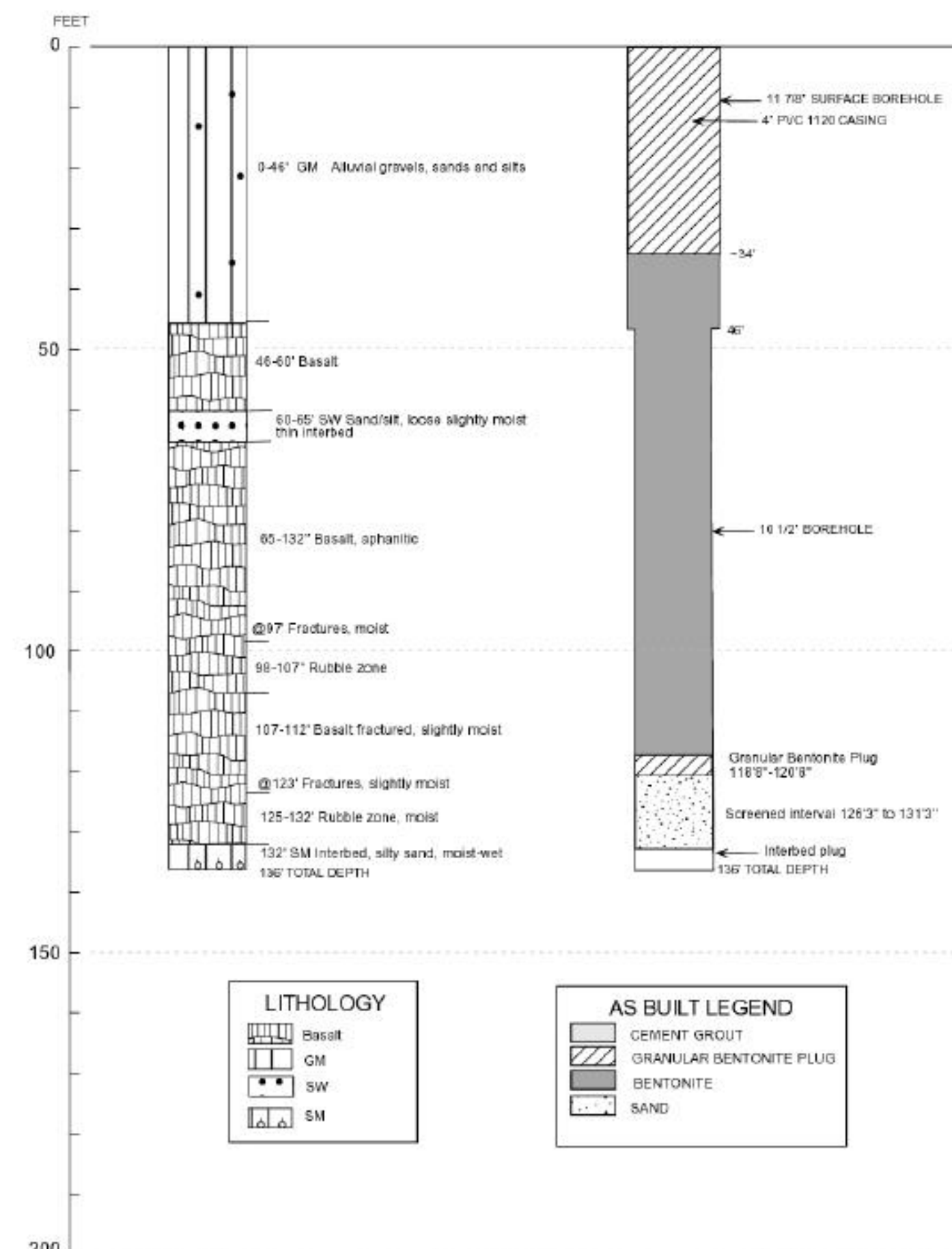

\begin{tabular}{|c|}
\hline \multirow{2}{*}{ 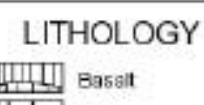 } \\
\hline \\
\hline$\bigcup_{\text {sw }}$ \\
\hline It \\
\hline
\end{tabular}

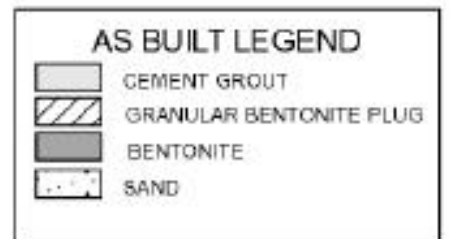

(IT)

…

IIIIII

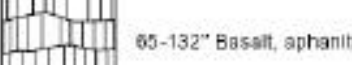

(III)

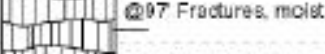

IIIIII

IIIIIII 107-112' Easaltractured, sliaraly mois

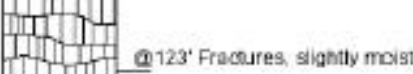

7IIIIII 125-122' Rubble zone, rols

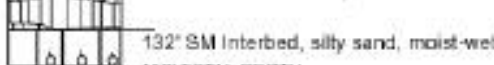

BENTON

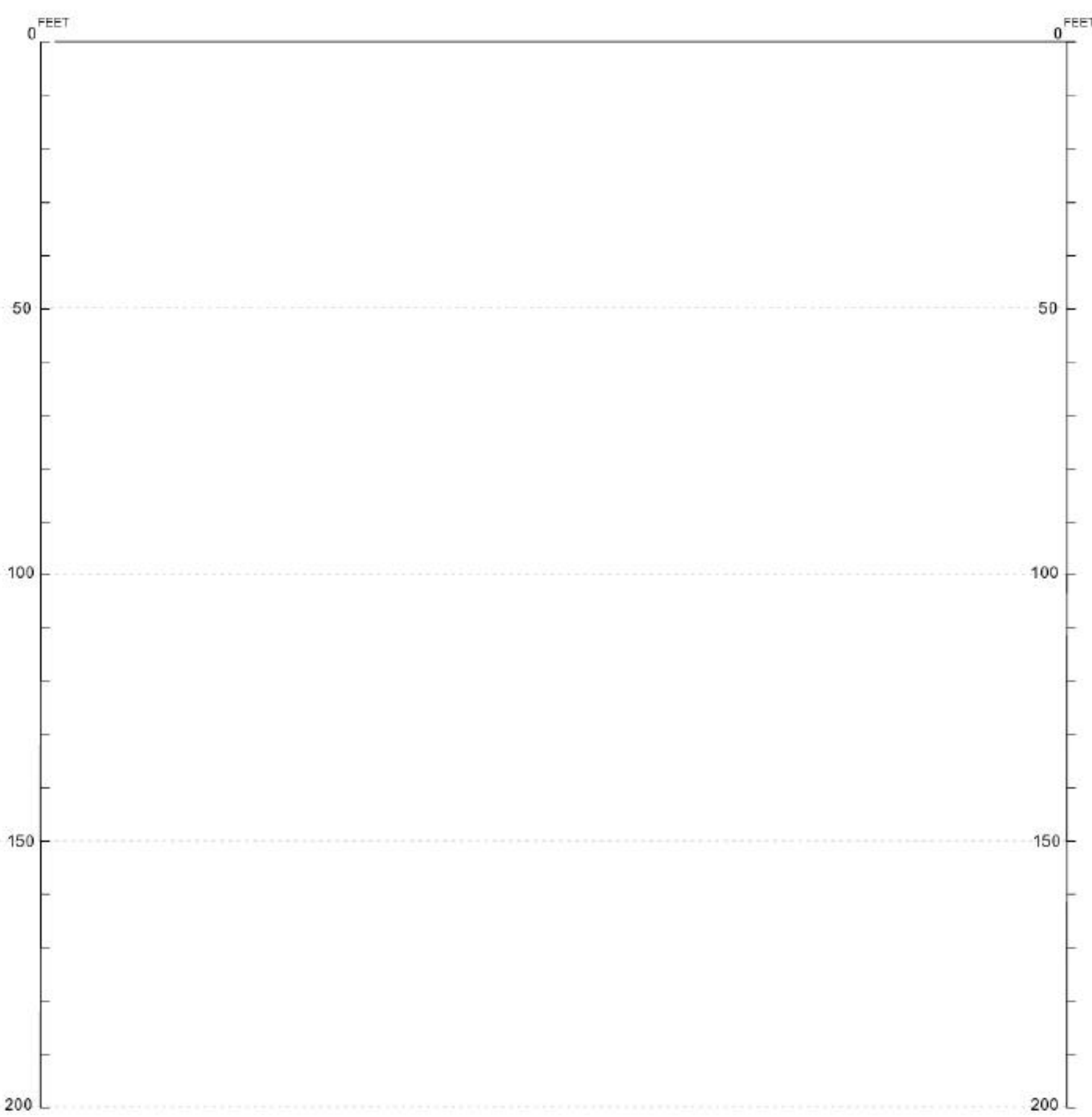


WELL NAME: ICPP-MON-V-202A
Fadilty: Perc Ponds

Well Type: First Inter

Brass Cap

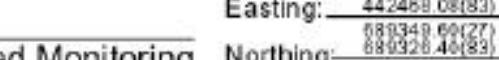

Well Status Active

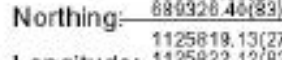

Driller. DynatedRoy Brown Todays Date: 1/10/01

Year Drilled: 2000

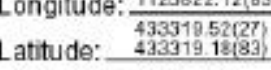

Geologist G. Obenhansley

Water Level: na

Total Dopth 123'9" Lattude: 42331918

Drill Fluid:Air

End Date 10/30/00 Water Level Access: na

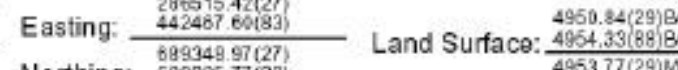

Completion Depth: $122^{\prime}$
EEET

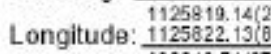

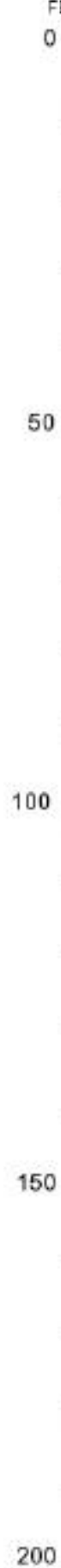

200

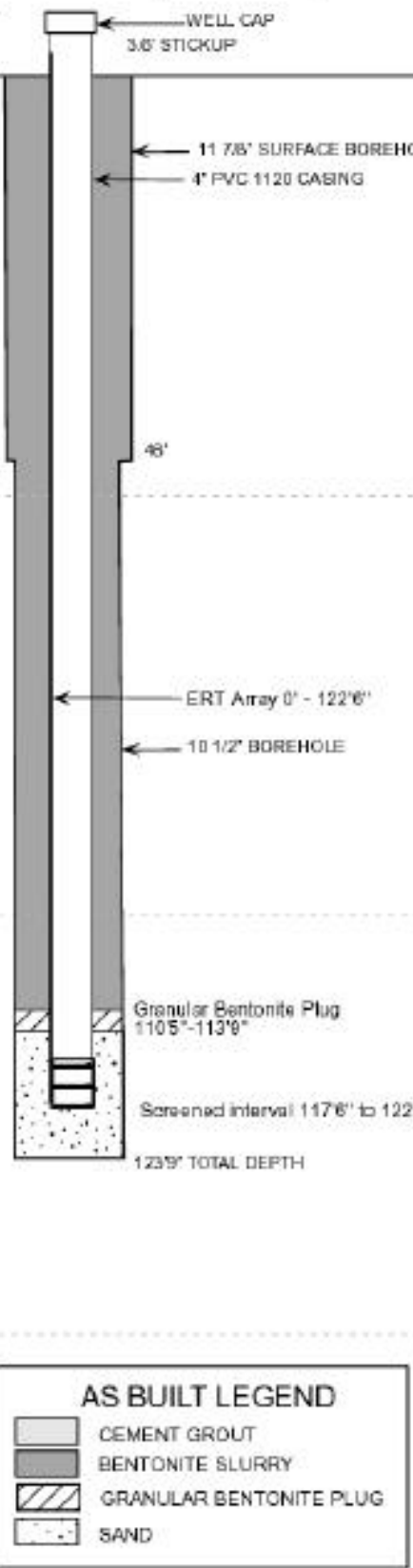

200

$$
\begin{aligned}
& \text { LITHOLOGY }
\end{aligned}
$$

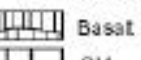

$$
\begin{aligned}
& \text { प्:D }
\end{aligned}
$$

Natural Gamma

$\underset{\substack{\text { Neutron } \\ \text { AFIN }}}{\cos }$

Gamma Gamma

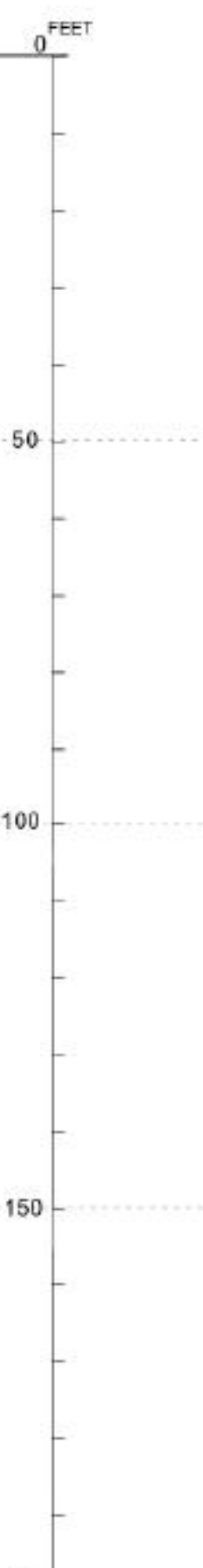

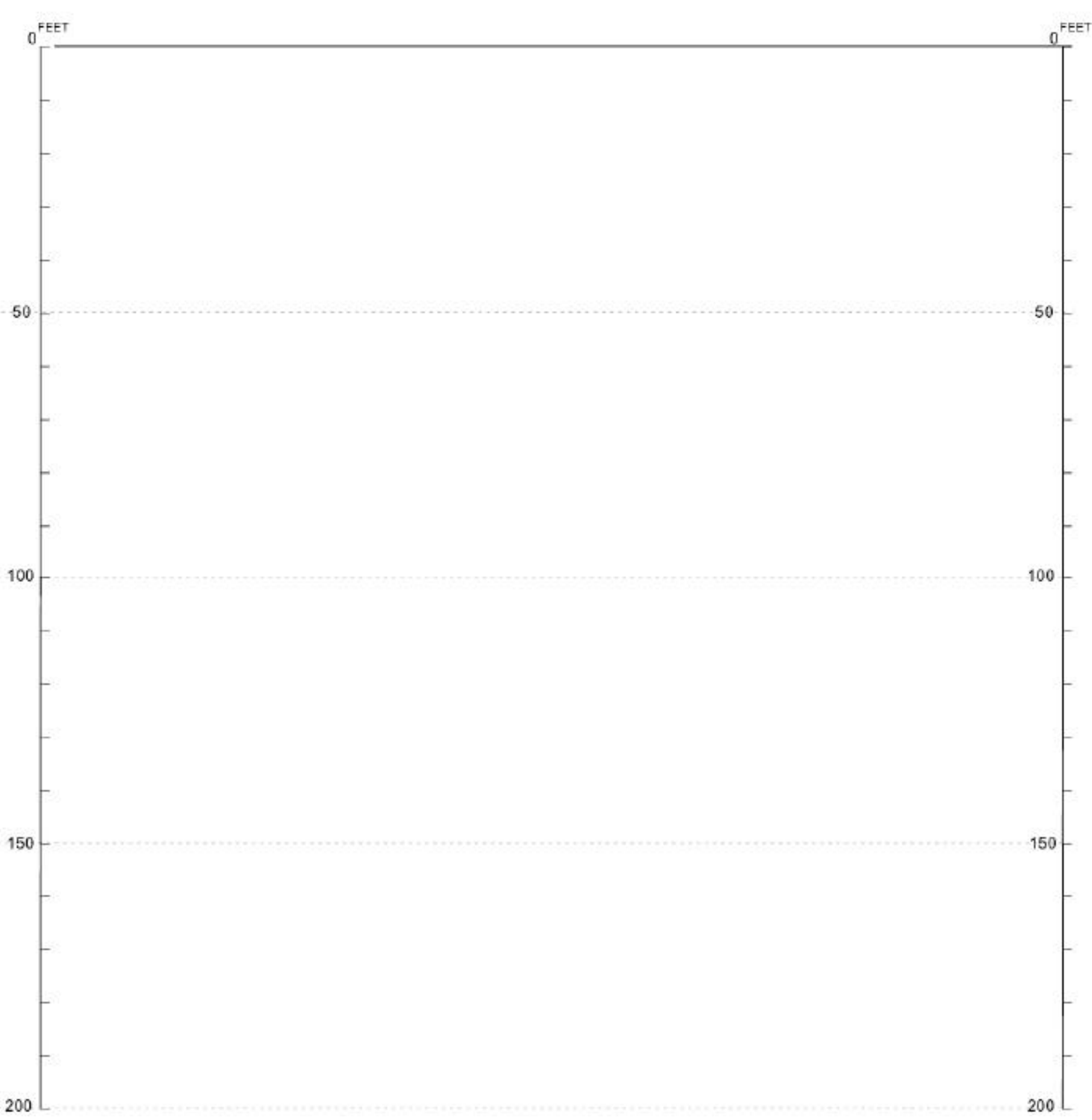


WELL NAME: ICPP-MON-S-203

Facility, Perc Ponds

Well Type, Active

Weall Status $\frac{\text { Active }}{2000}$

Total Depth 54

Start Date $10 / 31 / 00$

End Date $10 / 31 / 00$

Completion Depth:

0

100

LITHOLOGY

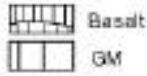

$$
\text { FE }
$$

Brass Cap

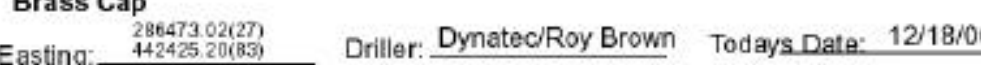

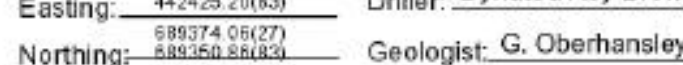

Water Level. na

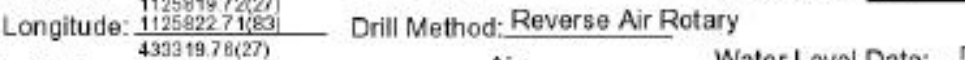

Latitude: 433319.4262837$)$ Drill Fluid: Air

Water Level Date: na

Measuring Point

Water Level Accoss: na

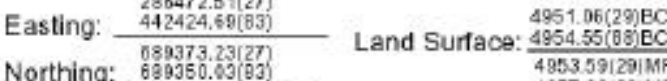

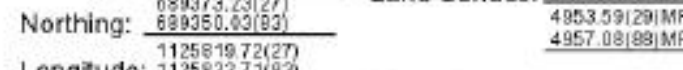

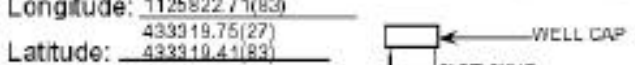

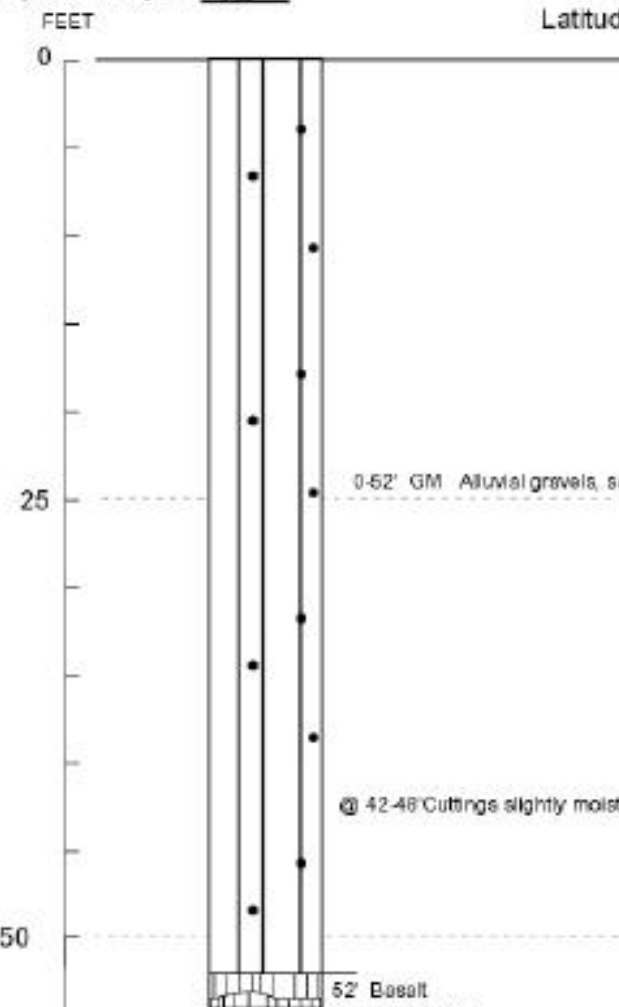

IIIn⿴囗十) 52 Eoealt

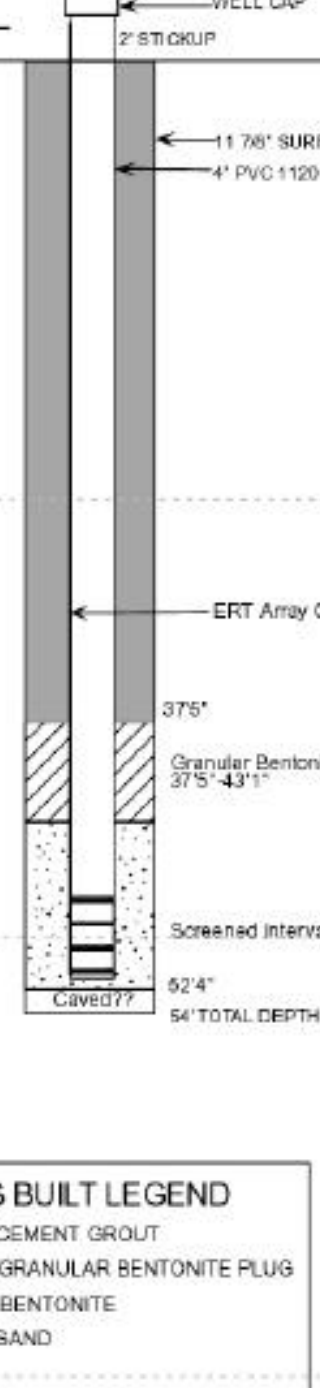

$\underset{A P l . C Q}{\text { Natural Gamma }}$

Gamma Gamma

$\underset{\text { Caliper }}{\text { INCH }}$

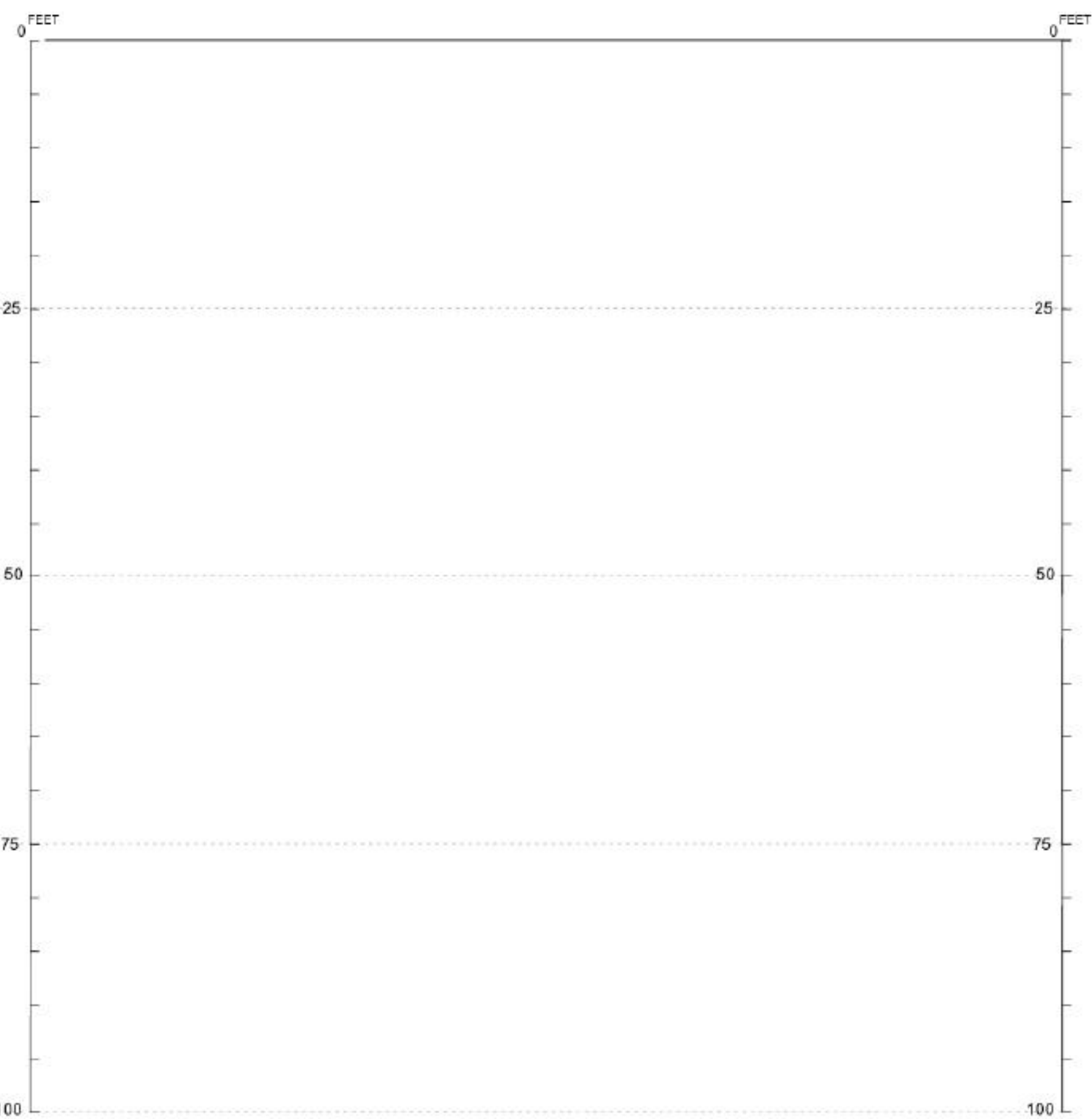


WELL NAME. ICPP-SCI-V-204 Facility: INTEC (Perc Ponds) Well Type: Instrumented

Well Statusctive

Year Drilled2001

Total Depth 1754"

Start Date $4 / 02 / 00$

End Date 4/11/00

Completion Depth: $174^{\prime 2} 3^{\prime \prime}$

\section{Brass Cap}

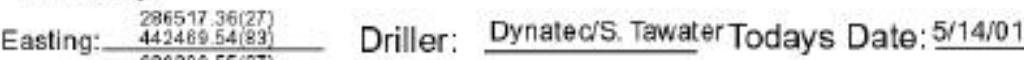

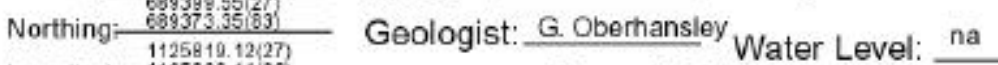

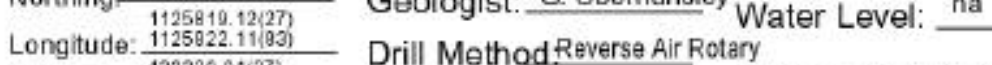

433320.01127 ) Drill MethodReverse Air Rol

Water Level Date: na

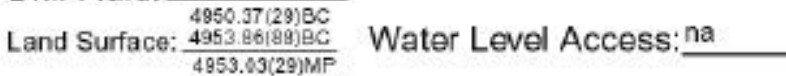

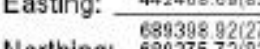

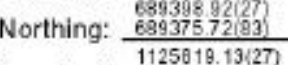

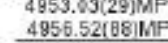

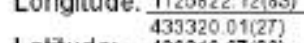

FEET

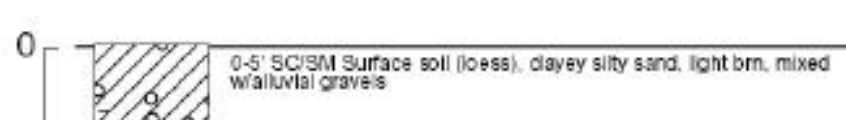

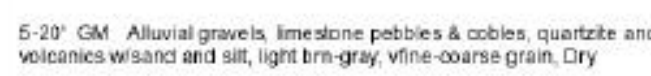

40. AA sighthy moist

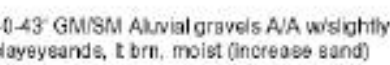

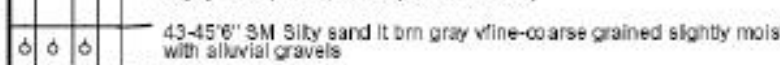

$50-$

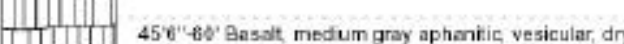

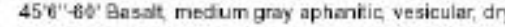

(1)

\begin{tabular}{lll|l}
0 & 0 & 0 & \\
0 & 0 & 0 & 0
\end{tabular}

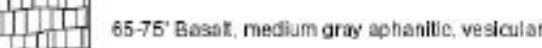

페민.

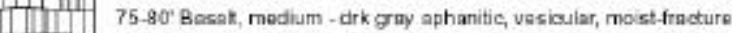

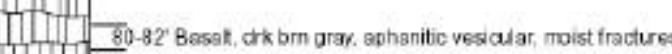

1)

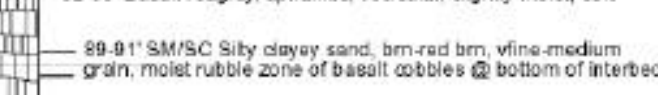

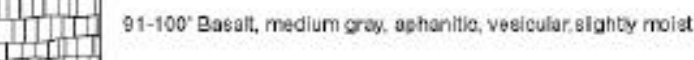

100

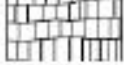

cortinusd on page 2

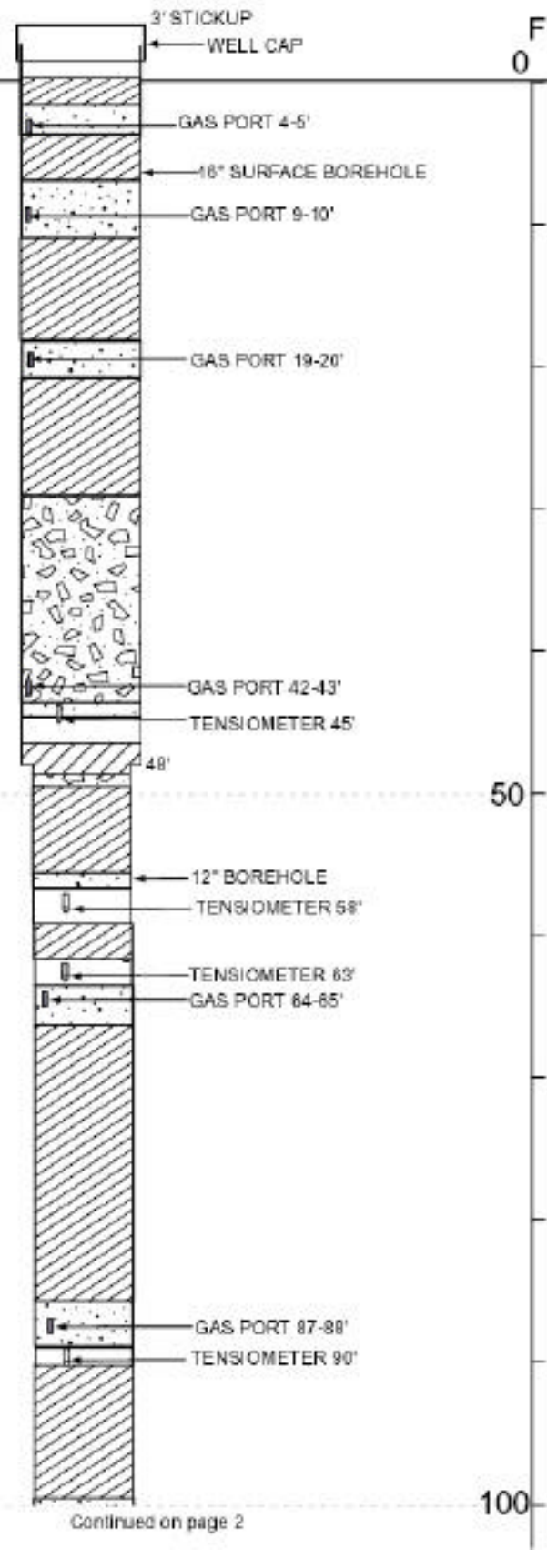

$\underset{\text { Nal-GR }}{\text { Natural Gamma }}$

$\underset{\text { Neutron }}{\text { APIN }}$

Gamma Gamma 
WELL NAME.ICPP-SCI-V-205 Facility: INTEC (Perc Ponds) Well Type: Instrumented

Well Statusctive

Year Drilled2001

Total Depth 162'6"

Start Date $2 / 28 / 01$

End Date $3 / 8 / 01$

Completion Depth: 162'6"

FEET

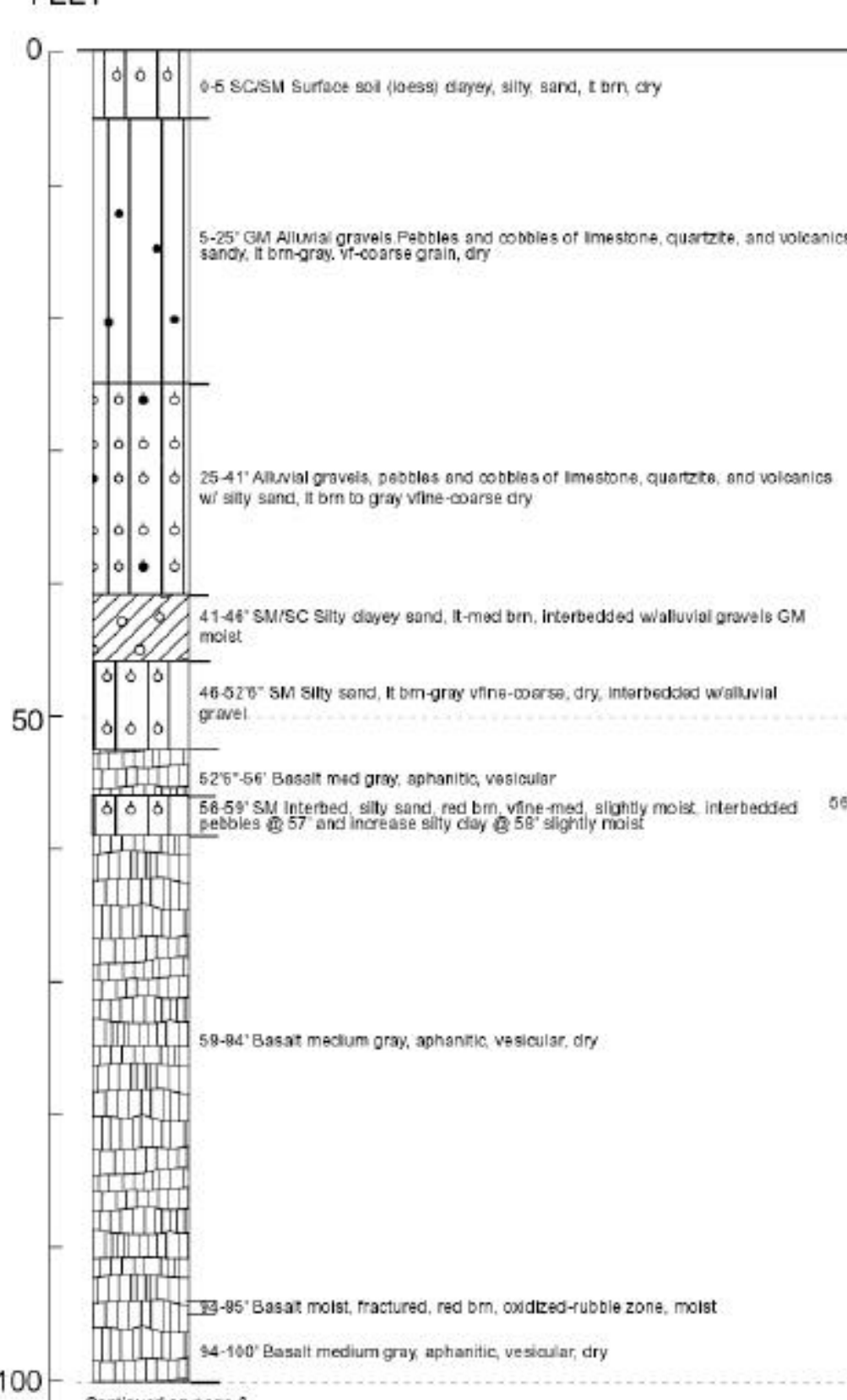

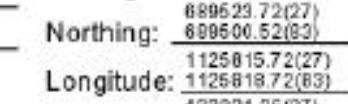

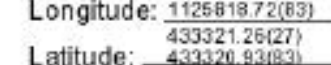

Brass Cap 2865692527$)$ Driller: DynatedS. Tawater Todays Date: 6/25/01

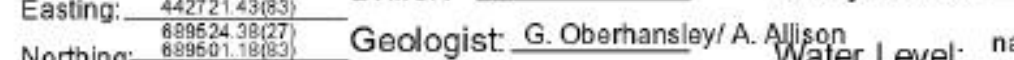

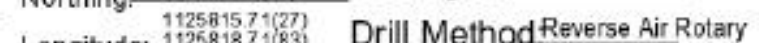

439321.27.277

Measuring Point

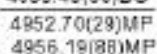

$\underset{\text { Natural Gamma }}{\text { API-GR }}$ $\underset{\text { APF-N }}{\text { Neutron }}$

$\underset{\text { GPS }}{\text { Gamma Gamma }}$

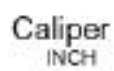

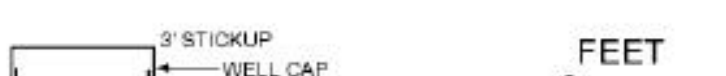

FEET

. 1000

○ $1000 \quad 2000$

- $1000002300000 \quad 30000$

FEET

GaS PORT 19-20'

ili 19

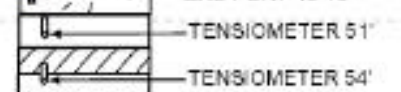

Tensometer 54'

TENIOMETER 5T

CAs PORT 6253

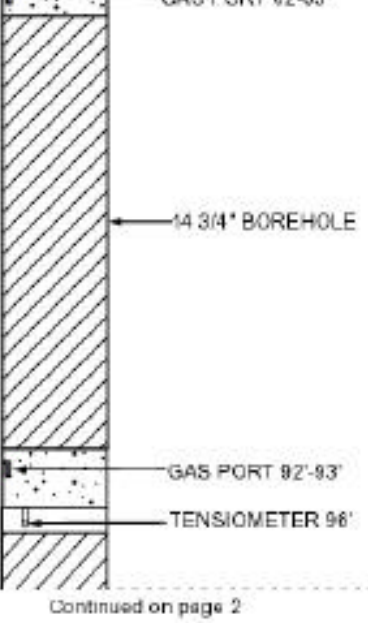

50

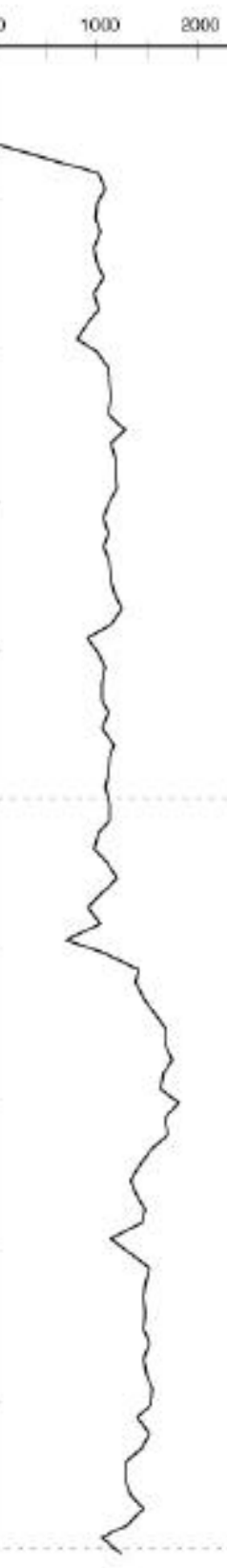

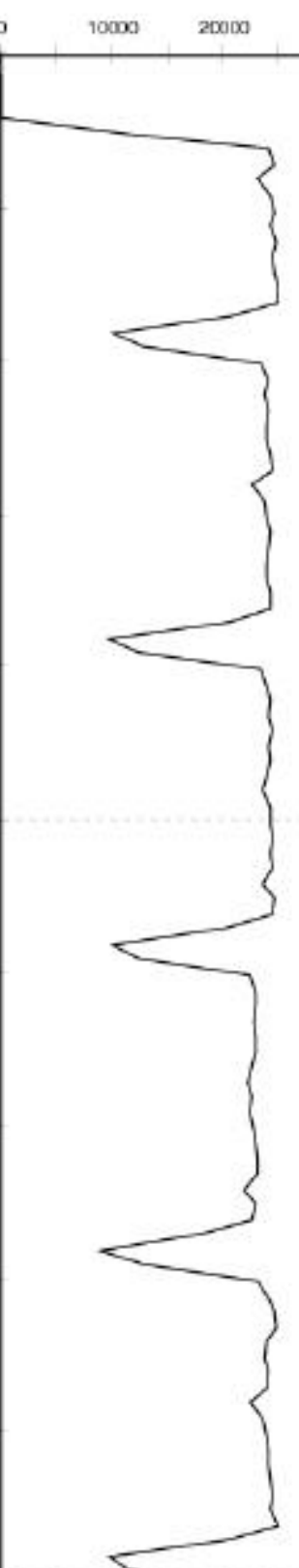

50 


\section{WELL NAME.ICPP-SCl-V-207 \\ Facility: INTEC (Perc Ponds)}

Well Type: Instrumented

Well Statusctive

Year Drilled2001

Total Depth 150'

Start Date 4/17/101

End Date 4/24/01

Completion Depth: $150^{\circ}$

$$
\text { FEET }
$$

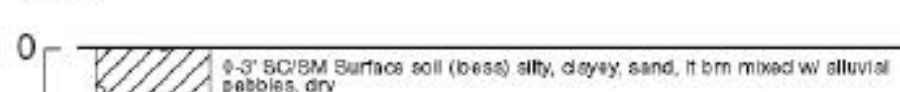

$50-$

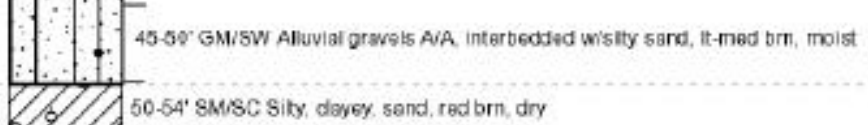

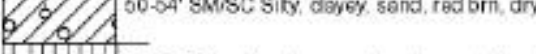

IIIIIIII ${ }^{54-58}$ ' Basalt med grew, apharitic vasioulardidy

101.10

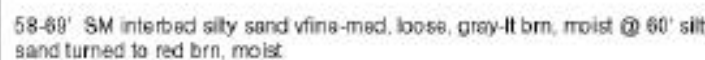

.10 .0

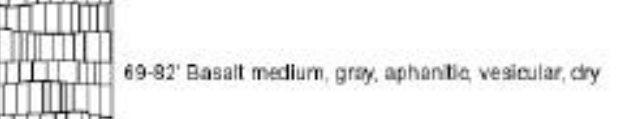

IIIIII

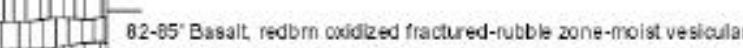

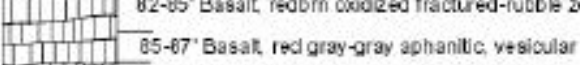

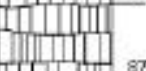

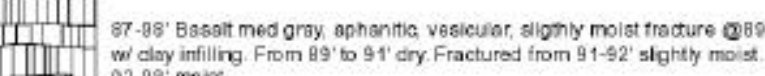

IIt

$100-$

corrinues on pasge 2
Driller: DynatedS. Tawater Todays Date: 6 6/26/0

Geologist: G. Oberhansley/ A, Allison

Drill MethodReverse Air Rotary

Drill Fluid: Alr

Water Level Date: na

Water Level Access: na

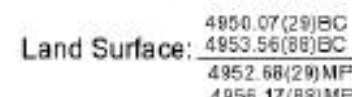

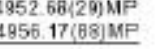

$\underset{c \text { Gas }}{\text { Gamma }}$

Caliper

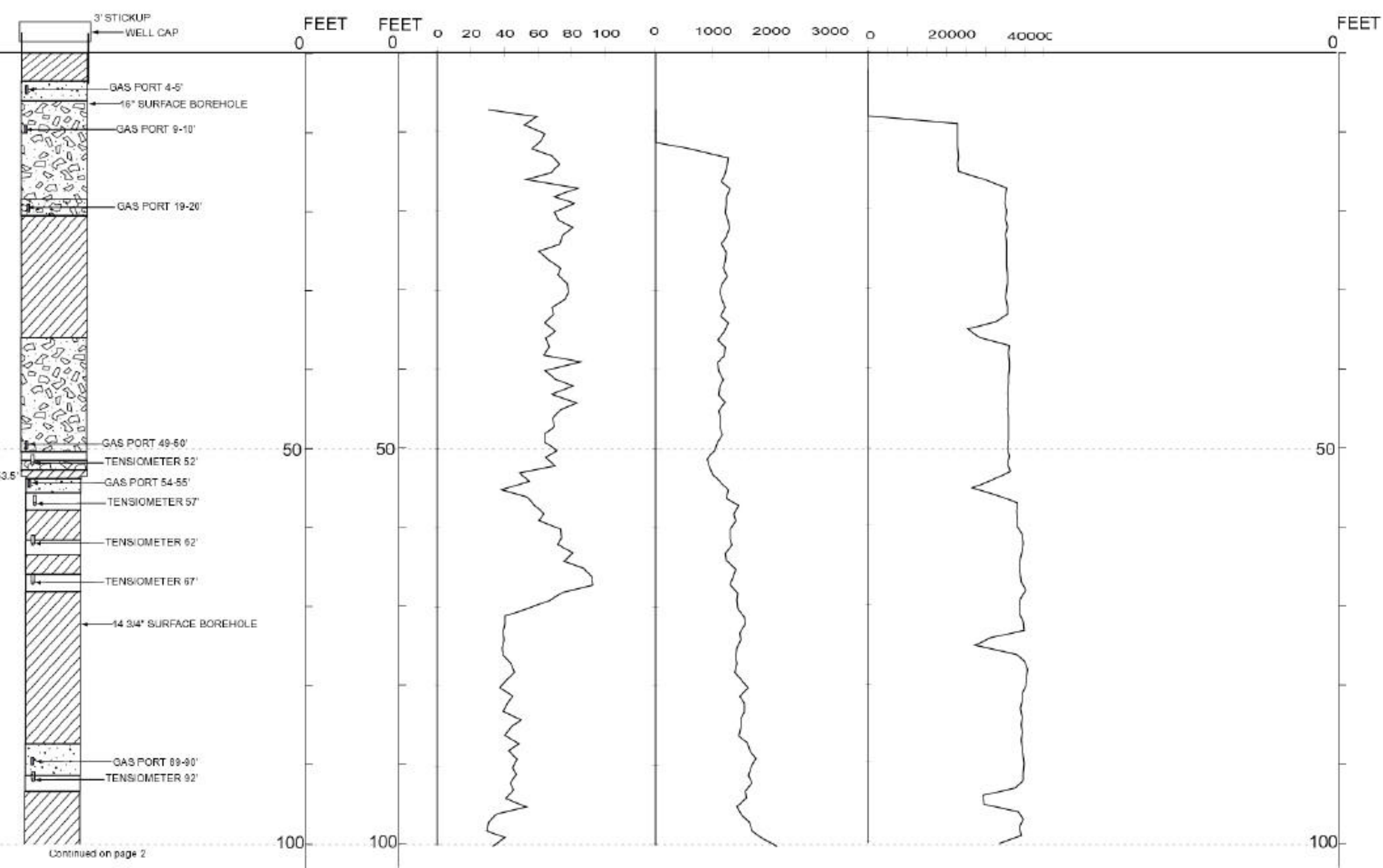


Well Type: Instrumented

Brass Cap

Well Status Active

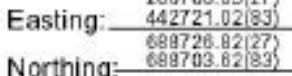

Total Depth 1626"

Driller: Dynatec/S. Tawater Todays Date: $6 / 25 / 01$

Start Date 2228/0 Geologist. G. Obenter Level: na

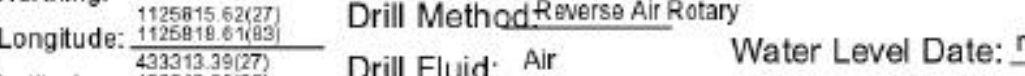

End Date 3/8/01

Me asuring Point Water Level Access:na

${ }_{4550.07(29) 190}$ Water Level Access:

Natural Gamma

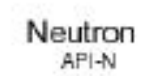

$\underset{\mathrm{CPS}}{\text { Gamma Gamma }}$

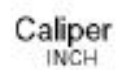

FEET

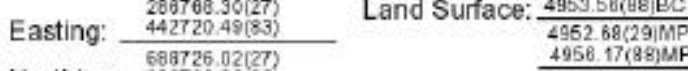

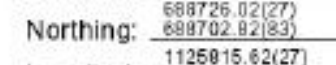

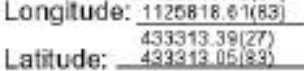

FEET

FEET, $20 \quad 40 \quad 60 \quad 80 \quad 100 \quad 0 \quad 1000 \quad 20003000$

$20000 \quad 40000$

100 Continued from arevibus pase

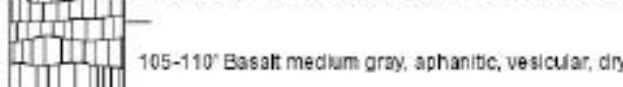

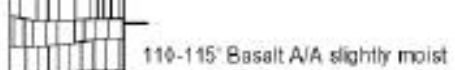

IIIIII

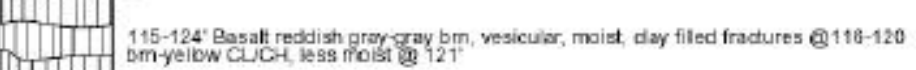

niti-

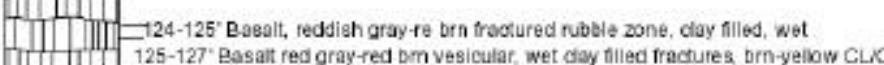

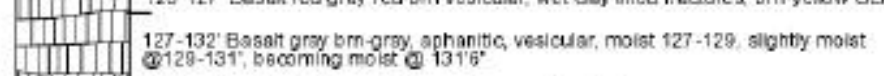

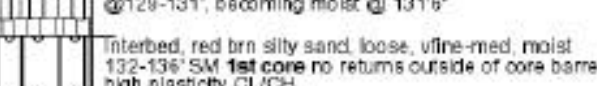

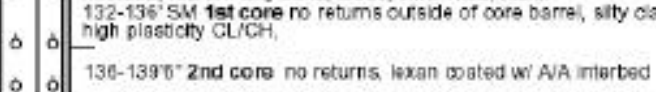

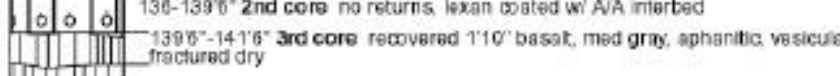

Tín

150

conthued from previous page

$100 \quad \begin{array}{r}0 \\ 1\end{array}$
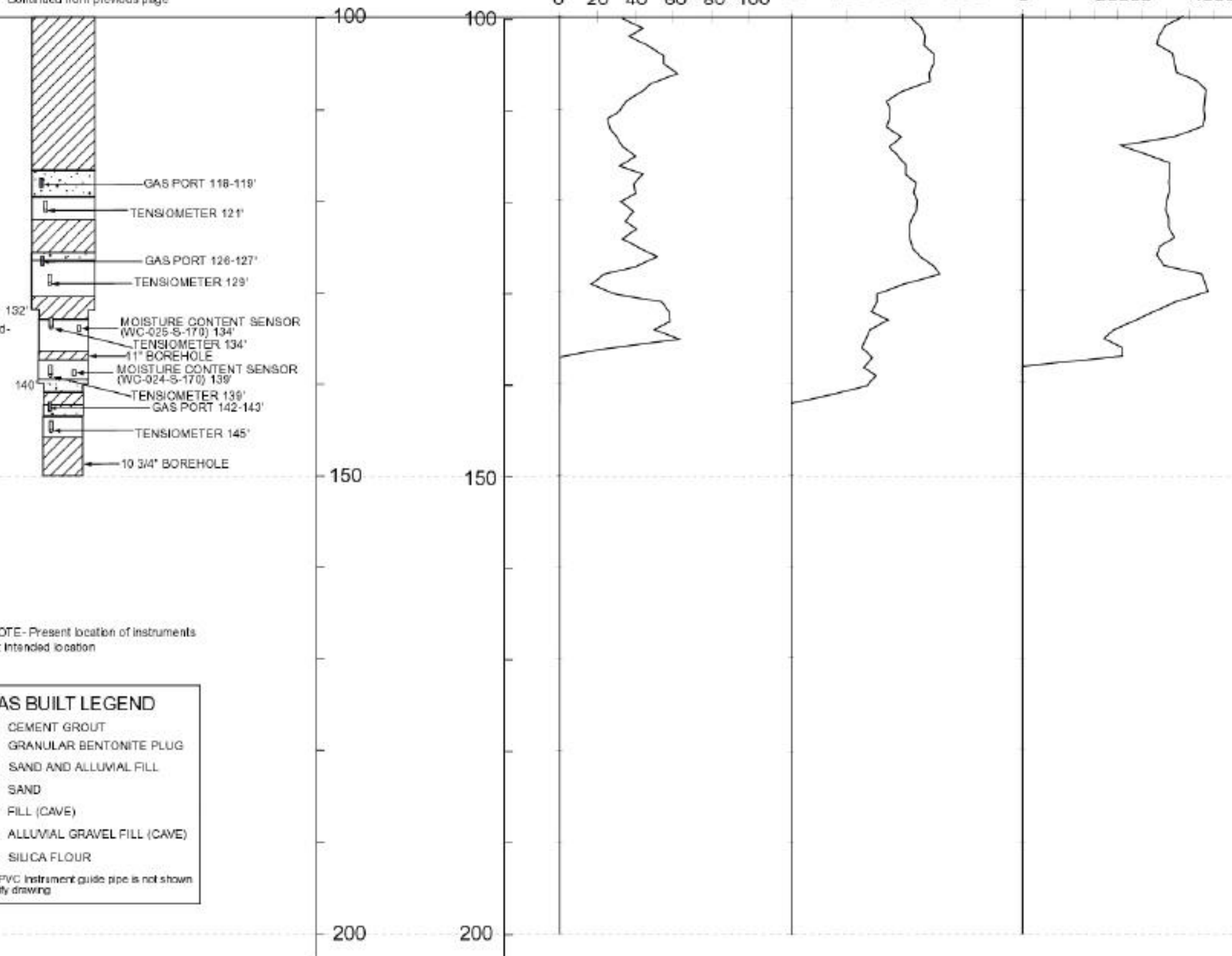

Nore-Pregent bcaton of instuments

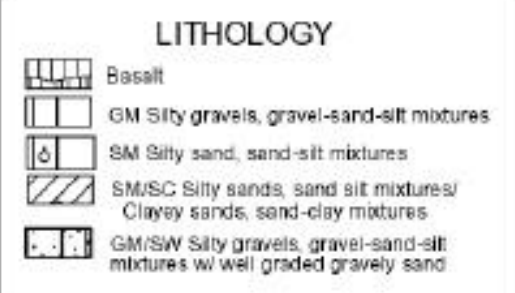

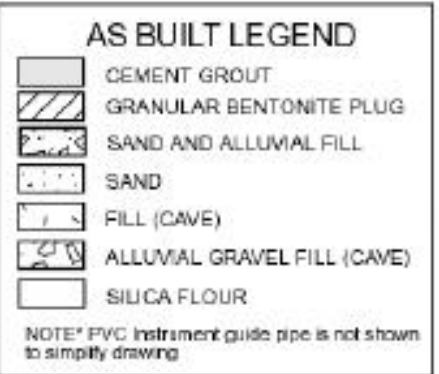

200

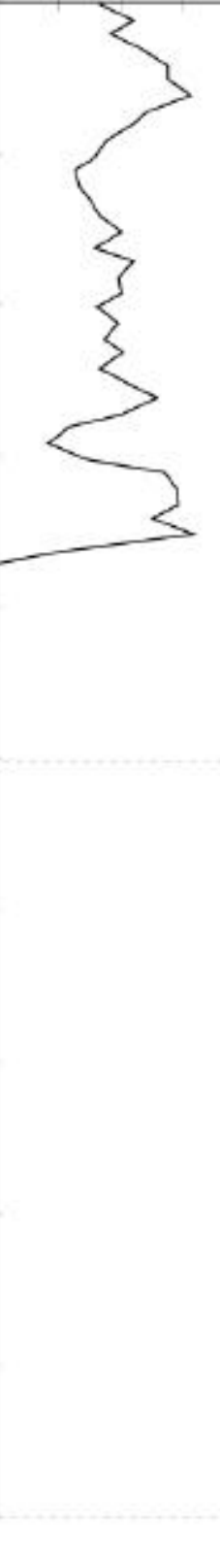


Brass Cap

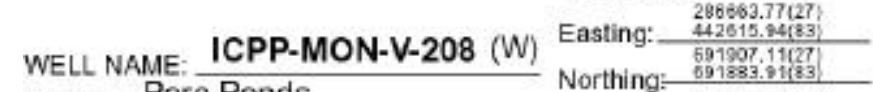
Facility. Perc Ponds
Well Type: Instrumen

Well Type.

Well Status Active/Developed Measuring Point

Year Drilled: 200

Total Depth $121^{\prime}$ Me asuring Point

Driller: Dynatecis. Tawater TodaysDate: 216/01

Gologist:G. Obenhansioy $W$

Water Level Date: 1/901

Drill Fluid. Air

Wartovidan.

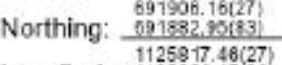

$\begin{array}{ll}\text { Start Date } & \frac{1 / 5 / 01}{1 / 51} \\ \text { End Date } & 1 / 8 / 01\end{array}$

Completion Depth: $113^{\prime} 10^{\prime \prime}$
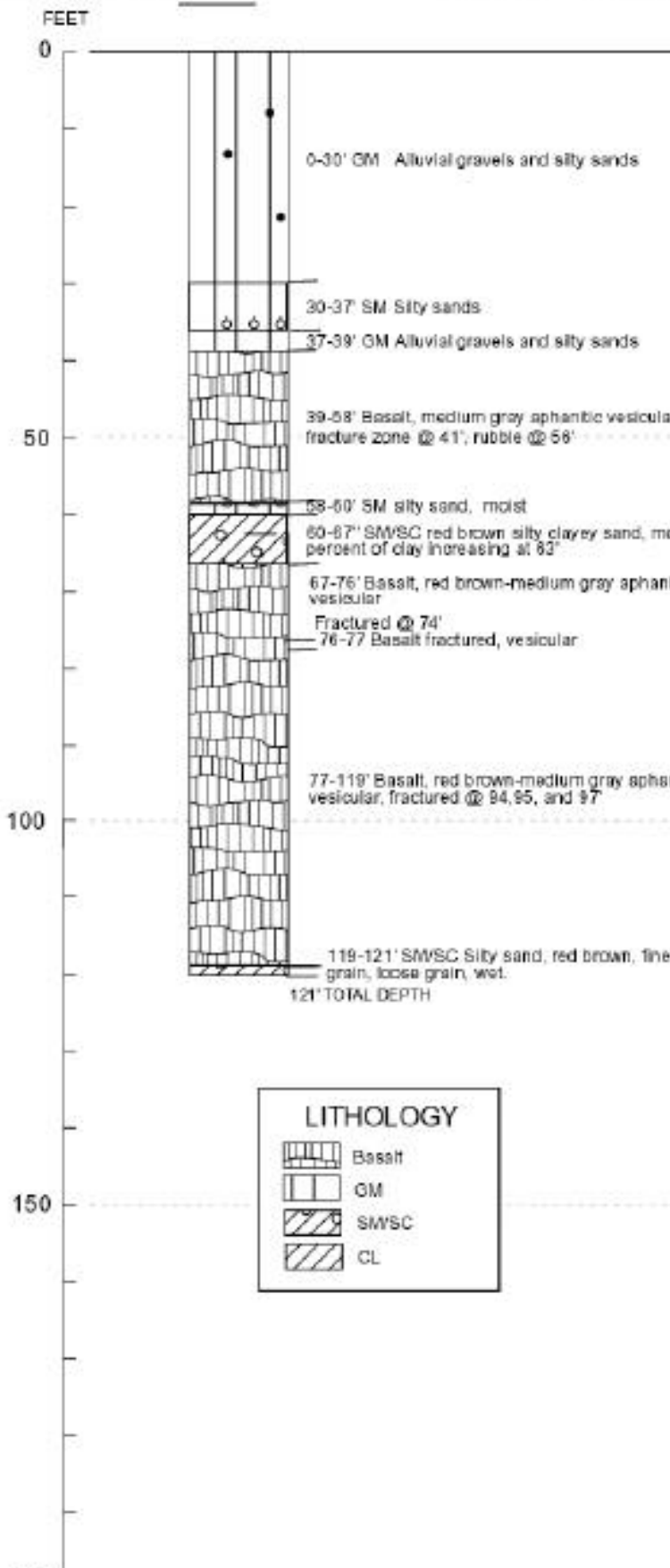

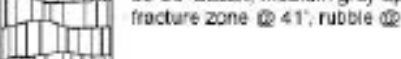

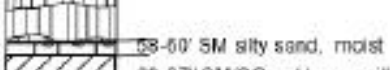

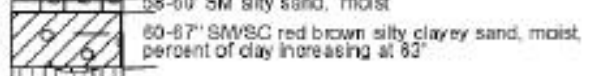

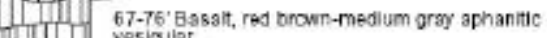

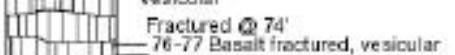

110

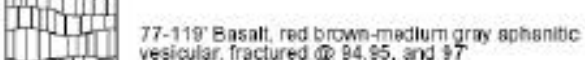

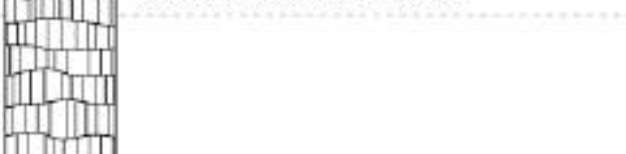

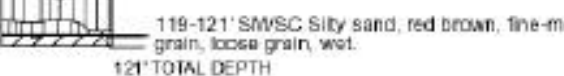

$$
\begin{aligned}
& \text { LITHOLOGY }
\end{aligned}
$$

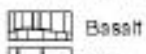

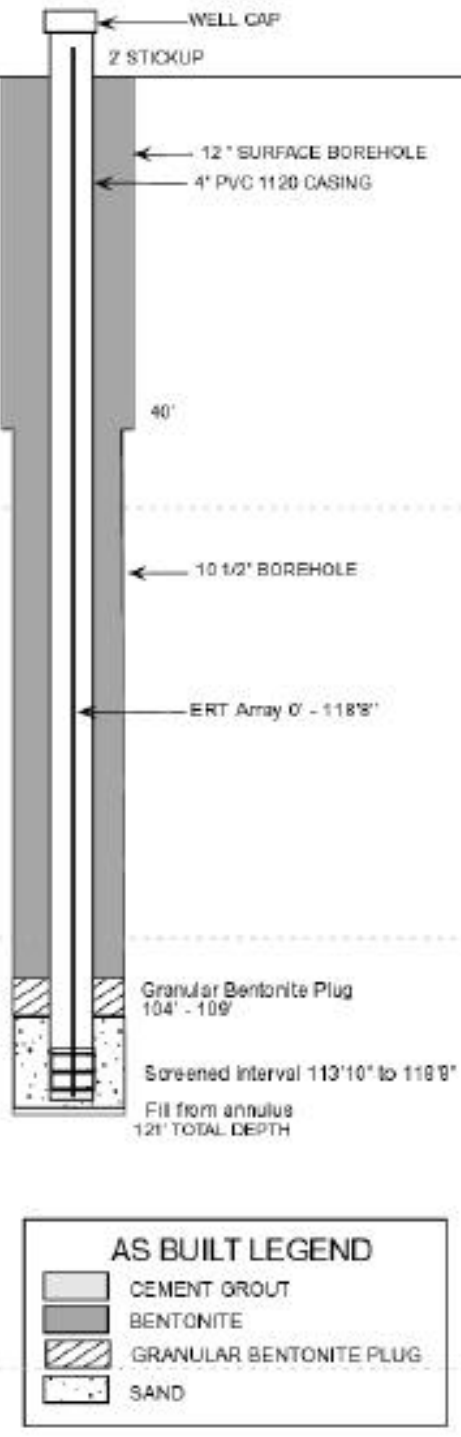

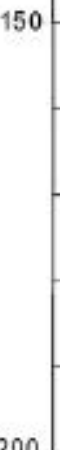

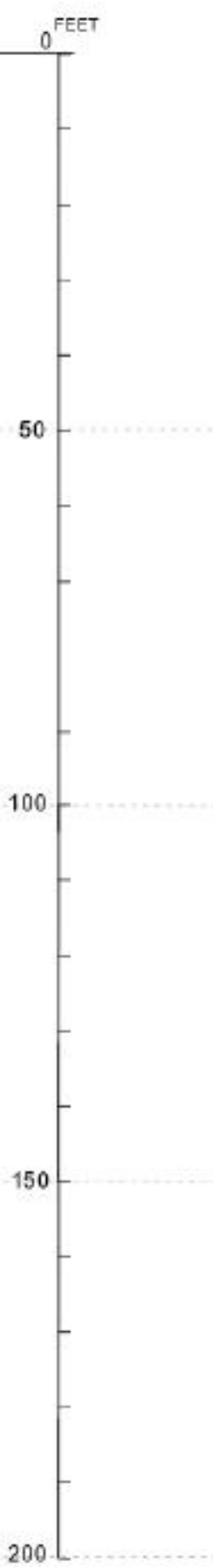

$\underset{A}{\text { Natural Gamma }}$

$\underset{\text { Neutron }}{A P I N \text { N }}$

Gamma Gamma

Caliper

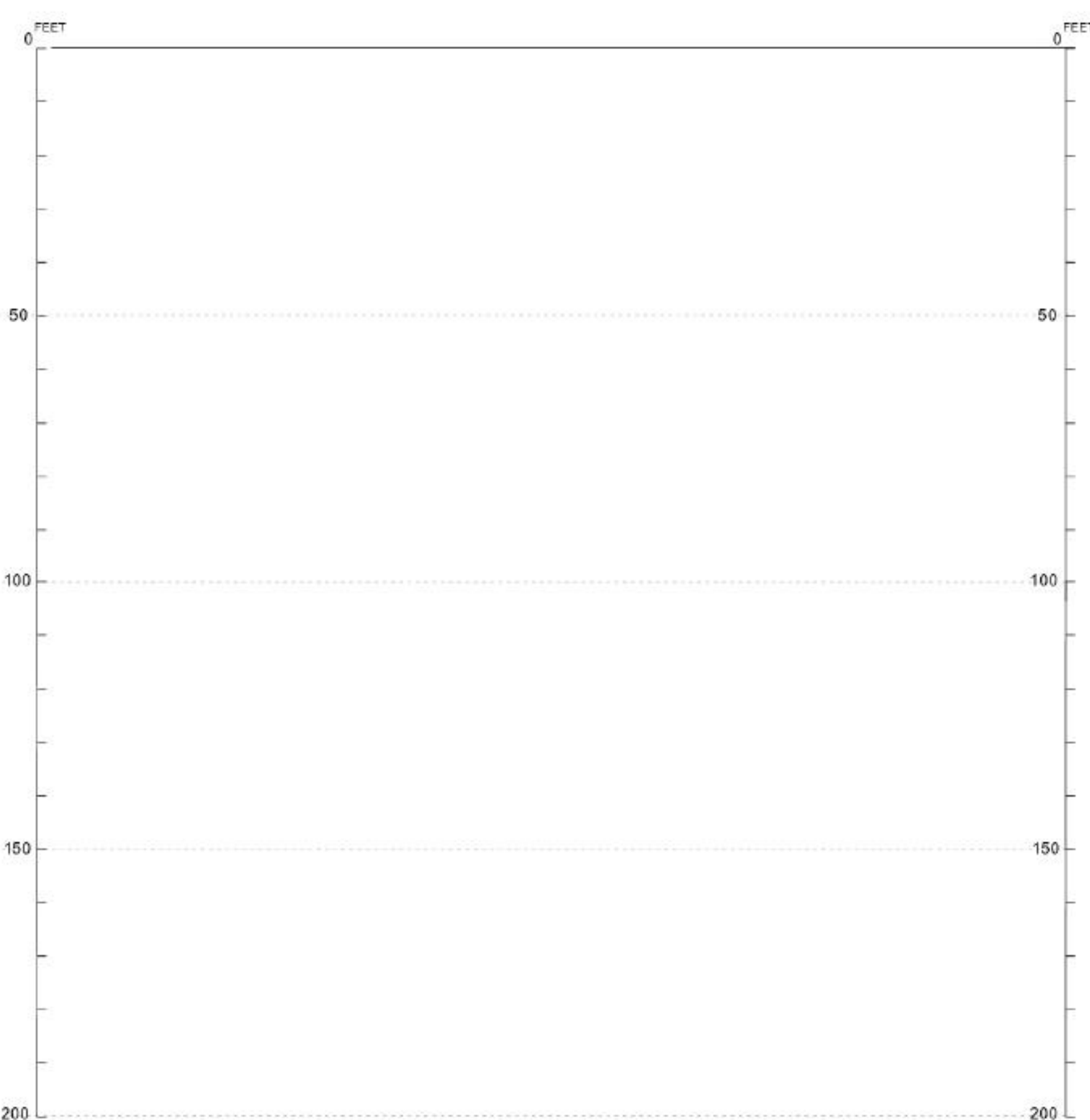


WELL NAMEE: ICPP-SCI-V-209

Brass Cap

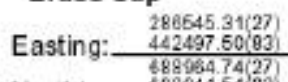

Instrumented Monitoring Northing:-

Well Status Active

Year Drilled: 2001

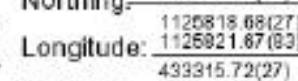

Total Depth 262' Latitude: 493935.598 Cid

Start Date $5 / 16 / 01$

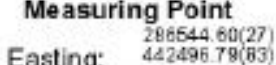

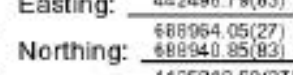

End Date $\quad 5 / 23 / 01$

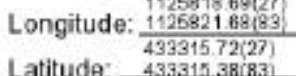

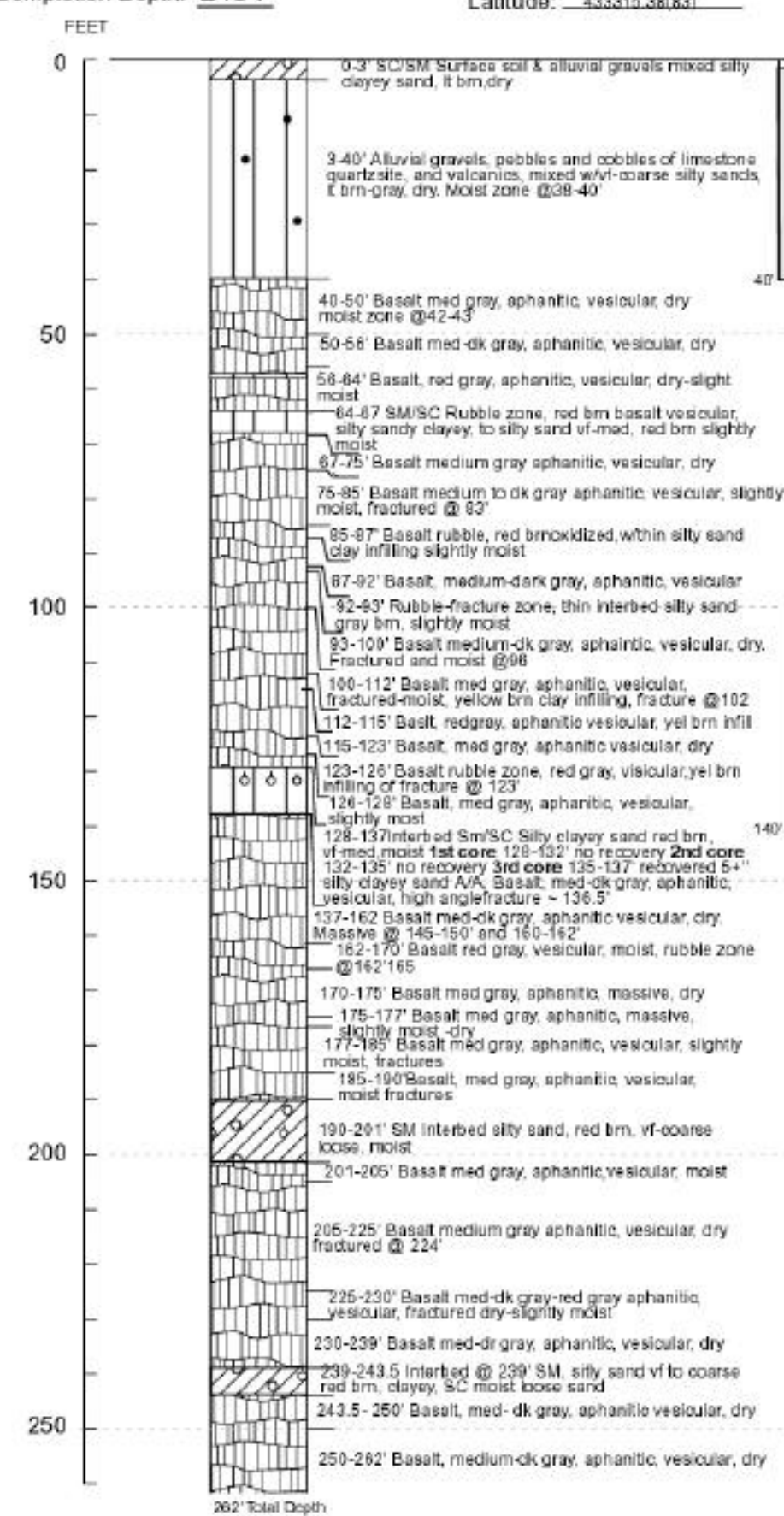

Dynatec/S. Tawaterl'
Driller:G. JensenjGrank Hight Todays Date: 8/9/01

Geologist:G. Obenhansley Wartor . na

Dill Method:Reverse Air Rotary Water Level Date: na Dill Fluid: Air $4950.711299 \mathrm{gC}$ Water Level Accoss: na

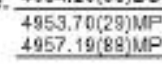

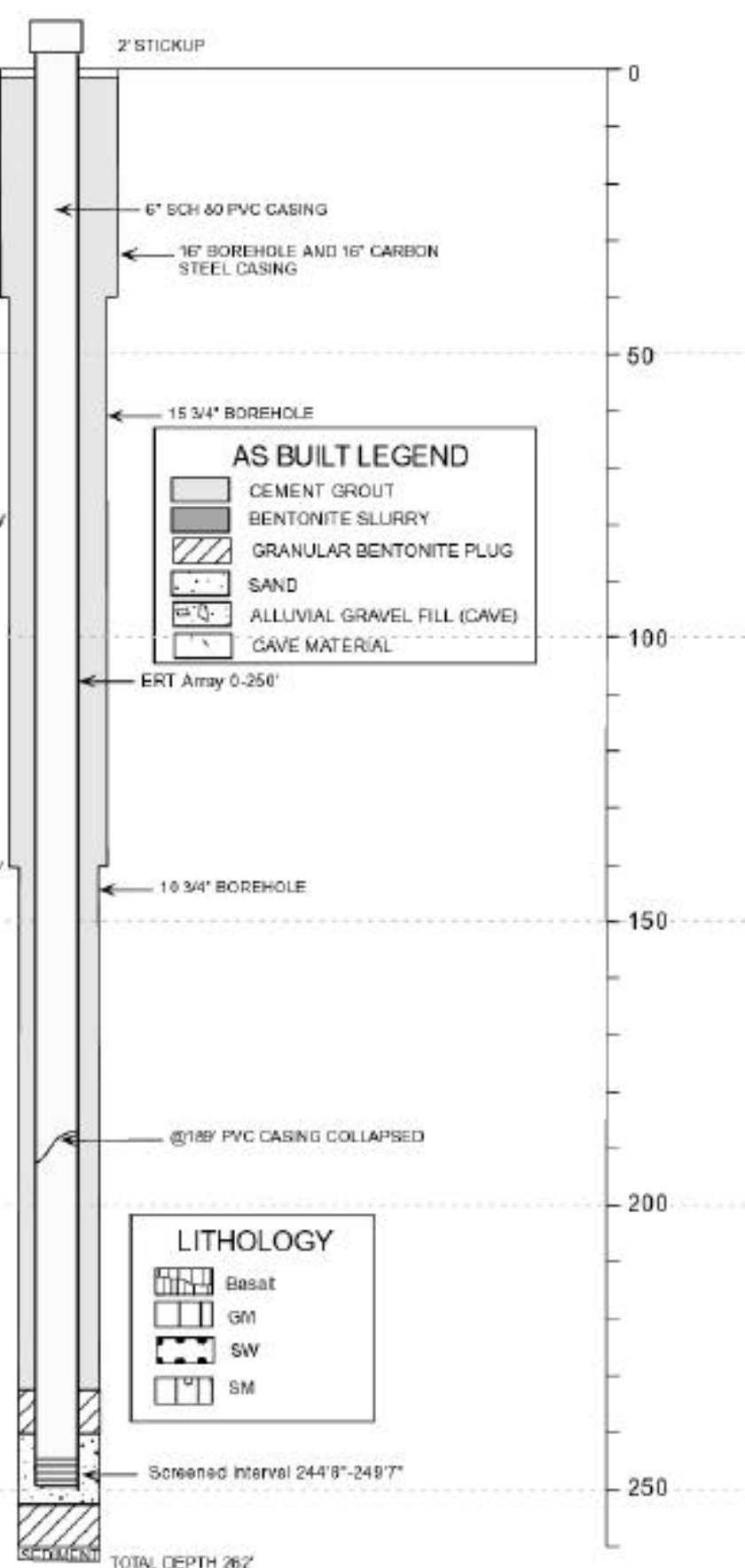

Natural Gamma Neutron Gamma Gamma

$\underset{\substack{\text { Caliper } \\ \text { INOH }}}{\text { SO }}$

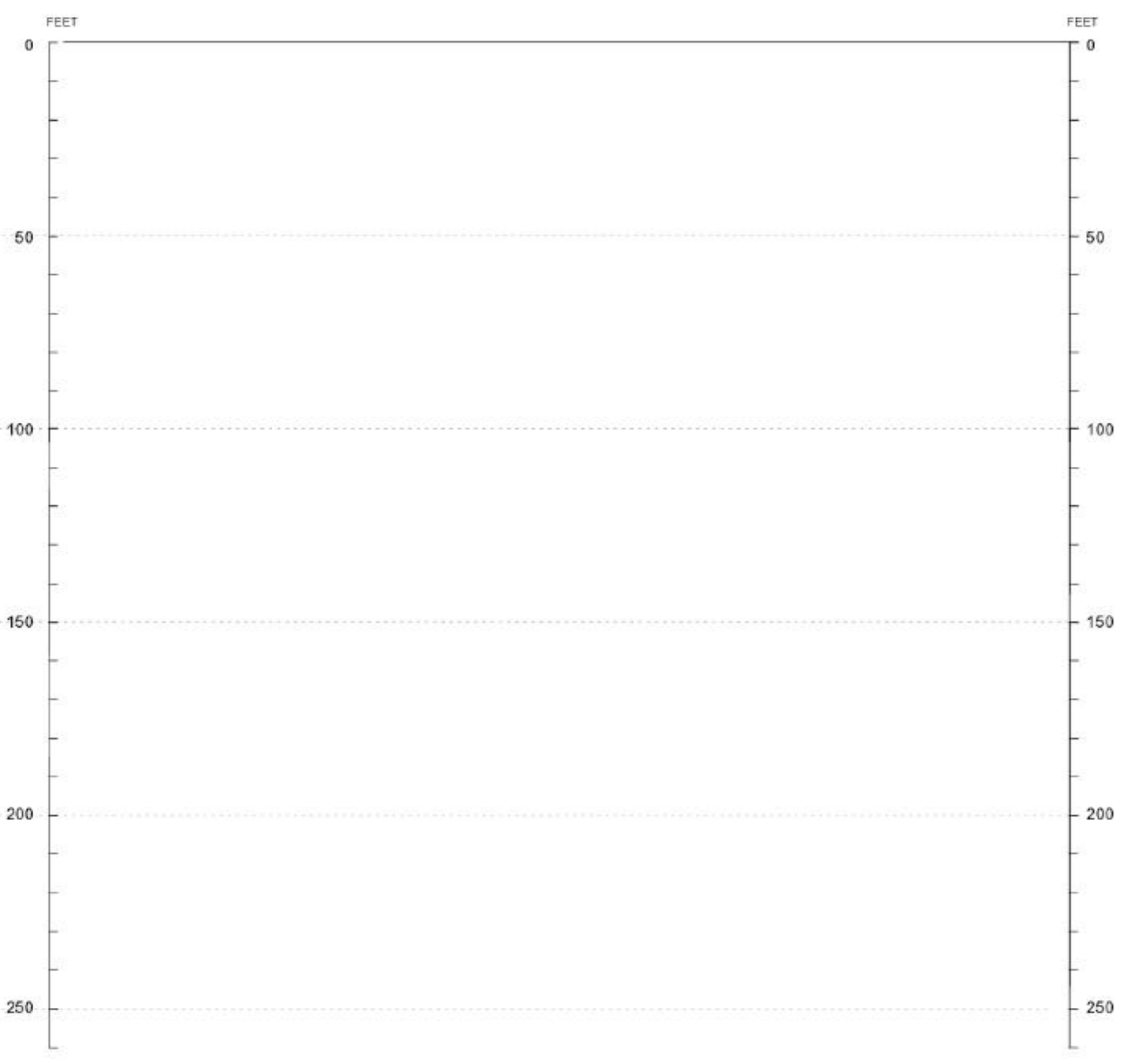




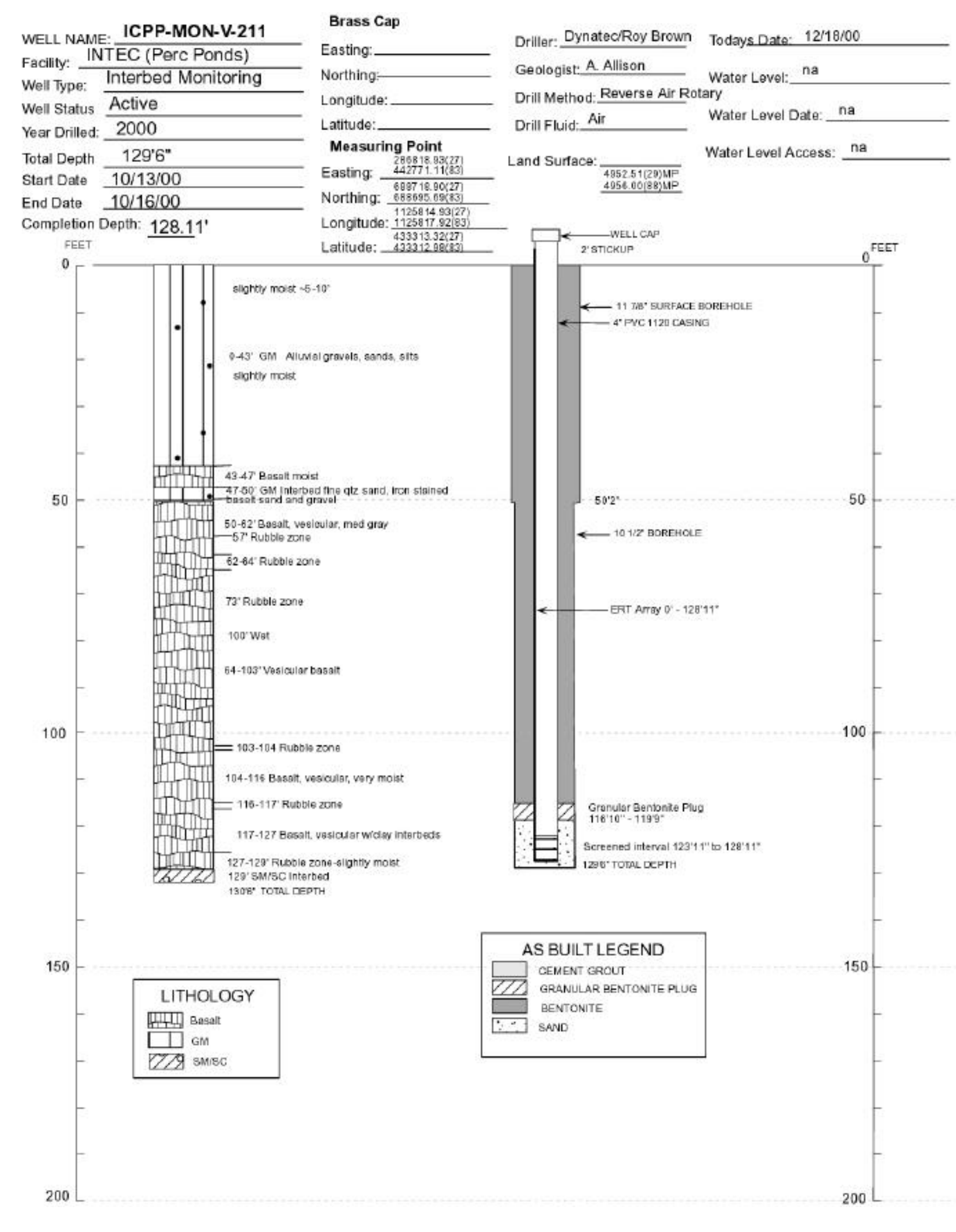

$\underset{\text { NP1-GR }}{\text { Natural Gamma }}$

$\underset{\substack{\text { Neutron } \\ \text { API-N }}}{\cos }$

Gamma Gamma

$\underset{\substack{\text { INCH } \\ \text { Caliper }}}{ }$

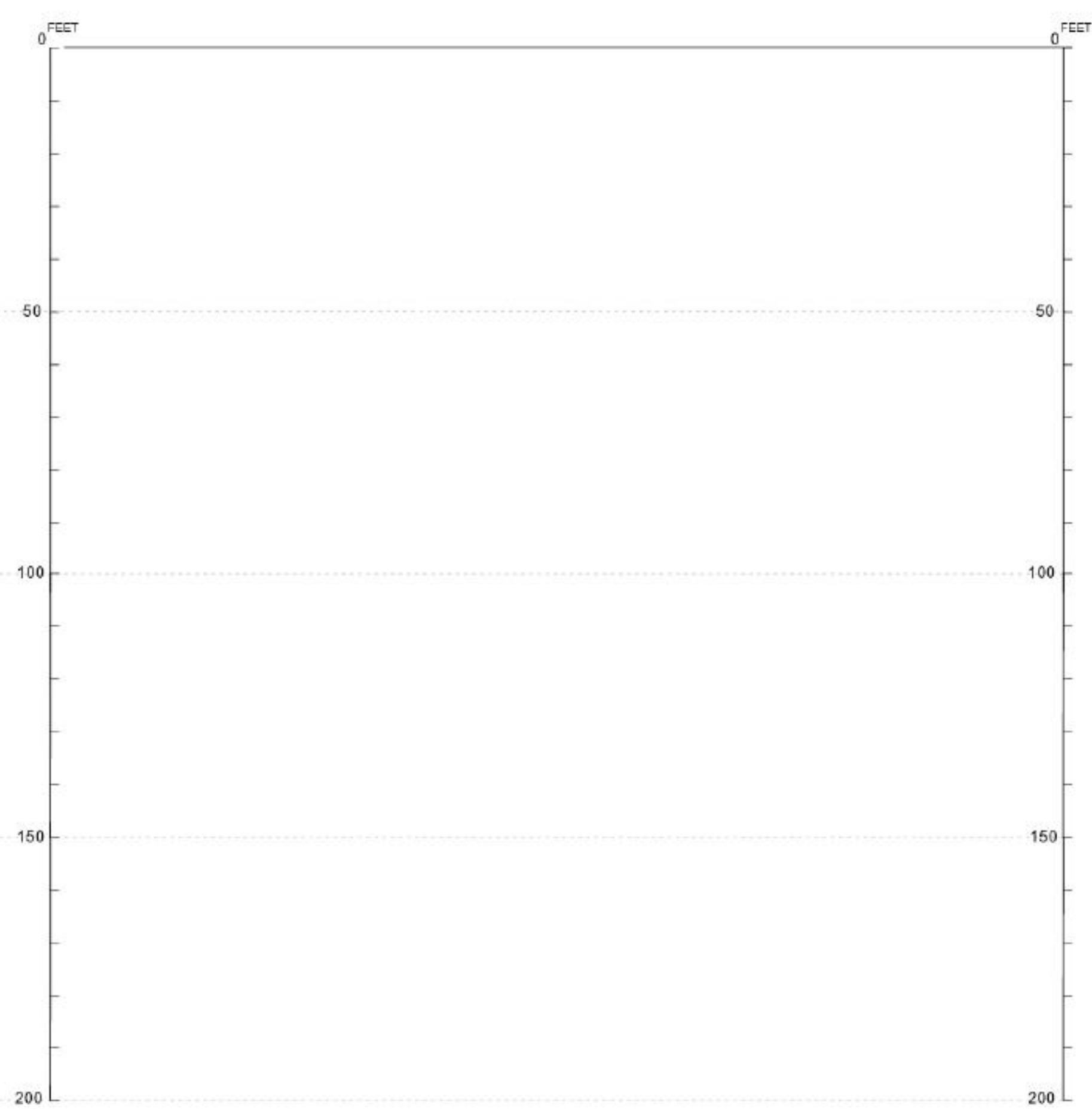



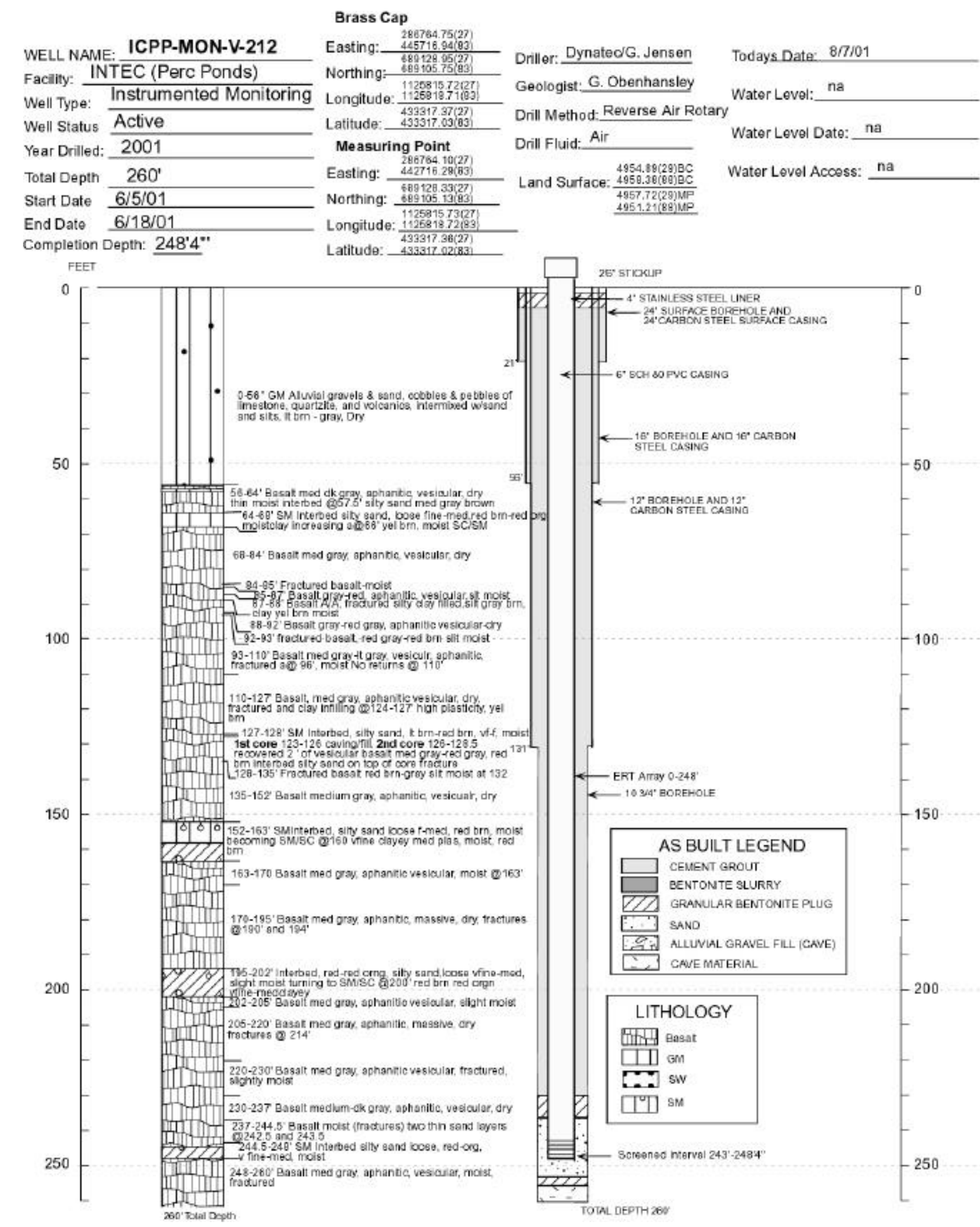
$\begin{array}{r}4957.72(293) M P \\ 4.951 .21(89) M P \\ \hline\end{array}$
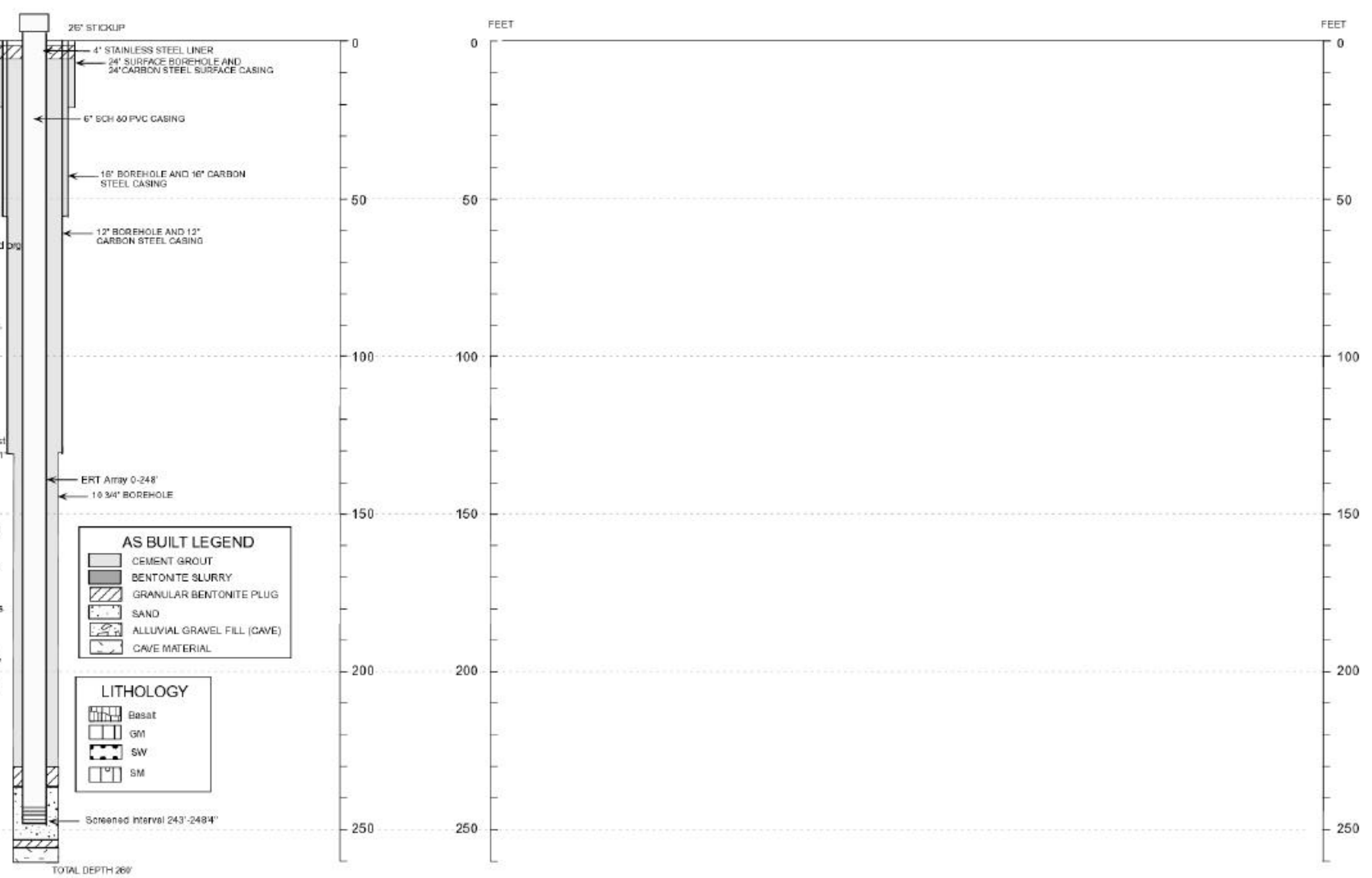
WELL NAME: ICPP-SCI-V-213 (AA) Brass Cap

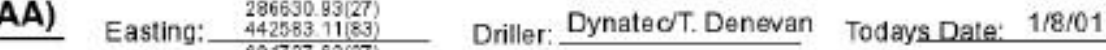

Facilty:

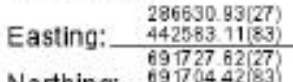

Well Type: Sclentific Instrumentation

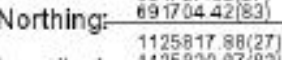

Well Status Active

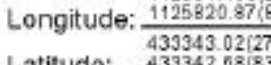

Year Drilled 2000 Measuring Point

G. Obenhanslay

Total Depth $560^{\circ}$

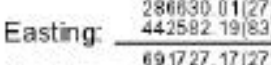

Drill Method: Coring

Water Level:-

Start Date $\frac{8 / 29 / 00}{9 / 18 / 00}$

Drill Fluid: Water w/polymer Water Level Date: na

End Date $\frac{9 / 18 / 00}{\text { Completion Depth: } 495}$

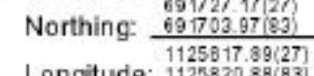

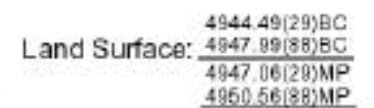

mpletion Depth: $495.5^{\prime}$
FET

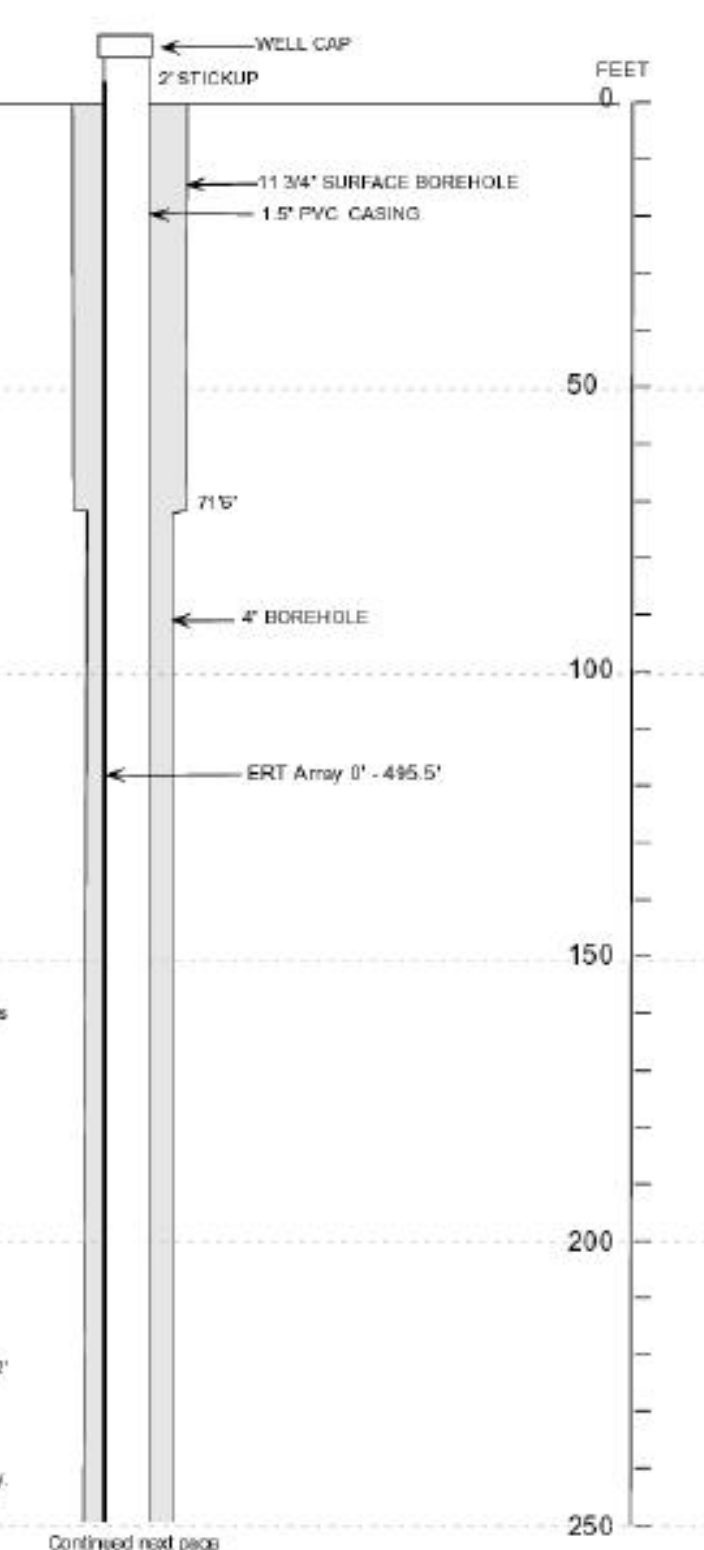

50

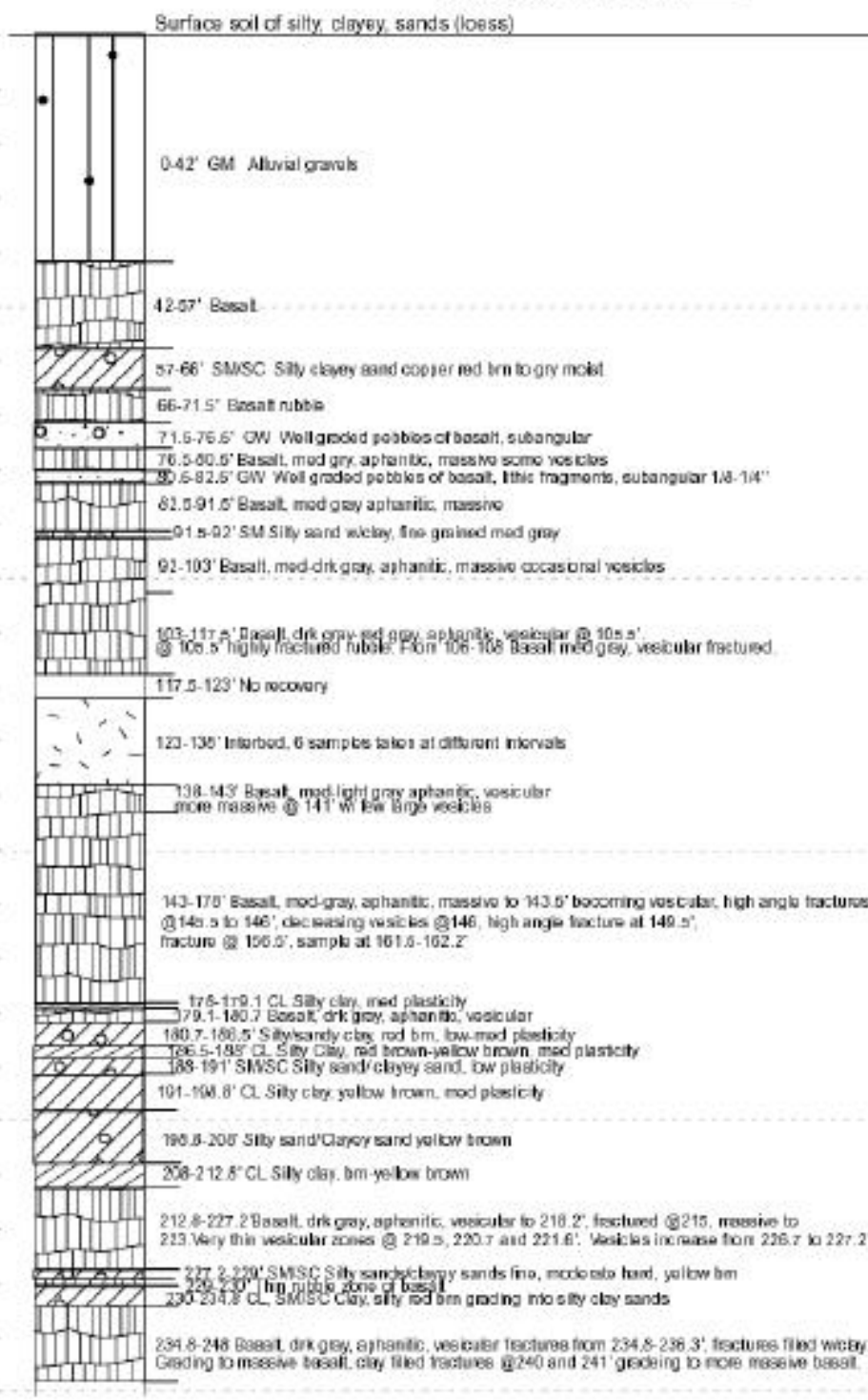


WELL NAME: ICPP-SCI-V-213 page 2 Easting

NTEC (Perc Ponds)

Well Type:

Well Status Activ

Northing:-

Driller: DynatecrT. Denevan Todas Dan 1/810

Year Drilled: 2000 Longitude: Goologist:G. Obenhansley Drill Method:Coring

Total Depth $\frac{560^{\prime}}{8 / 29 / 00}$

End Date $9 / 18 / 00$

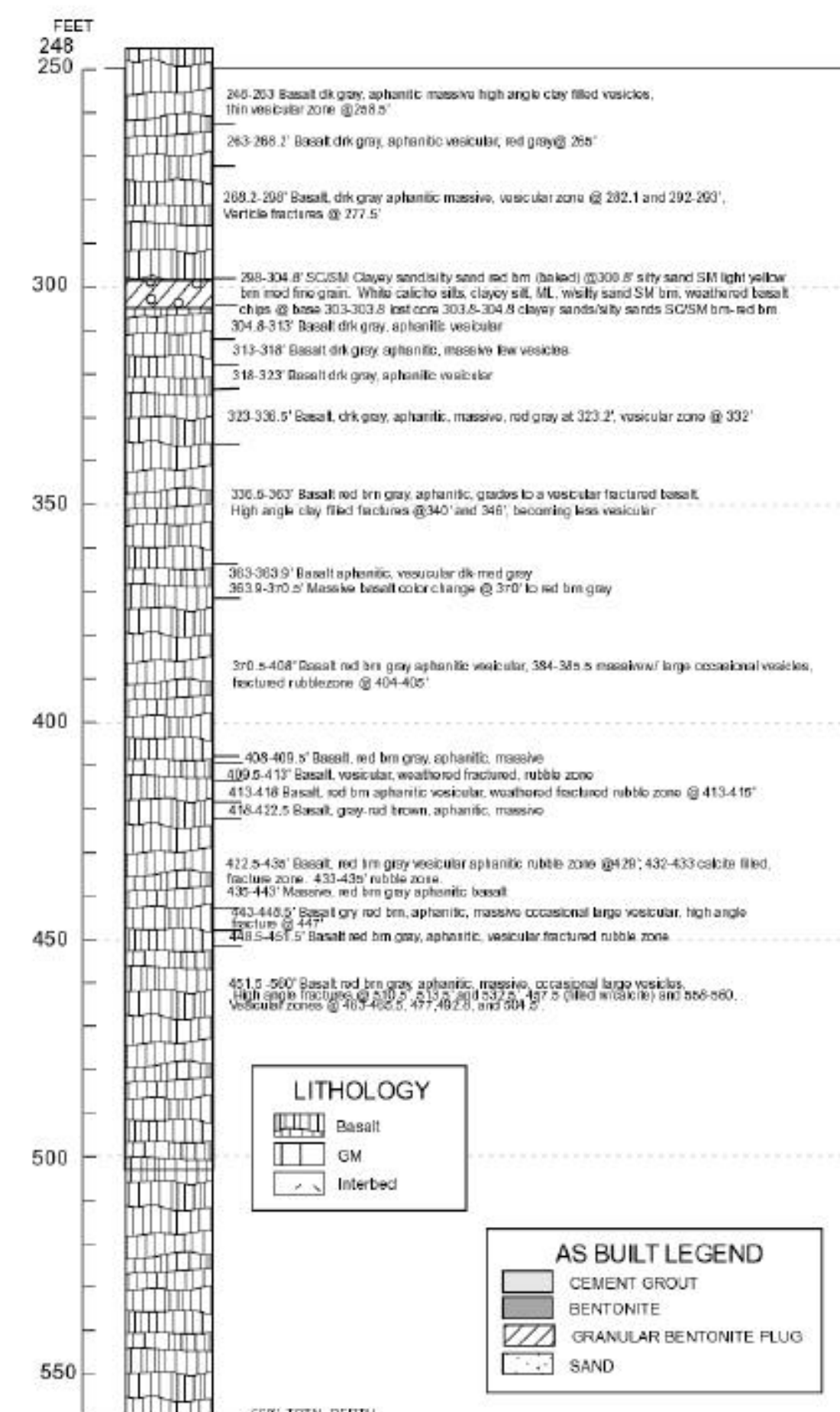

wer watertovado na Completion Depth: $495.5^{\prime}$ Land Surface:

Water Level Accoss. na $\underset{\substack{n \\ \text { APIN }}}{\text { Neutron }}$ APl-GR
$\underset{\mathrm{CFs}}{\text { Gamma }}$

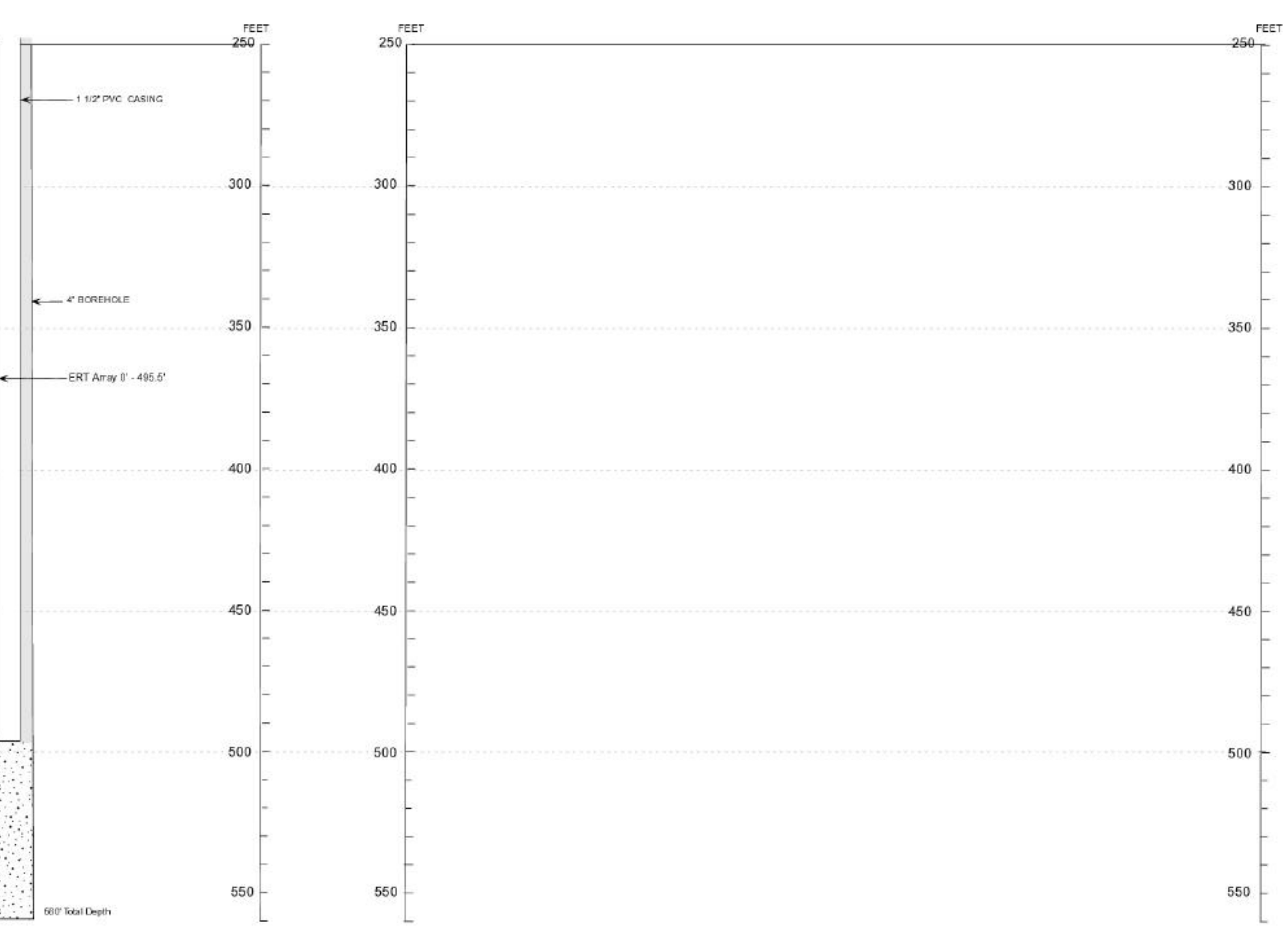


ICPP-SCI-Y-214

WELL NAME- (ICPP\#186)

Facility: INTEC (Perc Ponds)

Brass Cap

Driller: USGS

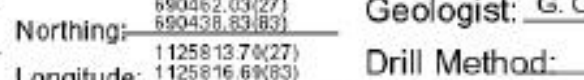

Todays Date:

Well Type:Instrumented borehole

Year Drilled2001

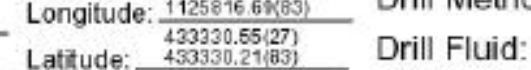

Total Depth 500' Latitude: 433330,21
Measuring Point

Start Date

Drill Fluid.

Water Level:

Water Level Date: na

End Date

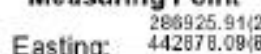

Water Level Access: na Longitude: 112588167

Completion Depth: $443^{\prime}$

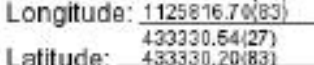

FEET 1.56' GM Allumid roravels

$50-$

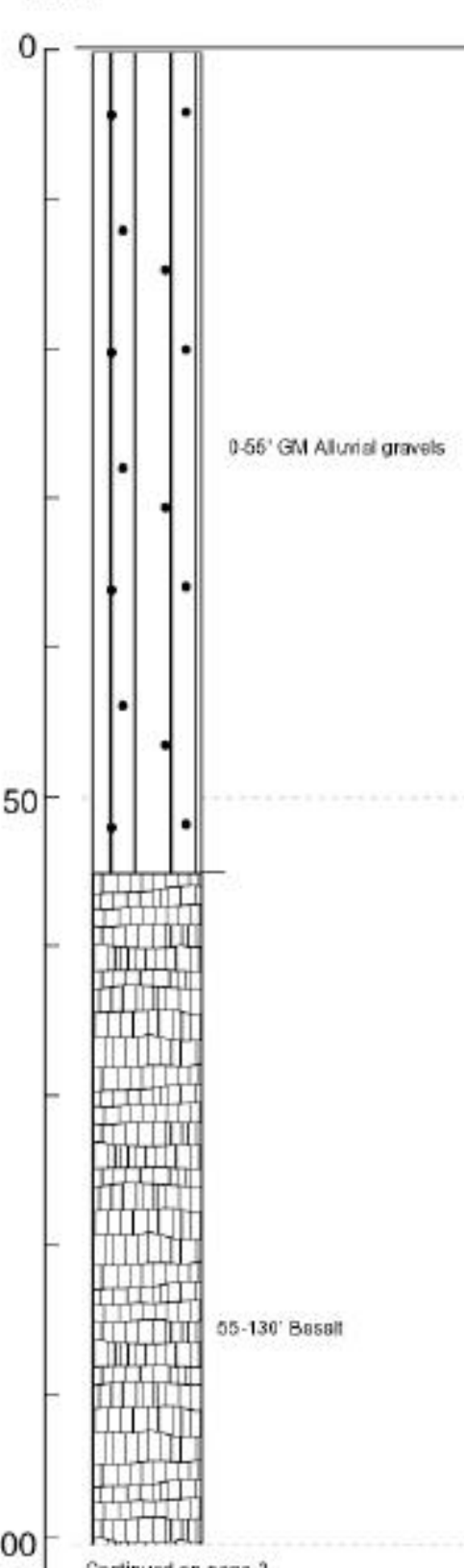

100 Continued on pega

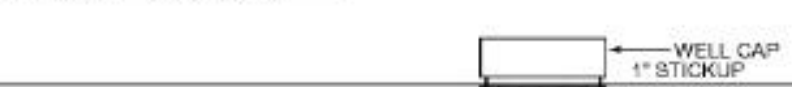

FEET

FEET $\underset{\text { API-N }}{\text { Neutron }}$

Gamma Gamma

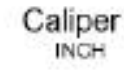


ICPP-SCI-V-214 pg 2

WELL NAME: (CPPAC)

Facility INTEC (Perc Ponds)

Well Type: Instrumented

Well Statusctive

Year Drilled2001

Total Depth 175'4"

tart Date $4 / 1100$

Completion Depth: 174'3'

FEET

Brass Cap

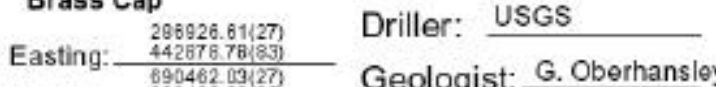

Geologist: G. Oberhans

Drill Method

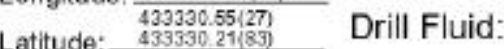

Measuring Point

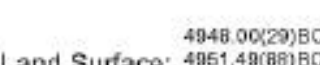

Water Level- na

Water Level Date: na

Water Level Access:na

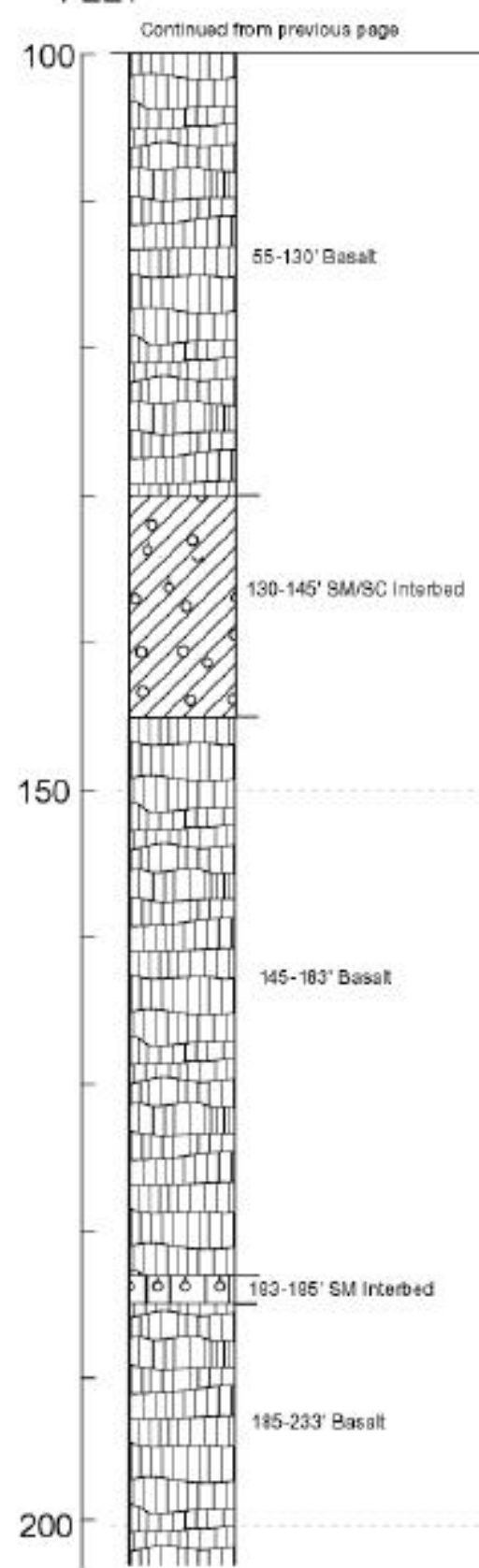

Easting: 4442876.09810

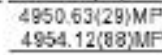
Longitude

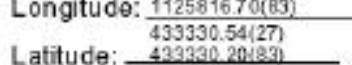

FEET

Contenves tom preavuus pros

FEET

100

$\underset{\text { Natur-GR }}{\text { Natural Gamma }}$

$\underset{\text { APINN }}{\text { Neutron }}$

$\underset{\mathrm{CP3}}{\text { Gamma Gamma }}$

$\underset{\substack{\text { INCH } \\ \text { Caliper }}}{-}$

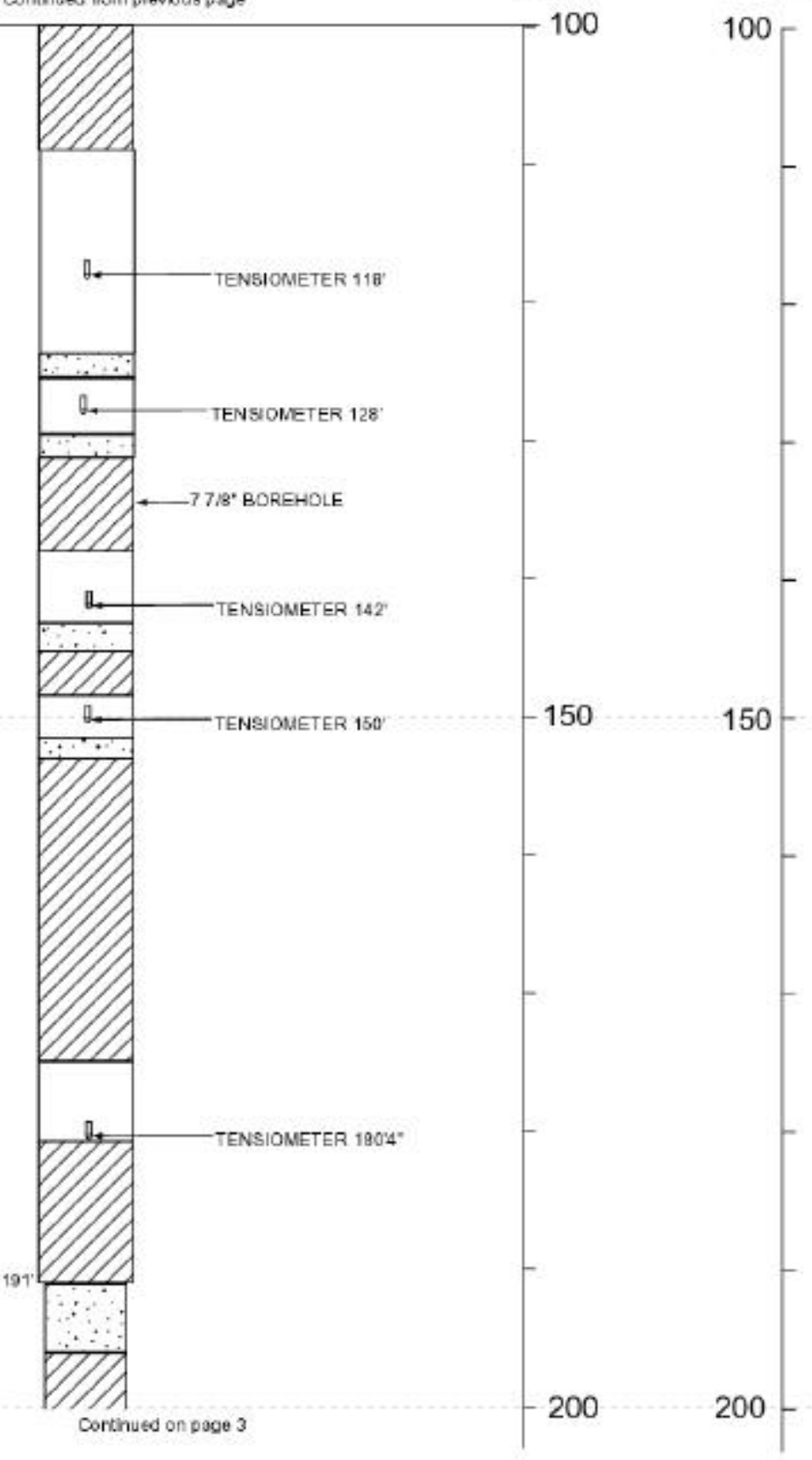


ICPP-SCI-V-214 pg 3

WELL NAME: (ICPPH186)

Facility: INTEC (Perc Ponds)

Well Type: Instrumented

Well Statusctive

Year Drilled2001

Total Depth $175^{\prime} 4^{\prime \prime}$

Start Date $4 / 02 / 00$

Completion Depth: $174^{\prime} 3^{*}$

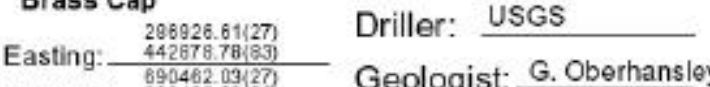

Geologist: G. Oberhans

Drill Method

Drill Fluid:

Measuring Point

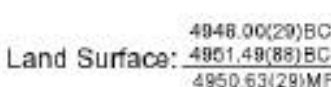

$4950.632929 \mathrm{MP}$ Northing: 6.690464 .39 .13 Longitude:

FEET

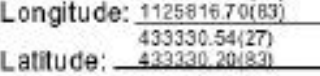
Contrused trom prevous pron 200

FEET
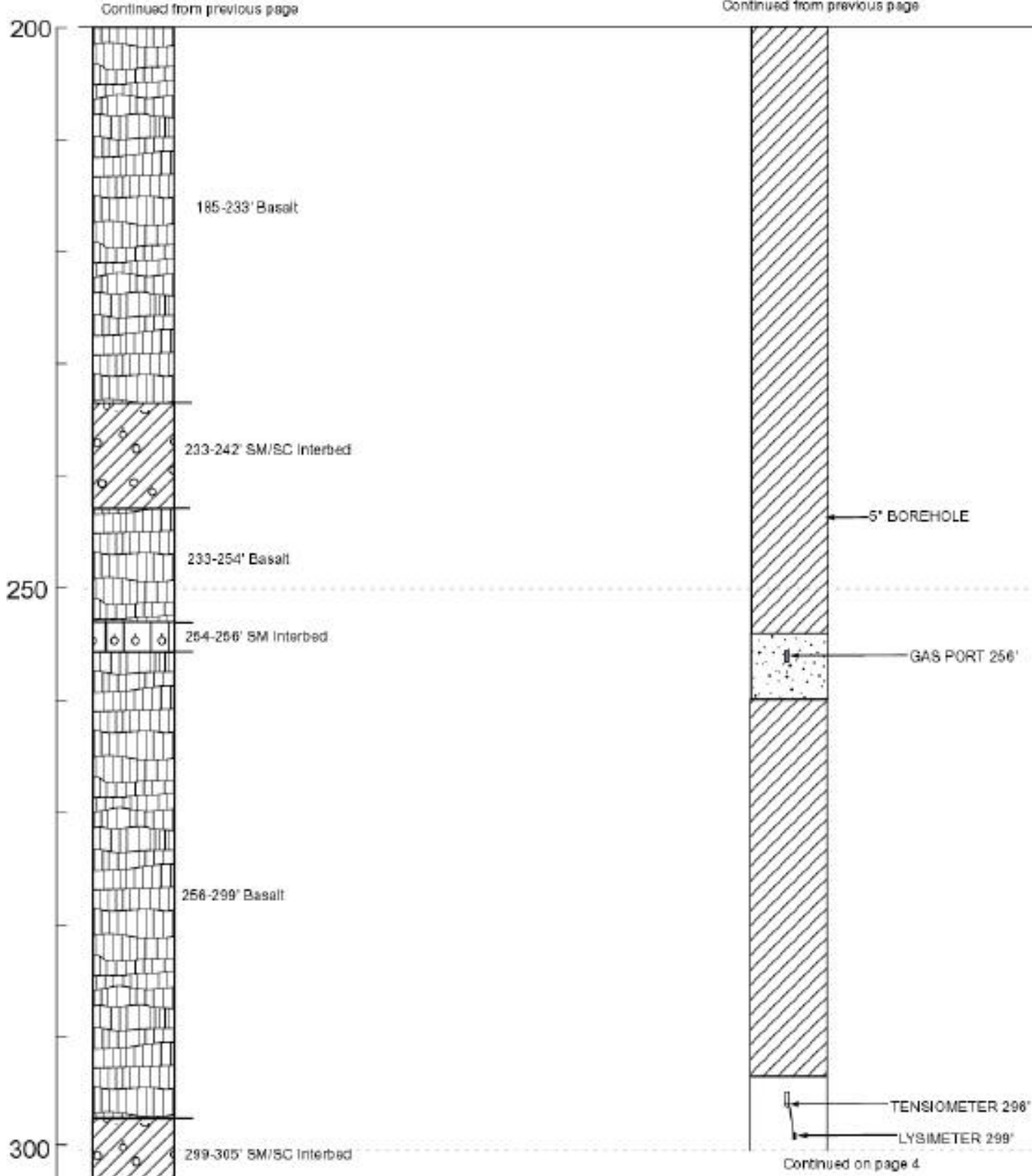

250
Todays Date: 8/1/10

Water Level: na

Water Level Date: $n$

Water Level Access:

$\underset{\text { APl-GR }}{\text { Natural Gamma }}$ 
ICPP.SCI-V-214 pg 4

WELL NAME (ICPA

Facility: NTEC (Perc Ponds)

Well Type: Instrumented

Well Statusctive

Year Drilled2001

Total Depth $175^{\prime} 4^{\prime \prime}$

Start Date $4 / 0200$

Completion Depth: $174^{\prime} 3^{*}$

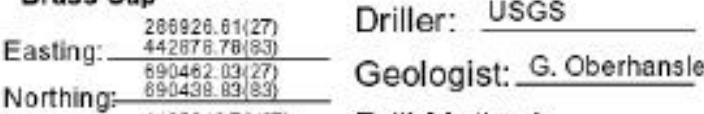
Northin

Drill Metho

Drill Fluid:

Measuring Point

4948.002538

Easting. $42050.63129 \mathrm{MP}$

Water Level: na

Level Date: na

Water Level Access:

FEET

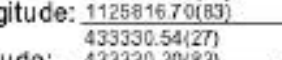

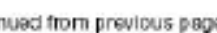

Conthued trom prevous pso

FEET

${ }^{300}$ W

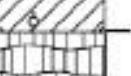

IIIm

페

(intin

(in!

350

\begin{tabular}{|c|}
\hline-1 \\
\hline-1 \\
\hline-1 \\
\hline-1 \\
\hline
\end{tabular}

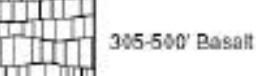

III

mind

IIIIIII

(i)

$400-$

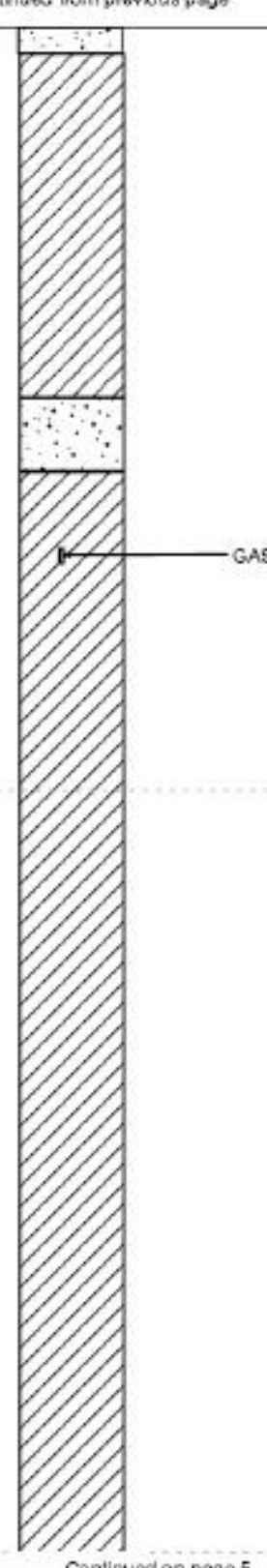

contiruesion pase 5
Natural Gamma

INCH

FEET

300 
ICPP-SCI-V-214 pg 5

WELL NAME:(ICPPA

Facility: INTEC (Perc Ponds)

Well Type: Instrumented

Well Statusctive

Year Drilled:2001

Total Depth $175^{\prime \prime} 4^{\prime \prime}$

End Date 4/11/00

Completion Depth: $174^{\prime} 3^{\prime \prime}$

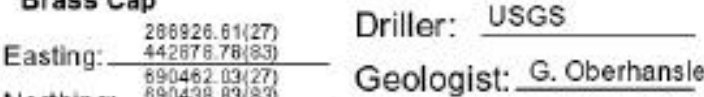

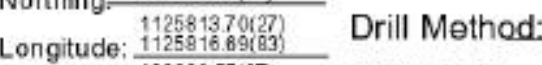

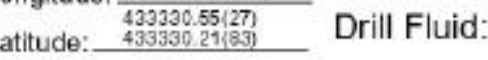
Measuring Point

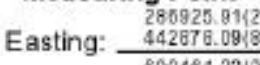

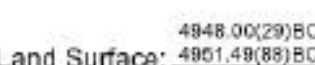

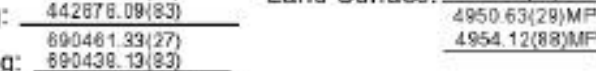
Longitude:

FEET

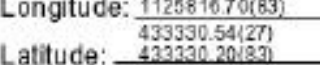
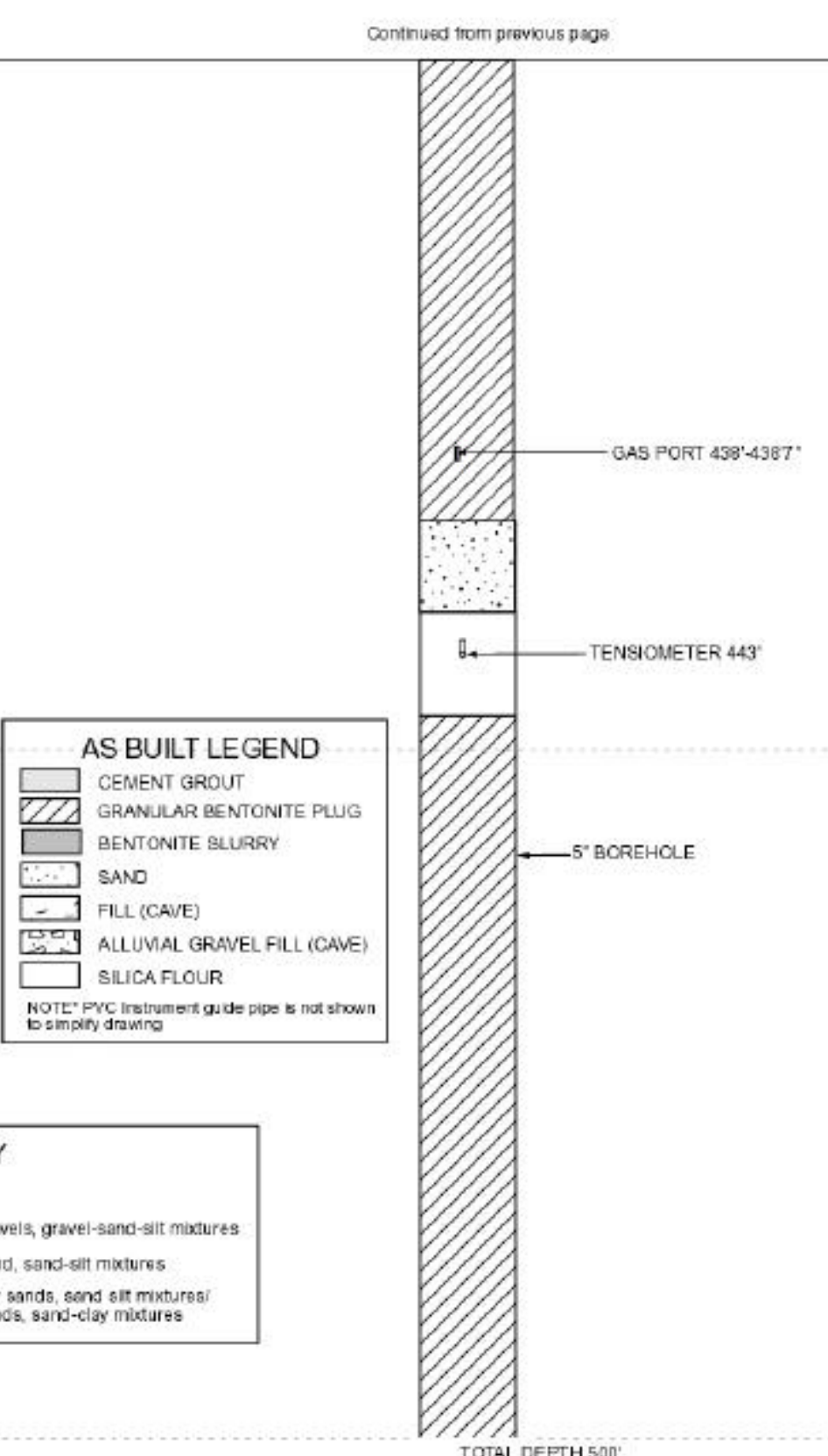
Water Level: na

Water Level Date: na

Water Level Access:na

TTAL DEFTH $500^{\circ}$

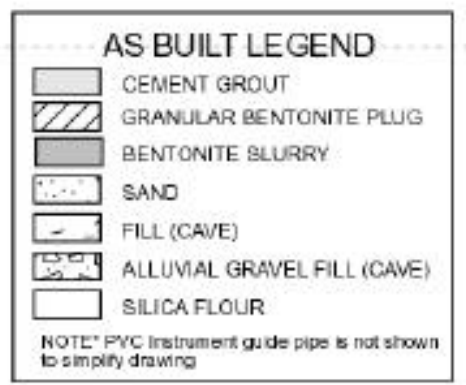

LITHOLOGY

面整 Bessot

T. 5 sil

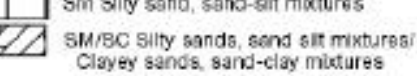

450

- intint
FEET

FEET

400

$\underset{\text { Natur-GR }}{\text { Namma }}$

$\underset{\text { APIN N }}{\text { Neutron }}$

$\underset{\mathrm{CPB}}{\operatorname{Gamma}}$

$\underset{\text { Caliper }}{\text { INCH }}$ 
ICPP-SCI-Y-215

WELL NAME. (ICPPHAS)

acility: NIEC (Perc Ponds)

Brass Cap

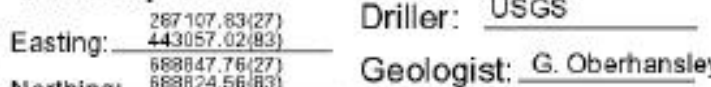

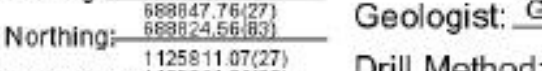

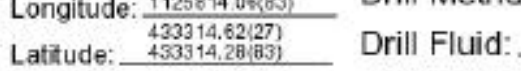

Well Status Active

Total Depth 504.3

Me asuring Point

Start Date

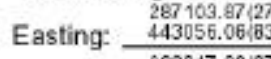

Drill Fluid:-

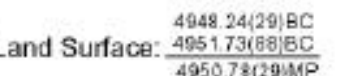

Longitude: $\frac{11258719}{1257.07}$

Completion Depth: 482'

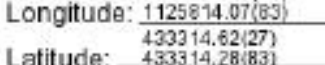

FEET

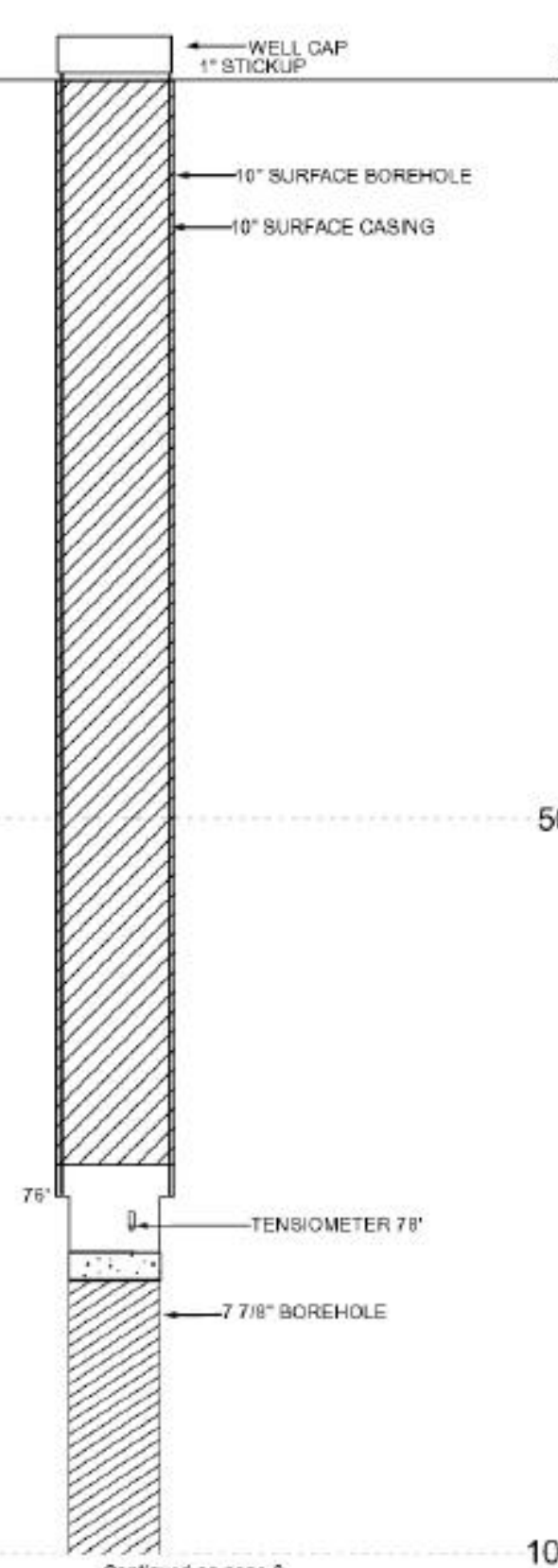

100

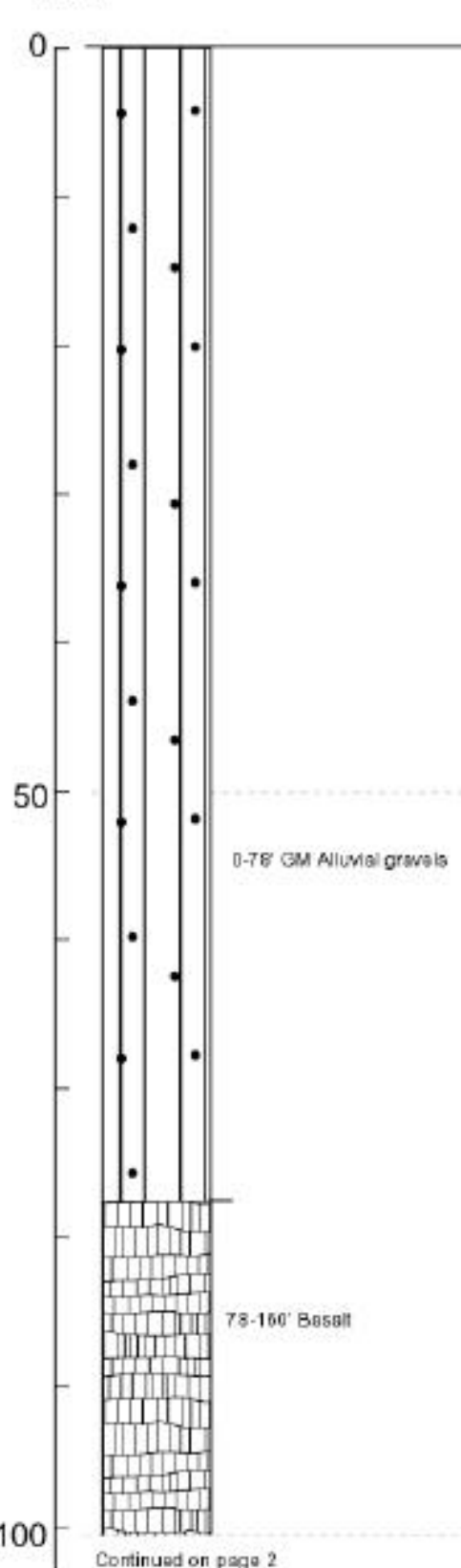

Continuod on pega 2
Todays Date:

Water Level: na

Water Level Date: na

Water Level Access:na

FEET

FEET

50

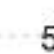

100

100

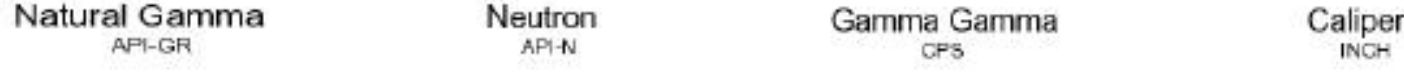

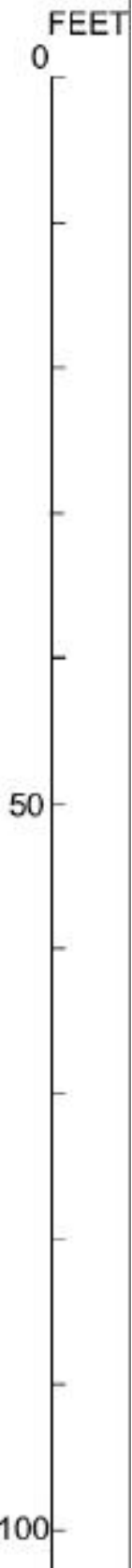


ICPP-SCI.V-215pg 2

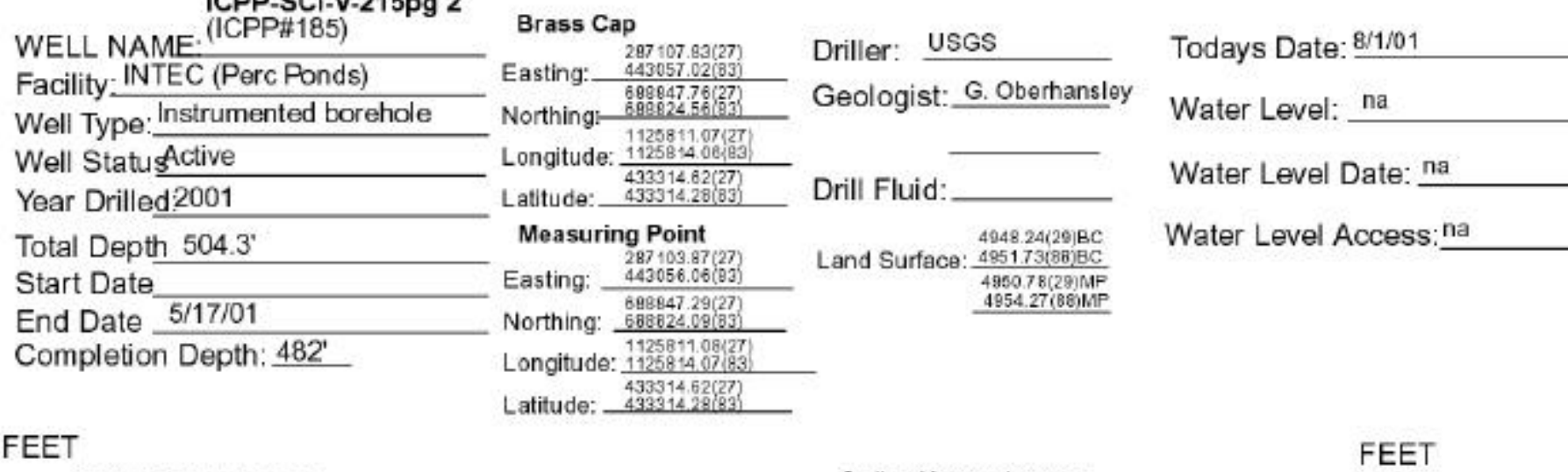

FEE

Continued trom previous page

$-100$

FEET
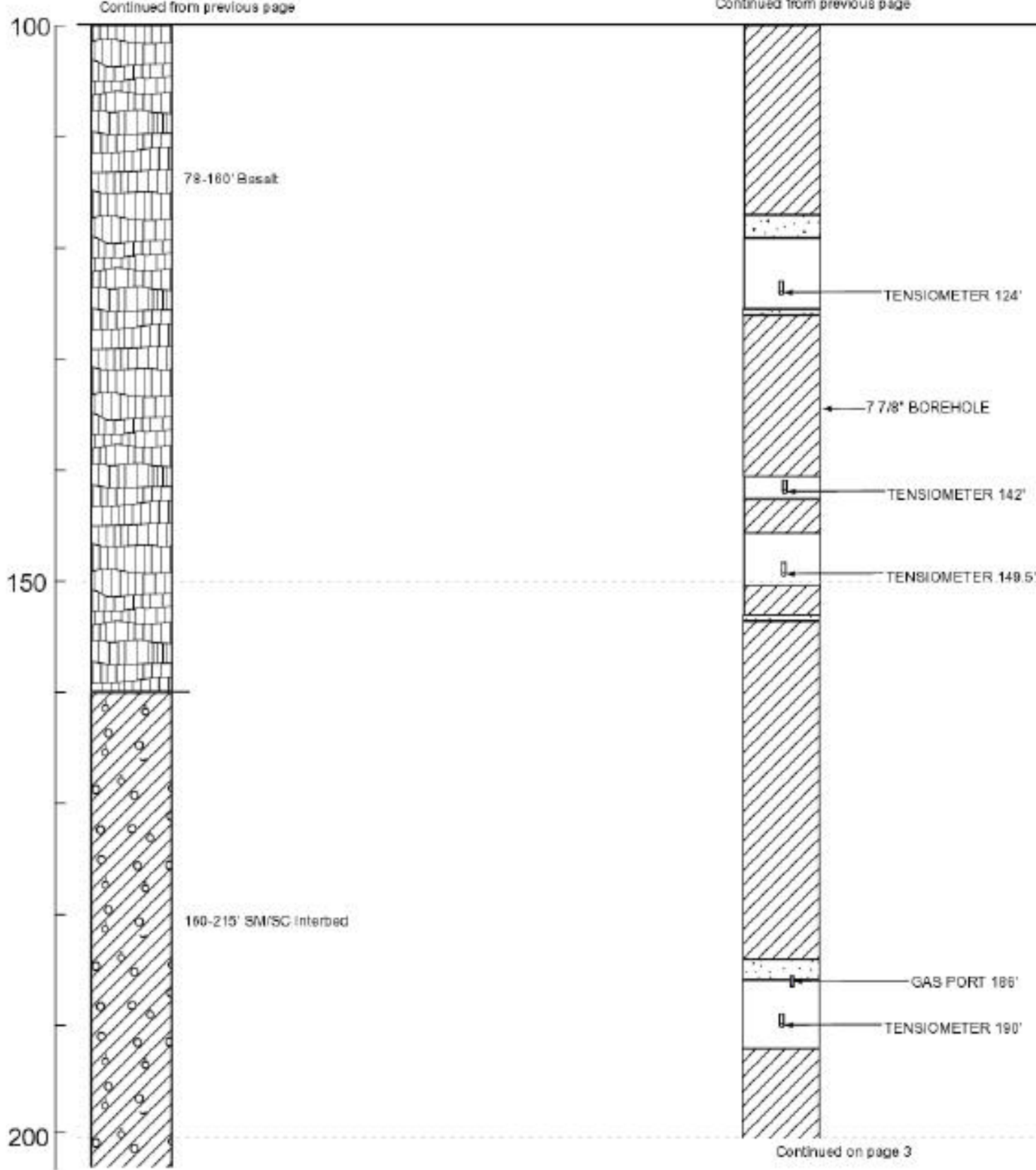

100

Natural Gamm

Neutron

Gamma Gamma

Caliper
INCH 
ICPP-SCI-V.-215 pg3

Brass Cap

acility : INTEC (Perc Ponds)

Well Type: Instrumented borehole

Well Status Active Northin

Driller: USGS

Geologist: G. Oberhans

Year Drilled2001

Total Depth 504.

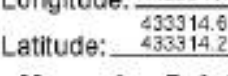
Drill Fluid:

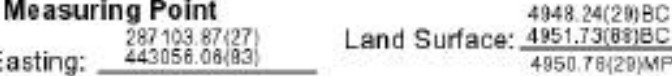

Start Date $5 / 17 / 0$

Northing: $\frac{686824.098 .6}{1125811.08}$

Longitude: $112585140727(63)$

180-215' swsc intertod

250

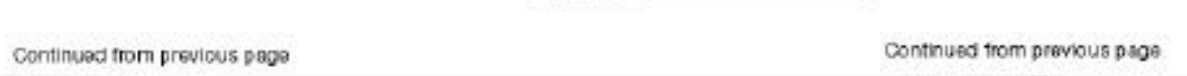

FEET

Water Level Date: na

Water Level Access:

FEET

$\underset{\text { Natural Gamma }}{\text { APl-GR }}$

$\underset{\text { Neutron }}{\text { AFI-N }}$

Gamma Gamma

$\underset{\substack{\text { Caliper } \\ \text { INCH }}}{ }$

200
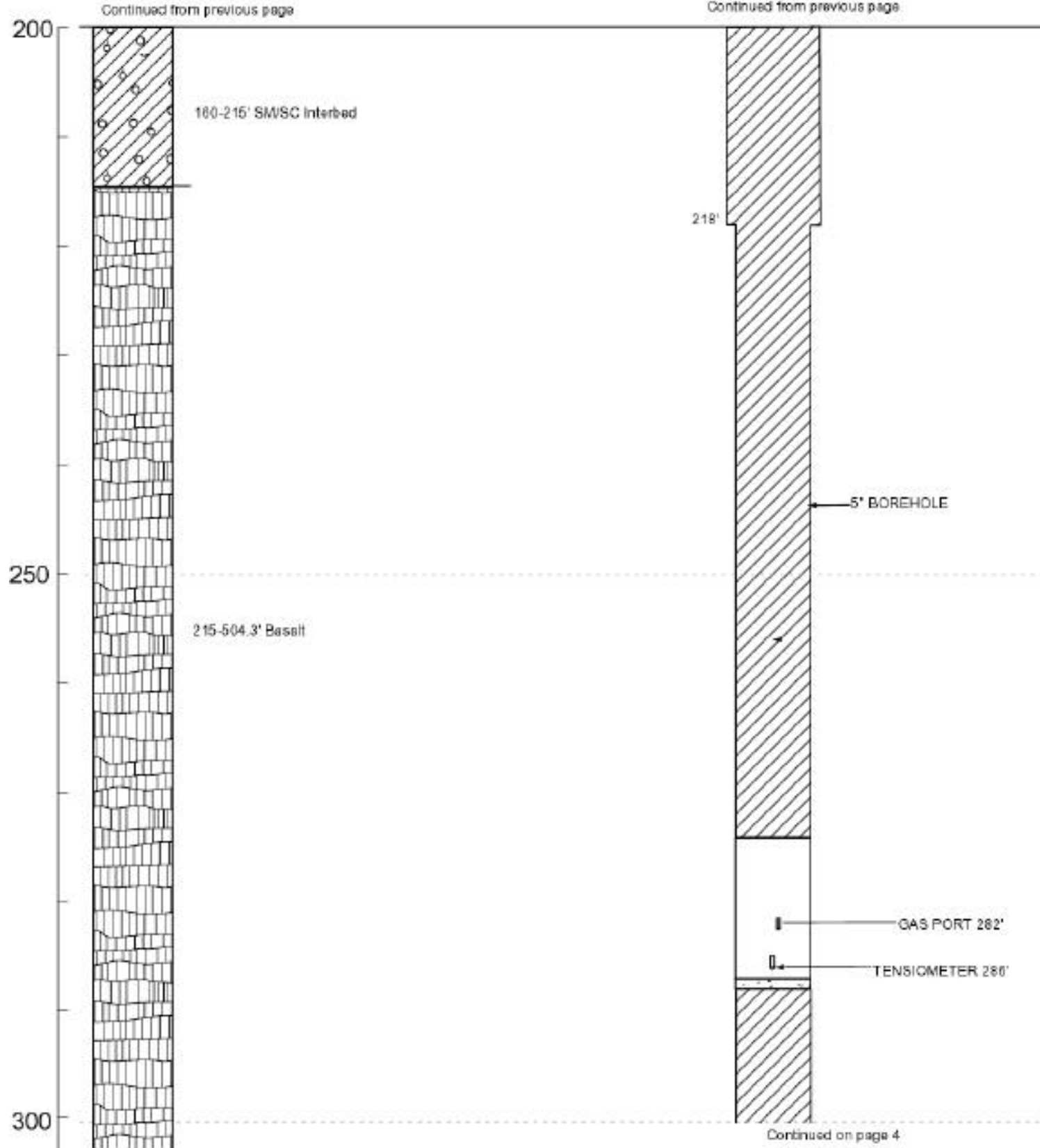
ICPP-SCI-V-215 pg4

WELL NAME: (ICPPH Fl

287107.85[27) Driller: USGS

Well Type Instrumented borehole

Well Statusctive

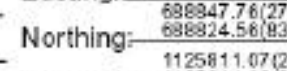

eologist: G. Oberhansle

Water Level: na

Total Depth 504 Drill Fluid:

Total Depth 504.3 Measuring Point

4948.24(29)8C Water Level Access:

End Date 5/17/01

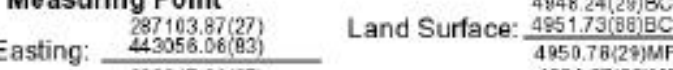

Completion Depth: $482^{\prime}$

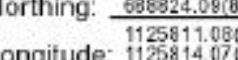

FEET

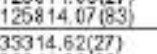

$300-$ Continuod tom previlous 8000

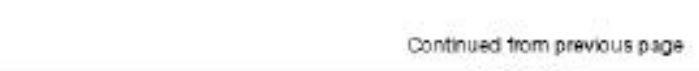

FEET

FEET

Natural Gamma

$\underset{\text { Neutron }}{\text { APIN }}$

$\underset{\mathrm{CPS}}{\operatorname{Gamma}}$

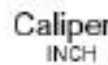

350

400

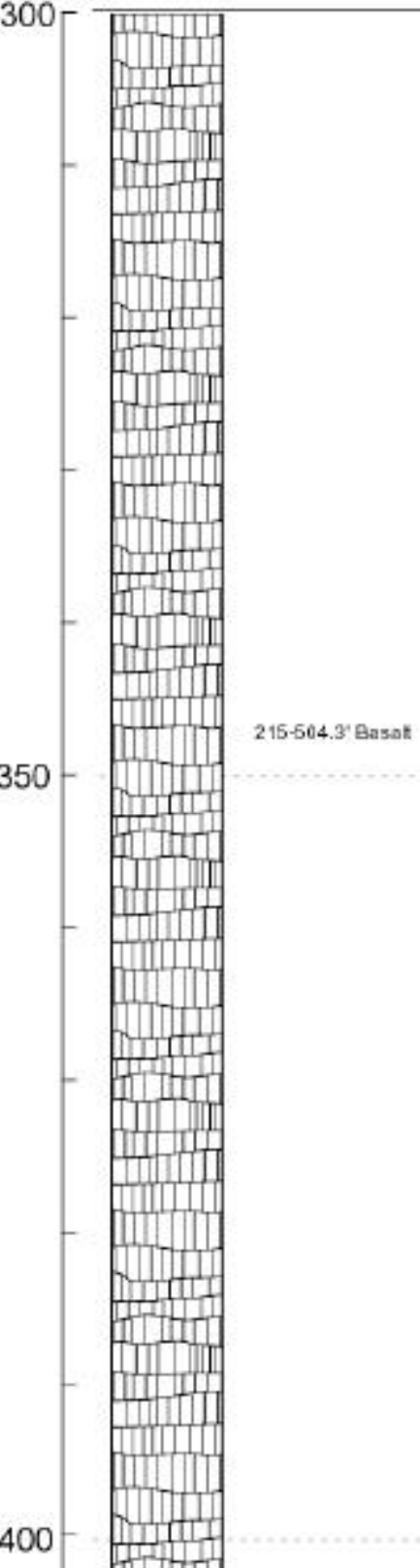

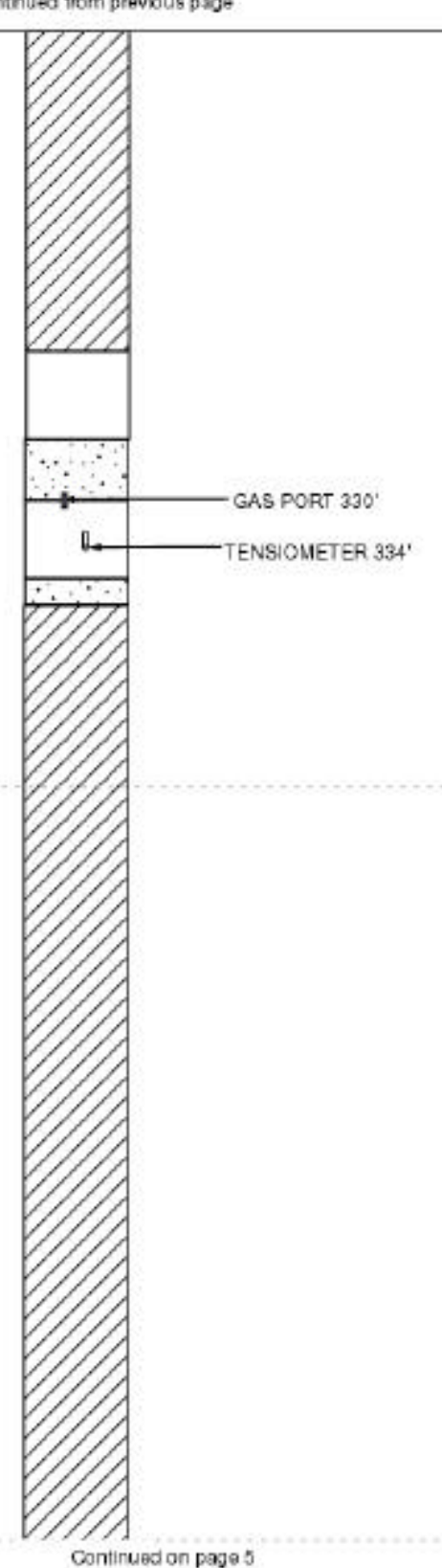

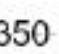

400
35

300

400 
ICPP-SCI-V-215 pg5

Brass Cap

WELL NAME: (CPPHSS)

Facility NIEC (Perc Ponds)

Brass $\mathrm{Cap}$

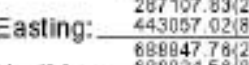

iller: USGS

Well Statusctive

Year Drilled2001

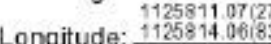

Total Depth 504.3' Longitude: $\frac{1}{4359314.625}$

Drill Fluid

Me asuring Poin 28703

Water Level Access:

End Date 5/17/01

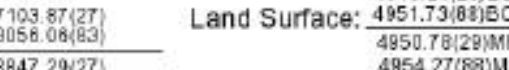

Completion Depth: 482

Northing: $\frac{68892409}{112581108}$

Contrinued trom previlus 8908

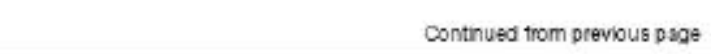

Continued trom previous page
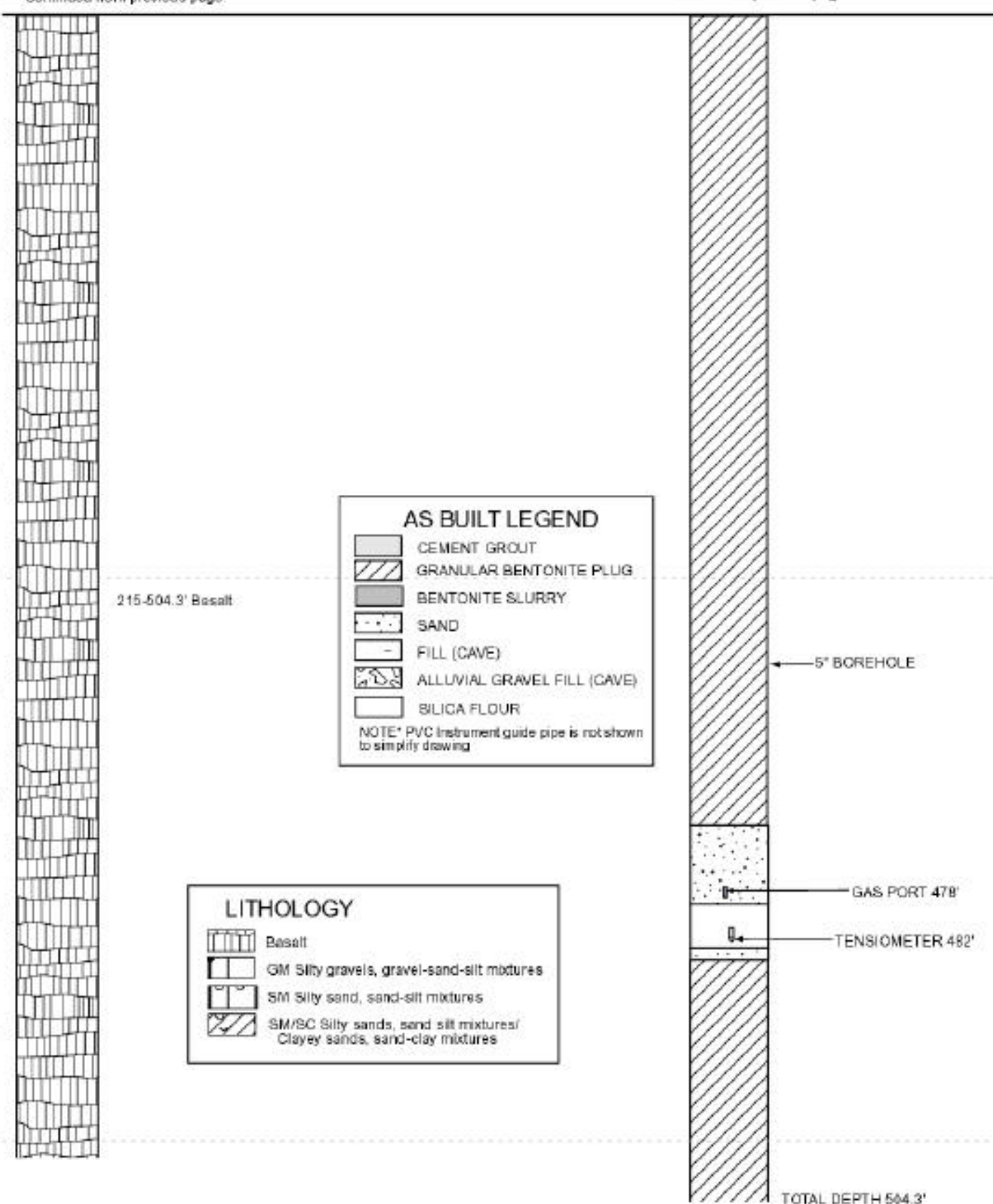

$\underset{\text { NAP-GR }}{\text { Natural Gamma }}$

Neutron

Gamma Gamma

$\underset{\substack{\text { Caliper } \\ \text { INCH }}}{ }$
450

500

FEET 


\section{Appendix B \\ Letter Report - Perched Water}


B-2 


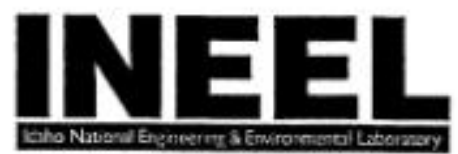

\section{INTEROFFICE MEMORANDUM}

\begin{tabular}{|c|c|c|c|}
\hline Date: & March 7, 2001 & & \\
\hline To: & Michael Lowis & MS 4110 & $6-0623$ \\
\hline From: & $\begin{array}{l}\text { S. L. Ansley S4A } \\
\text { L. C. Hull }\end{array}$ & $\begin{array}{l}\text { MS } 2107 \\
\text { MS } 2107\end{array}$ & $\begin{array}{l}6-0945 \\
6-1922\end{array}$ \\
\hline Subject: & $\begin{array}{l}\text { EVALUATION OF PER } \\
\text { DISCHARGE FACILIT }\end{array}$ & CEC WAS & \\
\hline
\end{tabular}

This letter report presents the compilation and evaluation of data in support of the DOE/ID response to the State of Idaho draft permit for the INTEC Wastewater Discharge Facility. Compliance monitoring at the perched water does not provide an adequate measure of impacts to the Snake River Plain Aquifer. The State of Idaho Handbook for Land Application of Municipal and Industrial Wastewater (1996) states that "ground water compliance monitoring points must be established at locations most likely to provide a reliable and representative measure of the compliance of a wastewater-land application treatment system with the water quality standards and the WLAP permit conditions". The Snake River Plain aquifer is the water resource that we are trying to protect. Measurement of the impact of the ponds on the Snake River Plain aquifer, and requiring compliance in the Snake River Plain aquifer, is the way to assure that the resource is protected for future beneficial uses.

Any man-made perched water bodies associated with the percolation ponds are dependent upon the volume of water discharged to the ponds. In order to be available for beneficial use, a water body must be continually saturated and productive. Pond associated-perched water will gradually drain away when discharges to the ponds are ended, and will therefore not be available for beneficial use. In addition, perched water quality immediately adjacent to the pond in the surficial alluvium and shallow perched water zones will rapidly match effluent quality making perched water monitoring redundant to effluent monitoring. The regulations do not give the State the authority (with very minor exceptions) to regulate discharge to the pond. Therefore, the State may not have the authority to require monitoring of the perched water in the immediate vicinity of the pond.

Natural perched water capable of exploitation for beneficial use beneath the new percolation ponds is associated with flow in the'Big Lost River. When the river is flowing, there are shallow perched water bodies within the alluvium and at depths near $150-170 \mathrm{ft}$ bls in basalt. Without flow in the channel, the perched water begins to drain. As observed, within 6 months of the last recorded flow, the upper $170 \mathrm{ft}$ of the vadose zone is drained. Therefore, shallow perched water bodies are a very intermittent phenomenon and do not provide a reliable resource for beneficial use. After 9 months, water persists at a depth of $300 \mathrm{ft}$ bls. This deeper perched water may persist for longer time periods, but probably does not represent a viable water supply for.heneficial use. 
Michael Lewis

March 7, 2001

Page 2

When aquifer monitoring wells were drilled in the Spring of 2000, copious quantities of water entered boreholes near the Big Lost River channel at various depths. Downhole video logs show water cascading from the borehole walls at 170,198 , and $235 \mathrm{ft}$ bls. In ICPP-MON-A-164B, the quantity of water entering the hole was so great that the video picture was obscured (March 2000). Drilling to the 150-170 ft interbed in the fall and winter of 2000-2001 (flow in the Big Lost River channel has been zero since the end of May 2000) found much smaller quantities of water. In January 2001, perched water was detected in two monitoring wells completed within this zone. When these two wells were measured a month later, the wells were dry.

Perched water, whether derived from the Big Lost River or the percolation ponds, migrates downward to the Snake River Plain Aquifer. Water added to the vadose zone from the ponds or from the Big Lost River, will migrate to the aquifer long before the time that the INEEL is released for unrestricted use. A future land owner attempting to use perched water for a beneficial use would be using water that entered the vadose zone from future flow in the Big Lost River channel, not from the ponds. Water in the vadose zone during pond operations, which the State proposes to monitor for compliance, has no possible future beneficial use in the vadose zone. Only after it has migrated to the aquifer, will it be available for beneficial use.

For clarification, definitions of beneficial use, beneficial uses of groundwater, and waters of the State are provided below.

Beneficial use:

Source: State of Idaho Handbook for the Land Application of Waste Water

Definition: "Any of the various uses which may be made of the water of Idaho, including. but not limited to, domestic water supplies, industrial water supplies, agricultural water supplies, navigation, recreation in and on the water, wildlife habitat, and aesthetics. The beneficial use is dependent upon actual use, the ability of the water to support a nonexisting use either now or in the future, and its likelihood of being used in a given manner. The use of water for the purpose of wastewater dilution or as a receiving water for a waste treatment facility effluent is not a beneficial use."

Beneficial uses of ground water:

Source: State of Idaho Handbook for the Land Application of Waste Water Definition: " Various uses of ground water in Idaho including, but not limited to, domestic water supplies, industrial water supplies, agricultural water supplies, aquacultural supplies and mining. A beneficial use is defined by actual current uses or future uses of the ground water."

Waters of the State of Idaho:

Source: Idaho Department of Water Resources

Definition: "all surface or ground water located within the boundaries of Idaho or in boundary streams, rivers, and lakes." Note: The focus of this definition is that it includes all water within the State that has not yet been diverted into a well or a diversion system for use by a valid water right (G. Sehlke). 
Michael Lewis

March 7, 2001

Page 3

\section{Recommendations}

Recommendations for responding to the State of Idaho conditional draft permit for the new percolation ponds are:

1) groundwater compliance monitoring points for the INEEL Wastewater Discharge Facility should be in the Snake River Plain Aquifer in wells ICPP-MON-A-165 and ICPP-MON-A166 , downgradient of the facility boundary with upgradient background data obtained from ICPP-MON-A-167B;

2) perched water monitoring for observation only could be a condition of the permit for the new ponds as a compromise.

If the State insists on compliance monitoring of the perched water, then some rationale or justification would have to be provided by them for which zone represented water with a potential future beneficial use. After the pond begins operating, there will likely be perched water zones forming in the surficial alluvium, at the 150-180-ft depth, and around $300 \mathrm{ft}$ bls. However, at depths less than $200 \mathrm{ft}$, perched water does not carry over from year to year and so does not provide a reliable water supply for beneficial use. Only at depths of $300 \mathrm{ft}$ is perched water present for at least 9 months after flow ceases in the Big Lost River.

Observational monitoring of the perched water may be presented as an alternative to compliance monitoring. In general, we do not recommend collecting data that do not support decision making. However, attaching a value other than compliance to such data may provide a negotiating point with the State. Information to be gained from monitoring the perched water zone(s) includes: 1) demonstrating the attenuation of chemicals discharged to the pond as they infiltrate to the aquifer; 2) demonstrating that infiltrate does not leach contaminants from the vadose zone; 3 ) measuring contaminant build-up from wastewater infiltration; and 4) measuring contaminant migration rates. All vadose zone wells could be instrumented for water level monitoring beginning with the start-up of discharge to the ponds. A periodic monitoring program (quarterly?) would sample and analyze standing water in the monitoring wells during the first two years of operation. If the perched water zones match the effluent to the pond, and changing conditions are not observed, then this quaterly monitoring could be discontinued or the frequency reduced. 
Michael Lewis

March 7, 2001

Page 4

\section{Supporting Data}

The following paragraphs contain supporting data regarding the issue of perched water beneath the new percolation ponds. Much of the stratigraphic and perched water information was provided by wells drilled for the INEEL Vadose Zone Research Park. Big Lost River flow data were obtained from the USGS Water Resources Division in Idaho Falls.

The INEEL Vadose Zone Research Park encompasses the location of the new percolation ponds and is coordinated by the INEEL Subsurface Science Program, the Manager of Environmental Monitoring, and the Geosciences organization. This effort supports the role of the INEEL as the lead laboratory for vadose zone science. Current funding for the park is supporting the installation of approximately 26 wells; each designed as a monitoring well, an instrumented borehole, or a geophysical borehole. Drilling is ongoing and scheduled for completion during the summer of 2001. The information obtained during the drilling, logging, and installation of these wells, in addition to four aquifer wells installed in 2000 , provides valuable information on the stratigraphy and hydrology beneath the new facility. Figure A shows the locations of vadoze zone wells associated with the research park and aquifer wells constructed to collect background water quality data. Table A summarizes the wells completed to date and Attachment A contains well diagrams and lithologic logs.

The closest USGS flow monitoring stations along the Big Lost River channel are station 13132520 (upstream of the new facility but below the INEEL Diversion) and station 13132535 (downstream at the Lincoln Boulevard Bridge). Water year 1999 and 2000 flow data for these stations are presented in Figure B. Figure C presents flow history of the river as measured at station 13132520 from July 1984 to October 1999. 
Michael Lewis

March 7, 2001

Page 5

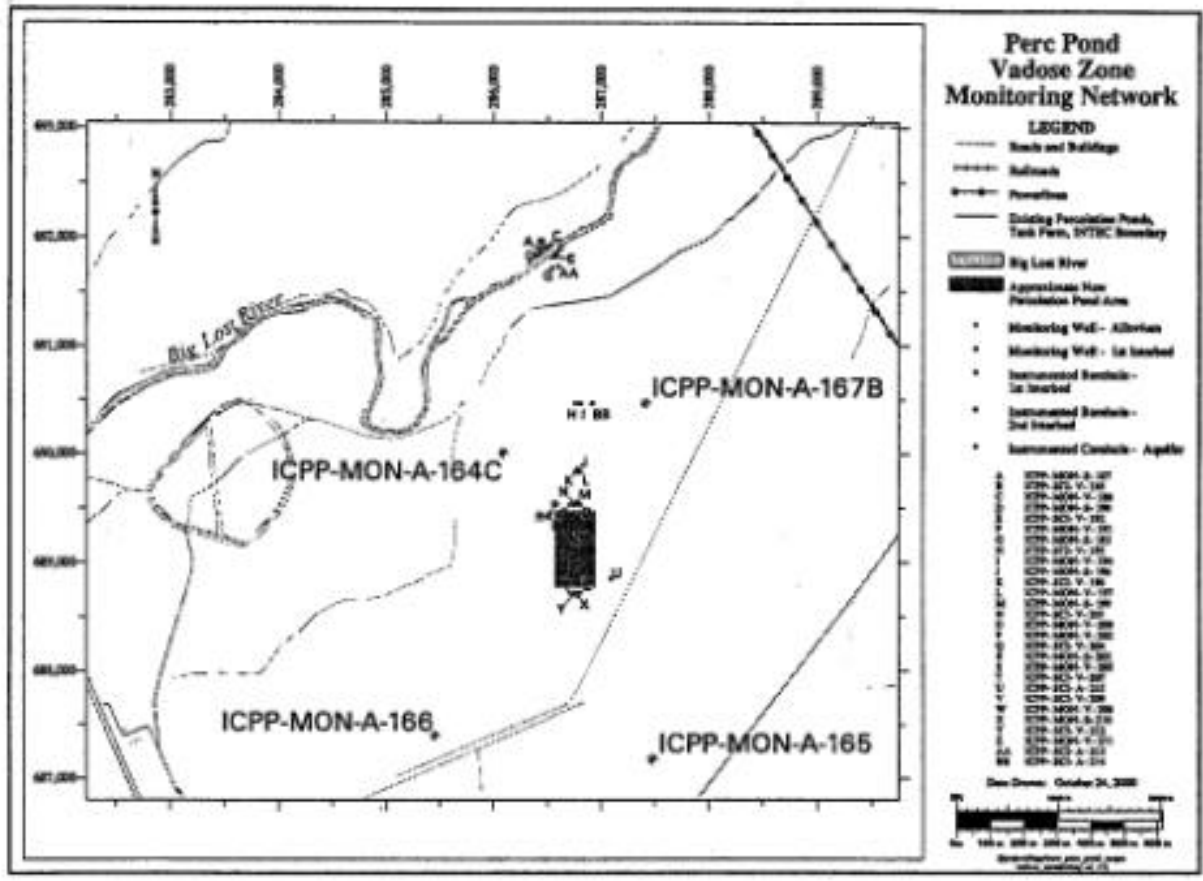

Figure A. Locations of wells around the new INTEC percolation ponds. 
Michael Lewis

March 7, 2001

Page 6

Table A. Wells completed as of February 2001 - INTEC Service Waste Discharge Facility

\begin{tabular}{|c|c|c|c|c|c|c|c|c|c|c|}
\hline \multirow{2}{*}{$\begin{array}{l}\text { Date } \\
\text { Drilled } \\
\text { (mo/yr) }\end{array}$} & \multirow[t]{2}{*}{$\begin{array}{l}\text { Map } \\
\text { Code }\end{array}$} & \multirow{2}{*}{$\begin{array}{c}\text { Well ID } \\
\text { number } \\
\text { (ICPP-XOXX. } \\
\text { X-XXX) }\end{array}$} & \multirow{2}{*}{$\begin{array}{c}\text { Subsurface } \\
\text { Completion } \\
\text { Zone" }\end{array}$} & \multirow[t]{2}{*}{$\begin{array}{l}\text { Well } \\
\text { Type" }\end{array}$} & \multirow{2}{*}{$\begin{array}{l}\text { Drilled } \\
\text { Depth } \\
\text { (i) }\end{array}$} & \multirow{2}{*}{$\begin{array}{l}\text { Completion } \\
\text { Interval } \\
\text { (f) }\end{array}$} & \multirow[t]{2}{*}{$\begin{array}{l}\text { Instrumentation/ } \\
\text { Completion }\end{array}$} & \multicolumn{3}{|c|}{$\begin{array}{l}\text { Water Levels } \\
\text { (ft brop) }\end{array}$} \\
\hline & & & & & & & & $\operatorname{Jan} 2001$ & Feb 2 & 2001 \\
\hline 900 & $\mathrm{~A}$ & MON-S-187 & $A L$ & MW & 49.4 & $43-48.1$ & PVC casing'sereet & & & \\
\hline & B & SCl.V-189 & F1 & IB & \multicolumn{3}{|c|}{ Not completed } & & & \\
\hline $9 / 00$ & $\mathrm{C}$ & MON-V-188 & FI & MW & 120.5 & $114.8-119.8$ & PVC casing/screen & dry & \multicolumn{2}{|c|}{$d r y$} \\
\hline $9 / 00$ & D & MON-S. 190 & AL & MW & 40 & $34.05-392$ & PVC caring/sereen & & & \\
\hline 900 & & MON-S-190A & AL. & MW & & & PVC casing/sereen & & & \\
\hline & $\mathrm{E}$ & SCl-V-192 & FI & IB & \multicolumn{3}{|c|}{ Not completed } & & & \\
\hline 900 & F & MON-V-191 & FI & MW & 119.6 & $113.7-118.7$ & PVC casing/screen & $d y$ & \multicolumn{2}{|c|}{ dry } \\
\hline $8 / 00$ & $\mathrm{G}$ & MON-S-193 & AL. & MW & 43 & $35.2-40.2$ & PVC casing/screen & & & \\
\hline & $\mathrm{H}$ & SCI-V-19S & F1 & 19 & \multicolumn{3}{|c|}{ Not completed } & & & \\
\hline 9,00 & I & MON-V-194 & FI & MW & 163 & $123-128$ & PVC casing/screen & 133 & \multicolumn{2}{|c|}{ dry } \\
\hline 1000 & $\mathrm{~J}$ & MON-S-196 & AL & MW & 60 & $53.4-58.4$ & PVC casinz/screen & & & \\
\hline & $\mathrm{K}$ & SCl-V-198 & F1 & IB & \multicolumn{3}{|c|}{ Not completed } & & & \\
\hline 1000 & $\mathrm{~L}$ & MON-V-197 & FI & $\mathrm{MW}$ & 126.3 & $120.8-125.8$ & PVC casing/screen & $d x y$ & \multicolumn{2}{|c|}{ dry } \\
\hline 1000 & $\mathrm{M}$ & MON-S.199 & AL & MW & 51.5 & $46-51$ & PVC casing/screen & & & \\
\hline & $\mathrm{N}$ & SCI.V.201 & si & GP & \multicolumn{3}{|c|}{ Not completed } & & & \\
\hline & 0 & $\mathrm{MON}-\mathrm{V}-200$ & F1 & $\mathrm{MW}$ & 128.2 & $122-127$ & PVC casing/screen & $d y$ & \multicolumn{2}{|c|}{ dry } \\
\hline & P & $\mathrm{MON}-\mathrm{V}-202$ & F1 & MW & 136 & $1263-131.3$ & PVC casing/screen & 121 & \multicolumn{2}{|c|}{ dry } \\
\hline & & $\begin{array}{l}\text { MON-V. } \\
202 \mathrm{~A}\end{array}$ & FI & MW & 123.7 & $117.5-122.5$ & PVC casing/sereen & & & \\
\hline & Q & SCl-V-204 & FI & IB & \multicolumn{3}{|c|}{ Not completed } & & & \\
\hline & $\mathrm{R}$ & MON-S-203 & $\overline{A L}$ & $\mathrm{MW}$ & 54 & 47.52 & PVC casing/serven & & & \\
\hline & $s$ & MON-V-205 & $F 1$ & 直 & \multicolumn{3}{|c|}{ Net completed } & & & \\
\hline & $\mathrm{T}$ & SCl.V-207 & F & IB & \multicolumn{3}{|c|}{ Not completed } & & & \\
\hline & V & SCI-V-209 & SI & GP & \multicolumn{3}{|c|}{ Not completed } & & & \\
\hline & $\bar{W}$ & MON-V-203 & $F 1$ & MW & \multicolumn{3}{|c|}{ Not completed } & & & \\
\hline 1000 & $\mathrm{X}$ & MON-S-210 & AL & MW & 62 & $54.3-59.3$ & PVC casing/sereen & & & \\
\hline & $Y$ & $5 \mathrm{Cl}-\mathrm{V}-212$ & SI & GP & \multicolumn{3}{|c|}{ Not completed } & & & \\
\hline 1000 & $\mathrm{z}$ & MON-V-211 & F1 & MW & 130.5 & $123.9-128.9$ & PVC casing/screen & dry & dr & $y$ \\
\hline & $\begin{array}{c}\text { Core } \\
1\end{array}$ & SCI-A-215 & DI & IB & & Not coms & eted & & & \\
\hline & $\begin{array}{c}\text { Core } \\
2\end{array}$ & SCI-V-214 & DI & IB & & Not com & sted & & & \\
\hline $9 / 00$ & $\begin{array}{c}\text { Core } \\
3\end{array}$ & SCI-V-213 & $\overline{\mathrm{DI}}$ & GP & 560 & na & PVC casing/sereen & & & \\
\hline $\begin{array}{c}6 / 00 \\
v L 6 / 00\end{array}$ & 1 & $\begin{array}{l}\text { MON-A- } \\
164 \mathrm{C}\end{array}$ & AQ & MW & 550 & $487-527$ & SS cating/screen & $\begin{array}{c}493.2 \\
11 / 1400\end{array}$ & $\begin{array}{l}498.3 \\
1 / 901\end{array}$ & $\begin{array}{l}481.2 \\
26601\end{array}$ \\
\hline $\begin{array}{c}4 / 00 \\
\text { VLS/00 }\end{array}$ & 2 & MON-A-165 & AQ & MW & 555 & $485-525$ & SS casing/screen & $\begin{array}{c}495.8 \\
11 / 1400 \\
\end{array}$ & $\begin{array}{l}469.9 \\
1 / 9101 \\
\end{array}$ & $\begin{array}{l}496.2 \\
27701 \\
\end{array}$ \\
\hline $\begin{array}{l}3-4 / 00 \\
V \mathcal{L} / 00\end{array}$ & 3 & MON-A-166 & $\overline{A Q}$ & MW & 547 & $487-527$ & SS casing/screen & $\begin{array}{c}495 \\
10 / 3000\end{array}$ & ns & ns \\
\hline $\begin{array}{c}5 / 00 \\
\text { vL } 6 / 00\end{array}$ & 4 & $\begin{array}{c}\text { MON-A- } \\
167 \mathrm{~B}\end{array}$ & $\overline{A Q}$ & MW & 543 & $462-502$ & SS casing/screen & $\begin{array}{c}4928 \\
11 / 2900\end{array}$ & $\begin{array}{l}492.9 \\
1 / 9.01 \\
\end{array}$ & $\begin{array}{l}4928 \\
21601\end{array}$ \\
\hline
\end{tabular}

a. $\mathbf{A L}=$ alluvium

FI = first interbed

SI - socond interbed

$\mathrm{AQ}=$ aquifer

$\mathrm{DI}=$ decp interbed b. $M W=$ monitoring well

IB - instrumented borchole

GP - geophysical borehole ns - not sampled

na - not applicable

$\mathrm{VL}$ - video log taken 
Michael Lewis

March 7, 2001

Page 7

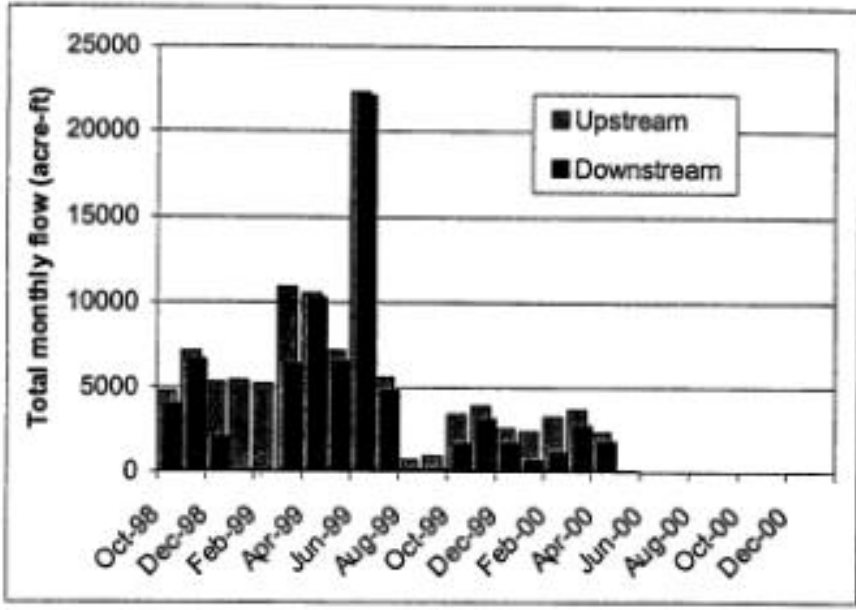

Figure B. Monthly discharge in the Big Lost River upstream and downstream of the site of the new INTEC percolation ponds for water years 1999 and 2000.

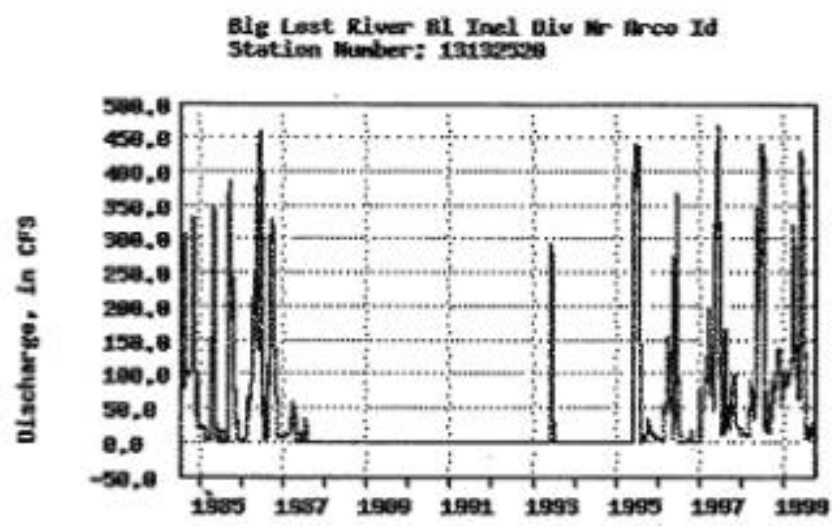

Legend: - discharge, in crs

Estimated Discharge, in CFS

Figure C. Daily average discharge in the Big Lost River upstream of the new INTEC percolatin ponds from July 1984 to October 1999. 
Michael Lewis

March 7, 2001

Page 8

Measurements of perched water in completed wells occurred in January and February 2001. In wells completed in the first interbed located approximately $150-170 \mathrm{ft}$ bls, all but two wells were dry during two measurement events. Water was detected in ICPP-MON-V-194 in January 2001. Approximately $10 \mathrm{ft}$ of standing water was measured in ICPP-MON-V-202 during the same month. However, during the second measurement event in February 2001, the standing water in both wells had dissipated and the wells were dry. Geologist logs of these holes only note cuttings as being slightly moist during drilling and video logs of the open holes were not available. These detections of water, although suggesting the presence of perched water, could be attributed to the Big Lost River or to water injected into the boreholes during drilling. Two coreholes in the vicinity of the new ponds were drilled by the USGS (USGS-185 and USGS-186, not shown on Figure A) in the fall of 2000. The video log for USGS-186 (the closer of the two to the Big Lost River channel) showed water entering the hole at the 299-ft level in October 2000 and in February 2001. This implies that perched water is still present beneath the new ponds at a depth of approximately $300 \mathrm{ft}$ bls.

Knowledge of the lateral extent of perched water originating from both the BLR and the percolation ponds may provide some information regarding the interaction and relative impacts each perched water body may have on the other. Such impacts may include dilution of the contaminants in the percolation pond-derived perched water and a greater areal boundary of the combined perched water bodies. There have been no measured or modeled predictions of perched water extent from Big Lost River infiltration during channel flow. However, perched water was observed in video logs of boreholes ICPP-MON-A-164 (A, B, and C) and ICPP-MON-A-167 at depths of 170,198 , and $235 \mathrm{ft}$ bls. At their shortest distance, these wells are located approximately 800 and $1700 \mathrm{ft}$, respectively, from the Big Lost River channel. Perching water at a depth of $299 \mathrm{ft}$ bls was also observed in the video logs of corehole USGS-186, located approximately $3200 \mathrm{ft}$ from the channel. A radius of 1900-ft from the center of the currently-used ponds (south of the INTEC facility) approximately encompasses the measured lateral extent of perched water. WAG-3 OU3-13 modeling predicted a lateral spread radius of $4000 \mathrm{ft}$ from the center of the ponds. However, a more realistic estimate of the lateral spread radius is approximately $1500-1900 \mathrm{ft}$ from the center of the ponds (DOE/ID 1999).

Preliminary stratigraphic cross-sections through the new facility are presented in Attachment B. Because few of the boreholes were geophysically logged after drilling, cross-sections were constructed using lithologic descriptions from geologist logs. More detailed information, as obtained, will be incorporated in the future. The thickness of surficial alluvium at the new facility ranges from $40 \mathrm{ft}$ to $60 \mathrm{ft}$. Interbeds occurring between $80-90 \mathrm{ft}, 117-152 \mathrm{ft}, 180-236 \mathrm{ft}$, and 320 $330 \mathrm{ft}$ bls may be continuous across the area covered by the well field. Additional interbeds observed in some boreholes between $275-308 \mathrm{ft}$ and $425-475 \mathrm{ft}$ bls may be discontinuous across the area. Two deep coreholes, USGS-185 and USGS-186, were drilled by the US Geological Survey near the new ponds (not shown on the map). Although both holes have been logged for geophysical properties, none of the core has been logged for lithologic identification. Interbed materials collected during all drilling operations have not been analyzed for hydraulic or physical properties. 
Michael Lewis

March 7, 2001

Page 9

The following well-specific summaries are notes compiled from geologist and field team leader logbooks relative to water detected in the boreholes during drilling and well construction. All wells drilled after ICPP-MON-A-164A were drilled with water injected into the borehole. In most cases, the notes are cryptic and provide limited information on the conditions of the borehole.

ICPP-MON-A-164A (drilled 1-2/00?)

$45-46 \mathrm{ft}$ bls some water above bedrock

Notes recorded on 1-5-00 indicate water at $40 \mathrm{ft}$

$158 \mathrm{ft}$ bls wet clay

$177 \mathrm{ft}$ bls very wet rubble zone

ICPP-MON-A-164B (drilled 2/00?)

$122 \mathrm{ft}$ bls water on top of basalt, in rubble above interbed

$125-140 \mathrm{ft}$ bls interbed

$139 \mathrm{ft}$ bls damp red clay

Video log taken $2 \& 3 / 00$

Standing water in the hole at $117 \mathrm{ft}$ bls. Hole cased and drilling continued. Water observed dripping down the inside of the casing beginning at $130 \mathrm{ft}$. At $170 \mathrm{ft}$ bls, voluminous amounts of water cascading down the borehole. Further visual observation obscured.

ICPP-MON-A-164C (drilled 6/00)

No mention of water or wetness in the borehole during drilling

Video log taken $6 / 00$

Wet borehole walls from $110 \mathrm{ft}$ to $235 \mathrm{ft}$ bls. Cascading water from the 235 - $\mathrm{ft}$ zone down to total depth.

ICPP-MON-A-165 (drilled 4/00)

No mention of water or wetness in the borehole during drilling

Video taken 5/00

Wet borehole walls were observed at $231,271,375,391$, and $412 \mathrm{ft}$ bls. No cascading water.

ICPP-MON-A-166 (drilled 3-4/00)

$440 \mathrm{ft}$ bls dampness

Video log taken $4 / 00$

No visible cascading water. At $295 \mathrm{ft}$ bls, borehole walls looked more wet and at $437 \mathrm{ft}$ bls they were very wet. The borehole was not cleaned out after drilling; consequently, mud cake on the borebole walls prevented further observation.

ICPP-MON-A-167 (drilled 5/00)

No mention of water or wetness in the borehole during drilling

Video log taken $6 / 00$

Water cascading from borehole walls at $198 \mathrm{ft}$ bls, approximate location of bottom of stabilization casing down to total depth. Oxidized basalt at 260,306 , and $320 \mathrm{ft}$ bls may indicate water sources however, it was impossible to distinguish from water originating from above. 
Michael Lewis

March 7, 2001

Page 10

ICPP-MON-S-187 (drilled 9/00)

No mention of water or wetness in the borehole during drilling

ICPP-MON-S-190 (drilled 9/00)

No mention of water or wetness in the borehole during drilling

ICPP-MON-S-190A (drilled 9/00)

No mention of water or wetness in the borehole during drilling

ICPP-MON-S-193 (drilled 8/00)

$41 \mathrm{ft}$ bls basalt

ICPP-MON-S-196 (drilled 10/00)

$38 \mathrm{ft}$ bls moist/wet

$40 \mathrm{ft}$ bls moist

$45 \mathrm{ft}$ bls moist

$51 \mathrm{ft}$ bls dry

$53 \mathrm{ft}$ bls dry

ICPP-MON-S-199 (drilled 10/00)

$10 \mathrm{ft}$ bls slightly moist

$15 \mathrm{ft}$ bls slightly moist

$20 \mathrm{ft}$ bls dry

$25 \mathrm{ft}$ bls dry

$30 \mathrm{ft}$ bls slightly moist

$40 \mathrm{ft}$ bls dry

$45 \mathrm{ft}$ bls dry

$50 \mathrm{ft}$ bls dry

ICPP-MON-S-210 (drilled 10/00)

Entire hole moist to slightly moist

ICPP-MON-V-188 (drilled 9/00)

57-72 ft bls moist sediments/clay

$88-90 \mathrm{ft}$ bls moist clay

ICPP-MON-V-191 (drilled 9/00)

$60 \mathrm{ft}$ bls moist sediments

$115 \mathrm{ft}$ bls moist

$117 \mathrm{ft}$ bls moist clay

ICPP-MON-V-194 (drilled 9/00)

$40 \mathrm{ft}$ bls slightly moist

$52-53 \mathrm{ft}$ bls slightly moist 
Michael Lewis

March 7, 2001

Page 11

$97 \mathrm{ft}$ bls slightly moist

$100 \mathrm{ft}$ bls slightly moist

$125 \mathrm{ft}$ bls slightly moist

ICPP-MON-V-197 (drilled 10/00)

$15 \mathrm{ft}$ bls moist

20-56 ft bls moist

56-106 ft bls dry

$106-118.5 \mathrm{ft}$ bls moist

$120 \mathrm{ft}$ bls wet

$125 \mathrm{ft}$ bls moist

ICPP-MON-V-211 (drilled 10/00)

$5 \mathrm{ft}$ bls slightly moist

$10 \mathrm{ft}$ bls slightly moist

$25 \mathrm{ft}$ bls slightly moist

45-47 ft bls near top of interbed, moist

$110 \mathrm{ft}$ bls very moist

$125 \mathrm{ft}$ bls slightly moist

$127 \mathrm{ft}$ bls slightly moist

$129 \mathrm{ft}$ bls moist

ICPP-SCI-A-213 (drilled 9/00)

$58 \mathrm{ft}$ bls moist interbed (gamma log shows interbed at $57-66 \mathrm{ft}$ bls)

No other mention of water or wetness in the borehole during drilling

ICPP-SCI-V-195 (drilled 1/01)

Drilling of well 195, attempt at core recovery (January 31, 2001) was unsuccessful because the sediment in the 140-150 $\mathrm{ft}$ interbed was too dry to be held in place within the core barrel during extraction of the drill string.

USGS-185 (drilled 9/00, not shown on the map but located approximately $310 \mathrm{ft}$ northeast of ICPP-MON-V-211)

Video logs taken September 2000 and February 2001

$9 / 00=108 \mathrm{ft}$ bis, borehole walls very wet

$157 \mathrm{ft}$ bls, water seeping in through casing joint (after cased)

$220 \mathrm{ft}$ bls, wet borehole walls

274-289 ft bls, very wet borehole walls

$310 \mathrm{ft}$ bls, wet borehole walls

$321 \mathrm{ft}$ bls, very wet walls

$2 / 01$ - $214-224 \mathrm{ft}$ bls, wet and dripping down borehole walls

280-307 ft bls, wet borehole walls 
Michael Lewis

March 7, 2001

Page 12

USGS-186 (drilled 10/00, not shown on map but located approximately $100 \mathrm{ft}$ east of ICPPMON-V-194)

Videos log taken October 2000 and February 2001

$10 / 00-190-299 \mathrm{ft}$ bls, very wet borehole walls

$299 \mathrm{ft}$ bls, water squirting into borehole from 1 spot

$299 \mathrm{ft}$ to water table, walls very wet

$2 / 01$ - $204 \mathrm{ft}$ bls, walls moist

218-242 ft bls, moist with rivulets of water along borehole wall

$248 \mathrm{ft}$ bls, wet fractures but dry walls

$298 \mathrm{ft}$ bls, wet walls

$299 \mathrm{ft}$ bls, water still squirting into borehole from 1 spot

$299-489 \mathrm{ft}$ bls, borehole walls moist to wet with some dripping 
Michael Lewis

March 7, 2001

Page 13

\section{References}

40 CFR 141-143, "National Primary and Secondary Drinking Water Regulations," Code of Federal Regulations, Office of the Federal Register, current issue.

DOE Order 5400.5, June 1990. "Radiation Protection of the Public and the Environment," U.S. Department of Energy, Washington, D.C.

DOE-ID, 1997, Comprehensive RI/FS for the Idaho Chemical Processing Plant WAG 3 OU 3-13 at the INEEL-Part A RI/BRA Repont (Final), U.S. Department of Energy, Idaho Operations Office, DOE/ID-10534, November.

DOE-ID, 1999a, Final Record of Decision, Idaho Nuclear Technology and Engineering Center, DOE/ID10660, U.S. Department of Energy Idaho Operations Office, October.

DOE-ID, 1999c, Idaho High-Level Waste and Facilities Disposition, Draft Environmental Impact Statement, DOE/EIS-02870, U.S. Department of Energy Idaho Operations Office, December.

DOE-ID, 1999, Evaluation and Site Selection for a New Service Waste Disposal Facility for the Idaho Nuclear Technology and Engineering Center, DOE/ID-10705, Rev. 0, Department of Energy Idaho Operations Office, September.

Forsythe, H., S. Losinski, and D. Taylor, 1998, INTEC Percolation Pond Engineering Study, Engineering Design File EDF-724, Revision 0, July.

Graham, J., INEEL, to G. Eager, IDEQ, January 3, 2000, “Wastewater Land Application Permit Application for the Idaho Nuclear Engineering Center New Percolation Ponds."

IDAPA 16.01.17, April 1988, "Wastewater Land Application Permit Rules," Office of Administrative Rules, State of Idaho.

IDAPA 58.01.11, March 1997, "Ground Water Quality Rule," Office of Administrative Rules, State of Idaho.

IDHW, 1996, Handbook for Land Application of Municipal and Industrial Wastewater, Department of Health and Welfare, State of Idaho, April.

Johnston, J., Regional Administrator, IDEQ, to D. Walker, INEEL, June 5, 2000. 
Michael Lewis

March 7, 2001

Page 14

pgs

Attachments

ce: S. L. Ansley Letter File

L. C. Hull Letter File

Uniform File Code: 1015

Disposition Authority: Destroy WNLN

Retention Schedule: Unscheduled

NOTE: Original disposition authority, retention schedule, and Uniform Filing Code applied by the sender may not be appropriate for all recipients. Make adjustments as needed. 


\section{Attachments}




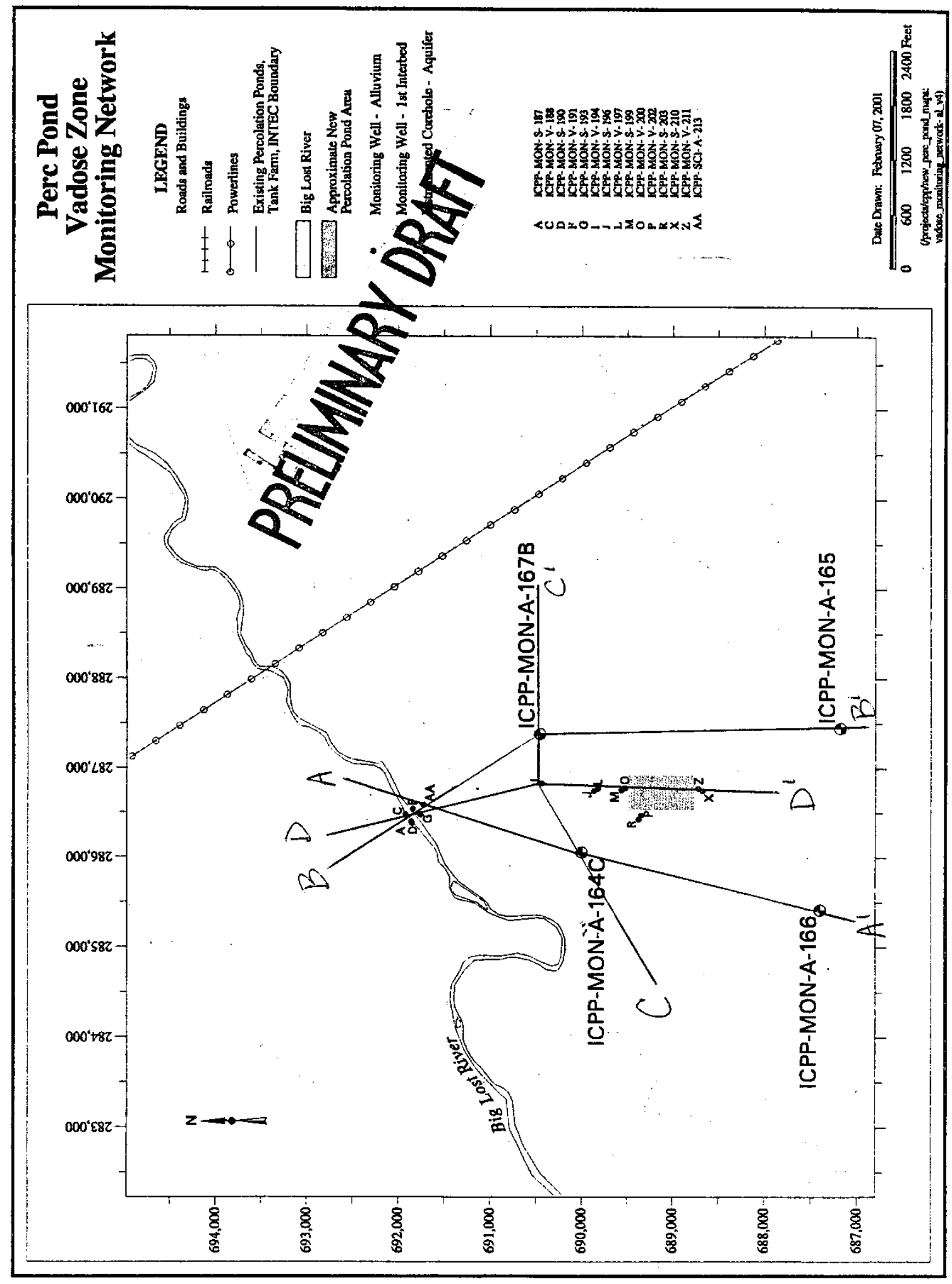




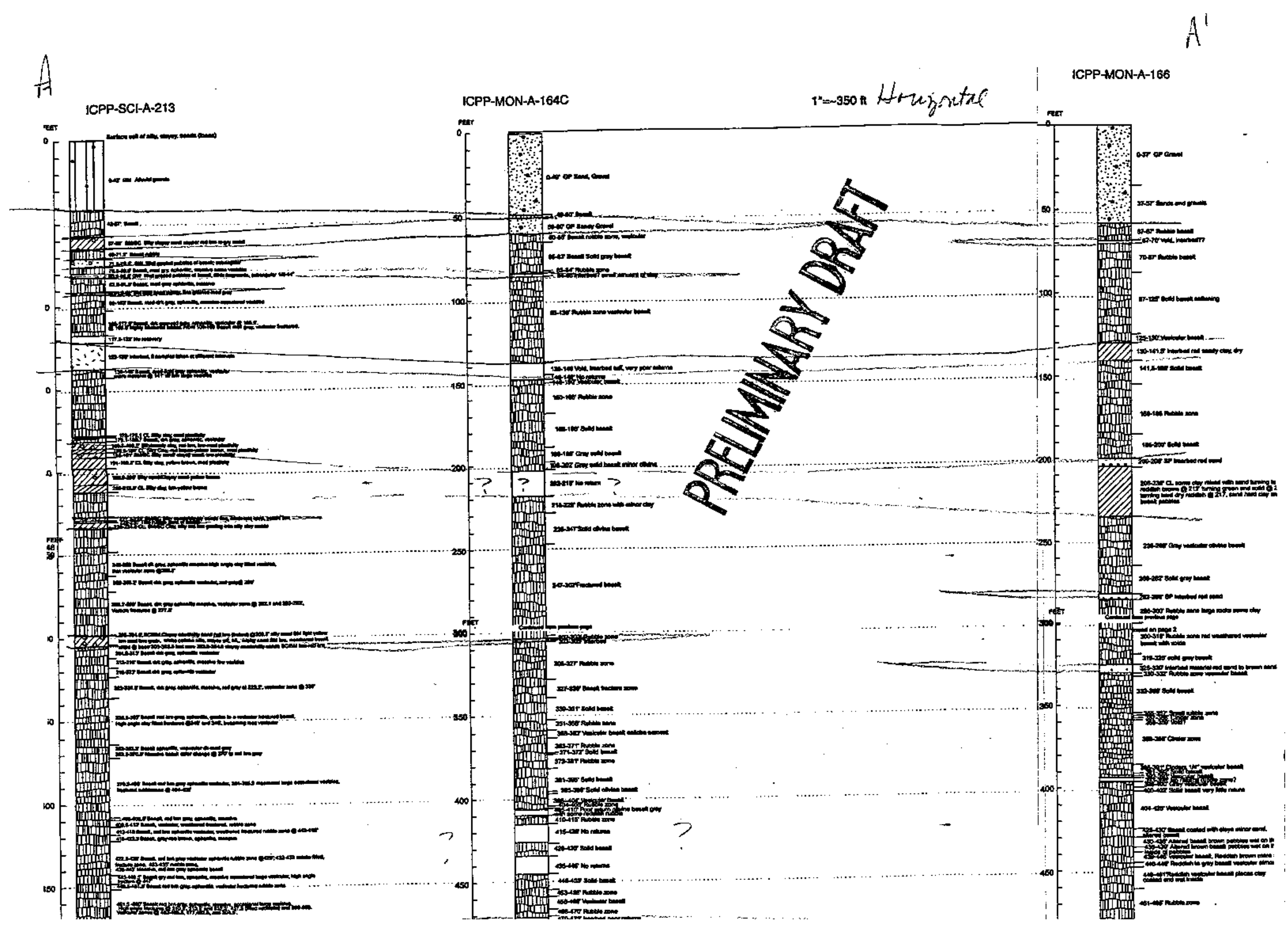




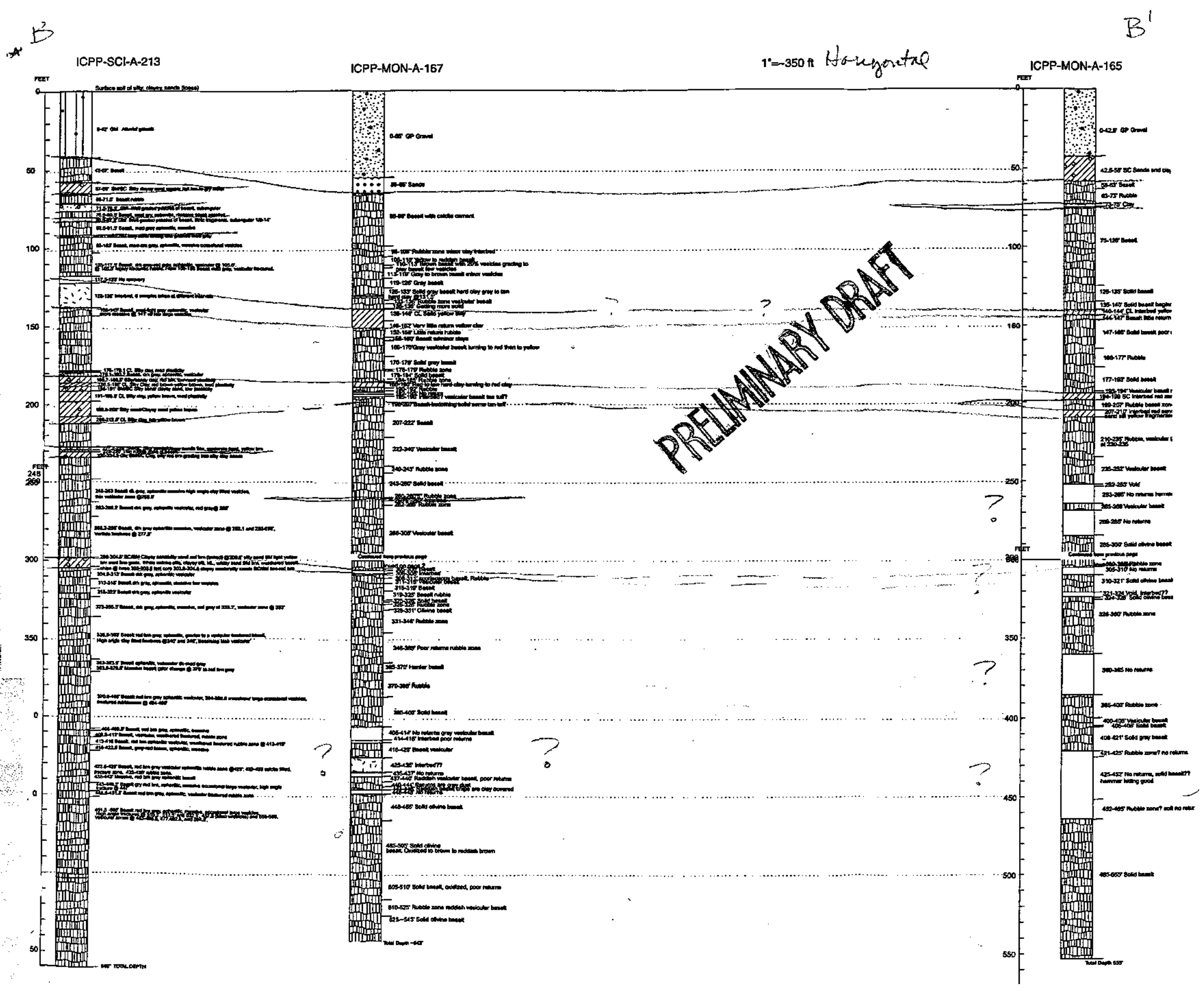




$$
1+1
$$


$D$

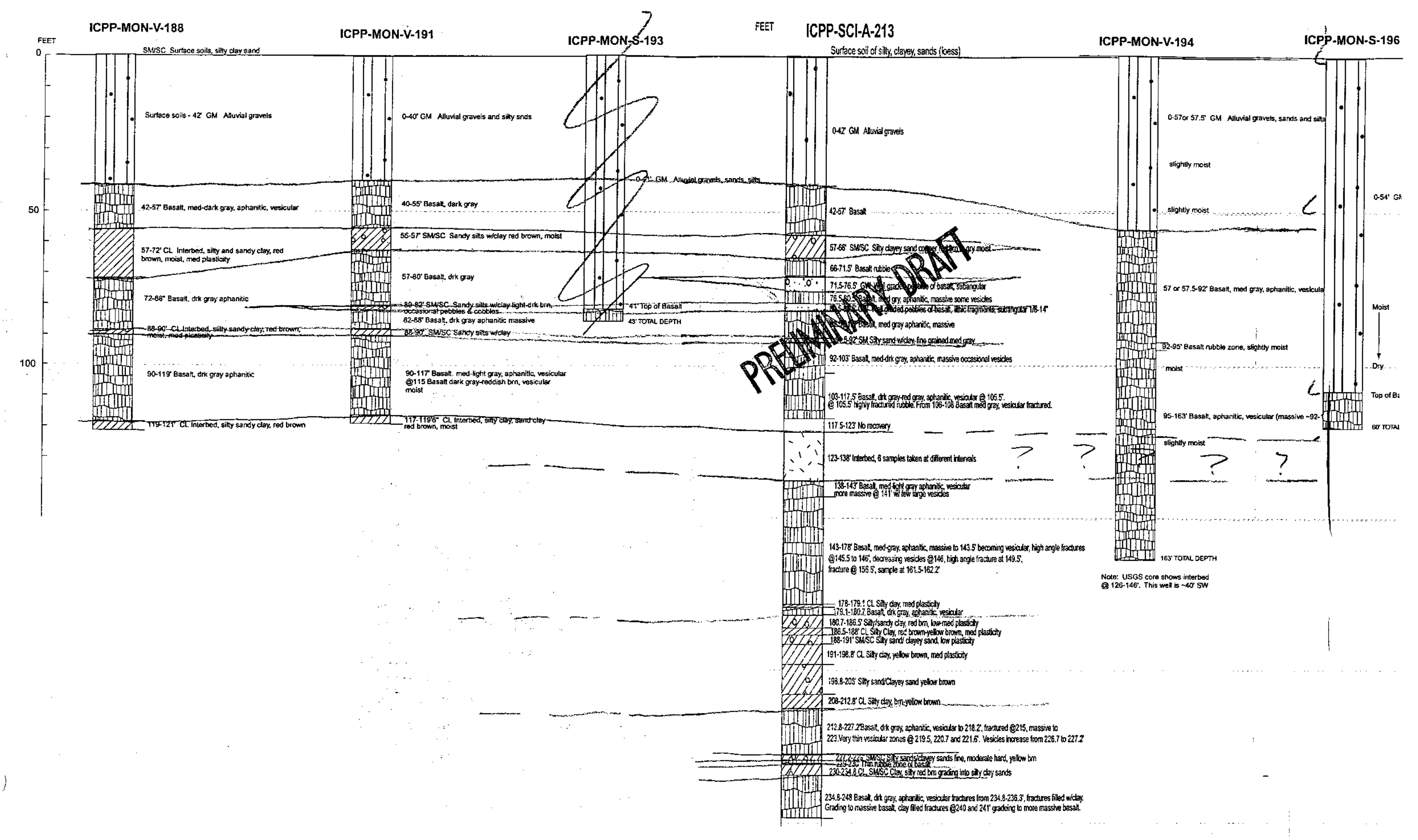




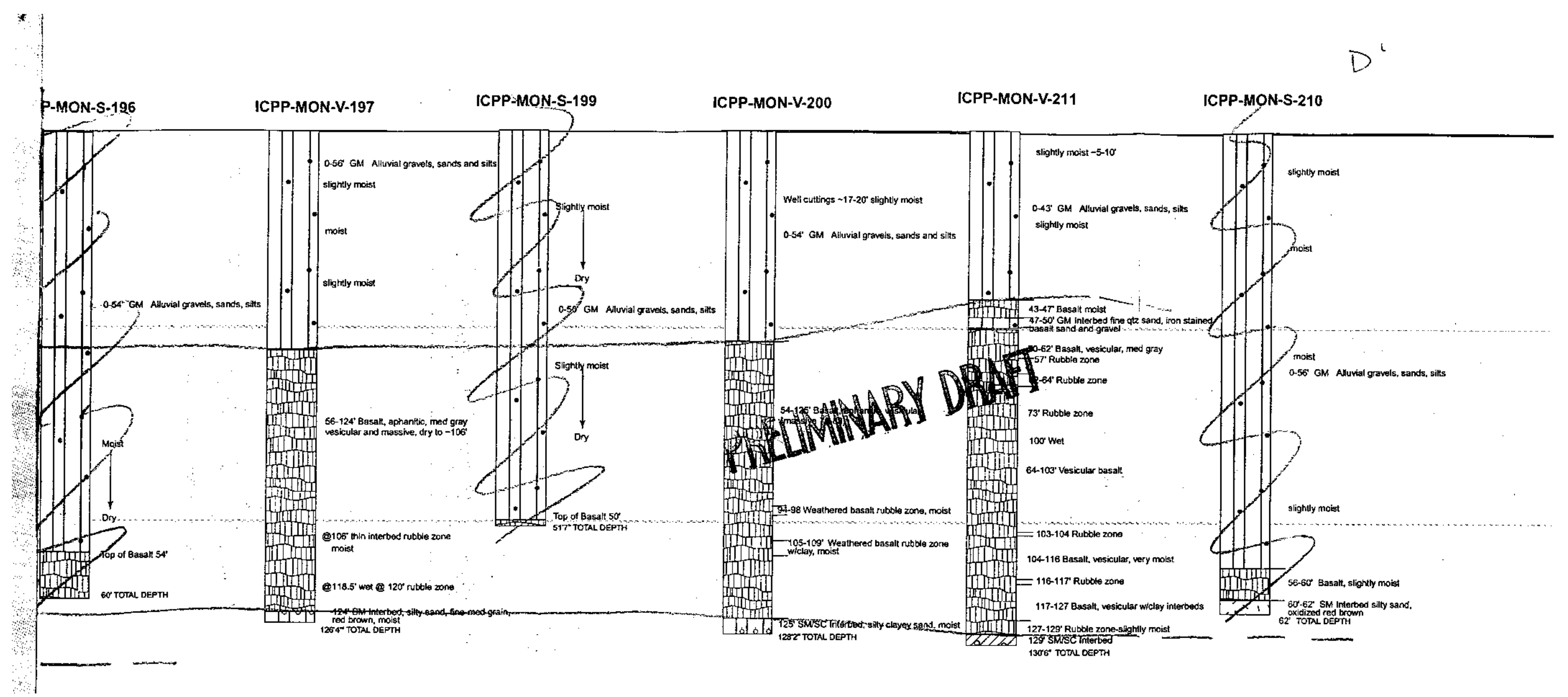




\section{Appendix C \\ Preoperational Groundwater Data}




$$
\text { C-2 }
$$




\section{NOTES}

\section{General:}

NS - not sampled

TAL - target analyte list

CLP - Contract Laboratory Program

LQF - Laboratory-Assigned Concentration Qualifier Flags

VQF - Validator-Assigned Data Qualifier Flags

$\mathrm{COL} / \mathrm{DL}$ - coliform per deciliter

\section{Inorganic Data:}

LQF: $\quad$ B - Value was obtained from a reading that was less than the Contract Required Detection Limit but greater than or equal to the Instrument Detection Limit.

$\mathrm{U}$ - Analyte was analyzed for but not detected.

E - The reported value is estimated because of the presence of interference.

VQF: $\quad \mathrm{U}$ - The material was analyzed for and was detected at or above the applicable detection limit, but the associated value was less than 5 times the highest positive amount in any laboratory blank.

$\mathrm{UJ}$ - The material was analyzed for, but was not detected.

$\mathbf{J}$ - The material was analyzed for and was detected at or above the applicable detection limit but the associated value is an estimate and may be inaccurate or imprecise.

$\mathrm{R}$ - The accuracy of the data is so questionable that it is recommended the data not be used.

\section{Organic Data:}

LQF: U - Compound was analyzed for but not detected.

$\mathrm{J}$ - Estimated value.

B - Analyte is found in the associated blank as well as the sample.

E - Concentration of compound exceeded the calibration range of the GC or GC/MS instrument for that specific analysis.

VQF: U - Material was analyzed for but not detected. The associated numerical value is the sample quantitation limit.

$\mathrm{J}$ - Associated numerical value is an estimated quantity

$\mathrm{R}$ - The data are unusable.

UJ - The material was analyzed for, but was not detected. The sample quantitation limit is an estimated quantity. 


\section{Radiochemistry Data:}

VQF: U - The analysis was performed, but no radioactivity was detected (i.e., the radioanalytical result was not statistically positive at the $95 \%$ confidence level) meaning the radionuclide is not considered to be present in the sample.

UJ - The analysis was performed and the result is highly questionable due to serious analytical error and/or laboratory quality control anomalies. Use of data is strongly discouraged.

$\mathrm{J}$ - The analysis was performed and radioactivity was detected (i.e., the radioanalytical result was statistically positive at the $95 \%$ confidence level), however, the result is questionable due to analytical and/or laboratory quality control anomalies and should therefore be used only as an estimated quantity.

$\mathrm{R}$ - The analysis result is unusable and was rejected due to severe analytical and/or quality control problems. 
Table C-1. Baseline groundwater data for Well ICPP-MON-A-164C.

\begin{tabular}{|c|c|c|c|c|}
\hline \multirow[b]{2}{*}{ Analyzed Constituents } & \multicolumn{4}{|c|}{ Sample Dates } \\
\hline & $\begin{array}{c}\text { November } \\
2000\end{array}$ & $\begin{array}{c}\text { January } \\
2001\end{array}$ & $\begin{array}{c}\text { February } \\
2001\end{array}$ & $\begin{array}{c}\text { March } \\
2001\end{array}$ \\
\hline Miscellaneous Inorganics & $(\mathrm{mg} / \mathrm{L})$ & $(\mathrm{mg} / \mathrm{L})$ & $(\mathrm{mg} / \mathrm{L})$ & $(\mathrm{mg} / \mathrm{L})$ \\
\hline Alkalinity & 149 & 148 & 144 & 144 \\
\hline Biochemical Oxygen Demand & $\mathrm{U}$ & $9.14 \mathrm{~B}$ & $3.60 \mathrm{~B}$ & NS \\
\hline Bromide & NS & NS & $\mathrm{U}$ & NS \\
\hline Chloride & NS & NS & 5.8 & NS \\
\hline Chromium hexavalent & NS & $\mathrm{R}$ & $\mathrm{U}$ & NS \\
\hline Fecal Coliform (COL/DL) & None & None & None & NS \\
\hline Total Coliform (COL/DL) & None & None & 950 & NS \\
\hline Fluoride & NS & NS & 0.22 & NS \\
\hline Nitrate (mg N/L) & 0.4 & 0.45 & 0.46 & NS \\
\hline Nitrite (mg N/L) & $\mathrm{U}$ & $\mathrm{U}$ & $\mathrm{U}$ & NS \\
\hline Nitrogen, Ammonia (as N) & $\mathrm{U}$ & $\mathrm{U}$ & $\mathrm{U}$ & $\mathrm{U}$ \\
\hline Nitrogen, Nitrate-Nitrite (as N) & 0.484 & 0.486 & 0.496 & 0.482 \\
\hline Phosphate & NS & NS & $\mathrm{U}$ & NS \\
\hline Phosphorous, Total (as P) & 0.0486 & 0.0361 & 0.0414 & 0.0262 \\
\hline Sulfate & NS & NS & 22.2 & NS \\
\hline Total Dissolved Solids & 210 & 217 & 214 & 151 \\
\hline Total Kjeldahl Nitrogen & $\mathrm{U}$ & 0.132 & $\mathrm{U}$ & $\mathrm{U}$ \\
\hline TAL Metals & $(\mathrm{mg} / \mathrm{L})$ & $(\mathrm{mg} / \mathrm{L})$ & $(\mathrm{mg} / \mathrm{L})$ & $(\mathrm{mg} / \mathrm{L})$ \\
\hline Aluminum & $\mathrm{U}$ & $\mathrm{U}$ & $\mathrm{U}$ & $\mathrm{U}$ \\
\hline Antimony & $\mathrm{U}$ & $\mathrm{U}$ & $\mathrm{U}$ & $\mathrm{U}$ \\
\hline Arsenic & 0.006 & $\mathrm{U}$ & $\mathrm{U}$ & $\mathrm{U}$ \\
\hline Barium & 0.0764 & 0.0697 & 0.0671 & 0.071 \\
\hline Beryllium & $\mathrm{U}$ & $\mathrm{U}$ & $\mathrm{U}$ & $\mathrm{U}$ \\
\hline Boron & 0.0348 & $\mathrm{U}$ & $\mathrm{U}$ & $\mathrm{U}$ \\
\hline Cadmium & $\mathrm{U}$ & $\mathrm{U}$ & $\mathrm{U}$ & $\mathrm{U}$ \\
\hline Calcium & 47.3 & 45.3 & 43.1 & 45.2 \\
\hline Chromium & 0.006 & $\mathrm{U}$ & $\mathrm{U}$ & $\mathrm{U}$ \\
\hline Cobalt & $\mathrm{U}$ & $\mathrm{U}$ & $\mathrm{U}$ & $\mathrm{U}$ \\
\hline Copper & 0.0053 & 0.0063 & $\mathrm{U}$ & $\mathrm{U}$ \\
\hline Iron & 0.117 & 0.130 & $\mathrm{U}$ & $\mathrm{U}$ \\
\hline Lead & $\mathrm{U}$ & $\mathrm{U}$ & $\mathrm{U}$ & $\mathrm{U}$ \\
\hline Lithium & $\mathrm{U}$ & $\mathrm{U}$ & $\mathrm{U}$ & $\mathrm{U}$ \\
\hline Magnesium & 12.1 & 11.4 & 10.8 & 11.6 \\
\hline
\end{tabular}


Table C-1. (continued).

\begin{tabular}{|c|c|c|c|c|}
\hline \multirow[b]{2}{*}{ Analyzed Constituents } & \multicolumn{4}{|c|}{ Sample Dates } \\
\hline & $\begin{array}{c}\text { November } \\
2000 \\
\end{array}$ & $\begin{array}{c}\text { January } \\
2001 \\
\end{array}$ & $\begin{array}{c}\text { February } \\
2001 \\
\end{array}$ & $\begin{array}{c}\text { March } \\
2001 \\
\end{array}$ \\
\hline TAL Metals & $(\mathrm{mg} / \mathrm{L})$ & $(\mathrm{mg} / \mathrm{L})$ & $(\mathrm{mg} / \mathrm{L})$ & $(\mathrm{mg} / \mathrm{L})$ \\
\hline Manganese & 0.005 & 0.0181 & $\mathrm{U}$ & $0.0092 \mathrm{~B}$ \\
\hline Mercury & $\mathrm{U}$ & $\mathrm{U}$ & $\mathrm{U}$ & $\mathrm{U}$ \\
\hline Nickel & $\mathrm{U}$ & 0.0112 & $\mathrm{U}$ & $0.0069 \mathrm{~B}$ \\
\hline Potassium & $2.09 \mathrm{~B}$ & $2.03 \mathrm{~B}$ & $1.69 \mathrm{~B}$ & $1.94 \mathrm{~B}$ \\
\hline Selenium & $\mathrm{U}$ & $\mathrm{U}$ & $\mathrm{U}$ & $\mathrm{U}$ \\
\hline Silicon & 10.1 & 9.79 & 9.8 & 10.1 \\
\hline Silver & $\mathrm{U}$ & $\mathrm{U}$ & $\mathrm{U}$ & $\mathrm{U}$ \\
\hline Sodium & 6.84 & 6.58 & 6.24 & 6.37 \\
\hline Strontium & 0.273 & 0.251 & 0.245 & 0.258 \\
\hline Thallium & $\mathrm{U}$ & $\mathrm{U}$ & $\mathrm{U}$ & $\mathrm{U}$ \\
\hline Vanadium & $\mathrm{U}$ & $\mathrm{U}$ & $\mathrm{U}$ & $\mathrm{U}$ \\
\hline Zinc & 0.0113 & 0.0136 & $\mathrm{U}$ & $0.0051 \mathrm{~B}$ \\
\hline CLP-List Volatile Organic Compounds & $(\mathrm{mg} / \mathrm{L})$ & $(\mathrm{mg} / \mathrm{L})$ & $(\mathrm{mg} / \mathrm{L})$ & $(\mathrm{mg} / \mathrm{L})$ \\
\hline 1,1,1-Trichloroethane & $\mathrm{U}$ & $\mathrm{U}$ & $\mathrm{U}$ & $\mathrm{U}$ \\
\hline 1,1,2,2-Tetrachloroethane & $\mathrm{U}$ & $\mathrm{U}$ & $\mathrm{U}$ & $\mathrm{U}$ \\
\hline 1,1,2-Trichloroethane & $\mathrm{U}$ & $\mathrm{U}$ & $\mathrm{U}$ & $\mathrm{U}$ \\
\hline 1,1-Dichloroethane & $\mathrm{U}$ & $\mathrm{U}$ & $\mathrm{U}$ & $\mathrm{U}$ \\
\hline 1,1-Dichloroethene & $\mathrm{U}$ & $\mathrm{U}$ & $\mathrm{U}$ & $\mathrm{U}$ \\
\hline 1,2-Dichloroethane & $\mathrm{U}$ & $\mathrm{U}$ & $\mathrm{U}$ & $\mathrm{U}$ \\
\hline 1,2-Dichloroethene (Total) & $\mathrm{U}$ & $\mathrm{U}$ & $\mathrm{U}$ & $\mathrm{U}$ \\
\hline 1,2-Dichloropropane & $\mathrm{U}$ & $\mathrm{U}$ & $\mathrm{U}$ & $\mathrm{U}$ \\
\hline 2-Butanone & $\mathrm{R}$ & $\mathrm{R}$ & $\mathrm{U}$ & $\mathrm{U}$ \\
\hline 2-Hexanone & $\mathrm{U}$ & $\mathrm{U}$ & $\mathrm{U}$ & $\mathrm{U}$ \\
\hline 4-Methyl-2-pentanone & $\mathrm{U}$ & $\mathrm{U}$ & $\mathrm{U}$ & $\mathrm{U}$ \\
\hline Acetone & $\mathrm{U}$ & $\mathrm{U}$ & $\mathrm{U}$ & $\mathrm{U}$ \\
\hline Benzene & $\mathrm{U}$ & $\mathrm{U}$ & $\mathrm{U}$ & $\mathrm{U}$ \\
\hline Bromodichloromethane & $\mathrm{U}$ & $\mathrm{U}$ & $\mathrm{U}$ & $\mathrm{U}$ \\
\hline Bromoform & $\mathrm{U}$ & $\mathrm{U}$ & $\mathrm{U}$ & $\mathrm{U}$ \\
\hline Bromomethane & $\mathrm{U}$ & $\mathrm{U}$ & $\mathrm{U}$ & $\mathrm{U}$ \\
\hline Carbon disulfide & $\mathrm{U}$ & $\mathrm{U}$ & $\mathrm{U}$ & $\mathrm{U}$ \\
\hline Carbon tetrachloride & $\mathrm{U}$ & $\mathrm{U}$ & $\mathrm{U}$ & $\mathrm{U}$ \\
\hline Chlorobenzene & $\mathrm{U}$ & $\mathrm{U}$ & $\mathrm{U}$ & $\mathrm{U}$ \\
\hline Chloroethane & $\mathrm{U}$ & $\mathrm{U}$ & $\mathrm{U}$ & $\mathrm{U}$ \\
\hline Chloroform & $\mathrm{U}$ & $\mathrm{U}$ & $\mathrm{U}$ & $\mathrm{U}$ \\
\hline Chloromethane & $\mathrm{U}$ & $\mathrm{U}$ & $\mathrm{U}$ & $\mathrm{U}$ \\
\hline
\end{tabular}


Table C-1. (continued).

\begin{tabular}{|c|c|c|c|c|}
\hline \multirow[b]{2}{*}{ Analyzed Constituents } & \multicolumn{4}{|c|}{ Sample Dates } \\
\hline & $\begin{array}{c}\text { November } \\
2000 \\
\end{array}$ & $\begin{array}{c}\text { January } \\
2001 \\
\end{array}$ & $\begin{array}{c}\text { February } \\
2001 \\
\end{array}$ & $\begin{array}{c}\text { March } \\
2001 \\
\end{array}$ \\
\hline CLP-List Volatile Organic Compounds & $(\mathrm{mg} / \mathrm{L})$ & $(\mathrm{mg} / \mathrm{L})$ & $(\mathrm{mg} / \mathrm{L})$ & $(\mathrm{mg} / \mathrm{L})$ \\
\hline Dibromochloromethane & $\mathrm{U}$ & $\mathrm{U}$ & $\mathrm{U}$ & $\mathrm{U}$ \\
\hline Ethylbenzene & $\mathrm{U}$ & $\mathrm{U}$ & $\mathrm{U}$ & $\mathrm{U}$ \\
\hline Methylene Chloride & $\mathrm{U}$ & $\mathrm{U}$ & $\mathrm{U}$ & $\mathrm{U}$ \\
\hline Styrene & $\mathrm{U}$ & $\mathrm{U}$ & $\mathrm{U}$ & $\mathrm{U}$ \\
\hline Tetrachloroethene & $\mathrm{U}$ & $\mathrm{U}$ & $\mathrm{U}$ & $\mathrm{U}$ \\
\hline Toluene & $\mathrm{U}$ & $\mathrm{U}$ & $\mathrm{U}$ & $\mathrm{U}$ \\
\hline Trichloroethene & $\mathrm{U}$ & $\mathrm{U}$ & $\mathrm{U}$ & $\mathrm{U}$ \\
\hline Vinyl Chloride & $\mathrm{U}$ & $\mathrm{U}$ & $\mathrm{U}$ & $\mathrm{U}$ \\
\hline Xylenes & $\mathrm{U}$ & $\mathrm{U}$ & $\mathrm{U}$ & $\mathrm{U}$ \\
\hline Cis-1,3-Dichloropropene & $\mathrm{U}$ & $\mathrm{U}$ & $\mathrm{U}$ & $\mathrm{U}$ \\
\hline Trans-1,3-Dichloropropene & $\mathrm{U}$ & $\mathrm{U}$ & $\mathrm{U}$ & $\mathrm{U}$ \\
\hline Alpha emitters & $(\mathrm{pCi} / \mathrm{L})$ & $(\mathrm{pCi} / \mathrm{L})$ & $(\mathrm{pCi} / \mathrm{L})$ & $(\mathrm{pCi} / \mathrm{L})$ \\
\hline Americium 241 & $\begin{array}{c}1.12 \mathrm{E}-02 \pm \\
5.63 \mathrm{E}-03 \mathrm{UJ}\end{array}$ & $\mathrm{U}$ & $\begin{array}{c}1.54 \mathrm{E}-02 \pm \\
6.94 \mathrm{E}-03 \mathrm{~J}\end{array}$ & $\mathrm{U}$ \\
\hline Neptunium-237 & $\mathrm{U}$ & $\begin{array}{l}1.94 \mathrm{E}-02 \pm \\
8.96 \mathrm{E}-03 \mathrm{UJ}\end{array}$ & $\mathrm{U}$ & $\mathrm{U}$ \\
\hline Plutonium-238 & $\begin{array}{c}2.60 \mathrm{E}-02 \pm \\
1.09 \mathrm{E}-02 \mathrm{~J}\end{array}$ & $\mathrm{U}$ & $\mathrm{U}$ & $\mathrm{U}$ \\
\hline Plutonium 239/240 & $\mathrm{U}$ & $\mathrm{U}$ & $\mathrm{U}$ & $\mathrm{U}$ \\
\hline Uranium-233/234 & $\begin{array}{c}1.35 \mathrm{E}+00 \pm \\
1.61 \mathrm{E}-01\end{array}$ & $\begin{array}{c}1.15 \mathrm{E}+00 \pm \\
1.21 \mathrm{E}-01\end{array}$ & $\begin{array}{c}1.41 \mathrm{E}+00 \pm \\
2.00 \mathrm{E}-01\end{array}$ & $\begin{array}{c}1.42 \mathrm{E}+00 \pm \\
1.13 \mathrm{E}-01\end{array}$ \\
\hline Uranium-235/236 & $\mathrm{U}$ & $\mathrm{U}$ & $\mathrm{U}$ & $\begin{array}{c}5.75 \mathrm{E}-02 \pm \\
1.14 \mathrm{E}-02\end{array}$ \\
\hline Uranium-238 & $\begin{array}{c}6.81 \mathrm{E}-01 \pm \\
9.94 \mathrm{E}-02 \\
\end{array}$ & $\begin{array}{c}6.01 \mathrm{E}-01 \pm \\
7.59 \mathrm{E}-02 \\
\end{array}$ & $\begin{array}{c}8.09 \mathrm{E}-01 \pm \\
1.39 \mathrm{E}-01 \\
\end{array}$ & $\begin{array}{c}7.49 \mathrm{E}-01 \pm \\
6.51 \mathrm{E}-02 \\
\end{array}$ \\
\hline Gamma Emitters & $(\mathrm{pCi} / \mathrm{L})$ & $(\mathrm{pCi} / \mathrm{L})$ & $(\mathrm{pCi} / \mathrm{L})$ & $(\mathrm{pCi} / \mathrm{L})$ \\
\hline Americium 241 & $\mathrm{U}$ & $\mathrm{U}$ & $\mathrm{U}$ & $\mathrm{U}$ \\
\hline Antimony-125 & $\mathrm{U}$ & $\mathrm{U}$ & $\mathrm{U}$ & $\mathrm{U}$ \\
\hline Cerium 144 & $\mathrm{U}$ & $\mathrm{U}$ & $\mathrm{U}$ & $\mathrm{U}$ \\
\hline Cesium 134 & $\mathrm{U}$ & $\mathrm{U}$ & $\mathrm{U}$ & $\mathrm{U}$ \\
\hline Cesium 137 & $\mathrm{U}$ & $\mathrm{U}$ & $\mathrm{U}$ & $\mathrm{U}$ \\
\hline Cobalt-58 & $\mathrm{U}$ & $\mathrm{U}$ & $\mathrm{U}$ & $\mathrm{U}$ \\
\hline Cobalt- 60 & $\mathrm{U}$ & $\mathrm{U}$ & $\mathrm{U}$ & $\mathrm{U}$ \\
\hline Europium-152 & $\mathrm{U}$ & $\mathrm{U}$ & $\mathrm{U}$ & $\mathrm{U}$ \\
\hline Europium-154 & $\mathrm{U}$ & $\mathrm{U}$ & $\mathrm{U}$ & $\mathrm{U}$ \\
\hline Europium-155 & $\mathrm{U}$ & $\mathrm{U}$ & $\mathrm{U}$ & $\mathrm{U}$ \\
\hline
\end{tabular}


Table C-1. (continued).

\begin{tabular}{|c|c|c|c|c|}
\hline \multirow[b]{2}{*}{ Analyzed Constituents } & \multicolumn{4}{|c|}{ Sample Dates } \\
\hline & $\begin{array}{c}\text { November } \\
2000 \\
\end{array}$ & $\begin{array}{c}\text { January } \\
2001 \\
\end{array}$ & $\begin{array}{c}\text { February } \\
2001 \\
\end{array}$ & $\begin{array}{c}\text { March } \\
2001 \\
\end{array}$ \\
\hline Gamma Emitters & $(\mathrm{pCi} / \mathrm{L})$ & $(\mathrm{pCi} / \mathrm{L})$ & $(\mathrm{pCi} / \mathrm{L})$ & $(\mathrm{pCi} / \mathrm{L})$ \\
\hline Manganese-54 & $\mathrm{U}$ & $\mathrm{U}$ & $\mathrm{U}$ & $\mathrm{U}$ \\
\hline Niobium-95 & $\mathrm{U}$ & $\mathrm{U}$ & $\mathrm{U}$ & $\begin{array}{l}1.68 \mathrm{E}+00 \pm \\
7.88 \mathrm{E}-01 \mathrm{UJ}\end{array}$ \\
\hline Potassium-40 & $\begin{array}{r}2.81 \mathrm{E}+01 \pm \\
1.07 \mathrm{E}+01 \mathrm{~J}\end{array}$ & $\begin{array}{c}3.06 \mathrm{E}+01 \pm \\
9.60 \mathrm{E}+00\end{array}$ & $\mathrm{U}$ & $\begin{array}{c}1.64 \mathrm{E}+01 \pm \\
7.34 \mathrm{E}+00 \mathrm{UJ}\end{array}$ \\
\hline Radium-226 & $\mathrm{U}$ & $\begin{array}{l}3.71 \mathrm{E}+00 \pm \\
1.43 \mathrm{E}+00 \mathrm{UJ}\end{array}$ & $\mathrm{U}$ & $\mathrm{U}$ \\
\hline Ruthenium-103 & $\mathrm{U}$ & $\mathrm{U}$ & $\mathrm{U}$ & $\begin{array}{l}1.81 \mathrm{E}+00 \pm \\
7.85 \mathrm{E}-01 \mathrm{UJ}\end{array}$ \\
\hline Ruthenium-106 & $\mathrm{U}$ & $\mathrm{U}$ & $\mathrm{U}$ & $\mathrm{U}$ \\
\hline Silver-108m & $\mathrm{U}$ & $\mathrm{U}$ & $\mathrm{U}$ & $\mathrm{U}$ \\
\hline Silver-110m & $\mathrm{U}$ & $\mathrm{U}$ & $\mathrm{U}$ & $\mathrm{U}$ \\
\hline Uranium-235 & $\mathrm{U}$ & $\mathrm{U}$ & $\mathrm{U}$ & $\mathrm{U}$ \\
\hline Zinc-65 & $\mathrm{U}$ & $\mathrm{U}$ & $\mathrm{U}$ & $\mathrm{U}$ \\
\hline Zirconium-95 & $\mathrm{U}$ & $\mathrm{U}$ & $\mathrm{U}$ & $\mathrm{U}$ \\
\hline Isotopic Analyses & $(\mathrm{pCi} / \mathrm{L})$ & $(\mathrm{pCi} / \mathrm{L})$ & $(\mathrm{pCi} / \mathrm{L})$ & $(\mathrm{pCi} / \mathrm{L})$ \\
\hline Carbon-14 & $\mathrm{U}$ & $\begin{array}{l}2.44 \mathrm{E}+00 \pm \\
7.44 \mathrm{E}-01 \mathrm{UJ}\end{array}$ & $\mathrm{U}$ & $\mathrm{U}$ \\
\hline Gross Alpha & $\begin{array}{c}8.78 \mathrm{E}-01 \pm \\
3.65 \mathrm{E}-01 \mathrm{UJ}\end{array}$ & $\begin{array}{c}8.66 \mathrm{E}-01 \pm \\
2.77 \mathrm{E}-01\end{array}$ & $\begin{array}{l}2.23 \mathrm{E}+00 \pm \\
8.71 \mathrm{E}-01 \mathrm{UJ}\end{array}$ & $\begin{array}{c}1.98 \mathrm{E}+00 \pm \\
6.21 \mathrm{E}-01\end{array}$ \\
\hline Gross Beta & $\begin{array}{l}2.02 \mathrm{E}+00 \pm \\
7.03 \mathrm{E}-01 \mathrm{UJ}\end{array}$ & $\begin{array}{c}2.73 \mathrm{E}+00 \pm \\
3.45 \mathrm{E}-01\end{array}$ & $\mathrm{U}$ & $\begin{array}{l}1.53 \mathrm{E}+00 \pm \\
6.52 \mathrm{E}-01 \mathrm{UJ}\end{array}$ \\
\hline Iodine-129 & $\mathrm{U}$ & $\mathrm{U}$ & $\mathrm{U}$ & $\mathrm{U}$ \\
\hline Radium-228 & NS & NS & NS & NS \\
\hline Strontium-90 & $\mathrm{U}$ & $\mathrm{U}$ & $\mathrm{U}$ & $\mathrm{U}$ \\
\hline Technicium-99 & $\mathrm{U}$ & $\mathrm{U}$ & $\mathrm{U}$ & $\mathrm{U}$ \\
\hline Tritium & $\mathrm{U}$ & $\mathrm{U}$ & $\mathrm{U}$ & $\mathrm{U}$ \\
\hline
\end{tabular}


Table C-2 Baseline groundwater data for Well ICPP-MON-A-165.

\begin{tabular}{|c|c|c|c|c|c|}
\hline \multirow[b]{2}{*}{ Analyzed Constituents } & \multicolumn{5}{|c|}{ Sample Dates } \\
\hline & $\begin{array}{c}\text { November } \\
2000\end{array}$ & $\begin{array}{c}\text { January } \\
2001 \\
\end{array}$ & $\begin{array}{c}\text { February } \\
2001\end{array}$ & $\begin{array}{c}\text { March } \\
2001\end{array}$ & $\begin{array}{c}\text { March } \\
\text { Duplicate }\end{array}$ \\
\hline Miscellaneous Inorganics & $(\mathrm{mg} / \mathrm{L})$ & $(\mathrm{mg} / \mathrm{L})$ & $(\mathrm{mg} / \mathrm{L})$ & $(\mathrm{mg} / \mathrm{L})$ & $(\mathrm{mg} / \mathrm{L})$ \\
\hline Alkalinity & 155 & 153 & 154 & 153 & 154 \\
\hline Biochemical Oxygen Demand & $\mathrm{U}$ & $\mathrm{U}$ & $\mathrm{U}$ & NS & NS \\
\hline Bromide & NS & NS & $\mathrm{U}$ & $\mathrm{U}$ & $\mathrm{U}$ \\
\hline Chloride & NS & NS & 7.7 & 6.0 & 8.0 \\
\hline Chromium hexavalent & NS & $\mathrm{R}$ & $\mathrm{U}$ & NS & NS \\
\hline Fecal Coliform (COL/DL) & None & None & None & None & None \\
\hline Total Coliform (COL/DL) & None & 4200 & 24 & 9J & None \\
\hline Fluoride & NS & NS & 0.19 & $0.13 \mathrm{E}$ & 0.15 \\
\hline Nitrate & $0.66 \mathrm{E}$ & 0.65 & 0.64 & $1.1 \mathrm{EJ}$ & $0.66 \mathrm{~J}$ \\
\hline Nitrite & $\mathrm{U}$ & $\mathrm{U}$ & 0.01 & $\mathrm{U}$ & $\mathrm{U}$ \\
\hline Nitrogen, Ammonia (as N) & $\mathrm{U}$ & $\mathrm{U}$ & $\mathrm{U}$ & $\mathrm{U}$ & $\mathrm{U}$ \\
\hline Nitrogen, Nitrate-Nitrite (as N) & $0.671 \mathrm{~J}$ & 0.731 & 0.707 & 0.690 & 0.696 \\
\hline Phosphate & NS & NS & $\mathrm{U}$ & $\mathrm{U}$ & $\mathrm{U}$ \\
\hline Phosphorous, Total (as P) & 0.0263 & 0.039 & 0.266 & 0.0194 & 0.0231 \\
\hline Sulfate & NS & NS & 28.9 & 30.5 & 30.4 \\
\hline Total Dissolved Solids & 232 & 244 & 230 & 249 & 247 \\
\hline Total Kjeldahl Nitrogen & 0.511 & $\mathrm{U}$ & 0.241 & $0.157 \mathrm{~J}$ & $0.1 \mathrm{UJ}$ \\
\hline TAL Metals & $(\mathrm{mg} / \mathrm{L})$ & $(\mathrm{mg} / \mathrm{L})$ & $(\mathrm{mg} / \mathrm{L})$ & $(\mathrm{mg} / \mathrm{L})$ & $(\mathrm{mg} / \mathrm{L})$ \\
\hline Aluminum & $\mathrm{U}$ & $\mathrm{U}$ & $\mathrm{U}$ & $\mathrm{U}$ & $\mathrm{U}$ \\
\hline Antimony & $\mathrm{U}$ & $\mathrm{U}$ & $\mathrm{U}$ & $\mathrm{U}$ & $\mathrm{U}$ \\
\hline Arsenic & 0.0052 & $\mathrm{U}$ & $\mathrm{U}$ & $\mathrm{U}$ & $\mathrm{U}$ \\
\hline Barium & 0.0729 & 0.0702 & 0.0703 & $0.073 \mathrm{~B}$ & $0.0731 \mathrm{~B}$ \\
\hline Beryllium & $\mathrm{U}$ & $\mathrm{U}$ & $\mathrm{U}$ & $\mathrm{U}$ & $\mathrm{U}$ \\
\hline Boron & 0.0384 & $\mathrm{U}$ & $\mathrm{U}$ & $\mathrm{U}$ & $\mathrm{U}$ \\
\hline Cadmium & $\mathrm{U}$ & $\mathrm{U}$ & $\mathrm{U}$ & $\mathrm{U}$ & $\mathrm{U}$ \\
\hline Calcium & 46.9 & 44.5 & 45.8 & 46.8 & 46.4 \\
\hline Chromium & 0.0088 & 0.0068 & 0.0062 & $0.0079 \mathrm{~B}$ & $0.0081 \mathrm{~B}$ \\
\hline Cobalt & $\mathrm{U}$ & $\mathrm{U}$ & $\mathrm{U}$ & $\mathrm{U}$ & $\mathrm{U}$ \\
\hline Copper & $\mathrm{U}$ & $\mathrm{U}$ & $\mathrm{U}$ & $\mathrm{U}$ & $\mathrm{U}$ \\
\hline Iron & $0.0697 \mathrm{~B}$ & $\mathrm{U}$ & $\mathrm{U}$ & $\mathrm{U}$ & $\mathrm{U}$ \\
\hline Lead & $\mathrm{U}$ & $\mathrm{U}$ & $\mathrm{U}$ & $\mathrm{U}$ & $\mathrm{U}$ \\
\hline Lithium & $\mathrm{U}$ & $\mathrm{U}$ & $\mathrm{U}$ & $\mathrm{U}$ & $\mathrm{U}$ \\
\hline Magnesium & 13.9 & 13.1 & 13.4 & 13.8 & 13.7 \\
\hline
\end{tabular}


Table C-2. (continued).

\begin{tabular}{|c|c|c|c|c|c|}
\hline \multirow[b]{2}{*}{ Analyzed Constituents } & \multicolumn{5}{|c|}{ Sample Dates } \\
\hline & $\begin{array}{c}\text { November } \\
2000\end{array}$ & $\begin{array}{c}\text { January } \\
2001\end{array}$ & $\begin{array}{c}\text { February } \\
2001\end{array}$ & $\begin{array}{c}\text { March } \\
2001 \\
\end{array}$ & $\begin{array}{c}\text { March } \\
\text { Duplicate }\end{array}$ \\
\hline TAL Metals & $(\mathrm{mg} / \mathrm{L})$ & $(\mathrm{mg} / \mathrm{L})$ & $(\mathrm{mg} / \mathrm{L})$ & $(\mathrm{mg} / \mathrm{L})$ & $(\mathrm{mg} / \mathrm{L})$ \\
\hline Manganese & $\mathrm{U}$ & $\mathrm{U}$ & $\mathrm{U}$ & $\mathrm{U}$ & $\mathrm{U}$ \\
\hline Mercury & $\mathrm{U}$ & $\mathrm{U}$ & $\mathrm{U}$ & $\mathrm{U}$ & $\mathrm{U}$ \\
\hline Nickel & $\mathrm{U}$ & $\mathrm{U}$ & $\mathrm{U}$ & $\mathrm{U}$ & $\mathrm{U}$ \\
\hline Potassium & $2.08 \mathrm{~B}$ & $2.1 \mathrm{~B}$ & $1.98 \mathrm{~B}$ & $2.09 \mathrm{~B}$ & $2.08 \mathrm{~B}$ \\
\hline Selenium & $\mathrm{U}$ & $\mathrm{U}$ & $\mathrm{U}$ & $\mathrm{U}$ & $\mathrm{U}$ \\
\hline Silicon & 10.5 & 9.66 & 9.76 & 1.11 & 1.1 \\
\hline Silver & $\mathrm{U}$ & $\mathrm{U}$ & $\mathrm{U}$ & $\mathrm{U}$ & $\mathrm{U}$ \\
\hline Sodium & 8.03 & 7.77 & 7.9 & 7.6 & 7.7 \\
\hline Strontium & 0.266 & 0.249 & 0.257 & 0.26 & 0.259 \\
\hline Thallium & $\mathrm{U}$ & $\mathrm{U}$ & $\mathrm{U}$ & $\mathrm{U}$ & $\mathrm{U}$ \\
\hline Vanadium & $\mathrm{U}$ & $\mathrm{U}$ & 0.0051 & $\mathrm{U}$ & $\mathrm{U}$ \\
\hline Zinc & 0.0106 & 0.0077 & $\mathrm{U}$ & $\mathrm{U}$ & $\mathrm{U}$ \\
\hline CLP-List Volatile Organic Compounds & $(\mathrm{mg} / \mathrm{L})$ & $(\mathrm{mg} / \mathrm{L})$ & $(\mathrm{mg} / \mathrm{L})$ & $(\mathrm{mg} / \mathrm{L})$ & $(\mathrm{mg} / \mathrm{L})$ \\
\hline 1,1,1-Trichloroethane & $\mathrm{U}$ & $\mathrm{U}$ & $\mathrm{U}$ & $\mathrm{U}$ & $\mathrm{U}$ \\
\hline 1,1,2,2-Tetrachloroethane & $\mathrm{U}$ & $\mathrm{U}$ & $\mathrm{U}$ & $\mathrm{U}$ & $\mathrm{U}$ \\
\hline 1,1,2-Trichloroethane & $\mathrm{U}$ & $\mathrm{U}$ & $\mathrm{U}$ & $\mathrm{U}$ & $\mathrm{U}$ \\
\hline 1,1-Dichloroethane & $\mathrm{U}$ & $\mathrm{U}$ & $\mathrm{U}$ & $\mathrm{U}$ & $\mathrm{U}$ \\
\hline 1,1-Dichloroethene & $\mathrm{U}$ & $\mathrm{U}$ & $\mathrm{U}$ & $\mathrm{U}$ & $\mathrm{U}$ \\
\hline 1,2-Dichloroethane & $\mathrm{U}$ & $\mathrm{U}$ & $\mathrm{U}$ & $\mathrm{U}$ & $\mathrm{U}$ \\
\hline 1,2-Dichloroethene (Total) & $\mathrm{U}$ & $\mathrm{U}$ & $\mathrm{U}$ & $\mathrm{U}$ & $\mathrm{U}$ \\
\hline 1,2-Dichloropropane & $\mathrm{U}$ & $\mathrm{U}$ & $\mathrm{U}$ & $\mathrm{U}$ & $\mathrm{U}$ \\
\hline 2-Butanone & $\mathrm{R}$ & $\mathrm{R}$ & $\mathrm{U}$ & $\mathrm{U}$ & $\mathrm{U}$ \\
\hline 2-Hexanone & $\mathrm{U}$ & $\mathrm{U}$ & $\mathrm{U}$ & $\mathrm{U}$ & $\mathrm{U}$ \\
\hline 4-Methyl-2-pentanone & $\mathrm{U}$ & $\mathrm{U}$ & $\mathrm{U}$ & $\mathrm{U}$ & $\mathrm{U}$ \\
\hline Acetone & $\mathrm{U}$ & $\mathrm{U}$ & $\mathrm{U}$ & $\mathrm{U}$ & $\mathrm{U}$ \\
\hline Benzene & $\mathrm{U}$ & $\mathrm{U}$ & $\mathrm{U}$ & $\mathrm{U}$ & $\mathrm{U}$ \\
\hline Bromodichloromethane & $\mathrm{U}$ & $\mathrm{U}$ & $\mathrm{U}$ & $\mathrm{U}$ & $\mathrm{U}$ \\
\hline Bromoform & $\mathrm{U}$ & $\mathrm{U}$ & $\mathrm{U}$ & $\mathrm{U}$ & $\mathrm{U}$ \\
\hline Bromomethane & $\mathrm{U}$ & $\mathrm{U}$ & $\mathrm{U}$ & $\mathrm{U}$ & $\mathrm{U}$ \\
\hline Carbon disulfide & $\mathrm{U}$ & $\mathrm{U}$ & $\mathrm{U}$ & $\mathrm{U}$ & $\mathrm{U}$ \\
\hline Carbon tetrachloride & $\mathrm{U}$ & $\mathrm{U}$ & $\mathrm{U}$ & $\mathrm{U}$ & $\mathrm{U}$ \\
\hline Chlorobenzene & $\mathrm{U}$ & $\mathrm{U}$ & $\mathrm{U}$ & $\mathrm{U}$ & $\mathrm{U}$ \\
\hline Chloroethane & $\mathrm{U}$ & $\mathrm{U}$ & $\mathrm{U}$ & $\mathrm{U}$ & $\mathrm{U}$ \\
\hline Chloroform & $\mathrm{U}$ & $\mathrm{U}$ & $\mathrm{U}$ & $\mathrm{U}$ & $\mathrm{U}$ \\
\hline Chloromethane & $\mathrm{U}$ & $\mathrm{U}$ & $\mathrm{U}$ & $\mathrm{U}$ & $\mathrm{U}$ \\
\hline
\end{tabular}


Table C-2. (continued).

\begin{tabular}{|c|c|c|c|c|c|}
\hline \multirow[b]{2}{*}{ Analyzed Constituents } & \multicolumn{5}{|c|}{ Sample Dates } \\
\hline & $\begin{array}{c}\text { November } \\
2000\end{array}$ & $\begin{array}{c}\text { January } \\
2001\end{array}$ & $\begin{array}{c}\text { February } \\
2001\end{array}$ & $\begin{array}{c}\text { March } \\
2001\end{array}$ & $\begin{array}{c}\text { March } \\
\text { Duplicate }\end{array}$ \\
\hline CLP-List Volatile Organic Compounds & $(\mathrm{mg} / \mathrm{L})$ & $(\mathrm{mg} / \mathrm{L})$ & $(\mathrm{mg} / \mathrm{L})$ & $(\mathrm{mg} / \mathrm{L})$ & $(\mathrm{mg} / \mathrm{L})$ \\
\hline Dibromochloromethane & $\mathrm{U}$ & $\mathrm{U}$ & $\mathrm{U}$ & $\mathrm{U}$ & $\mathrm{U}$ \\
\hline Ethylbenzene & $\mathrm{U}$ & $\mathrm{U}$ & $\mathrm{U}$ & $\mathrm{U}$ & $\mathrm{U}$ \\
\hline Methylene Chloride & $\mathrm{U}$ & $\mathrm{U}$ & $\mathrm{U}$ & $1 \mathrm{~J}$ & $\mathrm{U}$ \\
\hline Styrene & $\mathrm{U}$ & $\mathrm{U}$ & $\mathrm{U}$ & $\mathrm{U}$ & $\mathrm{U}$ \\
\hline Tetrachloroethene & $\mathrm{U}$ & $\mathrm{U}$ & $\mathrm{U}$ & $\mathrm{U}$ & $\mathrm{U}$ \\
\hline Toluene & $\mathrm{U}$ & $\mathrm{U}$ & $\mathrm{U}$ & $\mathrm{U}$ & $\mathrm{U}$ \\
\hline Trichloroethene & $\mathrm{U}$ & $\mathrm{U}$ & $\mathrm{U}$ & $\mathrm{U}$ & $\mathrm{U}$ \\
\hline Vinyl Chloride & $\mathrm{U}$ & $\mathrm{U}$ & $\mathrm{U}$ & $\mathrm{U}$ & $\mathrm{U}$ \\
\hline Xylenes & $\mathrm{U}$ & $\mathrm{U}$ & $\mathrm{U}$ & $\mathrm{U}$ & $\mathrm{U}$ \\
\hline Cis-1,3-Dichloropropene & $\mathrm{U}$ & $\mathrm{U}$ & $\mathrm{U}$ & $\mathrm{U}$ & $\mathrm{U}$ \\
\hline Trans-1,3-Dichloropropene & $\mathrm{U}$ & $\mathrm{U}$ & $\mathrm{U}$ & $\mathrm{U}$ & $\mathrm{U}$ \\
\hline Alpha emitters & $(\mathrm{pCi} / \mathrm{L})$ & $(\mathrm{pCi} / \mathrm{L})$ & $(\mathrm{pCi} / \mathrm{L})$ & $(\mathrm{pCi} / \mathrm{L})$ & $(\mathrm{pCi} / \mathrm{L})$ \\
\hline Americium 241 & $\mathrm{U}$ & $\mathrm{U}$ & $\begin{array}{c}3.10 \mathrm{E}-02 \pm \\
1.09 \mathrm{E}-02 \mathrm{~J}\end{array}$ & $\begin{array}{l}1.88 \mathrm{E}-02 \pm \\
7.20 \mathrm{E}-03 \mathrm{~J}\end{array}$ & $\begin{array}{c}2.09 \mathrm{E}-02 \pm \\
7.79 \mathrm{E}-03 \mathrm{~J}\end{array}$ \\
\hline Neptunium-237 & $\mathrm{U}$ & $\mathrm{U}$ & $\mathrm{U}$ & $\mathrm{U}$ & $\mathrm{U}$ \\
\hline Plutonium-238 & $\mathrm{U}$ & $\mathrm{U}$ & $\mathrm{U}$ & $\mathrm{U}$ & $\mathrm{U}$ \\
\hline Plutonium-239/240 & $\mathrm{U}$ & $\mathrm{U}$ & $\mathrm{U}$ & $\mathrm{U}$ & $\mathrm{U}$ \\
\hline Uranium-233/234 & $\begin{array}{c}1.36 \mathrm{E}+00 \pm \\
1.54 \mathrm{E}-01\end{array}$ & $\begin{array}{c}1.28 \mathrm{E}+00 \pm \\
1.27 \mathrm{E}-01\end{array}$ & $\begin{array}{c}1.10 \mathrm{E}+00 \pm \\
1.56 \mathrm{E}-01\end{array}$ & $\begin{array}{c}1.29 \mathrm{E}+00 \pm \\
1.17 \mathrm{E}-01\end{array}$ & $\begin{array}{c}1.29 \mathrm{E}+00 \pm \\
1.22 \mathrm{E}-01\end{array}$ \\
\hline Uranium-235/236 & $\mathrm{U}$ & $\mathrm{U}$ & $\mathrm{U}$ & $\begin{array}{c}3.68 \mathrm{E}-02 \pm \\
1.34 \mathrm{E}-02 \mathrm{~J}\end{array}$ & $\begin{array}{c}4.25 \mathrm{E}-02 \pm \\
1.38 \mathrm{E}-02\end{array}$ \\
\hline Uranium-238 & $\begin{array}{c}4.41 \mathrm{E}-01 \pm \\
7.02 \mathrm{E}-02 \\
\end{array}$ & $\begin{array}{c}6.97 \mathrm{E}-01 \pm \\
8.02 \mathrm{E}-02 \\
\end{array}$ & $\begin{array}{c}4.70 \mathrm{E}-01 \pm \\
9.07 \mathrm{E}-02 \\
\end{array}$ & $\begin{array}{c}5.20 \mathrm{E}-01 \pm \\
5.80 \mathrm{E}-02 \\
\end{array}$ & $\begin{array}{c}6.36 \mathrm{E}-01 \pm \\
7.04 \mathrm{E}-02 \\
\end{array}$ \\
\hline Gamma Emitters & $(\mathrm{pCi} / \mathrm{L})$ & $(\mathrm{pCi} / \mathrm{L})$ & $(\mathrm{pCi} / \mathrm{L})$ & $(\mathrm{pCi} / \mathrm{L})$ & $(\mathrm{pCi} / \mathrm{L})$ \\
\hline Americium 241 & $\mathrm{U}$ & $\mathrm{U}$ & $\mathrm{U}$ & $\begin{array}{l}1.27 \mathrm{E}+01 \pm \\
6.31 \mathrm{E}+00 \mathrm{UJ}\end{array}$ & $\mathrm{U}$ \\
\hline Antimony-125 & $\mathrm{U}$ & $\mathrm{U}$ & $\mathrm{U}$ & $\mathrm{U}$ & $\begin{array}{l}4.29 \mathrm{E}+00 \pm \\
1.64 \mathrm{E}+00 \mathrm{UJ}\end{array}$ \\
\hline Cerium-144 & $\mathrm{U}$ & $\mathrm{U}$ & $\mathrm{U}$ & $\mathrm{U}$ & $\mathrm{U}$ \\
\hline Cesium-134 & $\mathrm{U}$ & $\mathrm{U}$ & $\mathrm{U}$ & $\mathrm{U}$ & $\mathrm{U}$ \\
\hline Cesium 137 & $\mathrm{U}$ & $\mathrm{U}$ & $\mathrm{U}$ & $\mathrm{U}$ & $\mathrm{U}$ \\
\hline Cobalt-58 & $\mathrm{U}$ & $\mathrm{U}$ & $\mathrm{U}$ & $\mathrm{U}$ & $\mathrm{U}$ \\
\hline Cobalt-60 & $\mathrm{U}$ & $\mathrm{U}$ & $\mathrm{U}$ & $\mathrm{U}$ & $\begin{array}{l}2.04 \mathrm{E}+00 \pm \\
7.13 \mathrm{E}-01 \mathrm{UJ}\end{array}$ \\
\hline Europium-152 & $\mathrm{U}$ & $\mathrm{U}$ & $\mathrm{U}$ & $\mathrm{U}$ & U \\
\hline Europium-154 & $\mathrm{U}$ & $\mathrm{U}$ & $\mathrm{U}$ & $\mathrm{U}$ & $\mathrm{U}$ \\
\hline
\end{tabular}


Table C-2. (continued).

\begin{tabular}{|c|c|c|c|c|c|}
\hline \multirow[b]{2}{*}{ Analyzed Constituents } & \multicolumn{5}{|c|}{ Sample Dates } \\
\hline & $\begin{array}{c}\text { November } \\
2000\end{array}$ & $\begin{array}{c}\text { January } \\
2001\end{array}$ & $\begin{array}{c}\text { February } \\
2001\end{array}$ & $\begin{array}{c}\text { March } \\
2001\end{array}$ & $\begin{array}{c}\text { March } \\
\text { Duplicate }\end{array}$ \\
\hline Gamma Emitters & $(\mathrm{pCi} / \mathrm{L})$ & $(\mathrm{pCi} / \mathrm{L})$ & $(\mathrm{pCi} / \mathrm{L})$ & $(\mathrm{pCi} / \mathrm{L})$ & $(\mathrm{pCi} / \mathrm{L})$ \\
\hline Europium-155 & $\mathrm{U}$ & $\mathrm{U}$ & $\mathrm{U}$ & $\mathrm{U}$ & $\mathrm{U}$ \\
\hline Manganese-54 & $\mathrm{U}$ & $\mathrm{U}$ & $\mathrm{U}$ & $\mathrm{U}$ & $\mathrm{U}$ \\
\hline Niobium-95 & $\mathrm{U}$ & $\mathrm{U}$ & $\mathrm{U}$ & $\mathrm{U}$ & $\mathrm{U}$ \\
\hline Potassium-40 & $\begin{array}{l}2.38 \mathrm{E}+01 \pm \\
6.75 \mathrm{E}+00 \mathrm{UJ}\end{array}$ & $\begin{array}{c}3.75 \mathrm{E}+01 \pm \\
1.22 \mathrm{E}+01\end{array}$ & $\mathrm{U}$ & $\mathrm{U}$ & $\mathrm{U}$ \\
\hline Radium-226 & $\mathrm{U}$ & $\mathrm{U}$ & $\mathrm{U}$ & $\begin{array}{l}3.32 \mathrm{E}+00 \pm \\
1.28 \mathrm{E}+00 \mathrm{UJ}\end{array}$ & $\begin{array}{c}2.28 \mathrm{E}+01 \pm \\
7.84 \mathrm{E}+00 \mathrm{UJ}\end{array}$ \\
\hline Ruthenium-103 & $\mathrm{U}$ & $\mathrm{U}$ & $\mathrm{U}$ & $\mathrm{U}$ & $\mathrm{U}$ \\
\hline Ruthenium-106 & $\mathrm{U}$ & $\begin{array}{l}1.57 \mathrm{E}+01 \pm \\
6.85 \mathrm{E}+00 \mathrm{UJ}\end{array}$ & $\mathrm{U}$ & $\mathrm{U}$ & $\mathrm{U}$ \\
\hline Silver-108m & $\mathrm{U}$ & $\mathrm{U}$ & $\mathrm{U}$ & $\mathrm{U}$ & $\mathrm{U}$ \\
\hline Silver-110m & $\mathrm{U}$ & $\mathrm{U}$ & $\mathrm{U}$ & $\mathrm{U}$ & $\mathrm{U}$ \\
\hline Uranium-235 & $\mathrm{U}$ & $\mathrm{U}$ & $\mathrm{U}$ & $\mathrm{U}$ & $\mathrm{U}$ \\
\hline Zinc-65 & $\mathrm{U}$ & $\mathrm{U}$ & $\mathrm{U}$ & $\mathrm{U}$ & $\mathrm{U}$ \\
\hline Zirconium-95 & $\mathrm{U}$ & $\mathrm{U}$ & $\mathrm{U}$ & $\mathrm{U}$ & $\mathrm{U}$ \\
\hline Isotopic Analyses & $(\mathrm{pCi} / \mathrm{L})$ & $(\mathrm{pCi} / \mathrm{L})$ & $(\mathrm{pCi} / \mathrm{L})$ & $(\mathrm{pCi} / \mathrm{L})$ & $(\mathrm{pCi} / \mathrm{L})$ \\
\hline Carbon-14 & $\begin{array}{c}3.33 \mathrm{E}+00 \pm \\
8.02 \mathrm{E}-01\end{array}$ & $\begin{array}{l}2.66 \mathrm{E}+00 \pm \\
7.34 \mathrm{E}-01 \mathrm{UJ}\end{array}$ & $\mathrm{U}$ & $\mathrm{U}$ & $\begin{array}{c}4.51 \mathrm{E}+00 \pm \\
9.52 \mathrm{E}-01\end{array}$ \\
\hline Gross Alpha & $\begin{array}{l}5.60 \mathrm{E}+00 \pm \\
2.23 \mathrm{E}+00 \mathrm{UJ}\end{array}$ & $\begin{array}{c}1.74 \mathrm{E}+00 \pm \\
3.18 \mathrm{E}-01\end{array}$ & $\begin{array}{l}1.61 \mathrm{E}+00 \pm \\
7.79 \mathrm{E}-01 \mathrm{UJ}\end{array}$ & $\begin{array}{c}2.04 \mathrm{E}+00 \pm \\
5.78 \mathrm{E}-01\end{array}$ & $\begin{array}{c}2.18 \mathrm{E}+00 \pm \\
6.15 \mathrm{E}-01\end{array}$ \\
\hline Gross Beta & $\begin{array}{c}9.85 \mathrm{E}+00 \pm \\
1.56 \mathrm{E}+00 \mathrm{~J}\end{array}$ & $\begin{array}{c}2.45 \mathrm{E}+00 \pm \\
3.40 \mathrm{E}-01\end{array}$ & $\begin{array}{l}2.38 \mathrm{E}+00 \pm \\
1.10 \mathrm{E}+00 \mathrm{UJ}\end{array}$ & $\begin{array}{c}2.72 \mathrm{E}+00 \pm \\
6.08 \mathrm{E}-01\end{array}$ & $\begin{array}{c}2.74 \mathrm{E}+00 \pm \\
6.63 \mathrm{E}-01\end{array}$ \\
\hline Iodine-129 & $\mathrm{U}$ & $\mathrm{U}$ & $\mathrm{U}$ & $\mathrm{U}$ & $\mathrm{U}$ \\
\hline Radium-228 & NS & NS & NS & NS & NS \\
\hline Strontium-90 & $\mathrm{U}$ & $\mathrm{U}$ & $\mathrm{U}$ & $\mathrm{U}$ & $\mathrm{U}$ \\
\hline Technicium 99 & $\mathrm{U}$ & $\mathrm{U}$ & $\mathrm{U}$ & $\mathrm{U}$ & $\mathrm{U}$ \\
\hline Tritium & $\begin{array}{c}8.91 \mathrm{E}+02 \pm \\
1.53 \mathrm{E}+02\end{array}$ & $\begin{array}{c}9.71 \mathrm{E}+02 \pm \\
1.54 \mathrm{E}+02\end{array}$ & $\mathrm{U}$ & $\begin{array}{c}1.11 \mathrm{E}+03 \pm \\
1.35 \mathrm{E}+02\end{array}$ & $\begin{array}{c}1.12 \mathrm{E}+03 \pm \\
1.36 \mathrm{E}+02\end{array}$ \\
\hline
\end{tabular}


Table C-3. Baseline groundwater data for Well ICPP-MON-A-166.

\begin{tabular}{|c|c|c|c|c|c|}
\hline \multirow[b]{2}{*}{ Analyzed Constituents } & \multicolumn{5}{|c|}{ Sample Dates } \\
\hline & $\begin{array}{c}\text { November } \\
2000 \\
\end{array}$ & $\begin{array}{c}\text { January } \\
2001 \\
\end{array}$ & $\begin{array}{c}\text { February } \\
2001 \\
\end{array}$ & $\begin{array}{c}\text { March } \\
2001 \\
\end{array}$ & $\begin{array}{l}\text { April } \\
2001 \\
\end{array}$ \\
\hline Miscellaneous Inorganics & $(\mathrm{mg} / \mathrm{L})$ & $(\mathrm{mg} / \mathrm{L})$ & $(\mathrm{mg} / \mathrm{L})$ & $(\mathrm{mg} / \mathrm{L})$ & $(\mathrm{mg} / \mathrm{L})$ \\
\hline Alkalinity & NS & NS & NS & 144 & 152 \\
\hline Biochemical Oxygen Demand & NS & NS & NS & NS & $\mathrm{U}$ \\
\hline Bromide & NS & NS & NS & NS & $\mathrm{U}$ \\
\hline Chloride & NS & NS & NS & NS & 10.9 \\
\hline Chromium hexavalent & NS & NS & NS & NS & $0.041 \mathrm{EJ}$ \\
\hline Fecal Coliform (COL/DL) & NS & NS & NS & NS & None \\
\hline Total Coliform (COL/DL) & NS & NS & NS & NS & 19 \\
\hline Fluoride & NS & NS & NS & NS & $0.31 \mathrm{EJ}$ \\
\hline Nitrate & NS & NS & NS & NS & $0.12 \mathrm{E}$ \\
\hline Nitrite & NS & NS & NS & NS & $\mathrm{U}$ \\
\hline Nitrogen, Ammonia (as N) & NS & NS & NS & $\mathrm{U}$ & $\mathrm{U}$ \\
\hline Nitrogen, Nitrate-Nitrite (as N) & NS & NS & NS & 0.0998 & 0.276 \\
\hline Phosphate & NS & NS & NS & NS & $\mathrm{U}$ \\
\hline Phosphorous, Total (as P) & NS & NS & NS & 0.0350 & 0.0411 \\
\hline Sulfate & NS & NS & NS & NS & 16.2 \\
\hline Total Dissolved Solids & NS & NS & NS & 220 & 217 \\
\hline Total Kjeldahl Nitrogen & NS & NS & NS & $\mathrm{U}$ & 0.240 \\
\hline TAL Metals & $(\mathrm{mg} / \mathrm{L})$ & $(\mathrm{mg} / \mathrm{L})$ & $(\mathrm{mg} / \mathrm{L})$ & $(\mathrm{mg} / \mathrm{L})$ & $(\mathrm{mg} / \mathrm{L})$ \\
\hline Aluminum & NS & NS & NS & 0.401 & 0.270 \\
\hline Antimony & NS & NS & NS & $\mathrm{U}$ & $\mathrm{U}$ \\
\hline Arsenic & NS & NS & NS & $\mathrm{U}$ & $\mathrm{U}$ \\
\hline Barium & NS & NS & NS & $0.0539 \mathrm{~B}$ & $0.0509 \mathrm{~B}$ \\
\hline Beryllium & NS & NS & NS & $\mathrm{U}$ & U \\
\hline Boron & NS & NS & NS & $0.0213 \mathrm{~B}$ & $\mathrm{U}$ \\
\hline Cadmium & NS & NS & NS & $\mathrm{U}$ & $\mathrm{U}$ \\
\hline Calcium & NS & NS & NS & 37.8 & 36.5 \\
\hline Chromium & NS & NS & NS & 0.0125 & $0.0084 \mathrm{~B}$ \\
\hline Cobalt & NS & NS & NS & $\mathrm{U}$ & $\mathrm{U}$ \\
\hline Copper & NS & NS & NS & $\mathrm{U}$ & $\mathrm{U}$ \\
\hline Iron & NS & NS & NS & 0.383 & 0.285 \\
\hline Lead & NS & NS & NS & $\mathrm{U}$ & 0.0044 \\
\hline Lithium & NS & NS & NS & $\mathrm{U}$ & $\mathrm{U}$ \\
\hline Magnesium & NS & NS & NS & 13.5 & 13.0 \\
\hline
\end{tabular}


Table C-3. (continued).

\begin{tabular}{|c|c|c|c|c|c|}
\hline \multirow[b]{2}{*}{ Analyzed Constituents } & \multicolumn{5}{|c|}{ Sample Dates } \\
\hline & $\begin{array}{c}\text { November } \\
2000\end{array}$ & $\begin{array}{c}\text { January } \\
2001 \\
\end{array}$ & $\begin{array}{c}\text { February } \\
2001\end{array}$ & $\begin{array}{c}\text { March } \\
2001\end{array}$ & $\begin{array}{l}\text { April } \\
2001\end{array}$ \\
\hline TAL Metals & $(\mathrm{mg} / \mathrm{L})$ & $(\mathrm{mg} / \mathrm{L})$ & $(\mathrm{mg} / \mathrm{L})$ & $(\mathrm{mg} / \mathrm{L})$ & $(\mathrm{mg} / \mathrm{L})$ \\
\hline Manganese & NS & NS & NS & 0.265 & 0.168 \\
\hline Mercury & NS & NS & NS & $\mathrm{U}$ & $\mathrm{U}$ \\
\hline Nickel & NS & NS & NS & 0.0586 & 0.0084 \\
\hline Potassium & NS & NS & NS & $2.62 \mathrm{~B}$ & $2.54 \mathrm{~B}$ \\
\hline Selenium & NS & NS & NS & $\mathrm{U}$ & $\mathrm{U}$ \\
\hline Silicon & NS & NS & NS & 27.2 & $12.4 \mathrm{~J}$ \\
\hline Silver & NS & NS & NS & $\mathrm{U}$ & $\mathrm{U}$ \\
\hline Sodium & NS & NS & NS & 12.9 & 11.3 \\
\hline Strontium & NS & NS & NS & 0.328 & 0.216 \\
\hline Thallium & NS & NS & NS & $\mathrm{U}$ & $\mathrm{U}$ \\
\hline Vanadium & NS & NS & NS & $0.0135 \mathrm{~B}$ & $\mathrm{U}$ \\
\hline Zinc & NS & NS & NS & 0.0306 & $0.0108 \mathrm{~B}$ \\
\hline CLP-List Volatile Organic Compounds & $(\mathrm{mg} / \mathrm{L})$ & $(\mathrm{mg} / \mathrm{L})$ & $(\mathrm{mg} / \mathrm{L})$ & $(\mathrm{mg} / \mathrm{L})$ & $(\mathrm{mg} / \mathrm{L})$ \\
\hline 1,1,1-Trichloroethane & NS & NS & NS & $\mathrm{U}$ & $0.005 \mathrm{UJ}$ \\
\hline 1,1,2,2-Tetrachloroethane & NS & NS & NS & $\mathrm{U}$ & $0.005 \mathrm{UJ}$ \\
\hline 1,1,2-Trichloroethane & NS & NS & NS & $\mathrm{U}$ & $0.005 \mathrm{UJ}$ \\
\hline 1,1-Dichloroethane & NS & NS & NS & $\mathrm{U}$ & $0.005 \mathrm{UJ}$ \\
\hline 1,1-Dichloroethene & NS & NS & NS & $\mathrm{U}$ & $0.005 \mathrm{UJ}$ \\
\hline 1,2-Dichloroethane & NS & NS & NS & $\mathrm{U}$ & $0.005 \mathrm{UJ}$ \\
\hline 1,2-Dichloroethene (Total) & NS & NS & NS & $\mathrm{U}$ & $0.005 \mathrm{UJ}$ \\
\hline 1,2-Dichloropropane & NS & NS & NS & $\mathrm{U}$ & $0.005 \mathrm{UJ}$ \\
\hline 2-Butanone & NS & NS & NS & $\mathrm{U}$ & $0.005 \mathrm{UJ}$ \\
\hline 2-Hexanone & NS & NS & NS & $\mathrm{U}$ & $0.005 \mathrm{UJ}$ \\
\hline 4-Methyl-2-pentanone & NS & NS & NS & $\mathrm{U}$ & $0.005 \mathrm{UJ}$ \\
\hline Acetone & NS & NS & NS & $\mathrm{U}$ & $0.005 \mathrm{UJ}$ \\
\hline Benzene & NS & NS & NS & $\mathrm{U}$ & $0.005 \mathrm{UJ}$ \\
\hline Bromodichloromethane & NS & NS & NS & $\mathrm{U}$ & $0.005 \mathrm{UJ}$ \\
\hline Bromoform & NS & NS & NS & $\mathrm{U}$ & $0.005 \mathrm{UJ}$ \\
\hline Bromomethane & NS & NS & NS & $\mathrm{U}$ & $0.005 \mathrm{UJ}$ \\
\hline Carbon disulfide & NS & NS & NS & $\mathrm{U}$ & $0.011 \mathrm{~J}$ \\
\hline Carbon tetrachloride & NS & NS & NS & $\mathrm{U}$ & $0.005 \mathrm{UJ}$ \\
\hline Chlorobenzene & NS & NS & NS & $\mathrm{U}$ & $0.005 \mathrm{UJ}$ \\
\hline Chloroethane & NS & NS & NS & $\mathrm{U}$ & $0.005 \mathrm{UJ}$ \\
\hline Chloroform & NS & NS & NS & $\mathrm{U}$ & $0.005 \mathrm{UJ}$ \\
\hline Chloromethane & NS & NS & NS & $\mathrm{U}$ & $0.005 \mathrm{UJ}$ \\
\hline
\end{tabular}


Table C-3. (continued).

\begin{tabular}{|c|c|c|c|c|c|}
\hline \multirow[b]{2}{*}{ Analyzed Constituents } & \multicolumn{5}{|c|}{ Sample Dates } \\
\hline & $\begin{array}{c}\text { November } \\
2000 \\
\end{array}$ & $\begin{array}{c}\text { January } \\
2001 \\
\end{array}$ & $\begin{array}{c}\text { February } \\
2001\end{array}$ & $\begin{array}{c}\text { March } \\
2001\end{array}$ & $\begin{array}{l}\text { April } \\
2001 \\
\end{array}$ \\
\hline CLP-List Volatile Organic Compounds & $(\mathrm{mg} / \mathrm{L})$ & $(\mathrm{mg} / \mathrm{L})$ & $(\mathrm{mg} / \mathrm{L})$ & $(\mathrm{mg} / \mathrm{L})$ & $(\mathrm{mg} / \mathrm{L})$ \\
\hline Dibromochloromethane & NS & NS & NS & $\mathrm{U}$ & $0.005 \mathrm{UJ}$ \\
\hline Ethylbenzene & NS & NS & NS & $\mathrm{U}$ & $0.005 \mathrm{UJ}$ \\
\hline Methylene Chloride & NS & NS & NS & $\mathrm{U}$ & $0.005 \mathrm{UJ}$ \\
\hline Styrene & NS & NS & NS & $\mathrm{U}$ & $0.005 \mathrm{UJ}$ \\
\hline Tetrachloroethene & NS & NS & NS & $\mathrm{U}$ & $0.005 \mathrm{UJ}$ \\
\hline Toluene & NS & NS & NS & 0.039 & $0.017 \mathrm{~J}$ \\
\hline Trichloroethene & NS & NS & NS & $\mathrm{U}$ & $0.005 \mathrm{UJ}$ \\
\hline Vinyl Chloride & NS & NS & NS & $\mathrm{U}$ & $0.005 \mathrm{UJ}$ \\
\hline Xylenes & NS & NS & NS & $\mathrm{U}$ & $0.005 \mathrm{UJ}$ \\
\hline Cis-1,3-Dichloropropene & NS & NS & NS & $\mathrm{U}$ & $0.005 \mathrm{UJ}$ \\
\hline Trans-1,3-Dichloropropene & NS & NS & NS & $\mathrm{U}$ & $0.005 \mathrm{UJ}$ \\
\hline Alpha emitters & $(\mathrm{pCi} / \mathrm{L})$ & $(\mathrm{pCi} / \mathrm{L})$ & $(\mathrm{pCi} / \mathrm{L})$ & $(\mathrm{pCi} / \mathrm{L})$ & $(\mathrm{pCi} / \mathrm{L})$ \\
\hline Americium 241 & NS & NS & NS & $\begin{array}{c}2.01 \mathrm{E}-02 \pm \\
8.05 \mathrm{E}-03 \mathrm{~J}\end{array}$ & $\begin{array}{c}2.14 \mathrm{E}-02 \pm \\
8.20 \mathrm{E}-03 \mathrm{~J}\end{array}$ \\
\hline Neptunium-237 & NS & NS & NS & $\mathrm{U}$ & $\mathrm{U}$ \\
\hline Plutonium 238 & NS & NS & NS & $\mathrm{U}$ & $\mathrm{U}$ \\
\hline Plutonium 239/240 & NS & NS & NS & $\mathrm{U}$ & $\mathrm{U}$ \\
\hline Uranium-233/234 & NS & NS & NS & $\begin{array}{c}1.16 \mathrm{E}+00 \pm \\
9.40 \mathrm{E}-02\end{array}$ & $\begin{array}{c}1.21 \mathrm{E}+00 \pm \\
1.30 \mathrm{E}-01\end{array}$ \\
\hline Uranium-235/236 & NS & NS & NS & $\begin{array}{c}4.58 \mathrm{E}-02 \pm \\
1.06 \mathrm{E}-02\end{array}$ & $\begin{array}{c}9.72 \mathrm{E}-02 \pm \\
2.69 \mathrm{E}-02\end{array}$ \\
\hline Uranium-238 & NS & NS & NS & $\begin{array}{c}5.26 \mathrm{E}-01 \pm \\
5.58 \mathrm{E}-02\end{array}$ & $\begin{array}{c}5.58 \mathrm{E}-01 \pm \\
7.42 \mathrm{E}-02\end{array}$ \\
\hline Gamma Emitters & $(\mathrm{pCi} / \mathrm{L})$ & $(\mathrm{pCi} / \mathrm{L})$ & $(\mathrm{pCi} / \mathrm{L})$ & $(\mathrm{pCi} / \mathrm{L})$ & $(\mathrm{pCi} / \mathrm{L})$ \\
\hline Americium 241 & NS & NS & NS & $\mathrm{U}$ & $\mathrm{U}$ \\
\hline Antimony-125 & NS & NS & NS & $\mathrm{U}$ & $\mathrm{U}$ \\
\hline Cerium-144 & NS & NS & NS & $\mathrm{U}$ & $\mathrm{U}$ \\
\hline Cesium-134 & NS & NS & NS & $\mathrm{U}$ & $\mathrm{U}$ \\
\hline Cesium-137 & NS & NS & NS & $\mathrm{U}$ & $\mathrm{U}$ \\
\hline Cobalt-58 & NS & NS & NS & $\mathrm{U}$ & $\mathrm{U}$ \\
\hline Cobalt- 60 & NS & NS & NS & $\mathrm{U}$ & $\mathrm{U}$ \\
\hline Europium-152 & NS & NS & NS & $\mathrm{U}$ & $\mathrm{U}$ \\
\hline Europium-154 & NS & NS & NS & $\mathrm{U}$ & $\mathrm{U}$ \\
\hline Europium-155 & NS & NS & NS & $\mathrm{U}$ & $\mathrm{U}$ \\
\hline Manganese-54 & NS & NS & NS & $\mathrm{U}$ & $\mathrm{U}$ \\
\hline Niobium-95 & NS & NS & NS & $\mathrm{U}$ & $\mathrm{U}$ \\
\hline
\end{tabular}


Table C-3. (continued).

\begin{tabular}{|c|c|c|c|c|c|}
\hline \multirow[b]{2}{*}{ Analyzed Constituents } & \multicolumn{5}{|c|}{ Sample Dates } \\
\hline & $\begin{array}{c}\text { November } \\
2000\end{array}$ & $\begin{array}{c}\text { January } \\
2001 \\
\end{array}$ & $\begin{array}{c}\text { February } \\
2001\end{array}$ & $\begin{array}{c}\text { March } \\
2001\end{array}$ & $\begin{array}{l}\text { April } \\
2001\end{array}$ \\
\hline Gamma Emitters & $(\mathrm{pCi} / \mathrm{L})$ & $(\mathrm{pCi} / \mathrm{L})$ & $(\mathrm{pCi} / \mathrm{L})$ & $(\mathrm{pCi} / \mathrm{L})$ & $(\mathrm{pCi} / \mathrm{L})$ \\
\hline Potassium-40 & NS & NS & NS & $\mathrm{U}$ & $\mathrm{U}$ \\
\hline Radium-226 & NS & NS & NS & $\begin{array}{c}4.54 \mathrm{E}+00 \pm \\
1.19 \mathrm{E}+00 \mathrm{~J}\end{array}$ & $\mathrm{U}$ \\
\hline Ruthenium-103 & NS & NS & NS & $\mathrm{U}$ & $\mathrm{U}$ \\
\hline Ruthenium-106 & NS & NS & NS & $\mathrm{U}$ & $\mathrm{U}$ \\
\hline Silver-108m & NS & NS & NS & $\mathrm{U}$ & $\mathrm{U}$ \\
\hline Silver-110m & NS & NS & NS & $\mathrm{U}$ & $\mathrm{U}$ \\
\hline Uranium-235 & NS & NS & NS & $\mathrm{U}$ & $\mathrm{U}$ \\
\hline Zinc-65 & NS & NS & NS & $\mathrm{U}$ & $\mathrm{U}$ \\
\hline Zirconium-95 & NS & NS & NS & $\mathrm{U}$ & $\mathrm{U}$ \\
\hline Isotopic Analyses & $(\mathrm{pCi} / \mathrm{L})$ & $(\mathrm{pCi} / \mathrm{L})$ & $(\mathrm{pCi} / \mathrm{L})$ & $(\mathrm{pCi} / \mathrm{L})$ & $(\mathrm{pCi} / \mathrm{L})$ \\
\hline Carbon-14 & NS & NS & NS & $\mathrm{U}$ & $\begin{array}{l}2.24 \mathrm{E}+00 \pm \\
9.14 \mathrm{E}-01 \mathrm{UJ}\end{array}$ \\
\hline Gross Alpha & NS & NS & NS & $\begin{array}{c}2.17 \mathrm{E}+00 \pm \\
6.03 \mathrm{E}-01\end{array}$ & $\begin{array}{c}1.12 \mathrm{E}+00 \pm \\
4.32 \mathrm{E}-01 \mathrm{~J}\end{array}$ \\
\hline Gross Beta & NS & NS & NS & $\begin{array}{c}2.94 \mathrm{E}+00 \pm \\
6.22 \mathrm{E}-01\end{array}$ & $\begin{array}{c}3.34 \mathrm{E}+00 \pm \\
5.80 \mathrm{E}-01\end{array}$ \\
\hline Iodine-129 & NS & NS & NS & $\mathrm{U}$ & $\mathrm{U}$ \\
\hline Radium-228 & NS & NS & NS & NS & $\mathrm{U}$ \\
\hline Strontium-90 & NS & NS & NS & $\mathrm{U}$ & $\mathrm{U}$ \\
\hline Technicium-99 & NS & NS & NS & $\mathrm{U}$ & $\mathrm{U}$ \\
\hline Tritium & NS & NS & NS & $\begin{array}{c}5.27 \mathrm{E}+02 \pm \\
1.30 \mathrm{E}+02\end{array}$ & $\mathrm{U}$ \\
\hline
\end{tabular}


Table C-4. Baseline groundwater data for Well ICPP-MON-A-167B.

\begin{tabular}{|c|c|c|c|c|c|c|}
\hline \multirow[b]{2}{*}{ Analyzed Constituents } & \multicolumn{6}{|c|}{ Sample Dates } \\
\hline & $\begin{array}{c}\text { November } \\
2000\end{array}$ & $\begin{array}{c}\text { January } \\
2001\end{array}$ & $\begin{array}{c}\text { February } \\
2001\end{array}$ & $\begin{array}{c}\text { March } \\
2001\end{array}$ & $\begin{array}{l}\text { April } \\
2001\end{array}$ & $\begin{array}{c}\text { April } \\
\text { Duplicate }\end{array}$ \\
\hline Miscellaneous Inorganics & $(\mathrm{mg} / \mathrm{L})$ & $(\mathrm{mg} / \mathrm{L})$ & $(\mathrm{mg} / \mathrm{L})$ & $(\mathrm{mg} / \mathrm{L})$ & $(\mathrm{mg} / \mathrm{L})$ & $(\mathrm{mg} / \mathrm{L})$ \\
\hline Alkalinity & 146 & 144 & 152 & 146 & 157 & 157 \\
\hline Biochemical Oxygen Demand & $8.33 \mathrm{BJ}$ & $6.15 B$ & $3.25 \mathrm{~B}$ & NS & $\mathrm{U}$ & $\mathrm{U}$ \\
\hline Bromide & NS & NS & NS & NS & $\mathrm{U}$ & $\mathrm{U}$ \\
\hline Chloride & NS & NS & 6.4 & NS & 9.6 & 9.5 \\
\hline Chromium hexavalent & NS & $\mathrm{R}$ & $\mathrm{U}$ & NS & $0.041 \mathrm{EJ}$ & $0.041 \mathrm{EJ}$ \\
\hline Fecal Coliform (COL/DL) & None & None & None & NS & None & None \\
\hline Total Coliform (COL/DL) & None & 1100 & 800 & NS & None & None \\
\hline Fluoride & NS & NS & 0.26 & NS & $0.19 \mathrm{EJ}$ & $0.24 \mathrm{EJ}$ \\
\hline Nitrate & 0.44 & 0.41 & 0.24 & NS & $0.53 \mathrm{E}$ & $0.52 \mathrm{E}$ \\
\hline Nitrite & $\mathrm{U}$ & $\mathrm{U}$ & $\mathrm{U}$ & NS & $\mathrm{U}$ & $\mathrm{U}$ \\
\hline Nitrogen, Ammonia (as N) & 0.0259 & 0.0332 & 0.0166 & 0.0792 & 0.0182 & $\mathrm{U}$ \\
\hline Nitrogen, Nitrate-Nitrite (as N) & 0.357 & 0.386 & 0.336 & 0.0826 & 0.449 & 0.501 \\
\hline Phosphate & NS & NS & $\mathrm{U}$ & NS & $\mathrm{U}$ & $\mathrm{U}$ \\
\hline Phosphorous, Total (as P) & 1.38 & 0.269 & 5.65 & 3.13 & 0.480 & 0.358 \\
\hline Sulfate & NS & NS & 30.0 & NS & 35.2 & 35.4 \\
\hline Total Dissolved Solids & 227 & 238 & 222 & 241 & 248 & 249 \\
\hline Total Kjeldahl Nitrogen & $\mathrm{U}$ & 0.141 & 0.143 & $0.705 \mathrm{~J}$ & 0.231 & 0.399 \\
\hline TAL Metals & $(\mathrm{mg} / \mathrm{L})$ & $(\mathrm{mg} / \mathrm{L})$ & $(\mathrm{mg} / \mathrm{L})$ & $(\mathrm{mg} / \mathrm{L})$ & $(\mathrm{mg} / \mathrm{L})$ & $(\mathrm{mg} / \mathrm{L})$ \\
\hline Aluminum & 32.8 & 27.2 & 17.7 & 23.7 & 15.6 & 14.2 \\
\hline Antimony & $\mathrm{U}$ & $\mathrm{U}$ & $\mathrm{U}$ & $\mathrm{U}$ & $\mathrm{U}$ & $\mathrm{U}$ \\
\hline Arsenic & $\mathrm{U}$ & $\mathrm{U}$ & $\mathrm{U}$ & $\mathrm{U}$ & $\mathrm{U}$ & $\mathrm{U}$ \\
\hline Barium & 0.113 & 0.103 & 0.0822 & $0.104 \mathrm{~B}$ & $0.0873 \mathrm{~B}$ & $0.0852 B$ \\
\hline Beryllium & $\mathrm{U}$ & $\mathrm{U}$ & $\mathrm{U}$ & $\mathrm{U}$ & $\mathrm{U}$ & $\mathrm{U}$ \\
\hline Boron & $\mathrm{U}$ & $\mathrm{U}$ & $\mathrm{U}$ & $\mathrm{U}$ & $\mathrm{U}$ & $\mathrm{U}$ \\
\hline Cadmium & $\mathrm{U}$ & $\mathrm{U}$ & $\mathrm{U}$ & $\mathrm{U}$ & $\mathrm{U}$ & $\mathrm{U}$ \\
\hline Calcium & 66.5 & 61.4 & 54.0 & 60.9 & 53.7 & 52.8 \\
\hline Chromium & 0.0438 & 0.045 & 0.0212 & 0.0325 & 0.0506 & 0.0481 \\
\hline Cobalt & 0.0152 & 0.0114 & 0.0064 & $0.0099 \mathrm{~B}$ & $0.0085 \mathrm{~B}$ & $0.0074 \mathrm{~B}$ \\
\hline Copper & 0.0706 & 0.0547 & 0.0349 & 0.0598 & 0.0334 & 0.031 \\
\hline Iron & 19.2 & 16.6 & 10.2 & 14.2 & 10.8 & 10.0 \\
\hline Lead & 0.0108 & 0.0076 & 0.0068 & 0.0086 & 0.0104 & 0.0095 \\
\hline Lithium & $\mathrm{U}$ & $\mathrm{U}$ & $\mathrm{U}$ & $\mathrm{U}$ & $\mathrm{U}$ & $\mathrm{U}$ \\
\hline Magnesium & 23.1 & 21.6 & 18.0 & 20.7 & 18.1 & 17.6 \\
\hline Manganese & 0.355 & 0.3 & 0.218 & 0.265 & 0.17 & 0.159 \\
\hline Mercury & $\mathrm{U}$ & $\mathrm{U}$ & $\mathrm{U}$ & $\mathrm{U}$ & $\mathrm{U}$ & $\mathrm{U}$ \\
\hline Nickel & 0.0801 & 0.0746 & 0.0438 & 0.0586 & 0.0549 & 0.0507 \\
\hline TAL Metals & $(\mathrm{mg} / \mathrm{L})$ & $(\mathrm{mg} / \mathrm{L})$ & $(\mathrm{mg} / \mathrm{L})$ & $(\mathrm{mg} / \mathrm{L})$ & $(\mathrm{mg} / \mathrm{L})$ & $(\mathrm{mg} / \mathrm{L})$ \\
\hline
\end{tabular}


Table C-4. (continued).

\begin{tabular}{|c|c|c|c|c|c|c|}
\hline \multirow[b]{2}{*}{ Analyzed Constituents } & \multicolumn{6}{|c|}{ Sample Dates } \\
\hline & $\begin{array}{c}\text { November } \\
2000\end{array}$ & $\begin{array}{c}\text { January } \\
2001\end{array}$ & $\begin{array}{l}\text { February } \\
2001\end{array}$ & $\begin{array}{l}\text { March } \\
2001\end{array}$ & $\begin{array}{l}\text { April } \\
2001\end{array}$ & $\begin{array}{c}\text { April } \\
\text { Duplicate }\end{array}$ \\
\hline Potassium & $2.56 \mathrm{~B}$ & $2.62 \mathrm{~B}$ & $2.3 \mathrm{~B}$ & $2.62 \mathrm{~B}$ & $2.42 \mathrm{~B}$ & $2.26 \mathrm{~B}$ \\
\hline Selenium & $\mathrm{U}$ & 0.0059 & $\mathrm{U}$ & $\mathrm{U}$ & $\mathrm{U}$ & $\mathrm{U}$ \\
\hline Silicon & 19.3 & 8.4 & 24.6 & 27.2 & 35.5 & 34.9 \\
\hline Silver & $\mathrm{U}$ & $\mathrm{U}$ & $\mathrm{U}$ & $\mathrm{U}$ & $\mathrm{U}$ & $\mathrm{U}$ \\
\hline Sodium & 14.0 & 13.3 & 12.0 & 12.9 & 15.4 & 15.1 \\
\hline Strontium & 0.349 & 0.323 & 0.294 & 0.328 & 0.296 & 0.292 \\
\hline Thallium & $\mathrm{U}$ & $\mathrm{U}$ & $\mathrm{U}$ & $\mathrm{U}$ & $\mathrm{U}$ & $\mathrm{U}$ \\
\hline Vanadium & 0.0207 & 0.0194 & 0.0124 & $0.0135 \mathrm{~B}$ & $0.0129 \mathrm{~B}$ & $0.0123 \mathrm{~B}$ \\
\hline Zinc & 0.0412 & 0.0337 & 0.0242 & 0.0306 & $0.0195 \mathrm{~B}$ & $0.0185 \mathrm{~B}$ \\
\hline $\begin{array}{l}\text { CLP-List Volatile Organic } \\
\text { Compounds }\end{array}$ & $(\mathrm{mg} / \mathrm{L})$ & $(\mathrm{mg} / \mathrm{L})$ & $(\mathrm{mg} / \mathrm{L})$ & $(\mathrm{mg} / \mathrm{L})$ & $(\mathrm{mg} / \mathrm{L})$ & $(\mathrm{mg} / \mathrm{L})$ \\
\hline 1,1,1-Trichloroethane & $\mathrm{U}$ & $\mathrm{U}$ & $\mathrm{U}$ & $\mathrm{U}$ & $\mathrm{U}$ & $\mathrm{U}$ \\
\hline 1,1,2,2-Tetrachloroethane & $\mathrm{U}$ & $\mathrm{U}$ & $\mathrm{U}$ & $\mathrm{U}$ & $\mathrm{U}$ & $\mathrm{U}$ \\
\hline 1,1,2-Trichloroethane & $\mathrm{U}$ & $\mathrm{U}$ & $\mathrm{U}$ & $\mathrm{U}$ & $\mathrm{U}$ & $\mathrm{U}$ \\
\hline 1,1-Dichloroethane & $\mathrm{U}$ & $\mathrm{U}$ & $\mathrm{U}$ & $\mathrm{U}$ & $\mathrm{U}$ & $\mathrm{U}$ \\
\hline 1,1-Dichloroethene & $\mathrm{U}$ & $\mathrm{U}$ & $\mathrm{U}$ & $\mathrm{U}$ & $\mathrm{U}$ & $\mathrm{U}$ \\
\hline 1,2-Dichloroethane & $\mathrm{U}$ & $\mathrm{U}$ & $\mathrm{U}$ & $\mathrm{U}$ & $\mathrm{U}$ & $\mathrm{U}$ \\
\hline 1,2-Dichloroethene (Total) & $\mathrm{U}$ & $\mathrm{U}$ & $\mathrm{U}$ & $\mathrm{U}$ & $\mathrm{U}$ & $\mathrm{U}$ \\
\hline 1,2-Dichloropropane & $\mathrm{U}$ & $\mathrm{U}$ & $\mathrm{U}$ & $\mathrm{U}$ & $\mathrm{U}$ & $\mathrm{U}$ \\
\hline 2-Butanone & $\mathrm{R}$ & $\mathrm{R}$ & $\mathrm{U}$ & $\mathrm{U}$ & $\mathrm{U}$ & $\mathrm{U}$ \\
\hline 2-Hexanone & $\mathrm{U}$ & $\mathrm{U}$ & $\mathrm{U}$ & $\mathrm{U}$ & $\mathrm{U}$ & $\mathrm{U}$ \\
\hline 4-Methyl-2-pentanone & $\mathrm{U}$ & $\mathrm{U}$ & $\mathrm{U}$ & $\mathrm{U}$ & $\mathrm{U}$ & $\mathrm{U}$ \\
\hline Acetone & $\mathrm{U}$ & $\mathrm{U}$ & $\mathrm{U}$ & $\mathrm{U}$ & $0.005 \mathrm{UJ}$ & $0.005 \mathrm{UJ}$ \\
\hline Benzene & $\mathrm{U}$ & $\mathrm{U}$ & $\mathrm{U}$ & $\mathrm{U}$ & $\mathrm{U}$ & $\mathrm{U}$ \\
\hline Bromodichloromethane & $\mathrm{U}$ & $\mathrm{U}$ & $\mathrm{U}$ & $\mathrm{U}$ & $\mathrm{U}$ & $\mathrm{U}$ \\
\hline Bromoform & $\mathrm{U}$ & $\mathrm{U}$ & $\mathrm{U}$ & $\mathrm{U}$ & $\mathrm{U}$ & $\mathrm{U}$ \\
\hline Bromomethane & $\mathrm{U}$ & $\mathrm{U}$ & $\mathrm{U}$ & $\mathrm{U}$ & $\mathrm{U}$ & $\mathrm{U}$ \\
\hline Carbon disulfide & $\mathrm{U}$ & $\mathrm{U}$ & $\mathrm{U}$ & $\mathrm{U}$ & $\mathrm{U}$ & $\mathrm{U}$ \\
\hline Carbon tetrachloride & $\mathrm{U}$ & $\mathrm{U}$ & $\mathrm{U}$ & $\mathrm{U}$ & $\mathrm{U}$ & $\mathrm{U}$ \\
\hline Chlorobenzene & $\mathrm{U}$ & $\mathrm{U}$ & $\mathrm{U}$ & $\mathrm{U}$ & $\mathrm{U}$ & $\mathrm{U}$ \\
\hline Chloroethane & $\mathrm{U}$ & $\mathrm{U}$ & $\mathrm{U}$ & $\mathrm{U}$ & $\mathrm{U}$ & $\mathrm{U}$ \\
\hline Chloroform & $\mathrm{U}$ & $\mathrm{U}$ & $\mathrm{U}$ & $\mathrm{U}$ & $\mathrm{U}$ & $\mathrm{U}$ \\
\hline Chloromethane & $\mathrm{U}$ & $\mathrm{U}$ & $\mathrm{U}$ & $\mathrm{U}$ & $\mathrm{U}$ & $\mathrm{U}$ \\
\hline Dibromochloromethane & $\mathrm{U}$ & $\mathrm{U}$ & $\mathrm{U}$ & $\mathrm{U}$ & $\mathrm{U}$ & $\mathrm{U}$ \\
\hline Ethylbenzene & $\mathrm{U}$ & $\mathrm{U}$ & $\mathrm{U}$ & $\mathrm{U}$ & $\mathrm{U}$ & $\mathrm{U}$ \\
\hline Methylene Chloride & $\mathrm{U}$ & $\mathrm{U}$ & $\mathrm{U}$ & $1 \mathrm{~J}$ & $0.005 \mathrm{UJ}$ & $0.005 \mathrm{UJ}$ \\
\hline Styrene & $\mathrm{U}$ & $\mathrm{U}$ & $\mathrm{U}$ & $\mathrm{U}$ & $\mathrm{U}$ & $\mathrm{U}$ \\
\hline Tetrachloroethene & $\mathrm{U}$ & $\mathrm{U}$ & $\mathrm{U}$ & $\mathrm{U}$ & $\mathrm{U}$ & $\mathrm{U}$ \\
\hline CLP-List Volatile Organic & $(\mathrm{mg} / \mathrm{L})$ & $(\mathrm{mg} / \mathrm{L})$ & $(\mathrm{mg} / \mathrm{L})$ & $(\mathrm{mg} / \mathrm{L})$ & $(\mathrm{mg} / \mathrm{L})$ & $(\mathrm{mg} / \mathrm{L})$ \\
\hline
\end{tabular}


Table C-4. (continued).

\begin{tabular}{|c|c|c|c|c|c|c|}
\hline \multirow[b]{2}{*}{ Analyzed Constituents } & \multicolumn{6}{|c|}{ Sample Dates } \\
\hline & $\begin{array}{c}\text { November } \\
2000\end{array}$ & $\begin{array}{c}\text { January } \\
2001\end{array}$ & $\begin{array}{c}\text { February } \\
2001\end{array}$ & $\begin{array}{l}\text { March } \\
2001\end{array}$ & $\begin{array}{l}\text { April } \\
2001\end{array}$ & $\begin{array}{c}\text { April } \\
\text { Duplicate }\end{array}$ \\
\hline \multicolumn{7}{|l|}{ Compounds } \\
\hline Toluene & $\mathrm{U}$ & $\mathrm{U}$ & $\mathrm{U}$ & $\mathrm{U}$ & $\mathrm{U}$ & $\mathrm{U}$ \\
\hline Trichloroethene & $\mathrm{U}$ & $\mathrm{U}$ & $\mathrm{U}$ & $\mathrm{U}$ & $\mathrm{U}$ & $\mathrm{U}$ \\
\hline Vinyl Chloride & $\mathrm{U}$ & $\mathrm{U}$ & $\mathrm{U}$ & $\mathrm{U}$ & $\mathrm{U}$ & $\mathrm{U}$ \\
\hline Xylenes & $\mathrm{U}$ & $\mathrm{U}$ & $\mathrm{U}$ & $\mathrm{U}$ & $\mathrm{U}$ & $\mathrm{U}$ \\
\hline Cis-1,3-Dichloropropene & $\mathrm{U}$ & $\mathrm{U}$ & $\mathrm{U}$ & $\mathrm{U}$ & $\mathrm{U}$ & $\mathrm{U}$ \\
\hline Trans-1,3-Dichloropropene & $\mathrm{U}$ & $\mathrm{U}$ & $\mathrm{U}$ & $\mathrm{U}$ & $\mathrm{U}$ & $\mathrm{U}$ \\
\hline Alpha emitters & $(\mathrm{pCi} / \mathrm{L})$ & $(\mathrm{pCi} / \mathrm{L})$ & $(\mathrm{pCi} / \mathrm{L})$ & $(\mathrm{pCi} / \mathrm{L})$ & $(\mathrm{pCi} / \mathrm{L})$ & $(\mathrm{pCi} / \mathrm{L})$ \\
\hline Americium-241 & $\mathrm{U}$ & $\mathrm{U}$ & $\begin{array}{l}1.34 \mathrm{E}-02 \pm \\
6.07 \mathrm{E}-03 \mathrm{~J}\end{array}$ & $\begin{array}{l}1.47 \mathrm{E}-02 \pm \\
6.35 \mathrm{E}-03 \mathrm{UJ}\end{array}$ & $\begin{array}{l}1.12 \mathrm{E}-01 \pm \\
3.29 \mathrm{E}-02\end{array}$ & $\begin{array}{c}3.89 \mathrm{E}-02 \pm \\
1.28 \mathrm{E}-02\end{array}$ \\
\hline Neptunium-237 & $\mathrm{U}$ & $\mathrm{U}$ & $\mathrm{U}$ & $\mathrm{U}$ & $\mathrm{U}$ & $\mathrm{U}$ \\
\hline Plutonium-238 & $\mathrm{U}$ & $\mathrm{U}$ & $\mathrm{U}$ & $\mathrm{U}$ & $\mathrm{U}$ & $\mathrm{U}$ \\
\hline Plutonium-239/240 & $\mathrm{U}$ & $\mathrm{U}$ & $\mathrm{U}$ & $\mathrm{U}$ & $\mathrm{U}$ & $\mathrm{U}$ \\
\hline Uranium-233/234 & $\begin{array}{c}1.46 \mathrm{E}+00 \pm \\
1.82 \mathrm{E}-01\end{array}$ & $\begin{array}{c}1.66 \mathrm{E}+00 \pm \\
1.62 \mathrm{E}-01\end{array}$ & $\begin{array}{c}1.32 \mathrm{E}+00 \pm \\
1.97 \mathrm{E}-01\end{array}$ & $\begin{array}{c}1.17 \mathrm{E}+00 \pm \\
1.16 \mathrm{E}-01\end{array}$ & $\begin{array}{c}1.37 \mathrm{E}+00 \pm \\
1.50 \mathrm{E}-01\end{array}$ & $\begin{array}{c}1.29 \mathrm{E}+00 \pm \\
1.37 \mathrm{E}-01\end{array}$ \\
\hline Uranium-235/236 & $\mathrm{U}$ & $\begin{array}{l}3.20 \mathrm{E}-02 \pm \\
1.46 \mathrm{E}-02 \mathrm{UJ}\end{array}$ & $\mathrm{U}$ & $\begin{array}{c}5.61 \mathrm{E}-02 \pm \\
1.73 \mathrm{E}-02\end{array}$ & $\begin{array}{c}3.76 \mathrm{E}-02 \pm \\
1.71 \mathrm{E}-02 \mathrm{~J}\end{array}$ & $\mathrm{U}$ \\
\hline Uranium-238 & $\begin{array}{c}7.65 \mathrm{E}-01 \pm \\
1.15 \mathrm{E}-01 \\
\end{array}$ & $\begin{array}{c}8.04 \mathrm{E}-01 \pm \\
9.25 \mathrm{E}-02 \\
\end{array}$ & $\begin{array}{c}9.82 \mathrm{E}-01 \pm \\
1.62 \mathrm{E}-01 \\
\end{array}$ & $\begin{array}{c}6.06 \mathrm{E}-01 \pm \\
7.10 \mathrm{E}-02 \\
\end{array}$ & $\begin{array}{c}6.62 \mathrm{E}-01 \pm \\
8.88 \mathrm{E}-02 \\
\end{array}$ & $\begin{array}{c}6.79 \mathrm{E}-01 \pm \\
8.59 \mathrm{E}-02 \\
\end{array}$ \\
\hline Gamma Emitters & $(\mathrm{pCi} / \mathrm{L})$ & $(\mathrm{pCi} / \mathrm{L})$ & $(\mathrm{pCi} / \mathrm{L})$ & $(\mathrm{pCi} / \mathrm{L})$ & $(\mathrm{pCi} / \mathrm{L})$ & $(\mathrm{pCi} / \mathrm{L})$ \\
\hline Americium-241 & $\mathrm{U}$ & $\mathrm{U}$ & $\mathrm{U}$ & $\mathrm{U}$ & $\mathrm{U}$ & $\mathrm{U}$ \\
\hline Antimony-125 & $\mathrm{U}$ & $\mathrm{U}$ & $\mathrm{U}$ & $\mathrm{U}$ & $\mathrm{U}$ & $\mathrm{U}$ \\
\hline Cerium-144 & $\mathrm{U}$ & $\mathrm{U}$ & $\mathrm{U}$ & $\mathrm{U}$ & $\mathrm{U}$ & $\mathrm{U}$ \\
\hline Cesium-134 & $\mathrm{U}$ & $\mathrm{U}$ & $\mathrm{U}$ & $\mathrm{U}$ & $\mathrm{U}$ & $\mathrm{U}$ \\
\hline Cesium-137 & $\mathrm{U}$ & $\mathrm{U}$ & $\mathrm{U}$ & $\mathrm{U}$ & $\mathrm{U}$ & $\mathrm{U}$ \\
\hline Cobalt-58 & $\mathrm{U}$ & $\mathrm{U}$ & $\mathrm{U}$ & $\mathrm{U}$ & $\mathrm{U}$ & $\mathrm{U}$ \\
\hline Cobalt-60 & $\mathrm{U}$ & $\mathrm{U}$ & $\mathrm{U}$ & $\mathrm{U}$ & $\mathrm{U}$ & $\mathrm{U}$ \\
\hline Europium-152 & $\mathrm{U}$ & $\mathrm{U}$ & $\mathrm{U}$ & $\mathrm{U}$ & $\mathrm{U}$ & $\mathrm{U}$ \\
\hline Europium-154 & $\mathrm{U}$ & $\mathrm{U}$ & $\mathrm{U}$ & $\mathrm{U}$ & $\mathrm{U}$ & $\mathrm{U}$ \\
\hline Europium-155 & $\mathrm{U}$ & $\mathrm{U}$ & $\mathrm{U}$ & $\mathrm{U}$ & $\mathrm{U}$ & $\mathrm{U}$ \\
\hline Manganese-54 & $\mathrm{U}$ & $\mathrm{U}$ & $\mathrm{U}$ & $\mathrm{U}$ & $\mathrm{U}$ & $\mathrm{U}$ \\
\hline Niobium-95 & $\mathrm{U}$ & $\mathrm{U}$ & $\mathrm{U}$ & $\mathrm{U}$ & $\mathrm{U}$ & $\mathrm{U}$ \\
\hline Potassium-40 & $\mathrm{U}$ & $\mathrm{U}$ & $\mathrm{U}$ & $\mathrm{U}$ & $\mathrm{U}$ & $\mathrm{U}$ \\
\hline Radium-226 & $\mathrm{U}$ & $\begin{array}{l}5.74 \mathrm{E}+00 \pm \\
1.69 \mathrm{E}+00 \mathrm{UJ}\end{array}$ & $\mathrm{U}$ & $\begin{array}{c}4.00 \mathrm{E}+00 \pm \\
1.16 \mathrm{E}+00\end{array}$ & $\mathrm{U}$ & $\mathrm{U}$ \\
\hline Ruthenium-103 & $\mathrm{U}$ & $\mathrm{U}$ & $\mathrm{U}$ & $\mathrm{U}$ & $\mathrm{U}$ & $\mathrm{U}$ \\
\hline Ruthenium-106 & $\mathrm{U}$ & $\mathrm{U}$ & $\mathrm{U}$ & $\mathrm{U}$ & $\mathrm{U}$ & $\mathrm{U}$ \\
\hline Silver-108m & $\begin{array}{c}1.18 \mathrm{E}+00 \pm \\
4.38 \mathrm{E}-01 \mathrm{UJ}\end{array}$ & $\mathrm{U}$ & $\mathrm{U}$ & $\mathrm{U}$ & $\mathrm{U}$ & $\mathrm{U}$ \\
\hline Silver-110m & $\mathrm{U}$ & $\mathrm{U}$ & $\mathrm{U}$ & $\mathrm{U}$ & $\mathrm{U}$ & $\mathrm{U}$ \\
\hline Gamma Emitters & $(\mathrm{pCi} / \mathrm{L})$ & $(\mathrm{pCi} / \mathrm{L})$ & $(\mathrm{pCi} / \mathrm{L})$ & $(\mathrm{pCi} / \mathrm{L})$ & $(\mathrm{pCi} / \mathrm{L})$ & $(\mathrm{pCi} / \mathrm{L})$ \\
\hline
\end{tabular}


Table C-4. (continued).

\begin{tabular}{|c|c|c|c|c|c|c|}
\hline \multirow[b]{2}{*}{ Analyzed Constituents } & \multicolumn{6}{|c|}{ Sample Dates } \\
\hline & $\begin{array}{c}\text { November } \\
2000\end{array}$ & $\begin{array}{c}\text { January } \\
2001\end{array}$ & $\begin{array}{c}\text { February } \\
2001\end{array}$ & $\begin{array}{l}\text { March } \\
2001\end{array}$ & $\begin{array}{l}\text { April } \\
2001\end{array}$ & $\begin{array}{c}\text { April } \\
\text { Duplicate }\end{array}$ \\
\hline Uranium-235 & $\mathrm{U}$ & $\mathrm{U}$ & $\mathrm{U}$ & $\mathrm{U}$ & $\mathrm{U}$ & $\mathrm{U}$ \\
\hline Zinc-65 & $\mathrm{U}$ & $\mathrm{U}$ & $\mathrm{U}$ & $\mathrm{U}$ & $\mathrm{U}$ & $\mathrm{U}$ \\
\hline Zirconium-95 & $\mathrm{U}$ & $\mathrm{U}$ & $\mathrm{U}$ & $\mathrm{U}$ & $\mathrm{U}$ & $\mathrm{U}$ \\
\hline Isotopic Analyses & $(\mathrm{pCi} / \mathrm{L})$ & $(\mathrm{pCi} / \mathrm{L})$ & $(\mathrm{pCi} / \mathrm{L})$ & $(\mathrm{pCi} / \mathrm{L})$ & $(\mathrm{pCi} / \mathrm{L})$ & $(\mathrm{pCi} / \mathrm{L})$ \\
\hline Carbon-14 & $\begin{array}{l}2.94 \mathrm{E}+00 \pm \\
7.87 \mathrm{E}-01 \mathrm{UJ}\end{array}$ & $\begin{array}{l}1.65 \mathrm{E}+00 \pm \\
7.22 \mathrm{E}-01 \mathrm{UJ}\end{array}$ & $\mathrm{U}$ & $\begin{array}{l}\text { 2.37E+00 } \pm \\
\text { 9.23E-01UJ }\end{array}$ & $\begin{array}{c}7.16 \mathrm{E}+00 \pm \\
9.65 \mathrm{E}-01\end{array}$ & $\begin{array}{c}2.86 \mathrm{E}+00 \pm \\
9.09 \mathrm{E}-01\end{array}$ \\
\hline Gross Alpha & $\begin{array}{c}5.09 \mathrm{E}+00 \pm \\
1.53 \mathrm{E}+00\end{array}$ & $\begin{array}{c}2.42 \mathrm{E}+00 \pm \\
7.02 \mathrm{E}-01\end{array}$ & $\begin{array}{c}6.00 \mathrm{E}+00 \pm \\
1.62 \mathrm{E}+00\end{array}$ & $\begin{array}{c}4.33 \mathrm{E}+00 \pm \\
1.51 \mathrm{E}+00 \mathrm{~J}\end{array}$ & $\begin{array}{l}2.12 \mathrm{E}+00 \pm \\
8.99 \mathrm{E}-01 \mathrm{UJ}\end{array}$ & $\begin{array}{l}9.54 \mathrm{E}-01 \pm \\
4.61 \mathrm{E}-01 \mathrm{UJ}\end{array}$ \\
\hline Gross Beta & $\begin{array}{c}9.80 \mathrm{E}+00 \pm \\
1.87 \mathrm{E}+00\end{array}$ & $\begin{array}{c}3.46 \mathrm{E}+00 \pm \\
6.72 \mathrm{E}-01\end{array}$ & $\begin{array}{c}3.45 \mathrm{E}+00 \pm \\
1.14 \mathrm{E}+00\end{array}$ & $\begin{array}{c}1.31 \mathrm{E}+01 \pm \\
1.90 \mathrm{E}+00\end{array}$ & $\begin{array}{c}3.38 \mathrm{E}+00 \pm \\
9.94 \mathrm{E}-01\end{array}$ & $\begin{array}{c}3.33 \mathrm{E}+00 \pm \\
5.33 \mathrm{E}-01\end{array}$ \\
\hline Iodine-129 & $\mathrm{U}$ & $\mathrm{U}$ & $\mathrm{U}$ & $\mathrm{U}$ & $\mathrm{U}$ & $\begin{array}{l}1.32 \mathrm{E}+00 \pm \\
4.64 \mathrm{E}-01 \mathrm{UJ}\end{array}$ \\
\hline Radium-228 & NS & NS & NS & NS & $\mathrm{U}$ & $\mathrm{U}$ \\
\hline Strontium-90 & $\mathrm{U}$ & $\mathrm{U}$ & $\mathrm{U}$ & $\mathrm{U}$ & $\mathrm{U}$ & $\mathrm{U}$ \\
\hline Technicium-99 & $\begin{array}{l}4.09 \mathrm{E}+00 \pm \\
1.89 \mathrm{E}+00 \mathrm{UJ}\end{array}$ & $\mathrm{U}$ & $\mathrm{U}$ & $\mathrm{U}$ & $\mathrm{U}$ & $\mathrm{U}$ \\
\hline Tritium & $\begin{array}{c}6.46 \mathrm{E}+02 \pm \\
1.09 \mathrm{E}+02\end{array}$ & $\begin{array}{c}5.99 \mathrm{E}+02 \pm \\
1.35 \mathrm{E}+02\end{array}$ & $\begin{array}{c}5.76 \mathrm{E}+02 \pm \\
1.17 \mathrm{E}+02 \\
\end{array}$ & $\begin{array}{c}3.61 \mathrm{E}+02 \pm \\
1.08 \mathrm{E}+02\end{array}$ & $\begin{array}{c}9.57 \mathrm{E}+02 \pm \\
1.23 \mathrm{E}+02\end{array}$ & $\begin{array}{c}9.68 \mathrm{E}+02 \pm \\
1.22 \mathrm{E}+02\end{array}$ \\
\hline
\end{tabular}




\section{Appendix D}

\section{Wellhead Protection Plan}


D-2 


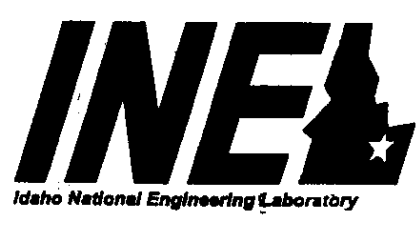

October 16. 1997

\section{LOCKHEED MATTINA}

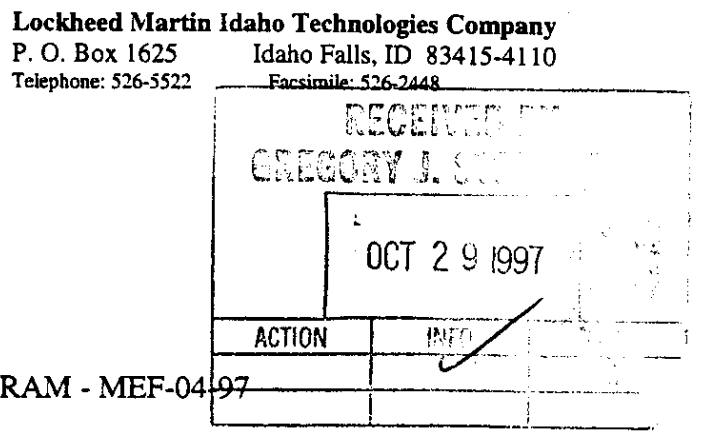

Distrabution

INEEL WELLHEAD PROTECTION PROGRAM - MEF-04

The Idaho Wellhead Protection Plan, published in February 1997 by the Idaho Division of Environmental Quality (DEQ), describes a program (currently voluntary) which recommends the establishment of a "Wellhead Protection Program" to prevent the contamination of drinking water wells This program is intended to protect drinking water supplies through the delineation of wellhead protection areas ${ }^{\mathrm{a}}$ followed by the implementation of management policies for these areas (and the potential contamination sources within them) relative to the levels of risk they pose. This Plan is soon to become regulation when adopted by EPA's Source Watèr Assessment program, but at the present time does not set implementation deadlines.

The Idaho National Engineering and Environmental Laboratory (INEEL) has chosen to begin implementation of a Wellhead Protection Program for all INEEL drinking-water and production wells prior to regulatorily enforced implementation deadlines. It is the intent at the INEEL to minimize impact to existing and future operations while at the same time establišhing aprogram which improves groundwater protection in cases where a significant risk to INEELiwater squrces now exists or may exist in the future. This can be accomplished by definining those areas in which contaminants (if released) could migrate to the drinking water and production wells, tising the appropriate INEEL organizations to evaluate the potential risks within these areas and establish appropriate controls and policies, and making INEEL personnel more cognizant of the areas of potential impact to INEEL water sources. In brief, it is intended to establish a program which requires that gromender and wellhead contamination risks be considered during INEEL ${ }^{2}$ operations' and projects; it is not the intent to discontinue or prohibit common INEEL activities within the Wellhead Protection Areas.

Implementation of a Wellhead Protection Prograan can be expected to create additional triancial. and regulatory responsibilities at the $\mathrm{NEEL} E$ In order to êstablish an effective Wellhead

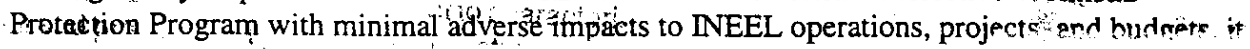

Wellhead protection areas are defined as those surface and subsurface areas surrounding a well through which contaminants are likely to move and contaminate the well over specified time periods. 
Distribution

October 16, 1997

MEF-04-97

Page 2

is important that the appropriate people be involved during program development. Attachment $A$ describes IDEQ's Wellhead Protection Plan requirements, discusses the NEEL activities conducted to date, and addresses future program implementation tasks, while Attachment B presents proposed wellhead protection area maps for the INEEL. Please review the Attachments (or pass them on to an appropriate responsible party) and provide comments back to me by November 15, 1997 regarding designated points of contact for further development of the INEEL's Wellhead Protection Program, specific interests or concerns you may have regarding this program, and the proposed wellhead protection area maps. I can be reached at 526-5522, Mailstop 4110, or OV address "FELDME".

Sincerely,

Mres recunte)

Mark Feldman

Environmental Monitoring

MEF:caq

Attachments 
Distribution

October 16, 1997

MEF-04-97

Page 3

Distribution

ANL-W

C. J. Martin, MS-6000

DOE-ID

C. M. Bennett, MS-1146

WEC

R. W. Nieslanik, MS-6001

K. D. Willie, MS-6001

\section{LMITCO}

E. M. Balsmeier, MS-7137

T. L. Carlson, MS-7137

M. J. Edwards, MS-9204

D. L. Forsberg, MS-4201

C. R. Hickman, MS-3530

J. R. Jansen, MS-8101

R. D. Johnson, MS-5117

R. K. Jones, MS-3921

J. C. Kvamme, MS-4201

R. M. Macfarlane, MS-4109 .

M. D. Sandvig, MS-0313

T. M. Stoops, MS-3953

D. G. Venable, MS-3427

M. Vorachek, MS-5208

E. C. Miller, LMITCO, MS-3953

R. A. Montgomery, LMITCO, MS-3427

B. R. Orr, USGS, MS -4148

G. Sehlke, LMITCO, MS-4110

G. J. Stormberg, LMITCO, MS-2107

1.4 V. Street, LMITCO, MS-4110

E. M. Walker, LMITCO, MS-4110

T. R. Wood, LMITCO, MS-3954

M. E. Feldman File 
Attachment A

The Idaho Wellhead Protection Plan has three general goals: to prevent the contamination of groundwater by appropriately managing potential contamination sources; to establish "response action areas" around a wellhead which provides sufficient time to respond to an event which could contaminate drinking water supplies; and to protect the area of contribution of a well by appropriately managing land use. The Plan attempts to accomplish these goals by requiring a number of actions, including delineating wellheàd protection areas for each drinking water well, maintaining an inventory and management strategy for the potential contamination sources within these areas, and developing land-use polkcies which protect the areas of contribution to a well. It also requires that contingency plans beteveloped in the event a drinking water well becomes contaminated, and that sites for future wells be identified and protected.

The Plan recognizes that the "management" and "policy" components are the most important parts of the Plan, and provides much latitude to the water purveyor in the manner in which most requirements can be met. The exception to this is the delineation of the wellhead protection areas; boundaries must be established using the guidelines presented. The Plan requires that four distinct zones be identified for each well. These zones, presented in order of the stringency with which they should be managed, include:

(1) Zone $1 \mathrm{~A}$, which corresponds to a 50 foot sanitary setback radius from the well (presently required by state law),

(2) Zone 1B, which corresponds to the boundary for a 3-year time of travel for contaminants in the aquifer,

(3) Zone 2, which corresponds to the boundary for a 6-year time of travel for contaminants in the aquifer, and

(4) Zone 3, which corresponds to the boundary for a 10-year time of travel for contaminants in the aquifer.

The Plan also identifies 5 methods for delineation, dependent upon the amount of information available, and cautions that any assumptions made on behalf of information uncertainties should be made such that the delineation analyses yield the largest (most protective) wellhead protection area.

The INEEL decided 2-3 years ago to begin implementation of the requirements of IDEQ'sWellhead Protection Plan as a "best management practice" for all drinking water and production wells because the Plan represented a sensible approach to groundwater protection. The ensuing activities in 1995 resulted in the preparation of "capture zone" maps for all the INEEL wells, but little else in the form of implementation. More recently, IDEQ submitted the updated Plan (published in February 1997) as a proposal to meet EPA's Source Water Assessment program requirements and, based on initial feedback from EPA expects approval of the Plan in its current form. This action is expected to make the requirements mandatory within the next 2 years, and has sparked renewed interest in the program. As an initial step, proposed wellhead protection area maps have been prepared for each of the INEEL facilities in which these wells are located. These maps were created by modifying the previously prepared capture zone maps to meet the current requirements of the IDEQ Plan, and include the four designated protection area zones. 
During preparation of the maps, efforts were made to balance the total area impacted against the need to identify all areas which may be in the zone of contribution for a wellhead. The result is a series of maps which represent conservative boundaries for wellhead protection areas based on conditions which have been documented over time at the INEEL. The boundaries for these wellhead protection areas do not imply that all areas encompassed actually contribute to the wellhead, but more accurately they suggest that contribution to the wellhead is possible within these areas. This is consistent with the intended use of the maps, which is to indicate those regions in which (1) added care should be given to those existing sites which represent potential groundwater contamination sources and (2) speciât consideration should be given to future construction which may represent a groundwater risk. (The maps cannot be used to definitively state that a site, specifically one in close proximity to a protection area boundary, is or is not in the catchment zone for a wellhead.) Attachment B.provides a detailed discussion of the delineation process for the INEEL's wellhead protection areas, and presents the maps and tabular information on the protection zones.

Future INEEL Wellhead Protection Program activities include finalizing the wellhead protection area maps and completing the inventory of potential contamination sources, determining programmatic responsibilities and implementation avenues, and developing appropriate policies for managing potential contamination sources and land use within the protection zone boundaries. The wellhead protection area maps can be finalized after incorporation of any feedback on the proposed delineations in Attachment B, and the initial inventory of potential contamination sources (which has already begun) can be based upon information already documented in the INEEL's GIS library and other readily available resources. Concurrent with this, discussions regarding the appropriate organizations for ownership of responsibilities should be initiated, with the intent to use those organizations and programs already performing similar tasks. (Examples include possible use of the Environmental Restoration organization to evaluate the relative risk of existing and future potential contamination sources within the wellhead protection areas; the INEEL Groundwater Protection Program Plan and the INEEL Land Use Planning Document to publicize the wellhead protection area maps and provide easy access for their use across the INEEL; and the National Environmental Policy Act project review checklist for early identification of $\mathrm{NEEL}$ operations and projects which fall within the protection areas.) Policy development efforts for the INEEL Wellhead Protection Program should begin soon thereafter. Based on recommendations of the INEEL Groundwater Committee, the focus of these policies should be to identify (and minimize where feasible) existing risks to INEEL water -sources while at the same time protecting these water sources from future NEEL activities through informed siting and controls. Emphasis should be placed on a "common sense" approach which avoids the practice of summarily prohibiting activities and projects within wellhead protection areas. 


\section{Attachment B}

The Idaho Wellhead Protection Plan identifies five techniques for the delineation of wellhead protection areas; three of these techniques (the Basic I, Basic II, and Refined Methods) can be applied at the INEEL. The Basic I and II Methods are both conservative techniques which establish four large, concentric, fixed-radius circles around each wellhead as boundaries for the four protection area zones, while the Refined Method allows the water purveyor to use knowledge of the aquifer properties to establish the protection area zones and boundaries. (The Refined Method estimates a capture zone boundary for a wellhead using various modelling codes and requires access to data such as transmissivity, hydraulic gradient, flow angle, and pump rate.) Given sufficient information, the Refined Method provides a better estimation of the actual catchment area for a wellhead, as well as a smaller protection area which must be managed.

The wellhead protection areas delineated for the INEEL drinking water and production wells are based upon the Refined Method. In 1995, the RESSQC Module of EPA's WHPA modelling code was selected for use from the list of modelling codes and simple "capture zone" maps were generated for 2 and 5-year times of travel for each of the wells. In 1997, when work on the NEEL's Wellhead Protection Program was reinitiated, the previous capture zone maps were compared to IDEQ's current Wellhead Protection Plan requirements. Changes and details highlighted in the Plan (basing protection area zones on 3,6, and 10-year times of travel, requiring the use of the most conservative assumptions when faced with data uncertainties or ranges of values, and noting that the WHPA code assumes no lateral migration of contaminants through the vadose zone) implied that the capture zone maps prepared in 1995 no longer satisfy Plan requirements. Faced with three options (1) adopt much larger protection areas via the use of one of the Basic Methods, (2) embark on an expensive mission to remodel the capture zones using a more complex code, or (3) modify the existing capture zone maps, the latter was chosen.

Based on the recommendations and assistance from the INEEL Groundwater Committee, the capture zone maps were modified in a number of ways. First, the protection areas were lengthened by linearly extrapolating the 2 and 5-year time of travel capture zones to 3,6, and 10year times of travel. Second, the protection areas were widened by adopting flow angle "ranges", rather than the single, dominant flow angle used in the original capture zone maps. Based on information in the 1993 "INEL Groundwater Monitoring Plan" (GMP) all INEEL facilitiesexhibit variation or uncertainty in aquifer flow angle ranging from 20 degrees to 90 degrees. (Identified and documented in the GMP for the purpose of siting monitoring wells at NEEL facilities, these ranges are believed to represent conservative values.) Adopting these flow angle ranges as bounding guidelines in establishing the lateral boundaries of the protection areas, the resultant protection areas resemble a pie-shaped wedge.

Third, the protection areas were augmented with a circular zone surrounding the wellhead to account for uncertainties associated with a heterogeneous aquifer, a thick vadose zone, and lateral migration of contaminants prior to their entry to the aquifer. The GMP, in the Monitoring Network sections for each facility, recognizes that ample opportunity exists for lateral migration of contaminants through the vadose zone due to its thickness and fractured basalt structure. As a 
result, it defines the distance the "line of compliance" monitoring wells should be located from the potential contamination sources in order to capture any lateral migration. In effect, these distances provide a site-specific estimate of the potential for lateral movement of the contaminants through the vadose zone. Ranging from 500 to 4000 feet, these values represent the best facility-specific information available, and were adopted as the radii for the circular management zones around the wellheads.

Finally, as recommended by IDEQ's plan, in situations where the mapped protection areas of multiple wells intersect, the protection areas are combined and the wells are treated as a single wellfield. This has resulted in a single wellhead,protection area for each of the INEEL facilities within which drinking water or production wells are located. Table 1 provides a listing of the wells by facility and summarizes the final speciffcations used in delineating the protection areas at each of the INEEL's facilities, and figures 1-18 present the maps showing the protection area zones and boundaries. As can be expected and is seen in the figures, most of the INEEL's wellhead protection areas are common in shape and orientation, though some abnormalities exist. Those figures which demonstrate abnormal characteristics are discussed below.

The INEEL and all of its wellhead protection areas are shown in Figure 1, providing an indication of the total areas covered. As expected, this figure shows variation in protection area size when comparing one facility to another. This is largely the result of the variation in transmissivity values (which dictates protection area length) and groundwater flow angle range values (which dictates protection area width) across the site. Close observation of this figure also points out an apparent discrepancy; NRF and ICPP portray similar wellhead protection area characteristics, but TRA has a protection area which differs significantly in size and orientation, even though it overlaps that of ICPP. This is probably caused by facility-specific data being extrapolated to a larger region. (Local hydrologic conditions vary greatly in fractured basalt; when these localized conditions are projected over a larger area, subtle differences in the data become magnified.) These differences do not necessarily indicate an error in the development of the protection area for TRA, but more likely reflect the degree of uncertainty associated with establishing the protection areas for NRF and ICPP. As a result, no effort to change the protection area maps to reflect "consistency" have been made.

The EBR-I and RWMC wellhead protection areas, shown in figures 6 and 13 respectively, each have circular protection areas rather than the characteristic wedge-shaped protection areas. - This is due to the low transmissivity values measured at each facility, resulting in relatively short distances modelled for 10-year times of travel (296 feet for EBR-I and 1800 feet for RWMC). In both cases, the distance associated with the "potential for lateral movement of contaminants through the vadose zone" exceeds the linear distance for the 10-year time of travel. As a result, the circular zone placed around the wellhead to account for lateral contaminant movement in the vadose zone is the dominant feature, and has been designated the protection area boundary for both maps. (Consistent with this boundary designation, the lines marking Zones $1 \mathrm{~B}$ and 2 were adjusted accordingly.) This technique is supported at RWMC by the fact that contaminants believed to have originated in the Subsurface Disposal Area (which is not within the flow angle range measured at the RWMC) have been detected at the wellhead. 
The ICPP wellhead protection area, shown in figures 8 and 9 , is notable simply for its size. Covering approximately 40 square miles and enveloping the NRF facility, this protection area is influenced by the high transmissivity values measured at ICPP and a wide aquifer flow angle range which is probably due to the influence of the Big Lost River. This protection area also has the largest circular zone surrounding the wellheads, owing to a history of significant contaminant movement along the perched water zones below ICPP. (Contaminants, believed to have originated south of the wells and well outside the groundwater flow angle range for ICPP, have been detected in the ICPP Production Wells $04 \&$ 05.) As noted previously, the boundaries for this wellhead protection area are based on the information specific to the localized conditions at ICPP. Projection of these conditions over a larger area to establish boundaries for the ICPP wellhead protection area has likely resulted in a very conservatively sized protection area, but is also reflective of the lack of information available on the area between ICPP and NRF.

The rest of the wellhead protection areas, as shown in the remainder of the figures, are very similar in nature and exhibit no notable abnormalities. 
Table 1 - INEEL Wellhead Protection Area Delineation Specifications

\begin{tabular}{|c|c|c|c|c|c|c|}
\hline $\begin{array}{l}\text { FACILITY; } \\
\text { Well ID }\end{array}$ & $\begin{array}{l}\text { Zone } 1 A^{*} \\
\text { Radius } \\
\text { (ft) }\end{array}$ & $\begin{array}{l}\text { Circular } \\
\text { Zone }^{\mathrm{b}} \text { Radius } \\
\text { (ft) }\end{array}$ & $\begin{array}{l}\text { Boundary } \\
\text { Angles }{ }^{c} \\
\text { (degrees) }\end{array}$ & $\begin{array}{l}\text { Zone } 1 B^{d} \\
\text { Length } \\
\text { (ft) }\end{array}$ & $\begin{array}{l}\text { Zone } 2^{e} \\
\text { Length } \\
\text { (ft) }\end{array}$ & $\begin{array}{l}\text { Zone } 3^{r} \\
\text { Length } \\
\text { (ft) }\end{array}$ \\
\hline $\begin{array}{l}\text { ANL-W ; } \\
\text { EBR-II \#1\&2 }\end{array}$ & 50 & 500 & $\begin{array}{l}20 \text { degree span from } \\
45-65 \text { degrees }\end{array}$ & 6200 & 12400 & 20000 \\
\hline $\begin{array}{l}\text { CFA; } \\
\text { CFA \#1\&2 }\end{array}$ & 50 & 1000 & $\begin{array}{l}35 \text { degree span from } \\
0-35 \text { degrees }\end{array}$ & 2833 & 5667 & 9000 \\
\hline $\begin{array}{l}\text { EBR-I; } \\
\text { EBR-I }\end{array}$ & 50 & 500 & $\begin{array}{l}360 \text { degree span } \\
\text { trom } 0-360 \text { degrees }\end{array}$ & 150 & 300 & 500 \\
\hline $\begin{array}{l}\text { Gun Range; } \\
\text { Rifle Range Well }\end{array}$ & 50 & 5008 & $\begin{array}{l}80 \text { degree span from } \\
0-80 \text { degrees }\end{array}$ & 3767 & 7533 & 12600 \\
\hline $\begin{array}{l}\text { ICPP; } \\
\text { CPP \#01, 02, } 04 \\
\& 05\end{array}$ & 50 & 4000 & $\begin{array}{l}90 \text { degree span from } \\
325 \text { - } 55 \text { degrees }\end{array}$ & 11533 & 23067 & 38000 \\
\hline $\begin{array}{l}\text { NRF; } \\
\quad \text { NRF \#1, 2, \& } 3\end{array}$ & 50 & $500^{8}$ & $\begin{array}{l}60 \text { degree span from } \\
330-30 \text { degrees }\end{array}$ & 6700 & 13400 & 22400 \\
\hline $\begin{array}{l}\text { PBF; } \\
\text { SPERT \#1 \& } 2\end{array}$ & 50 & 500 & $\begin{array}{l}30 \text { degree span from } \\
25 \text { - } 55 \text { degrees }\end{array}$ & 1087 & 2173 & 3600 \\
\hline $\begin{array}{l}\text { RWMC; } \\
\text { Production Well }\end{array}$ & 50 & 2000 & $\begin{array}{l}360 \text { degree span } \\
\text { from } 0-360 \text { degrees }\end{array}$ & 600 & 1200 & 2000 \\
\hline $\begin{array}{l}\text { TRA; } \\
\text { TRA \#01, 03, } \\
\text { \& } 04\end{array}$ & 50 & 1000 & $\begin{array}{l}30 \text { degree span from } \\
30-60 \text { degrees }\end{array}$ & 3067 & 6133 & 9200 \\
\hline $\begin{array}{l}\text { TAN-CTF; } \\
\text { FET \#1\& } 2\end{array}$ & 50 & 500 & $\begin{array}{l}55 \text { degree span from } \\
355 \text { - } 50 \text { degrees }\end{array}$ & 500 & 800 & 1200 \\
\hline $\begin{array}{l}\text { TAN-TSF; } \\
\text { ANP \#01 \& } 02\end{array}$ & 50 & 500 & $\begin{array}{l}90 \text { degree span from } \\
315 \text { - } 25 \text { degrees }\end{array}$ & 667 & 1333 & 2000 \\
\hline $\begin{array}{l}\text { TAN-WRRTF; } \\
\text { ANP } \# 08\end{array}$ & 50 & 500 & $\begin{array}{l}75 \text { degree span from } \\
310-25 \text { degrees }\end{array}$ & 500 & 1033 & 1700 \\
\hline
\end{tabular}

- Sanitary setback distance defined by the State of Idaho for drinking water wells.

b Circular protection zone for the lateral migration of contaminants within the vadose zone, based on information in the "INEL Groundwater Monitoring Plan".

c Lateral protection area boundaries measured clockwise from due North.

d Estimated distance for a 3-year time of travel in the aquifer.

- Estimated distance for a 6-year time of travel in the aquifer.

f Estimated distance for a 10-year time of travel in the aquifer.

- The "INEL Groundwater Monitoring Plan" does not identify a distance for these facilities -- 500' was selected as the minimum default value. 


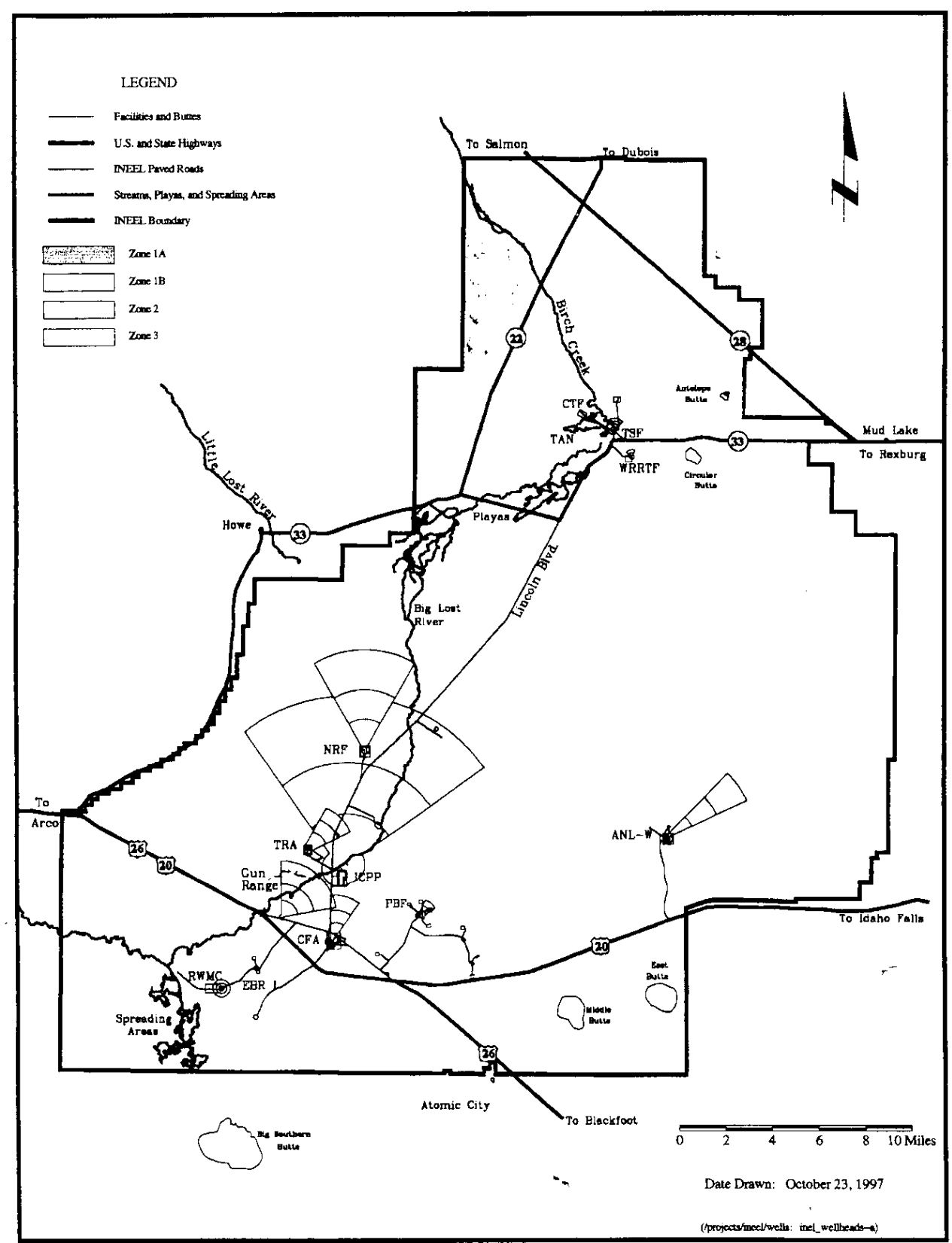

Flgure 1. INEEL Wellhead Protection Areas. 


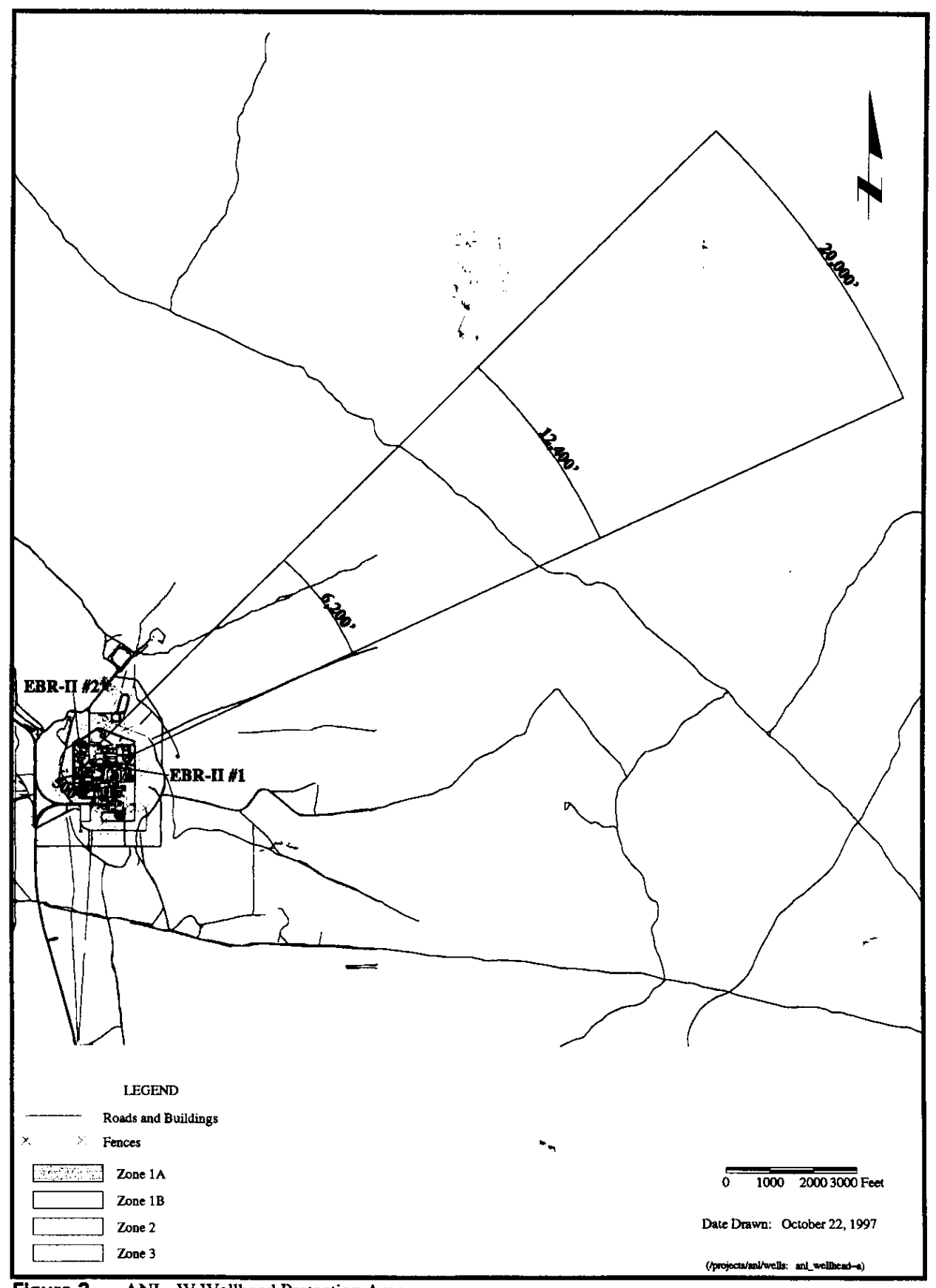

Figure 2. ANL-W Wellhead Protection Area. 


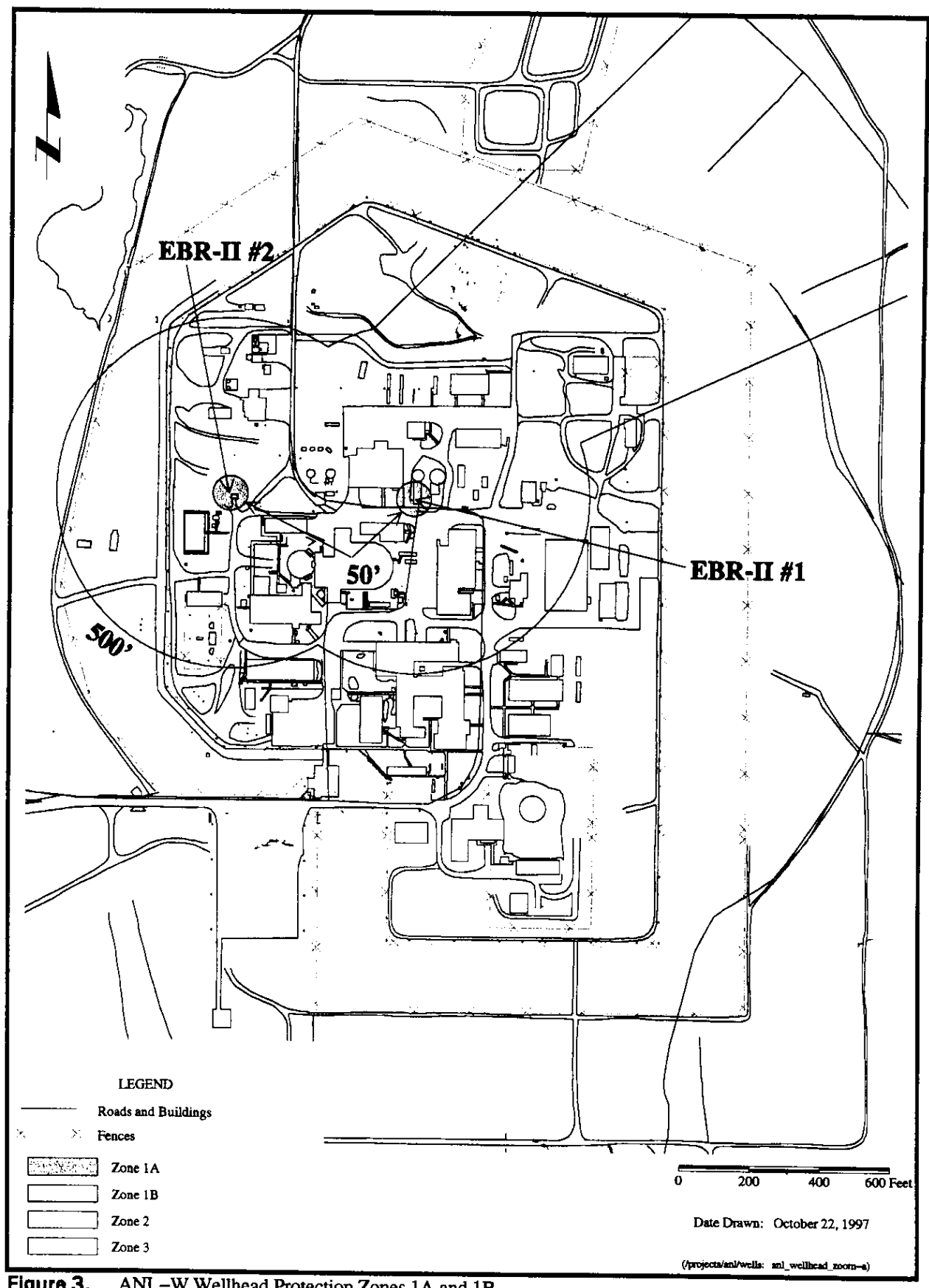

Figure 3. ANL-W Wellhead Protection Zones 1A and 1B. 


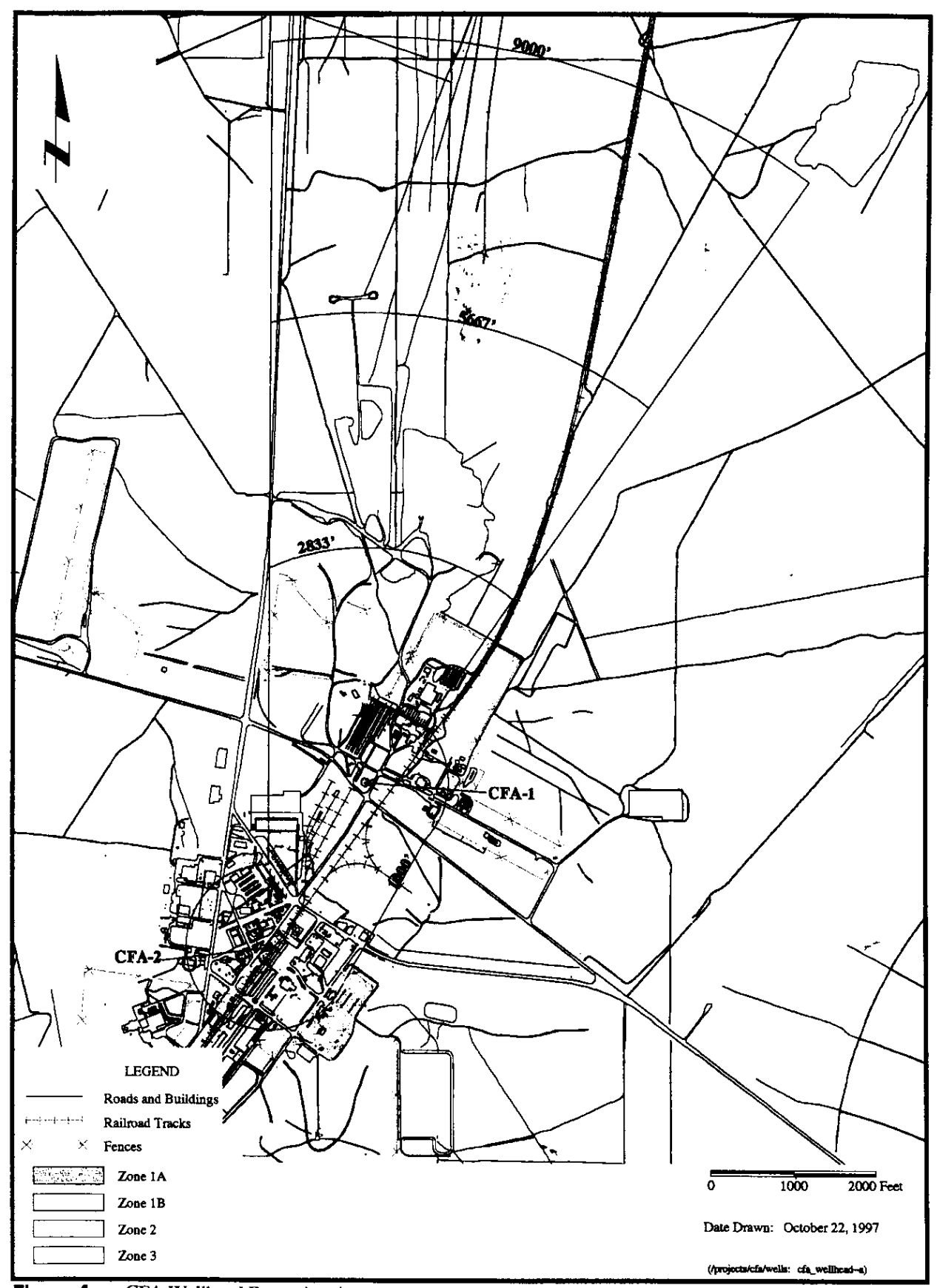

Figure 4. CFA Wellhead Protection Area. 


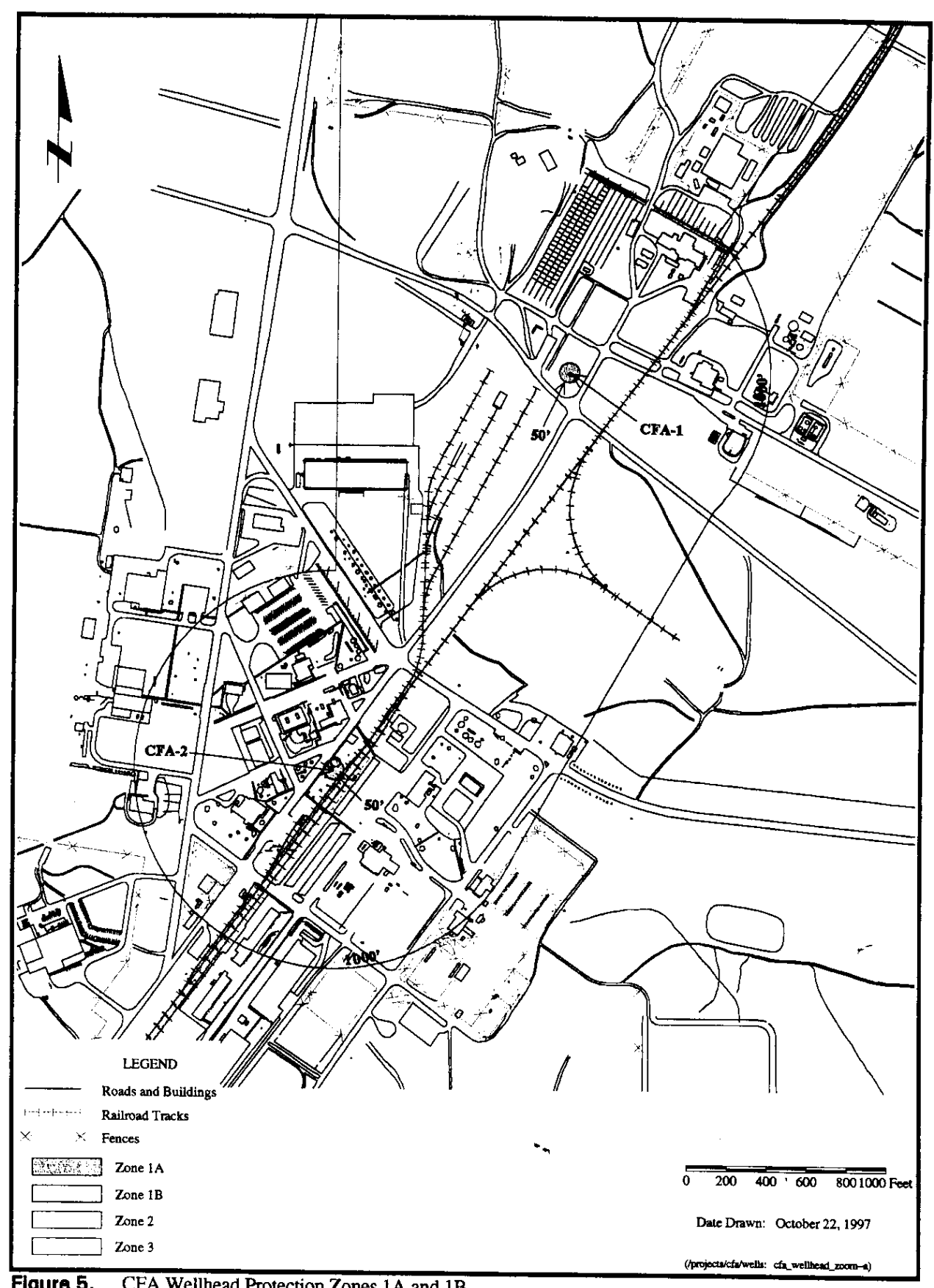

Figure 5. CFA Wellhead Protection Zones 1A and 1B. 


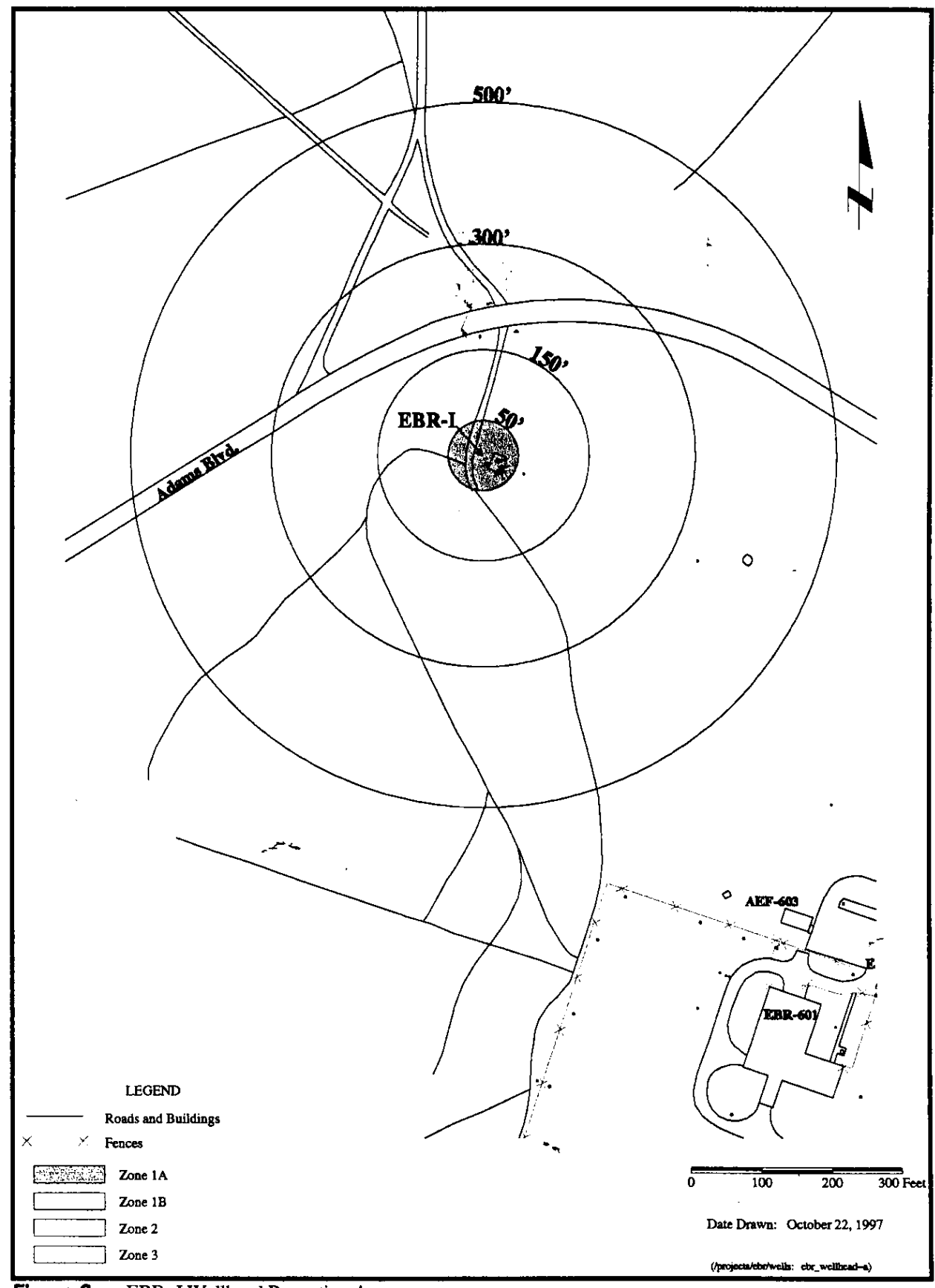

Figure 6. EBR-I Wellhead Protection Area. 


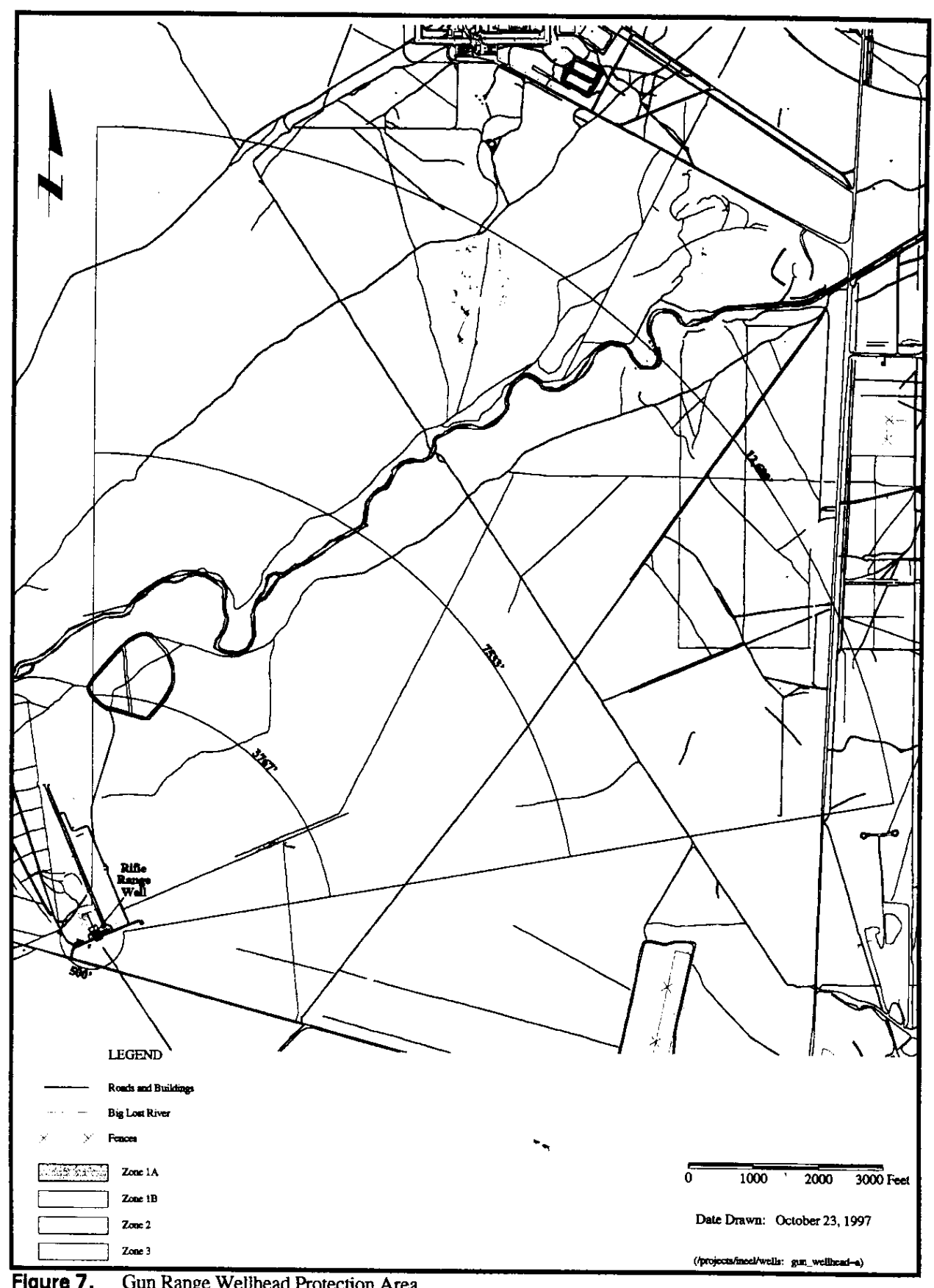




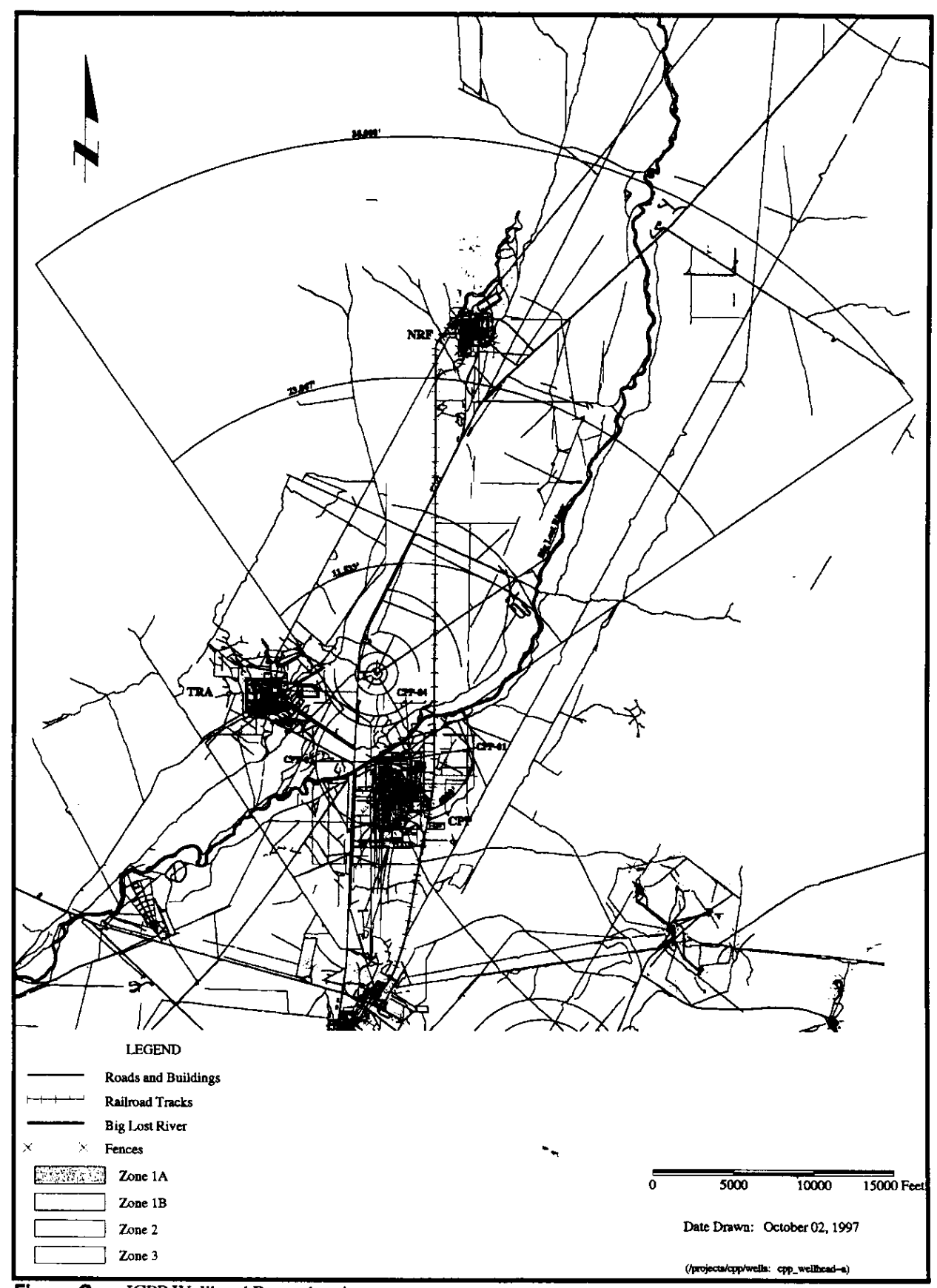

Figure 8. ICPP Wellhead Protection Area. 


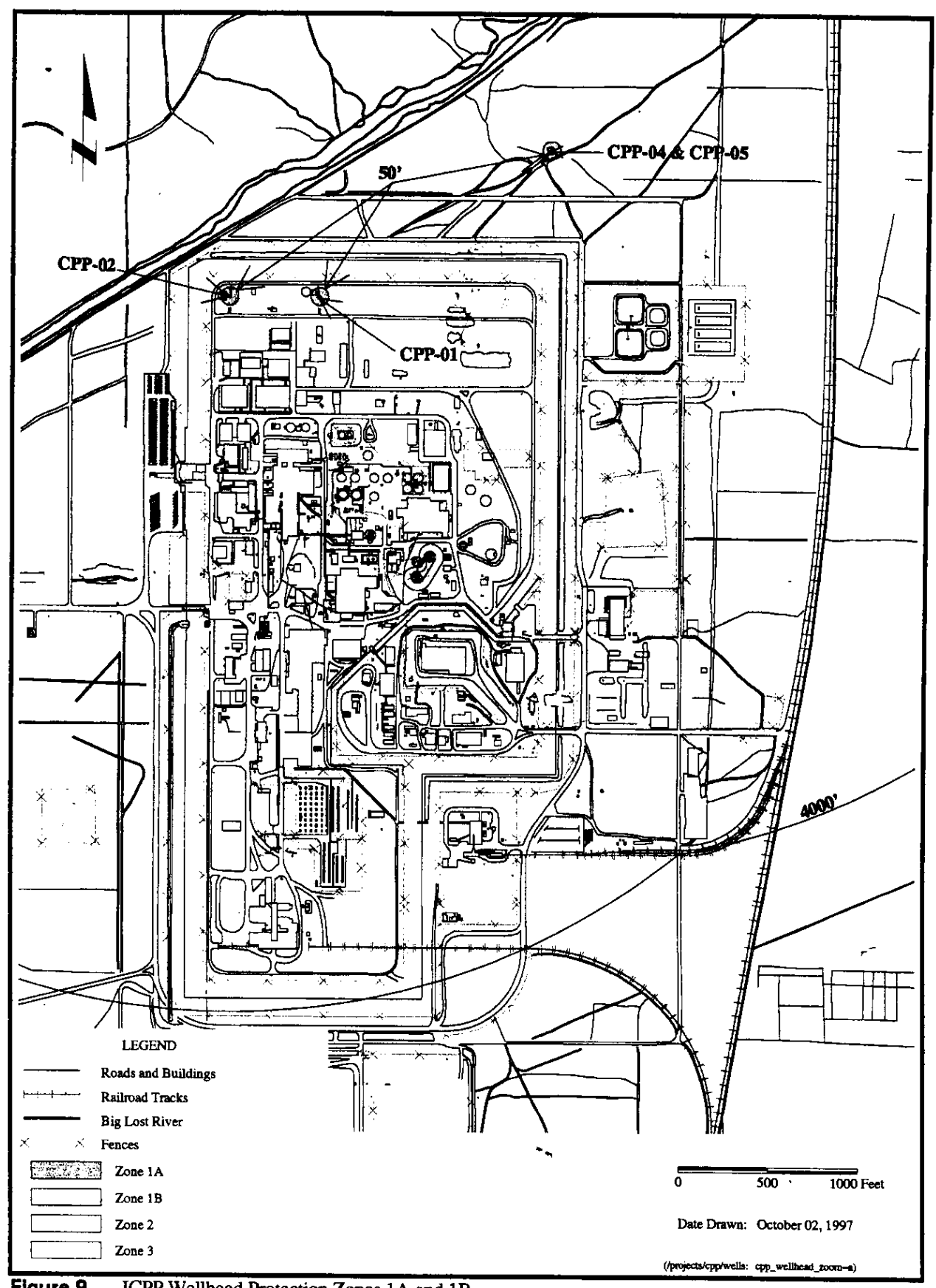

Figure 9. ICPP Wellhead Protection Zones $1 \mathrm{~A}$ and 1B. 


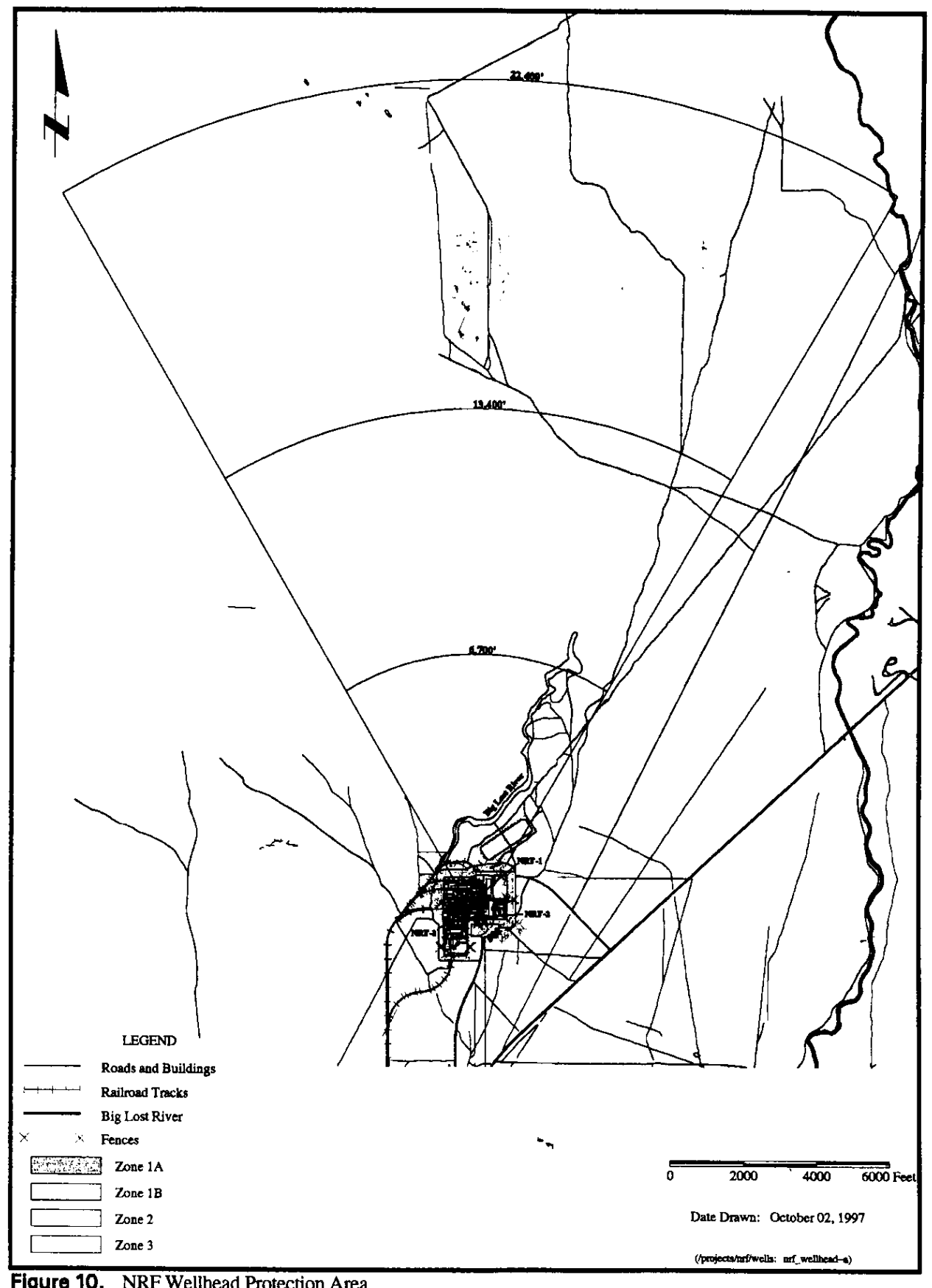

Figure 10. NRF Wellhead Protection Area. 


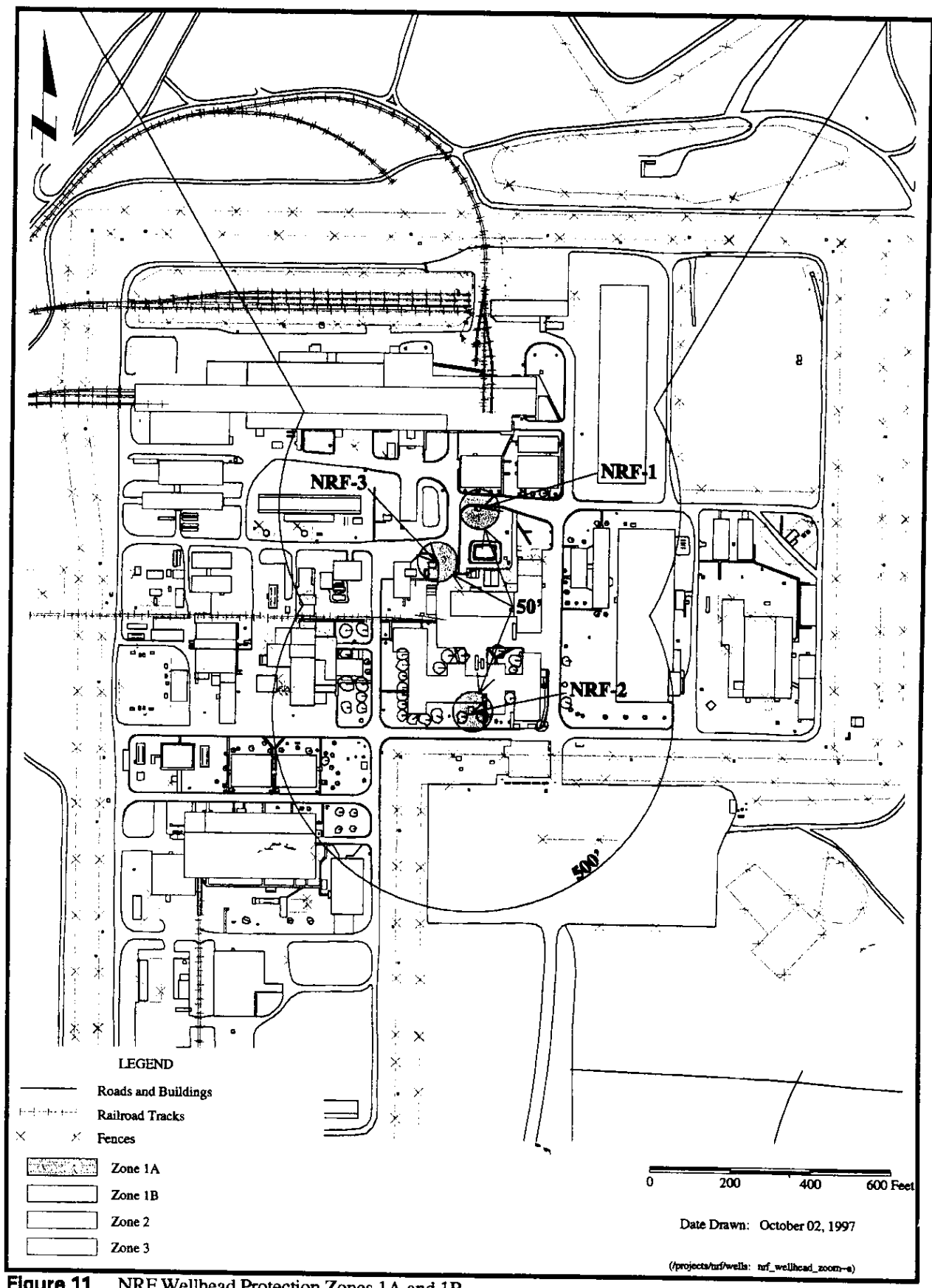

Figure 11. NRF Wellhead Protection Zones $1 \mathrm{~A}$ and $1 \mathrm{~B}$. 


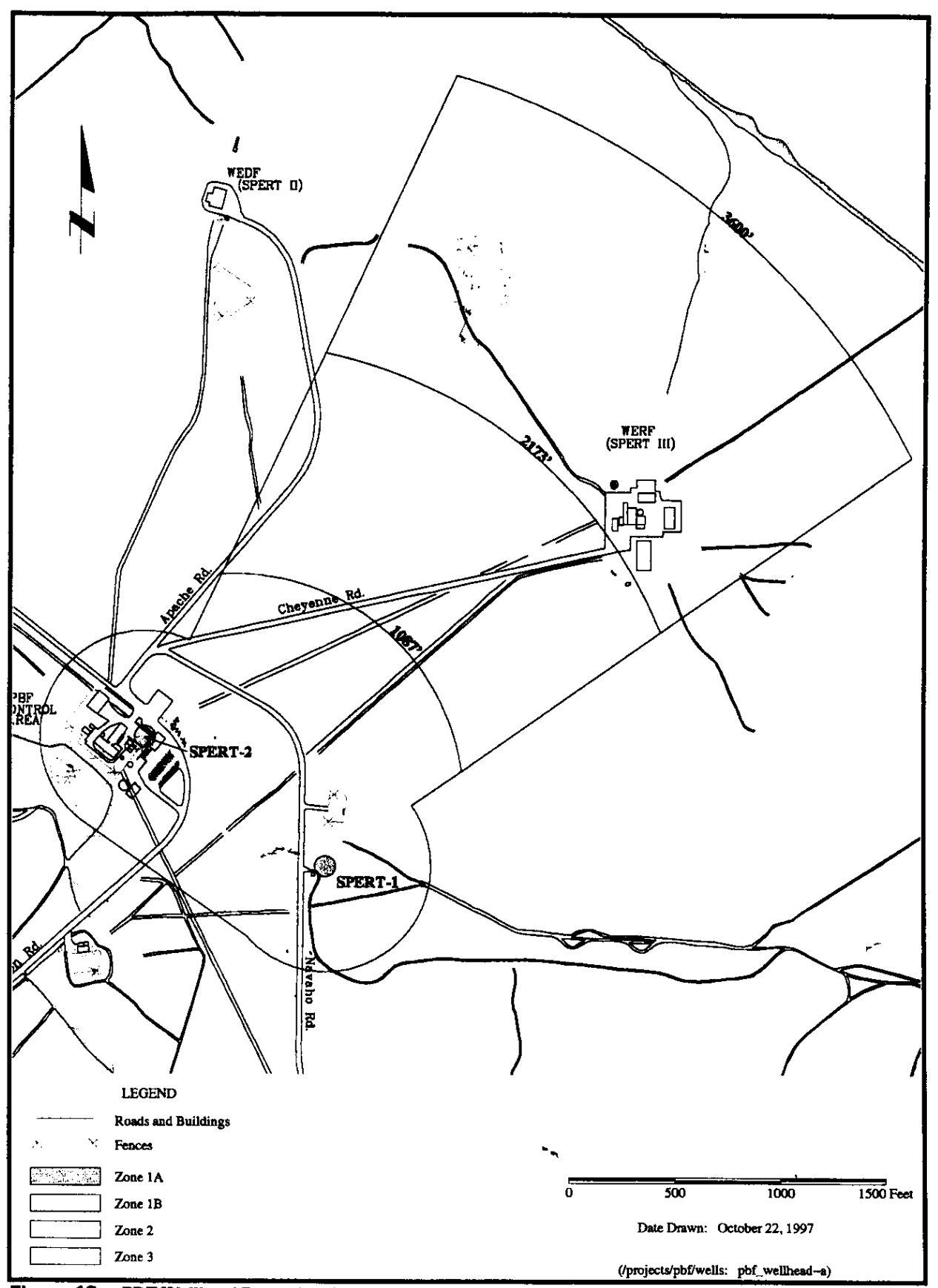

Figure 12. PBF Wellhead Protection Area. 


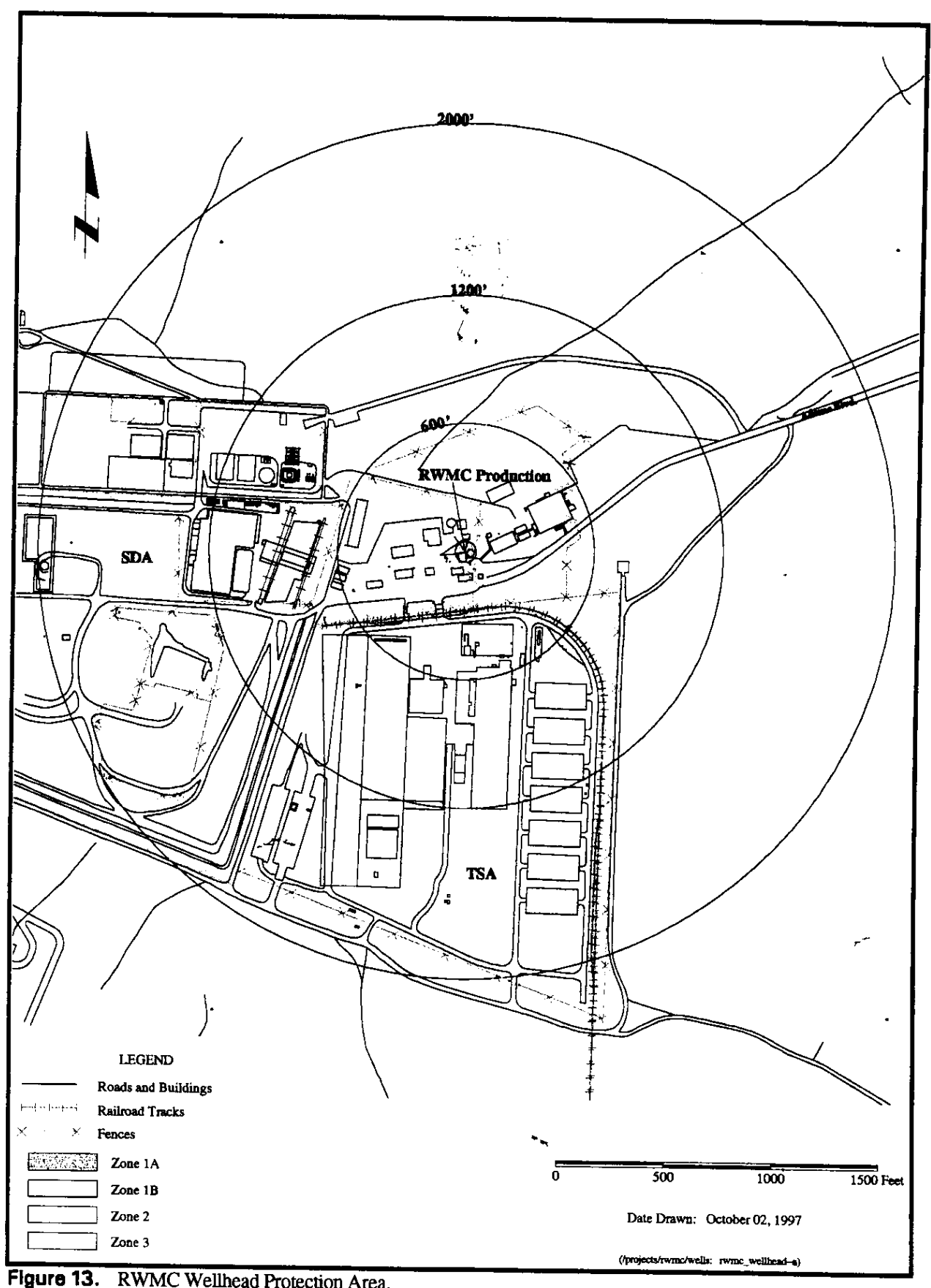

Figure 13. RWMC Wellhead Protection Area. 


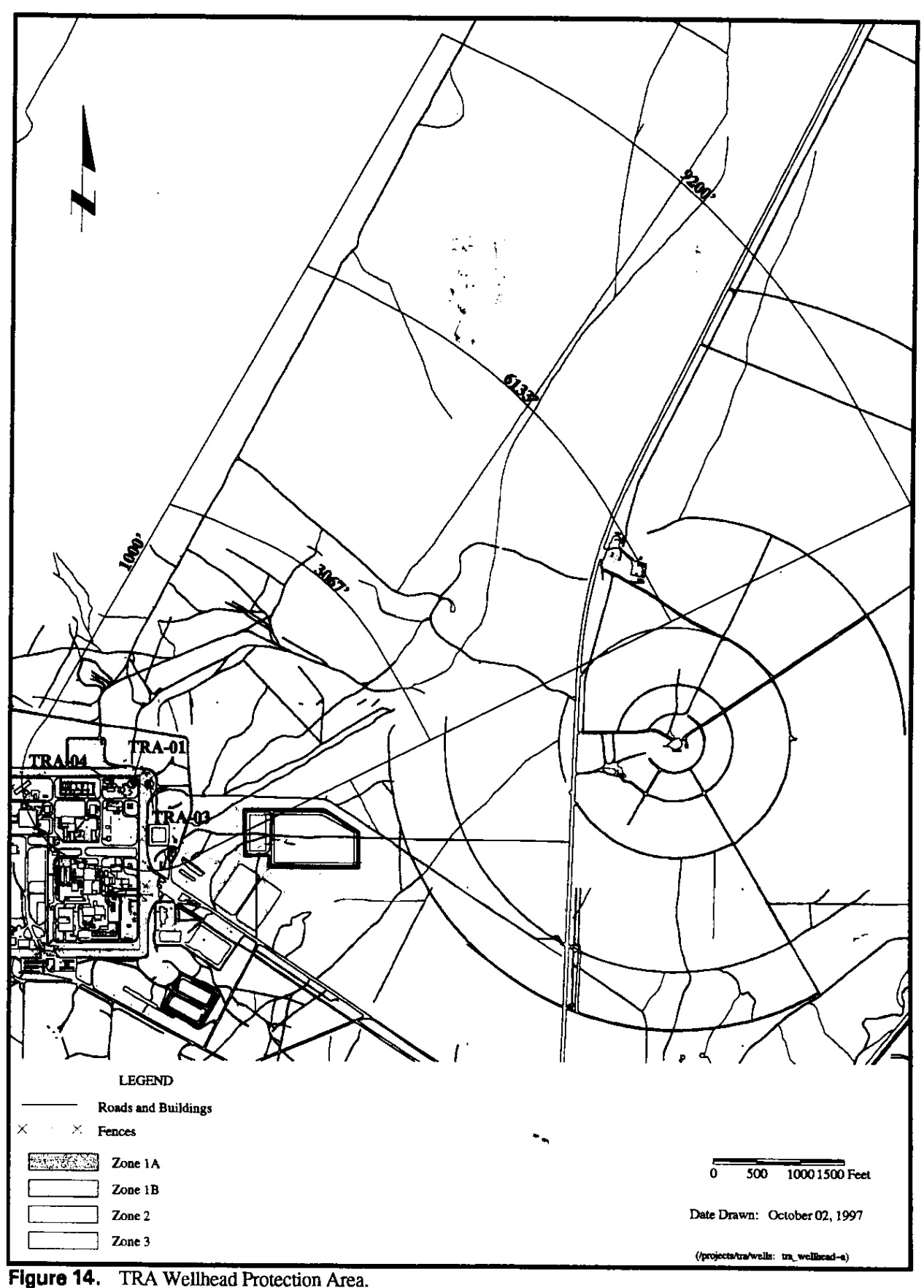

Figure 14. TRA Wellhead Protection Area. 


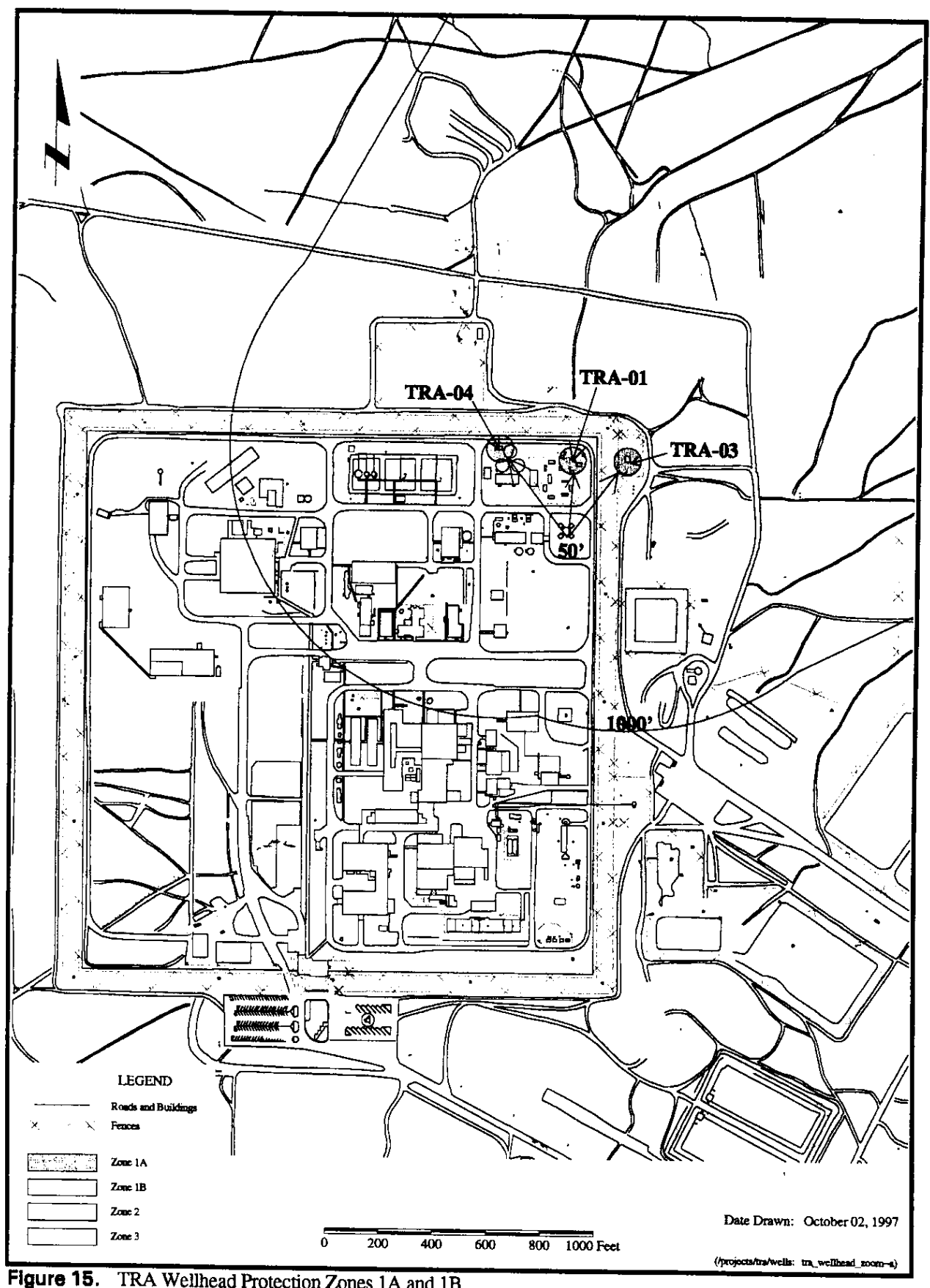

Figure 15. TRA Wellhead Protection Zones $1 \mathrm{~A}$ and $1 \mathrm{~B}$. 


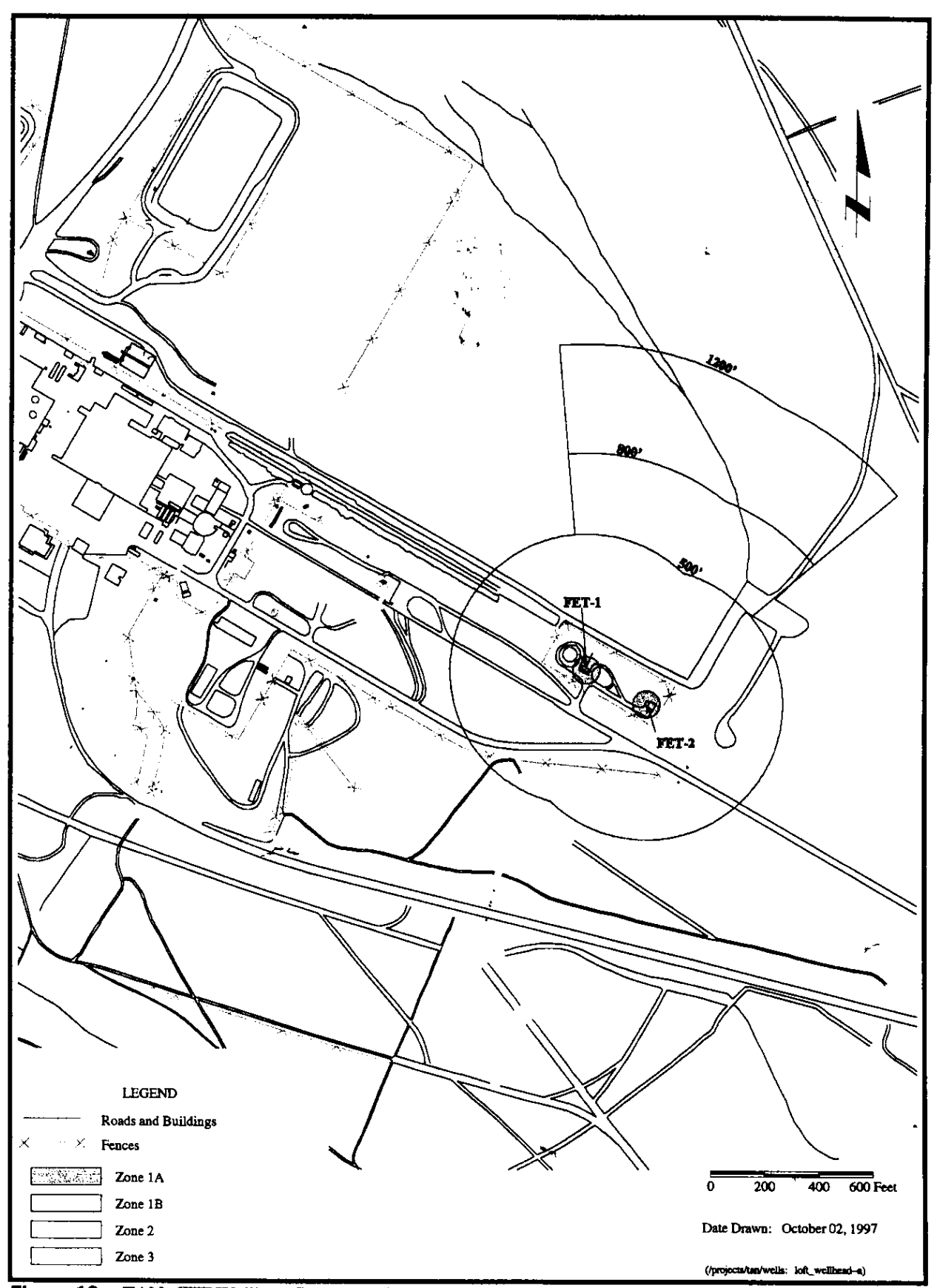

Figure 16. TAN-CTF Wellhead Protection Area. 


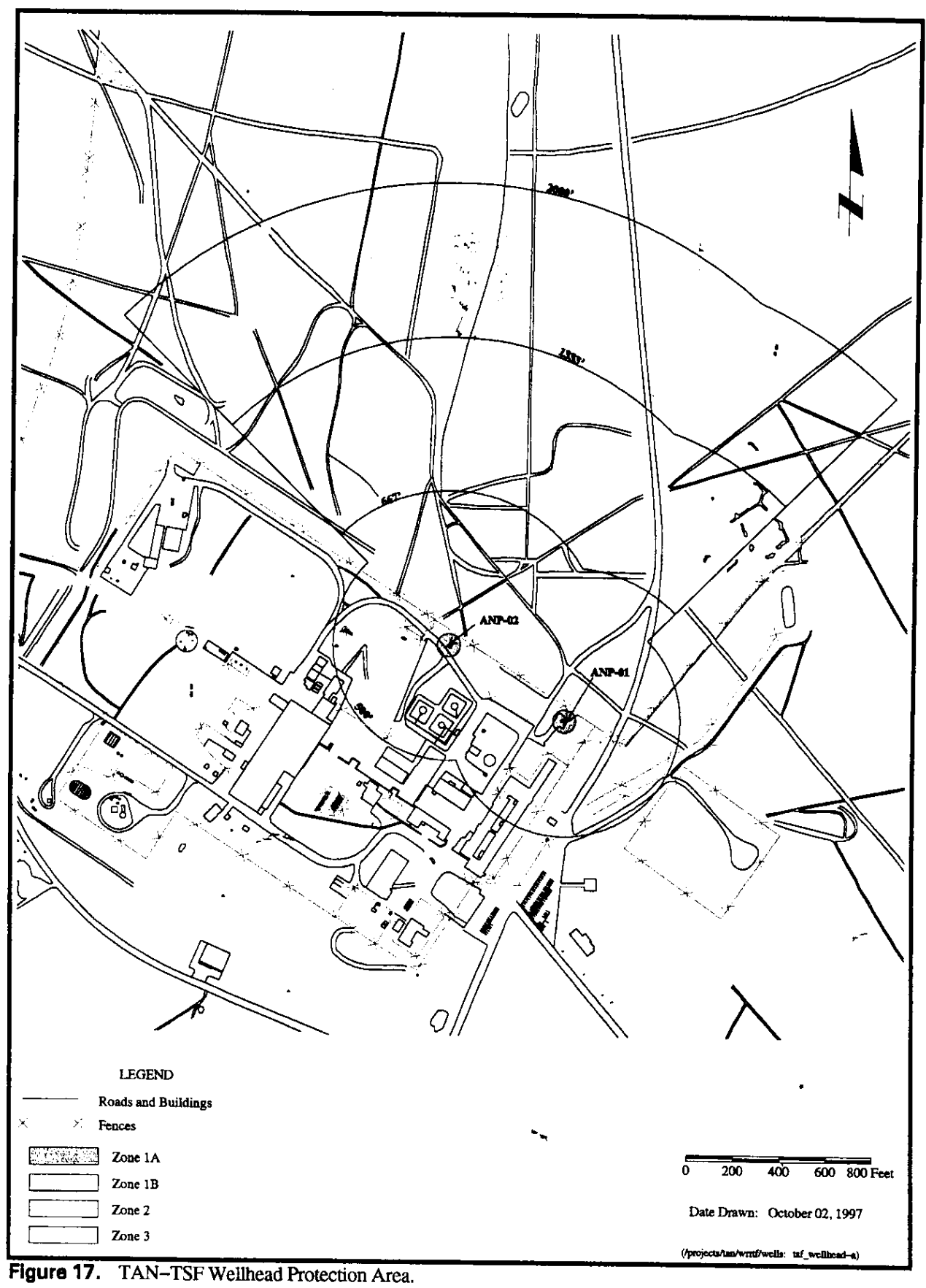

Figure 17. TAN-TSF Wellhead Protection Area. 


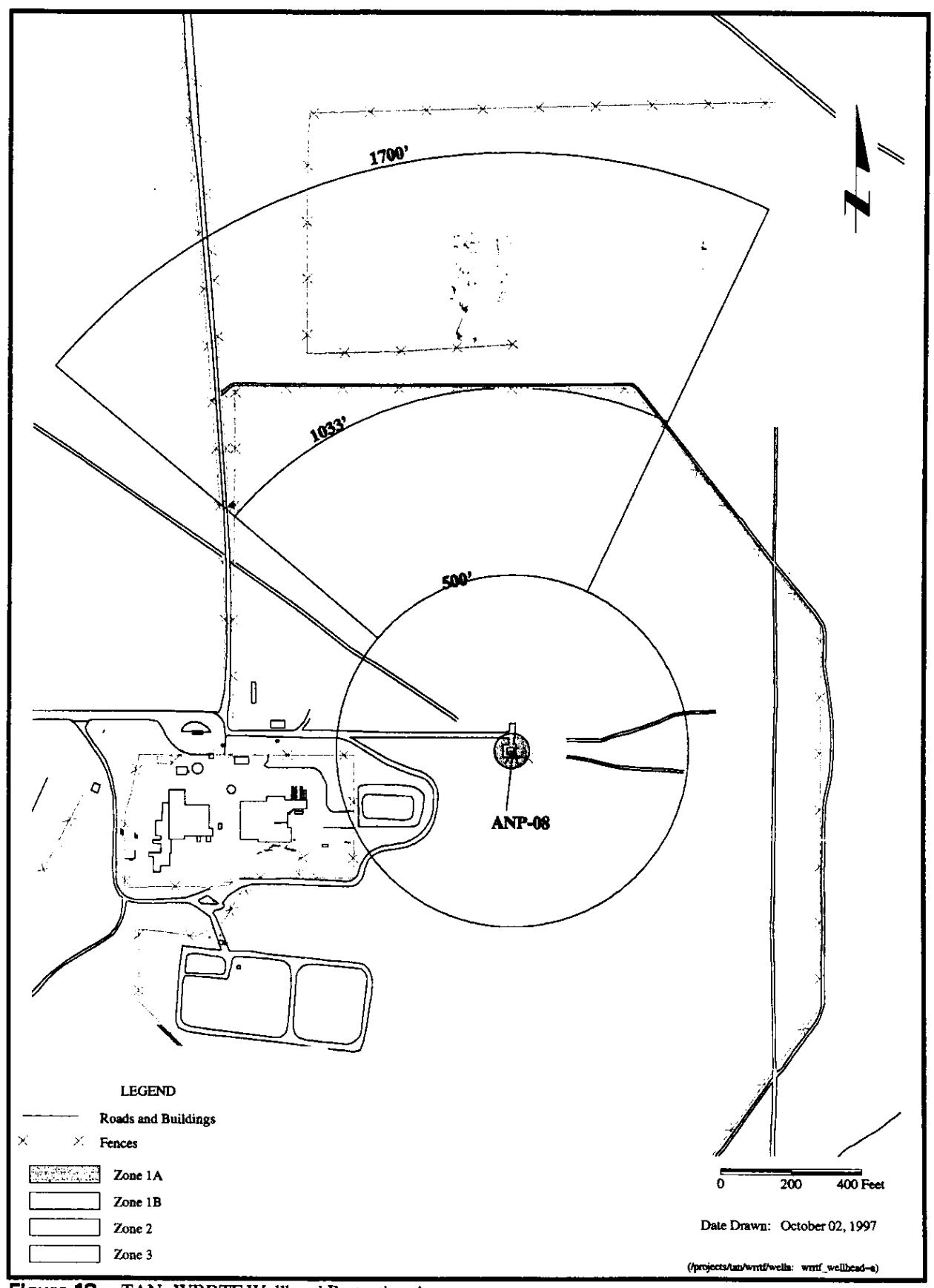

Figure 18. TAN-WRRTF Wellhead Protection Area. 
D-30 


\section{Appendix E}

\section{Environmental Checklist}

E-1 
E-2 


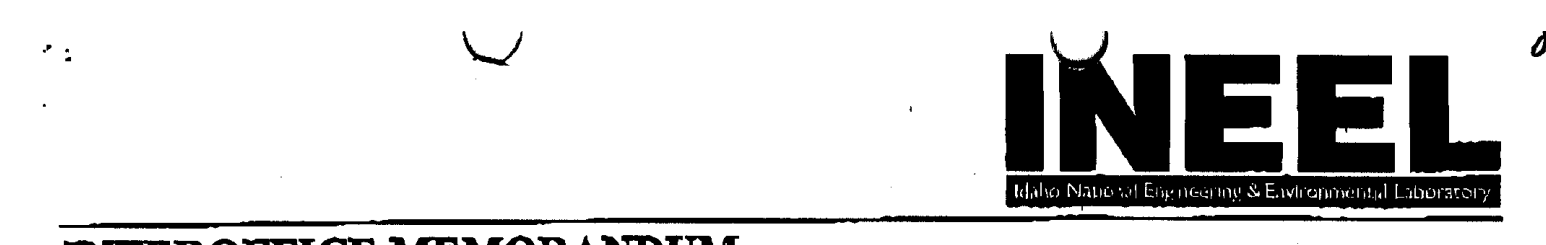

INTEROFFICE MEMORANDUM

$\begin{array}{ll}\text { Date: } & \text { October 6, } 1999 \\ \text { To: } & \text { J. K. Winterboller } \\ \text { From: } & \text { J. F. GTaham } \\ \text { Subject: } & \begin{array}{l}\text { APPROVAL OF THE DTEC PERCOLATION POND REPLACEMENT PROJECT (INTEC-99. } \\ \text { OP8) - JFG-105-99 }\end{array}\end{array}$

Bechtel BWXT Idaho LLC's (BBWTs) National Environmental Policy Act (NEPA)/Environmental Program staff has approved the attached Environmental Checklist (EC) for the proposed action. In addition, the DOE Environmontal Restoration Program Office and DQE NEPA Compliance Office have approved the action in accordance with the June 1994 Secretarial Policy on the National Environmental Policy Act. The attached BC and Approval Form will give your project an audit record and you may proceed with tho project subject to compliance with any conditions stated on the approval sheet. If the project description or scope changes, please notify B. E.

Walker at 526-8195. Reference the EC title and number (identified above) for subsequent transmittals regarding this EC.

BEW

Attachmonts

cc: K, M. Davis, MS 3921

J. D. Griffin, MS 4143

J. S. Irving, MS 3428 OSO

K. L. Miller, MS 5117

M. Vorachek, MS 5208

B. E. Walker, MS 4143

J. M. White, MS 5111

E. J. Ziemianski, DOE-ID, MS 5121

NEPA File INTEC-99-008

J. F. Graham File

UFC 6101/EA CFL-1 


\section{Environmental Checklist Approval Form}

ProjectNo.i INTBC.99-008

Project Title: DNTEC Percolution Pond Replacement Project

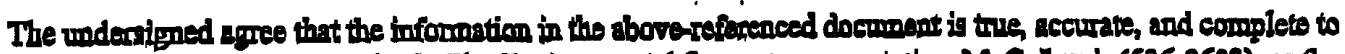
the best of their leowiedge. The ficility Envirommeath1 Support reprosentedve, M. G. Lewis (526-0623), of the Feoilty ES set Mamager, M. Vornethek (526-6925), should be contacted to deteming any addtional enviranenal requirementa. The condition lintsd below must be complefed before initheting tho proposed activity. This listing is not intended to be a bisting of envirenmental requtrements thas must be met while condreting the extivity.

$$
\text { geotechnical investigations) Bew }
$$

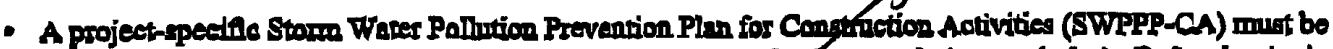

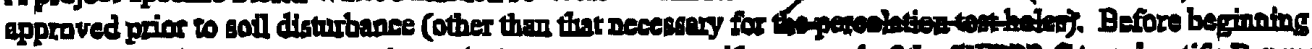

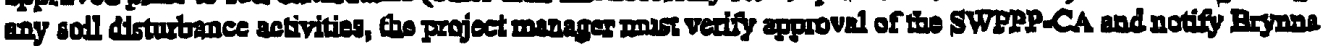
Walker (526-8195) when thils condition has been met,

Author:

Techmical Reviower:

Project Manager.

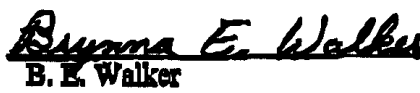

$\frac{2 / 2 y / 4 x}{10}$

B. En Walker

$2 / 27 / 2$ Jiplaving

Dato

* ouncucucole?

A. S. Fowy

Froility Operutions Manager:
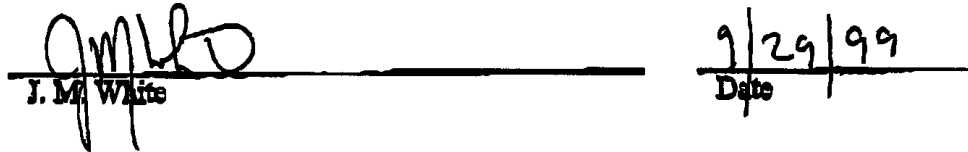

DOE Bnyironmentel Restorntion

Progman Ofice: DOE-ID NEPA Camplinnce
Qffice:

$$
\text { E }
$$

E-mail expraf Degperschmidt atwed

Atr/Whters NEPA Policy ond Perrituter:
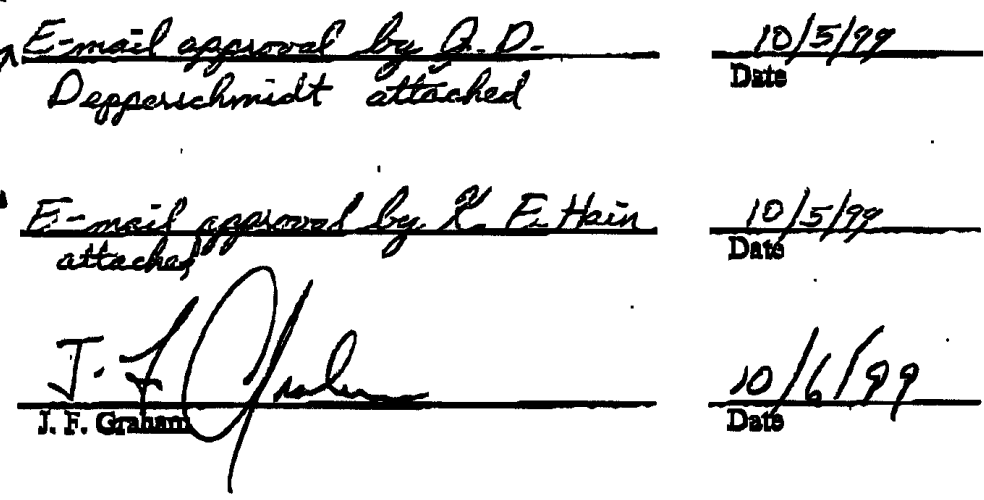
From: Jack D Depperschmidt@Exchange on 10/05/99 12:15 PM

To: Brynna K Evans/EVANBK/LMITCO/INEEL/US@INEL

cci John S Irving/JSI4/LMITCO/NEL/NS@INEL, Rachel L Collins@Exchange, Kathleen E Hain@Exchange, Talley W Jenkins@Exchange, Roger L Twirchell@Exchange, Vicki L Johnson@Exchange

Subject: FW: INTEC-99-008, Percolation Pond Replacement Project

Based on my review of the attached environmental checkllst, INTEC-99-008, I have determined, as NEPA Compliance Officer (as authorized by DOE Order 451.1A), that the environmental impacts of the proposed action should be covered by the CERCLA process as referenced in the text. Therefore, no further NEPA review is required

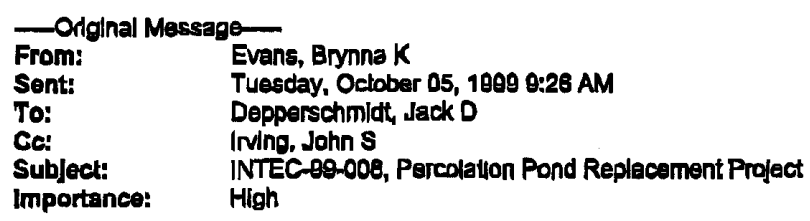

The final environmental checklist is attached for your NEPA approval. Thanks.

INTEC-DO-SOR,A00 
From: Kathleen E Haln@Exchange on 10/05/99 12:58 PM

To: Brynna K Evans/EVANEK/LMITCO/INEEL/US@INEL

cc:

Subject: RE: INTEC-09-008, Percolation Pond Replacement Project

Bryanna -

This EC is approved.

Kathleen E. Hain, Director

Environmental Restoration

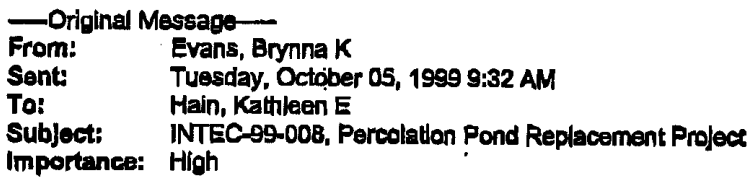

The attached Environmental Checklist (listed above) is being sent for your review of the activity and concurrence that it meets the requirements specified in the 1994 Secretarial Policy on the National Environmental Policy Act for a CERCLA activity that is excluded from the NEPA process. These requirements are:

1. That the proposed action does not site, construct, or operate a treatment, storage or disposal facility that, in addltion to supporting CERCLA actions, would also servo waste management or other purposes.

2. That steps have been taken to the extent practical to ensure opportunities for early public involvement in the CERCLA process and that DOE will make CERCLA documents avallable to the public as early as possible.

This EC has already been reviewed by the DOE-ID NEPA Compliance Office. All comments to date heve been resolved. The EC is in its final form, but has not been sent out as approved. If vou have any comments, please let me know. If you approve of the EC, please send me an e-mail note as soon as possible. Thank you.

\section{Brynna Walker}

$<<$ File: INTEC-99-008.doc > > 
451.01

$06 / 03 / 98$

Rev. 06
ENVIRONMENTAL CHECKLIST

U.S. DEPARTMENT OF ENERGY IDAHO OPERATIONS OFFICE

DIRECTIONS: Section A through D to be completed by the program/project manager. Section E \& F to be completed by the LMITCO's Policy and Permitting Organtzation, the DOE-ID NEPA Compllance Ofilcer (NCO), or as Indleated.

SECTION A. Project Title: INTEC Percolation Pond Replacement Project

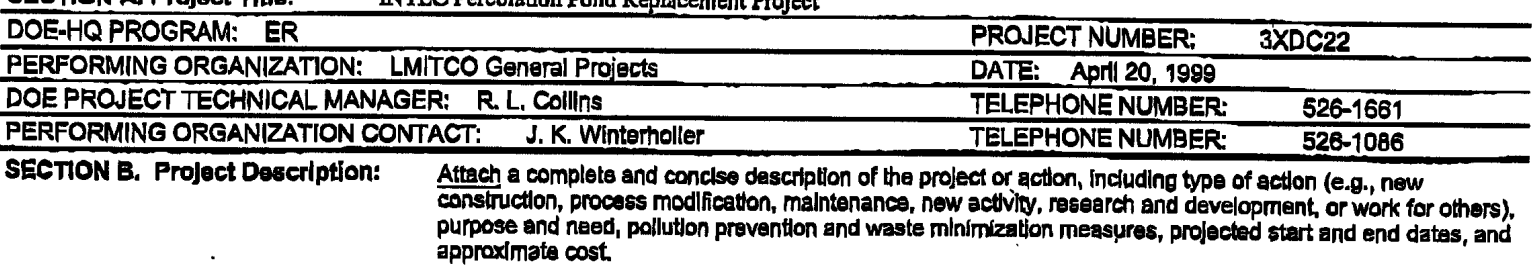

SECTION C. Sources of Impacts Would the action involve, genarate, or result in changes to any of the following? (If yes, explain on attactument,)

1. Alr Emisslons

2. Asbestos

3. Work Force Adjustment

4. Excess Nolsa Levels

5. Utillty Modification

6. Soil Disturbance

7. Water Treatment

\begin{tabular}{|c|c|}
\hline Yes & No \\
\hline$X$ & \\
\hline & $X$ \\
\hline$X$ & $X$ \\
\hline$X$ & \\
\hline$X$ & \\
\hline & $X$ \\
\hline
\end{tabular}

8. Water Source . WaterWell Use Water Course Modification 10, Pesticide Use 11. Chemical Use/Storage 12. Petroleum Storage 13. Solid Waste 14. PCBs

\begin{tabular}{|c|c|}
\hline Yes & No \\
\hline$X$ & \\
\hline & $X$ \\
\hline & $X$ \\
\hline & $X$ \\
\hline$X$ & \\
\hline & $X$ \\
\hline
\end{tabular}

Source 15. Hazardous Waste 16. Radioactive Waste 17. Mixed Wasto 18. Radiation Exposure 19. Liquid Effluent 20. Sensitve Resources 21. CERCLARCRA Site

\begin{tabular}{|c|c|}
\hline Yes & No \\
\hline & $X$ \\
\hline$X$ & \\
\hline & $X$ \\
\hline$X$ & \\
\hline$X$ & \\
\hline$X$ & \\
\hline$X$ & \\
\hline
\end{tabular}

SECTION D. The Action is Determined as: (check one of the following or if unsure call Policy and Permitting)

Appendix A Actions

10 CFR 1021 Appendix A to Subpart D

Routlne Malntenance Actions

10 CFR 1021 Appendix B to Subpart D, Subsectlon B1.3

$X$ Further NEFA documentation Is required. Forward to Polley and Permitting for NEPA doterminatlon and emvironmental review.

$$
\text { COF FE FILLD OUT BY THE POLICY AND PERMITING ORCANIZATION }
$$

SECTION E. Category Evaluatton Criteria; Would the action... (if yes, explain on attachment.)

1. Require cultural, historical, or blologlcal clearances?

2. Potentlally Impact sensitive resources Identlfled In Item 1 above. Describe the mitigation plan.

3. Require or modify federal, state, or local permits, approvals, etc.?

4. Be inconsistent with any existing consent orders or agreements (l,e, FFA-CO, slte wide treatment plans, etc.)?

5. Create waste for which there is no dispositlon, Waste Generation Number, or Solid Hazardous Waste Determinatlon from the Weste Menagement Authority?

6. Require siting, construction, or modification of a RCRA or TSCA regulated faclilty?

\begin{tabular}{|c|c|}
\hline Yes & No \\
\hline$x$ & \\
\hline & $x$ \\
\hline$x$ & \\
\hline & $x$ \\
\hline & $x$ \\
\hline & $x$ \\
\hline
\end{tabular}

\section{SECTION F. NEPA Level of Documentation and Reference(s)}

CX: EA: EIS: $\quad$ CERCLA: $X$ Prevlously Approved NEPA Documents: Not Covered In 10 CFR 1021

Reference(8): in accordance with the June 1994 Secretarial Policy on the National Environmental Policy Act, the Department of Energy will rely on the CBRCLA process for review of actions to be taken under CERCLA. This will be documented in the Recond of Docision for the CRRCLA jemediation plan for Operable Unit 3-13

Note; For projects checked above as "CX" (Categorleal Excluslon) the proposed action must not: 1) threaten a violation of applicable statutory, requlatory, or permit requlrements for environmențl, safety, and health, Including requirements of DoE orders; 2) require s|ting and construetlon or malor axpansilon of waste storage, dlsposal, recovery, or treatment facillties; 9) disturb hareardous substances, pollutants, contaminants, or CERCLA-excluded petroleum and natural ogs products that pre-exist in the environment such that there would be uncentrolled or unpormitted releaces; 4) advarsely affeet onvironmentally sensittve resources. In addition, no extraordinary elreumstances related to the proposul extat whileh would afiect the elgnificance of the aetion, and the aetion ls not "connected" nor " ralated" (40 CFR $1508.25(a)$ (1) end (2), respectively) to other actions with potentfally or cumulathely slenificant impacts.

Note: The above paragraph does not apply to EA, EIS, or CERCLA rolated activilas.

Name: Brynna E. Walker

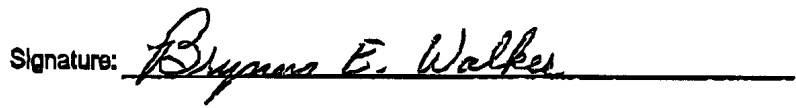

Telephone No.: 526-8195

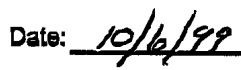




\section{ENVIRONMENTAL CHECKLIST \\ U.S. DEPARTMENT OF ENERGY

\section{ENVIRONMENTAL CHECKLIST ATTACHMENT}

\section{SECTION B (cont'd). Project Description:}

The proposed project will construct two percolation ponds at the Idaho Nuclear Technology and Engineering Center (INTEC), Idaho National Engineering and Environmental Laboratory (INEEL), to replace the existing percolation ponds. INTEC generates 1.5 to 2.5 million gallons of process wastewater (service water) per day. This water is currently discharged to two percolation ponds just south of DNTEC. The ponds are scheduled for closure under the proposed Comprehensive Environmental Response, Compensation and Liability Act (CERCLA) remediation plan for Operable Unit (OU) 3-13. The closure of the old ponds will be covered by a separate environmental checklist. This closure is required because water infiltration from the ponds can mobilize contaminants contained in the soil and perched water below INTEC. New ponds must be constructod before the existing ponds can be closed.

Two new like-for-like percolation ponds will be constructed at a new location approximately 2 miles southwest of the current ponds (see attached map). Two 14-inch diameter lines will be tied to the existing discharge lines and will rum approximately 13,000 feet from the tie-in point near the southwest comer of INTEC to the new point of discharge at the new ponds. A new road will be required to access the ponds.

The existing pumps (4 electrical and 1 diesel) and piping inside INTEC facilities CPP-797 and CPP-1 749 will be replaced to provide adequate capabilities to pump the extra distance to the now ponds. The existing pumps will be excessed. In addition, the project will require new groundwater characterization wells, groundwater monitoring wells, perched wates monitoring wells, and geoteclnical borings and excavations for percolation testing.

The proposed project is budgeted at \$4M. Geotechnical investigations (soil borings, percolation tests, and groundwater characterization wells) will begin in October 1999. The results of these investigations will be used as the basis for design and will also be required for the Wastewater Land Application Permit. Construction is scheduled to begin in March 2000 and be completed in November 2000. A subcontractor will perform all construction activities.

SECTION C (cont $\left.{ }^{2} d\right)$. Sources of impacts: Would the action involve, generate, or result in changes to any of the following?

1. Air Emissions - Trenching and excavation activities may generate fugitive dust emissions and exhaust from heavy equipment. See Section F, Requirements of Operations, No, 1 for guidance.

In addition, an emergency diesel pump will be replaced with a larger capacity pump in CPP-1749. See the attached Air Permitting Applicability Determination (APAD) for requirements of operations related to the diesel pump.

4. Excess Noise Levels - Excessive noise levels will be generated by heavy equipment portable compressors and powered hand tools. Personnel noise exposure will be managed by adhering to the requirements specified in MCP-2719, "Hearing Conservation," which implements the requirements of 29 CFR 1910.95 and 29 CFR 1926.52. Noise exposure managed in accordance with MCP-2719 is considered an insignificant impact on the human environment.

5. Utility Modification - The service waste system discharge line will be rerouted from the existing percolation ponds to the new percolation ponds. Two new 14-inch lines will be tied to the existing discharge lines and will extend approximately 13,000 feet from the existing point of discharge to the new percolation ponds. No new capacity will be added to the system.

Additional electrical energy will be required when the pumps inside CPP-797/1749 are changed out for larger pumps. Power will be provided for the new pumps from Load Center \$3, which is being installed east of CPP-797 by the Electrical Utility System Upgrade project. All modification activities will be conducted in accordance with DOE Orders, the National Electric Code, and the Occupational Safety and Health Act. 
6. Soil Disturbance - Approximately 10 acres will be excavated for the new percolation ponds, which will be about 10 feet deep. The excavated soil will be used to create berms around the ponds. In addition, a trench (approximately 13,000 feet long, 5-8 feet wide, and 5-10 feet deep) will be excavated for the new service waste discharge lines. The lines will be routed under roads by boring. Soil excavated from the trench will be used as backfill. A new graveled access road (approximately 1 mile long) will extend from Portland Drive north to the new ponds.

Geotechnical investigations required for title design will require drilling bore holes (about 4 inches in diameter by 15 feet deep) every 300 feet along the distribution pipe route and eight holes ( 4 inohes by about 40 feet deep (to basalt)) in the percolation ponds. Other geotechnical investigations will require digging four percolation test holes ( 4 feet in diameter by 4 feet deep). Following the geotechnical work the holes will be backfilled.

Four groundwater characterization wells will be drilled to the aquifer (approximately 500 feet deep) for Wastewater Land Application Permit baseline input. Four groundwater monitoring wells will also be required. The characterization wells will likely also be used for monitoring wells. The well locations are as follows: E285,459.438 and N687,396.563; E286,698.656 and N691,327.563; E287,491.375 and N687,177.063; and E285,855.719 and N690,620,688. Additionally, up to 5 perched water monitoring wells (about 150 feet deep) will be drilled during construction. The locations of these wells will be determined by the results of the characterization wells.

Excavation will also be required outside CPP-797 to change out a portion of an existing stainless steel header. Approximately 100 cubic yards of soil will be excavated and then used as backfill.

Excavation of the percolation test holes must be performed according to the INBEL Storm Water Pollution Prevention Plan for Industrial Activities (SWPPP-IA) (DOB/ID-10431). However, other soil disturbing activities must be performed according to an approved project-specific Storm Water Pollution Prevention Plan for Construction Activities (SWPPP. CA) (see Section F, Conditions, No. 1),

Note: The proposed location of the percolation ponds is not within the Big Lost River 100 year floodplain.

8. Water/Well Use - Potable water will be used to test the new discharge lines for leaks. In addition, the new percolation ponds will be designed to handle up to 3 million gallons of wastewater per day, since the rest of the system is designed to handie that quantity. The ponds will be sited to comply with a Wastewater Land Application Permit (WLAP) so that there will be no impacts on existing drinking water or production wells, or to perched water beneath INTBC (see Section, F, Requirements of Operations, No. 2). Overall plant water usage will not increase.

Within 8 days of well completion, well construction information must be submitted to the Integrated Earth Science Manger to update the INEEL Comprehensive Well Survey Database.

In addition, the wellhead protection plan developed by Environmental Affairs must be followed.

12. Petroleum Storage - Petroleum products such as gasoline, diesel fuel, motor oil, and hydraulic oils will be used during construction. They will be used in compliance with applicable contractor procedures.

13. Solid Waste - The proposed project will generate up to 30 cubic meters of nonhazardous, nonradioactive waste, such as packaging material, normal construction debris, and piping from demolition and tie-ins of the service waste lines. Upon waste generation. Waste Generator Services (WGS) will be responsible for performing hazardous waste determinations and waste characterization. This characterization will be completed according to WGS published procedures using the Waste Determination \& Diposition Form, INBEL form number 435.39. All weste generated and characterized will be managed to meet the requirements specified in the INEEI Reusable Property, Recyclable Materials and Waste Acceptance Criteria (RRWAC). Management of the waste will initially be the responsibility of the project manager and then transferred to WGS. 
16. Radioactive Waste - The proposed project will generate up to 3 cubic meters of radioactive waste, which would consist of hand tools and anti-contamination clothing used during the tie-in of the new discharge lines to the existing discharge lines inside INTEC and outside CPP-797/1749. Radioactive waste will be disposed of at the Radioactive Waste Management Complex (RWMC) according to the facility's WAC-RRWAC. Management of the waste will initially be the responsibility of the project manager and then transferred to WGS.

18. Radiation Exposure - There will be no more than 3 mrem total exposure during the tie-in of the new discharge lines to the existing discharge lines. Radiation exposure other than background level is not expected for the remainder of the project. Exposure will be managed in compliance with 10 CFR 835 "Occupational Radiation Protection," and the as low as reasonably achievable (ALARA) principle using time, distance, and shielding. The radiation exposure for personnel conducting this work will comply with a Radiation Work Permit (RWP) that controls maximum exposure levels.

19. Liquid Effuent - Potable water will be used to test the new discharge lines for leaks. This water will then be discharged into the new percolation ponds. Once the new ponds are in operation, up to 3 million gallons of wastewater per day will be discharged into tho ponds. This figure is based on historical records; there are no plans to actually discharge this much. The wastewater will be in compliance with all requirements of the WLAP permit.

By the time a new WLAP facility (i.e., the new percolation ponds) is 50 percent complete, the project manager must prepare a detailed plan of operation and submit to Environmental Affairs.

The project manager must submit to Environmental Affairs an as-built certification by a registered professional engineer and specifications within 12 working days of completing actual construction of the percolation ponds, or as-built plans if the actual construction deviates from the approved plans and specifications.

The project manager must submit a revised plan of operation to Environmental Affairs within 1 year of commencing operations.

20. Sensitive Resources - Ecological evaluations of the project areas have been performed by the Environmental Science and Ressarch Foundation (see attached letters from R, D. Blew). The areas being affected by this project are not wetlands, unique habitats, or areas known to be occupied by threatened or endangered species. It is unlikely the project will have a measurable impact on species of federal or state concern. Percolation pond berms will be sloped to allow wild life to safely access the ponds to obtain drinking water. Fencing will not be installed around the new ponds. The distribution pipe excavation and pond berm sides will bo planted with nativo species.

Excavation for the new percolation ponds, groundwater characterization wells, geotechnical tests, access road, groundwater monitoring wells, perched water monitoring wells, and water distribution lines could be constructed near identified cultural sites. To avoid these sites, all soil disturbance activities should be coordinated with the INEEL Cultural Resource Management Office. Clearance has been recommended for this work (see attached notes from Brenda Pace and Clayton Marler).

21. CERCLA / RCRA Site - Excavation activities will be conducted inside Environmentally Controlled Area (BCA) CPP-88 for distribution line tie-ins and to replace approximately 5 feet of an existing stainless steel beader outside CPP797. The new percolation ponds will be located outside of INTEC and outside of any ECAs. This location will be included in the Record of Decision for Operable Unit 3-13.

SECTYON E. Category Evaluation Criteria: Would the action ... .

3. Require or modify federal, state, or local permits, approvals, etc.? Project-specific SWPPP-CA

SECTION F. NEPA level of documentation and reference(s). 
451.01

06/03/98

Rev, 06
ENVIRONMENTAL CHECKLIST

U.S. DEPARTMENT OF ENERGY IDAHO OPERATIONS OFFICE

Requirements of Operations:

1. Fugitive Dust Control - Fugitive dust emissions that may be produced during excavation and trenching activities must be controlled in accordance with IDAPA 16.01.01.650, "Idaho Rules for Control of Fugitive Dust." This requires that all reasonable precautions be taken to prevent the generation of fugitive dust. The performing organization project manager shall ensure that fugitive dust emissions are controlled. Some reasonable precautions may include, but are not limited to, using water or chemicals, using control equipment, and covering truciss. For additional guidance, see the attached APAD or contact John Gill, LMITCO Environmental Affairs, at 526-8406.

2. Wastewater Land Application Permit - A WLAP must be obtained to allow service wastewater to be discharged into the new percolation ponds. For guidance, contact Dennis Walker at 526-0226.

\section{Conditions:}

1. Soil Disturbance - A project-specific Storm Water Pollution Prevention Plan for Construction Activities (SWPPPCA) must be approved prior to soil disturbance (other than that necessary for geotechnical investigations). Other work, such as Title Design, may be conducted before the SWPPP-CA is approved. For guidance, consult the INEEL SWPPPCA document (DOE/ID-10425). If additional information is needed, contact the INEEL SWPPP Coordinator, DeAnne Braun, at 526-8409. Before beginning any soil disturbance activities, the project manager must verify approval of the SWPPP-CA and notify Brynng Walker (526-8195) when this condition has been met.

\section{Contractor Comments:}

1. Summary of the Proposed Action: The proposed project will construct two percolation ponds at INTEC to replace the existing percolation ponds, which are scheduled for closure. Two new like-for-like percolation ponds will be construoted at a new location approximately 2 miles southwest of the current ponds. Two 14-inch diameter lines will be tied to the existing discharge lines and will nun approximately 13,000 feet from the existing point of discharge to the new point of discharge. The proposed project is budgeted at \$4M. Construction is scheduled to begin in March 2000 and be completed in November 2000. A subcontractor would perform all construction activities,

2. Use of the CERCLA Process: In accordance with the June 1994 Secretarial Policy on the National Environmental Policy Act, the Department of Energy will rely on the CERCLA Process for review of actions to be taken under CERCLA. The proposed activity supports a CERCLA action and does not support the siting, construction, or operation af a treatment, storage, or disposal facility for waste management or other purposes unrelated to CERCLA. The CERCLA documents for this activity have incorporated NEPA values to the extent practicable, and the CERCLA documents will be made available to the public in accordance with the requirements of CERCLA.

3. CERCLA Strategy: Although the proposed activity is not a CERCLA action, it does support a future CERCLA action, since the new percolation ponds must be constructed before the existing ponds are closed under CERCLA. This is documented in the Record of Decision for the CERCLA remediation plan for OU 3-13 (to be signed by all parties in October 1999). 


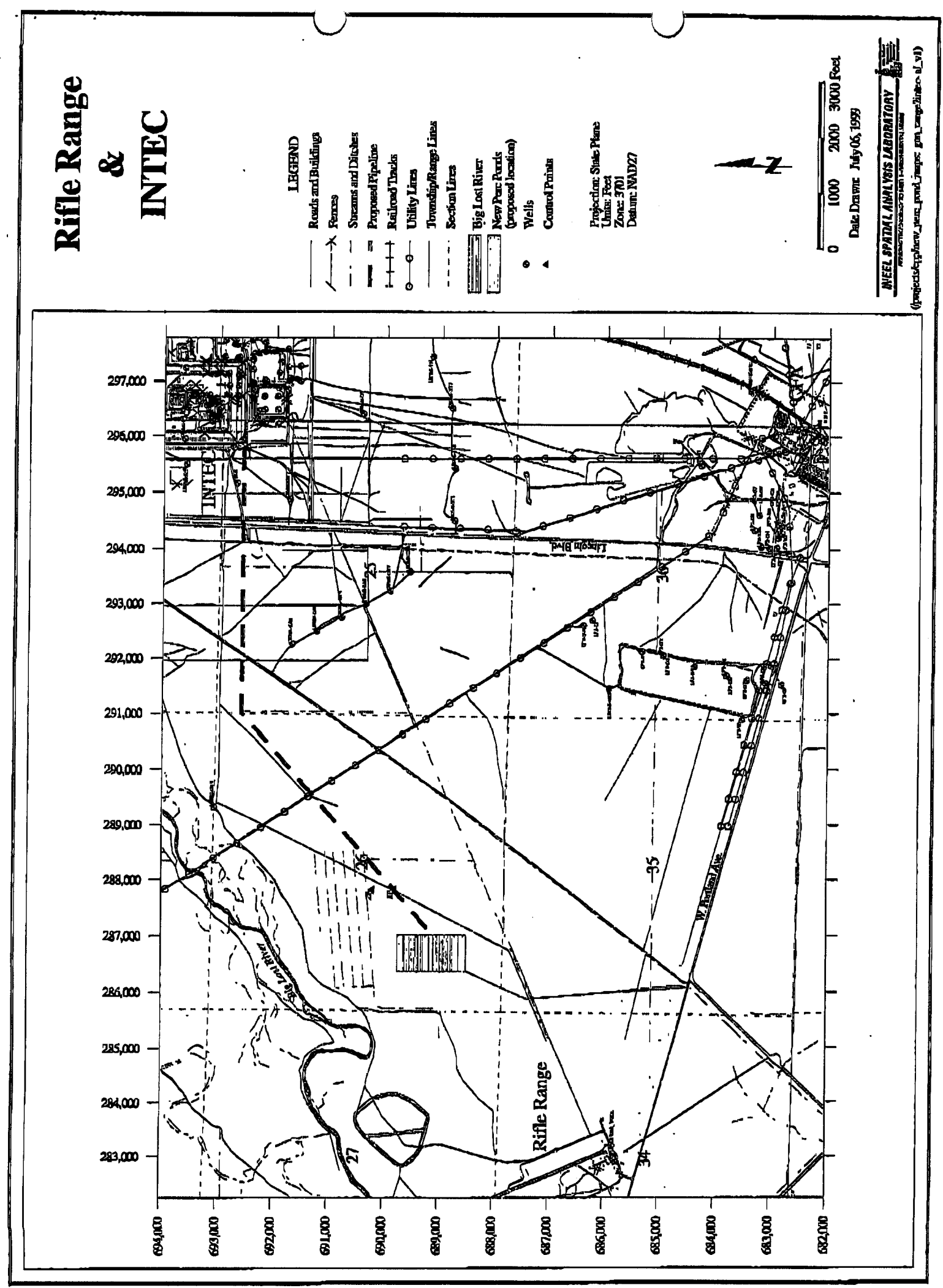




\section{AIR PERMITTING APPLICABIITY DETERMMNATION (APAD) ENVIRONMENTAL AFFAIRS}

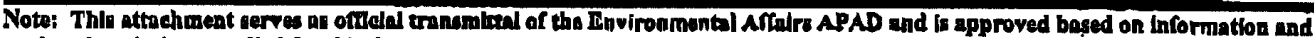
project deseription supplied for this detormination. The undersigned agree that ehe intormation in the reforenced docoment is true, acenrate and complete to the best of thelr knowledge.

Section A. Reviewer, Tracldng, and Approval

\begin{tabular}{|c|c|}
\hline \multicolumn{2}{|l|}{ Project Title: INTEC Percolation Pond Replacement Project } \\
\hline Date: Seprember 28, 1999 & Project Number: $3 \times \mathrm{XDC} 22$ \\
\hline APAD Tracking Number: 99-64 & NEPA Document Number: INTBC-99-008 \\
\hline $\begin{array}{l}\text { APAD Technical Author: Rachel Dolmose } \\
\text { Telephone: } 526-5950\end{array}$ & $\begin{array}{l}\text { Signature: } \\
\text { Rechel Delmore } \quad 9 / 2 \mathrm{~g} / 98\end{array}$ \\
\hline $\begin{array}{l}\text { APAD Technical Reviewer. Ray McDougal } \\
\text { Telephone: } 526-0517\end{array}$ & S Date: \\
\hline $\begin{array}{l}\text { Manager Approval (not requtred for transmittal of no } \\
\text { permitting required statements): E. Dennis Walker } \\
\text { Telephone: } 526-0226\end{array}$ & Siffature: \\
\hline $\begin{array}{l}\text { DOE Project Technical Manager (not requilred for } \\
\text { transmitral of no permitting regulred stetements): } \\
\text { James F. Graham Telephone: 526-7935 }\end{array}$ & Signanure: \\
\hline $\begin{array}{l}\text { Performing Organization Project Manager (not requitred for } \\
\text { transmittal of no permitting required statements)i } \\
\text { Kirk Winterholler Telephone: }\end{array}$ & Signature: \\
\hline $\begin{array}{l}\text { Facility Manager (not required for transmittal of no } \\
\text { permitting required statements): } \\
\text { Telephope: }\end{array}$ & Signature: \\
\hline
\end{tabular}

Section B. Air Permitting Applicability Determination

Peimit to Construct (PTC) required (contact DEO)
Prevention of Significant Deterioration (PSD) Permit
Category II Exemption
Further Evaluation for Permitting Required


Section C. Brief Description of Air Pollutant Emittling Aspects of Proposed Activity

Percoletion pond replacement will produce fugitive dust emissions during excavarion and trenching activities.

A $\leq 100$ horsepower emergency diesel pump will be installed and usad, however, Kirk Winterholler indicated that the pump will use diesel fuel, and ren less than one hour per mopth for testing purposes, which qualifies the pump for a Category II Permit to Construet (PTC) exemption.

Section D. Impact (check if applicable)

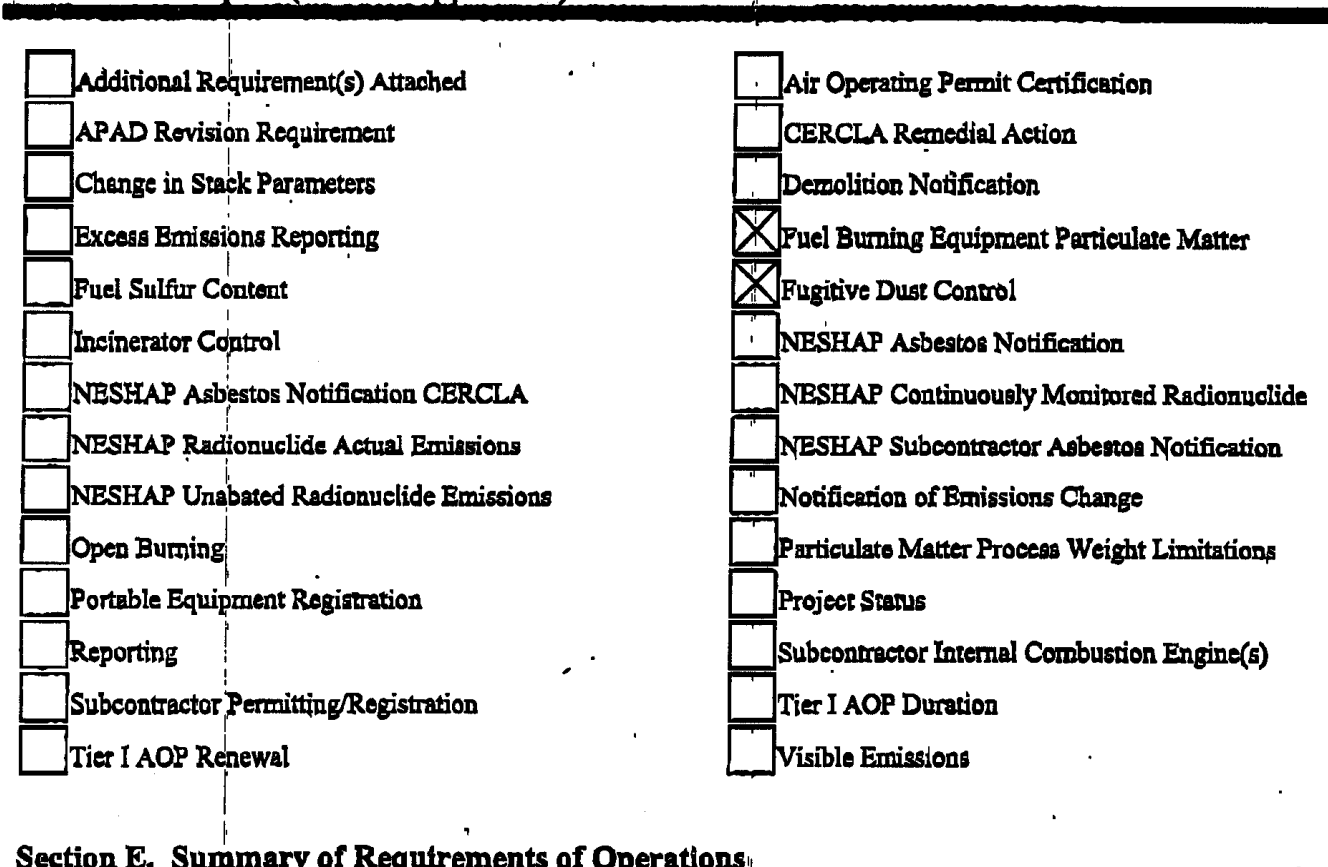

Section E. Summary of Requtrements of Operations:

Fugitive Dust Control - Fugitive dust emissions that may be produced during construction, demolition, excavation, and backfflling activities must be controlled in aceordanee with Idaho Administrative Procedures Act (IDAPA) 16,02,01,650, "Idaho Rules for Control of Fugitive Dust". This requires that all reasonable precautions be taken to prevent the generation of fugitive dust. The performing organizatios Project Manager shall ensure tha fugitive dust emissions for the proposed aetion are controlled, Some reasonable precautions may include but are not limited to, the use of water or chenricals, the use of control equipment, and the covering of trucks, For additional guidance, contact John Gill (LMITCO Envirommental Affhirs; 526-8406).

For combustion sources with a maximum rated input of less than 10 million BTU/hr or more particulate matter emissions shall pot exceed the grain loading emission limits of 0.015 grains per dry standard cubic foot (gr/dact) of effluent gas corrected to three percent ( $3 \%$ ) axygen by volumo for gaseous fuel, $0.050 \mathrm{gr} / \mathrm{dsef}$ for li quid fuel, and $0.100 \mathrm{gr} / \mathrm{dsef}$ for cosl (corrected to $8 \%$ oxyger.

For combustion sources with a maximum rated inpus of 10 million BirU/hr or more, particulate matter emissions shall not exceed the grain loading emission limits of 0.015 grains per dry standard eubje foot (gr/dsef) of effluent gas corrected to three percent (3\%) oxygen by volume for geseous fuel, $0.050 \mathrm{gr} / \mathrm{decf}$ for liquid fuell, and $0.050 \mathrm{gr} / \mathrm{dscf}$ for coal (corrected to $8 \%$ oxygen). IDAPA 16.01.01.675-677 
Sectlon F. Summary of Air Emissions Environmental Reports Performed by Environmental Affairs

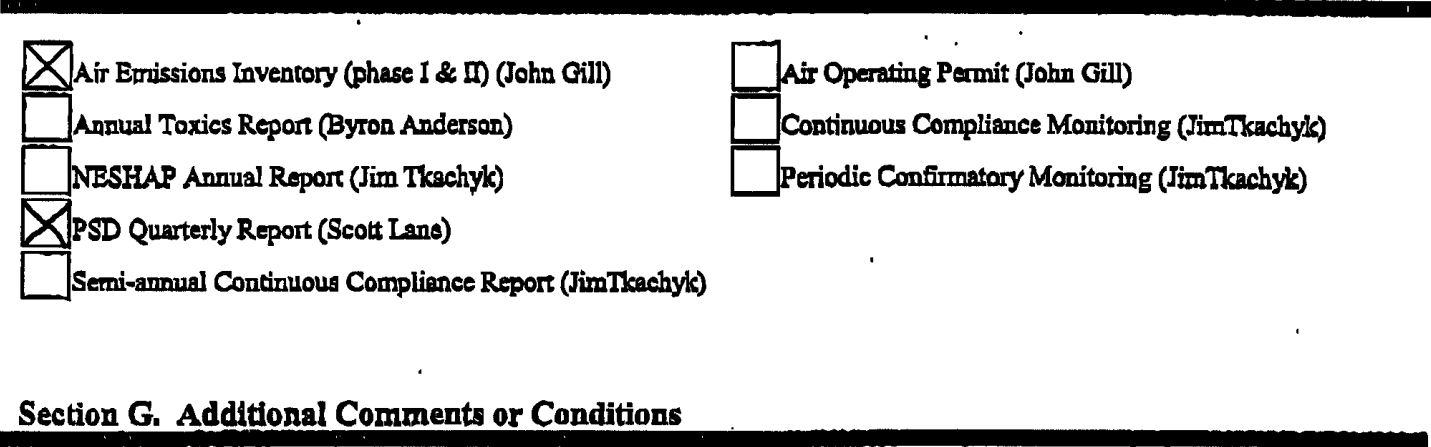

None

Section H. Summary of Air Operating Permit Requirements

$$
N / A
$$

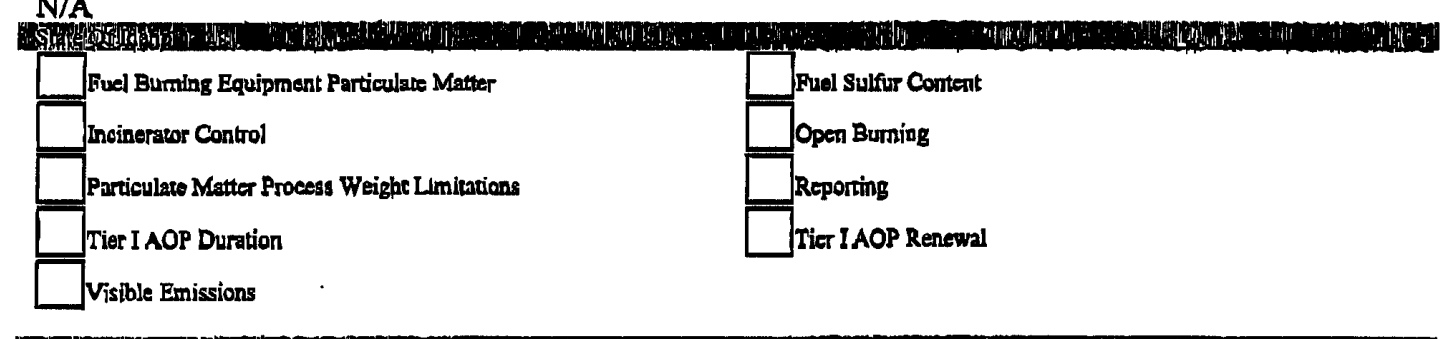

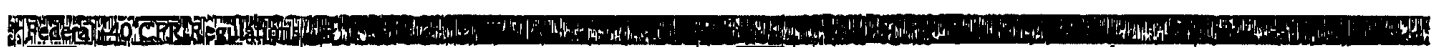

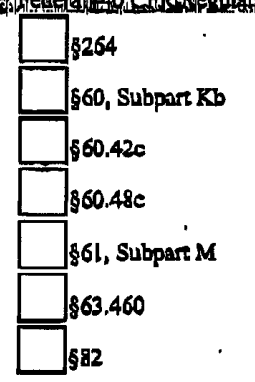

\begin{tabular}{l}
\hline 60. Subpart Dc \\
560.1160 \\
$\$ 60.440$ \\
561. Subpart H \\
$\$ 51.154$ \\
$\square 68$
\end{tabular}

Section I: Air Operating Permit Requirements

N/A 
Section J: Justlfication for APAD

Potential for fugitivo dust emissiobs with regard to excavation and trenching activities of percolation ponds. Also, the emergency diesel pump is a point-source.

\section{DAPA 16.01.01.220 GENERAL EXMMPTION CRITERIA FOR PERMIT TO CONSTRUCT FXIMPTIONS}

01. Gezeral Exemption Criteria Sections 220 hrough 223 may be used by owners or operators to exempt certain sources from the requirement to obtain a permit to construct. Nothing in these sections shall preclude an owner or operator from choosing to obtain a permit to construct. For purposes of Sections 220 through 223 , the term source means the equipmens or activity being exempted. No permit to construct is required for a sourec that satisfies all of tho following criteria, in addition to the critaria set forth at Sections 221, 222, or 223: (11-13-98)T

a. Less than, one hundred (100) tons, Uneontrolled potential emissions of the source shall not exceed one hundred (100) tons per year of any regulated air pollutene. (11-13-98)T

Qunlification: Uncontralled potential amlissions will be less than 100 tons per year.

b. No significant increases. Uncontrolled potontial enistions of the source shall not cause an increase in the emissions of a major facility that exceeds the significant enissions rates set out in the definition of aignificant at Section 006. (11-13-98)T

Quallification: Uncontrolled potential emissions will not cause a slgnificant increase. .

c. Compliance with NAAQS. Uncontrolled potential emissions of the source ghall not caure or significantly contribute to a violation of an ambient air quality atandard, based upon the applicable ais quality models, date bases, and other requirements of 40 CFR Part S1, Appendix W (Guideline on Air Quality Models). No further demonstrarion is required for those sources listed at Subsection 222.02. (11-13-98)T

Quallfeation: The 5100 horsepower emergency pump will use diesel fuel and will be operated less than

exceedance of the

two hundred (200) hours per year. This umited use will noc contribute to

NAAQS standards, (See Appendlx A for emission caleulations and summary.

d. Combination. The source shall not be part of a proposed new major facility or part of a proposed major modification. (11-13-98)T

Qualification: The source is not part of a new major facility.

02. Record Retention. Unless the source is subject to and the owner or operator complies with Section 385 , the owner or operator of the source, except for those sources listed in Subsections 222.02.a, through 222.02. g., shall maintain documentation on 'site which shall identify the exemption determined to apply to the soures and verify that fhe source qualifies for the identified exermption. The reconds and doeumentation shali be kept for a period of time nor less than five (5) years from the date the exemption determination has been made or tor the life of the source for which the exemption has been determined to apply, which ever is greater, or until such time as a permit to construct or an operating permit is issued which covers the operacion of the source. The owner or operator shall submit the documentation to the Departoment upon request. (11-13-98)T

Qualification: Required records wull be rotained.

\section{CATEGORY II EXEMPTION.}

No permit to construct is required for the following sources. (11-13-98)T

01. Exempt Source, A source that satisfies the criteria set forth in Section 220 and that is specified below: (11-13-98)T

d. Stationary intermal combustion engines used exclusively bor emergency purposes which are operafed less than two hundred (200) hours per year and are fueled by natural gas, propane gas, liquefied petroleum gas, distillate fuel oils, residual fuel oils, and diesel fuel; waste oil, gasoline, or refined gasoline shall not be used. (11-13-98)T

Qualifieution: Per Kirk Winterholler, the pump is for emergency purposes and qualifies for This exemption dae to the siza, usc of diesel foel, and limited yearly use. 


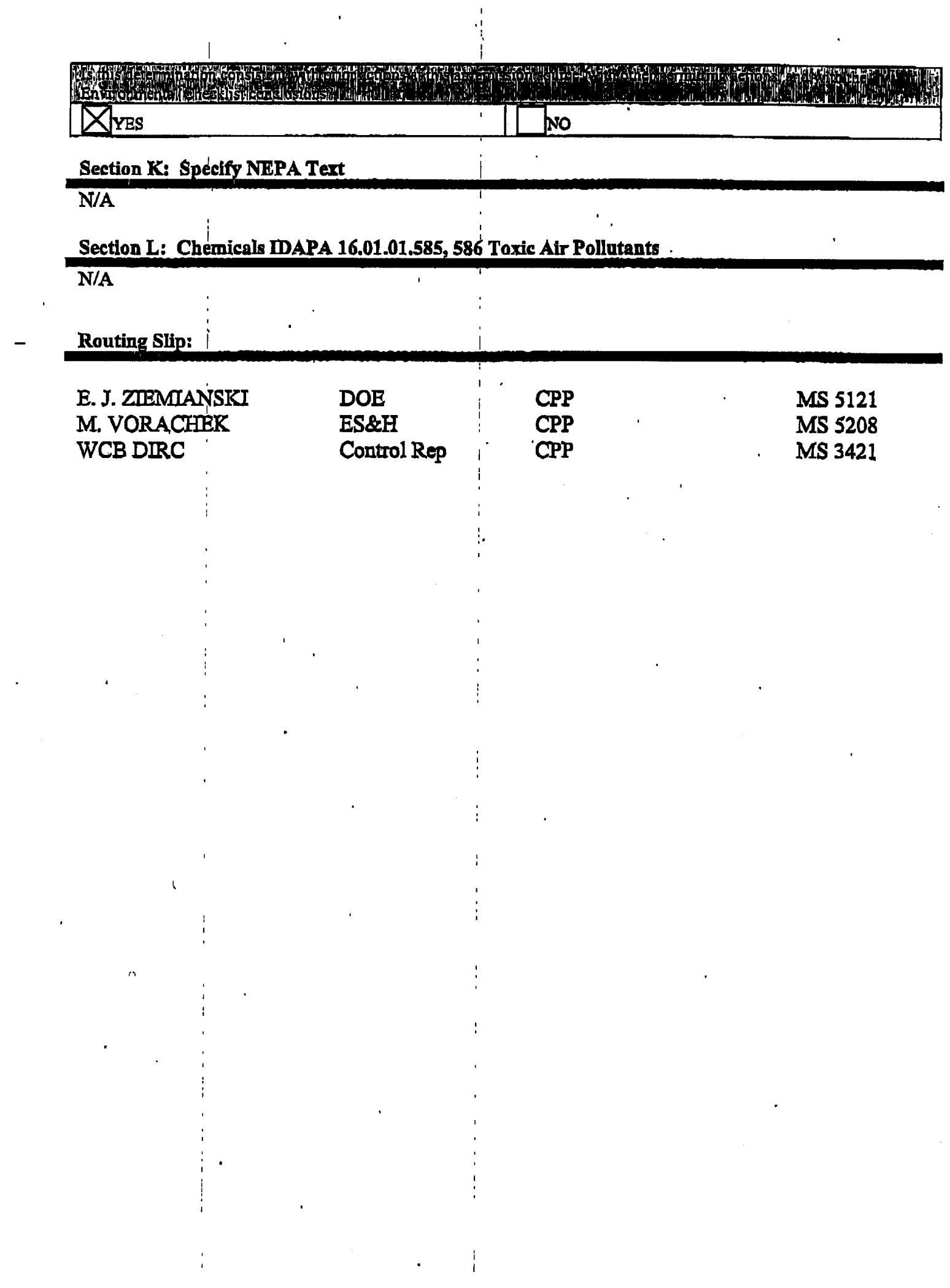




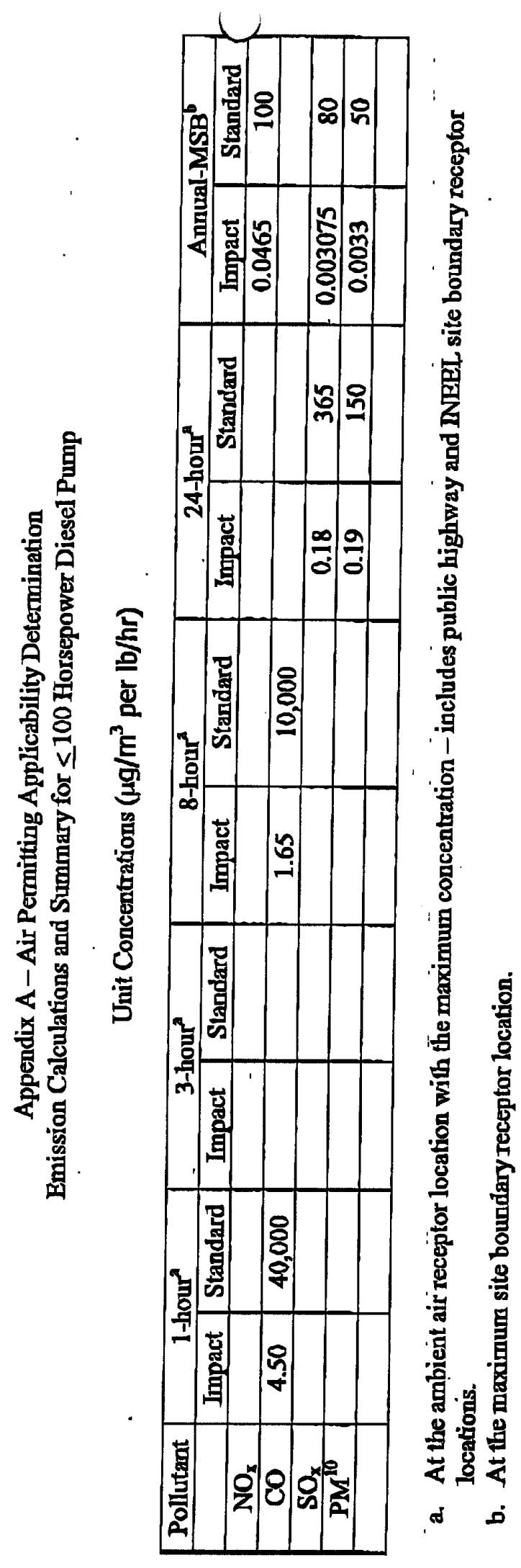




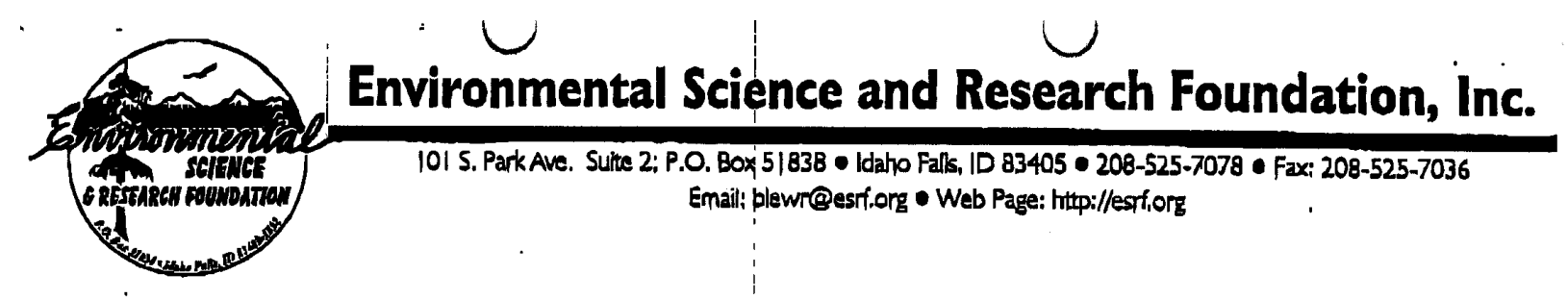

July 22, 1999

\begin{abstract}
Mr. Roger L. Twitchell
NEPA Compliance Officer

U. S. Department of Energy

Idaho Operations Office

850 Energy Drive, MS 1216

Idaho Falls, D' $83401-1563$
\end{abstract}

Subject: Ecological evaluation for revised location for the INTEC Percolation Pond Replacement Project (INTEC-99-008)

Dear Mr. Twitchell:

This letter provides recommendations in suppoirt of NEPA for activities related to the construction of two percolation ponds for disposal of service waste water at INTEC. The facility will also require two 14-inch pipes about 1,5 miles long extending from near the current percolation ponds to the new ones. This letter is in response to changes made in the siting of this facility.

The revised, proposed location for the ponds is southwest of INTEC (see attached map). The vegetation community of this area was classified as sagebrush steppe on lava and was dominated by big sagebrușh, green rabbitbrush, thickspike wheatgrass and bluebunch wheatgrass. $A$ total of 35 species of plants were noted in this area. Total plant cover was estimated at 25 percent. The soils were very gravelly. In this area, only one individual of the non-native species crested wheatgrass was found. This same community structure was found in the pipeline corridor from the ponds northeastward to just east of the bend in the corridor. At that point winterfat and crested wheatgrass also appeared as dominant species. Crested wheatgrass dominated the plant community from that point on east to INTEC.

The areas proposed for these activities are likely used by a diverse complement of small mammals, reptiles, and breeding bird species common to the sagebrush steppe. Some former Candidate species for listing as Threatened or Endangered (e.g, ferruginous hawk, loggerhead shrike and sagebrush lizard) are known to use these general areas. Big game animals likely using these areas include pronghorn anid mule deer.

Waste ponds are the only reliable water source on the INEEL and are used extensively by wildlife. The creation of ponds in this area will likely act as an attractant for wildlife, primarily waterfowl, pronghorn, mule deer, and potentially elk. These animals will likely increase use of this area because of the proposed water source.' 


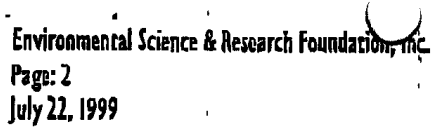

It is possible to design the proposed ponds to enhance wildlife habitat on the INEEL. To facilitate use by wildlife, the slopes of the berms should be as flat as possible and covered with native vegetation. Transfer pipes between cells should be screened to prevent waterfowl from entering. Lack of proper screens at other INEEL facilities has resulted in mortality of waterfowl protected by the Migratory Bird Treaty Act.

The project description does not mention the construction of fences around the percolation ponds. As long as contaminapt transport is not an issue, fences should not be installed. Fences themselves can often cause negative impacts to wildlife, especially big game. If the berm slopes are not too steep and are covered with vegetation, the risk of an animal becoming trapped in or near the water, as has happened at the INTEC sewage ponds, is small.

The project description does not mention returning vegetative cover to the pipeline corridor or to the berms around the ponds. We recommend planning for revegetation be completed prior to any excavation. Most of the soils in the INEEL have a horizon containing high concentrations of calcium carbopace starting at about $45 \mathrm{~cm}$ (eighteen inches) below the soil surface. This material is generally not suitable as topsoil because its high $\mathrm{pH}$ and low nutrient availability make it difficult to revegetate. The top $45 \mathrm{~cm}$ (eighteen inches) of soil should be reserved separate from the material below it as it is excavated. This reserved topsoil should be returned as the surface . horizon while backfilling the pipeline ditch, and spread over the surface of the berm around thie ponds. We recommend these areas be planted with native species. We believe this to be especially important for the pond area because non-native species are relatively rare in that area. The Foundation can provide assistance to the project manager in selecting appropriate species and seeding rates. To further reduce the costs and regulatory liabilities associated with revegetation, the area of soil disturbance should be kept as small as possible.

An additional consideration for revegetation on the pipeline corridor is the thermal load from the pipeline on the soil above it. The project manager should ensure that the amount of heat transferred to the overlying soil will not impede growth of vegetation.

An issue that is becoming increasingly important to ecological resources on the INEEL is fragmentation of habitat into smaller units. This project will expand the influence of the INTEC complex. Even though the percolation ponds cover only a few acres, they will likely affect the patterns of wildlife use over ain area larger than the present INTEC facility, To date, no assessment has been done of the cumulative impacts of such developments on ecological resources site-wide.

The areas likely to be affected by these activities are not wetlands, unique habitats, or areas known to be occupied by threatened or endangered species. It is unlikely the proposed activities will have any measurable impact on species of federal or state concern. It is my opinion a biological consultarion with the U.S. Fish and Wildlife Service is not necessary for these activities. 
Environmentad Sänce \& hesearch Foundationh m.

Page: 3

July 22, 1999

If you have any questions regarding this evaluation, please contact me at the lecterhead phone number.

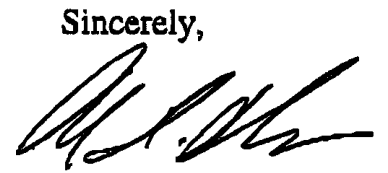

Roger D. Blew, Ph.D.

Plant Ecologist

Enclosure: As Stated

cc: - J. S. Irving, LMITCO, MS 3428

J. K. Winterholler, LMITCO, MS 5227

B. K. Evans, LMIICO, MS 4143 


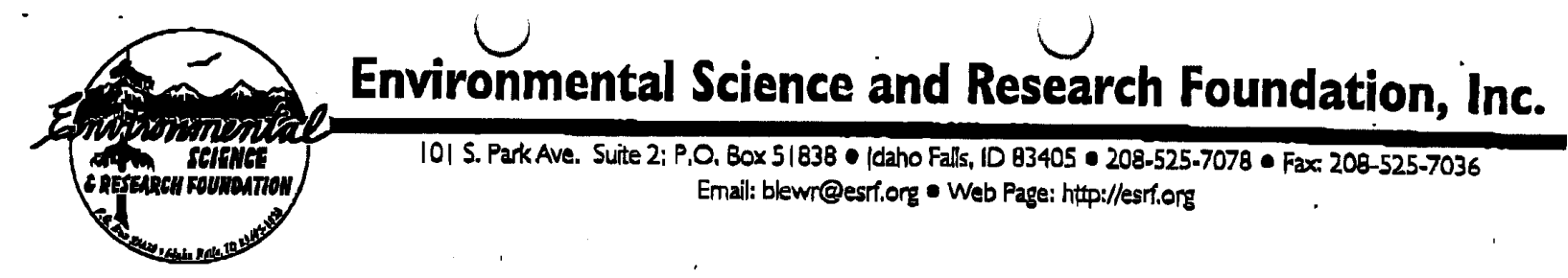

September 21, 1999

Mr. Roger L. Twitchell

NEPA Compliance Officer

U. S. Department of Energy

Idaho Operations Office

850 Energy Drive, MS 1216

Idaho Falls, ID 83401-1563

Subject: Ecological evaluation for revision to the INTEC Percolation Pond Replacement Project (INTEC-99-008)

Dear Mr. Twitchell:

This letter provides recommendations in support of NEPA for activities related to drilling monitoring wells associated with the INTEC Percolation Pond Replacement Project. A total of four wells will be drilled (see attached map).

The vegetation community of this area was classified as sagebrush steppe on lava and was dominated by big sagebrush, green rabbitbrush, thickspike wheatgrass and bluebunch wheatgrass. A total of 35 species of plants were noted in this area. Total plant cover was estimated at 25 percent. The soils were very gravelly. Two of the proposed well sites (002 and 003) are located in areas with significant densities of the non-native annual grass, cheatgrass.

The areas proposed for these activities are likely used by a diverse complement of small mammals, reptiles, and breeding bird species common to the sagebrush steppe. Some former candidate species for listing as threatened or endangered (e.g., ferruginous hawk, loggerhead shrike and sagebrush lizard) are known to use these general areas. Big game animals likely using these areas include pronghorn and mule deer.

The Foundation strongly suggests the size of the impacted area be kept as small as possible and weeds on the disturbed area, including cheatgrass, be controlled. All areas disturbed will require seeding with a mixture of native grasses. The Foundation also recommends that any roads built to the well pads be developed by mowing down the brush and the remaining vegetation not be grubbed, bladed or scraped off. Maintaining this native vegetation will aid in weed management on those roads. The Foundation can assist with details on revegetating the disturbed areas.

It is unlikely the proposed activities will have any measurable impact on species of federal or state concern. There are no federally listed or proposed threatened or endangered species, species of special concern, or records thereof, or designated critical habitat in proximity to the project 
Environmental Science \& Research Foundation, int.

Page: 2

September 21, 1999

area, the area of construction of storm water pollution prevention measures, the areas where storm water flows from the project area to the point of discharge, or in proximity to the area where storm water discharges into receiving waters. It is my opinion a biological consultation with the U.S. Fish and Wildlife Service is not necessary for these activities.

If you have any questions regarding this evaluation, please contact me at the letterhead phone number.

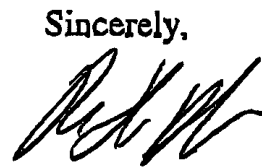

Roger D. Blew, Ph.D.

Plant Ecologist

Enclosure: As Stated

cc: J, S. Irving, LMITCO, MS 3428

J. K. Winterholler, LMTTCO, MS 5227

B. K. Evans, LMTTCO, MS 4143 
Environmental Science \& Research foundact d.

Page: 3

I

INTEC Percolation Ponds and Monitoring Wells

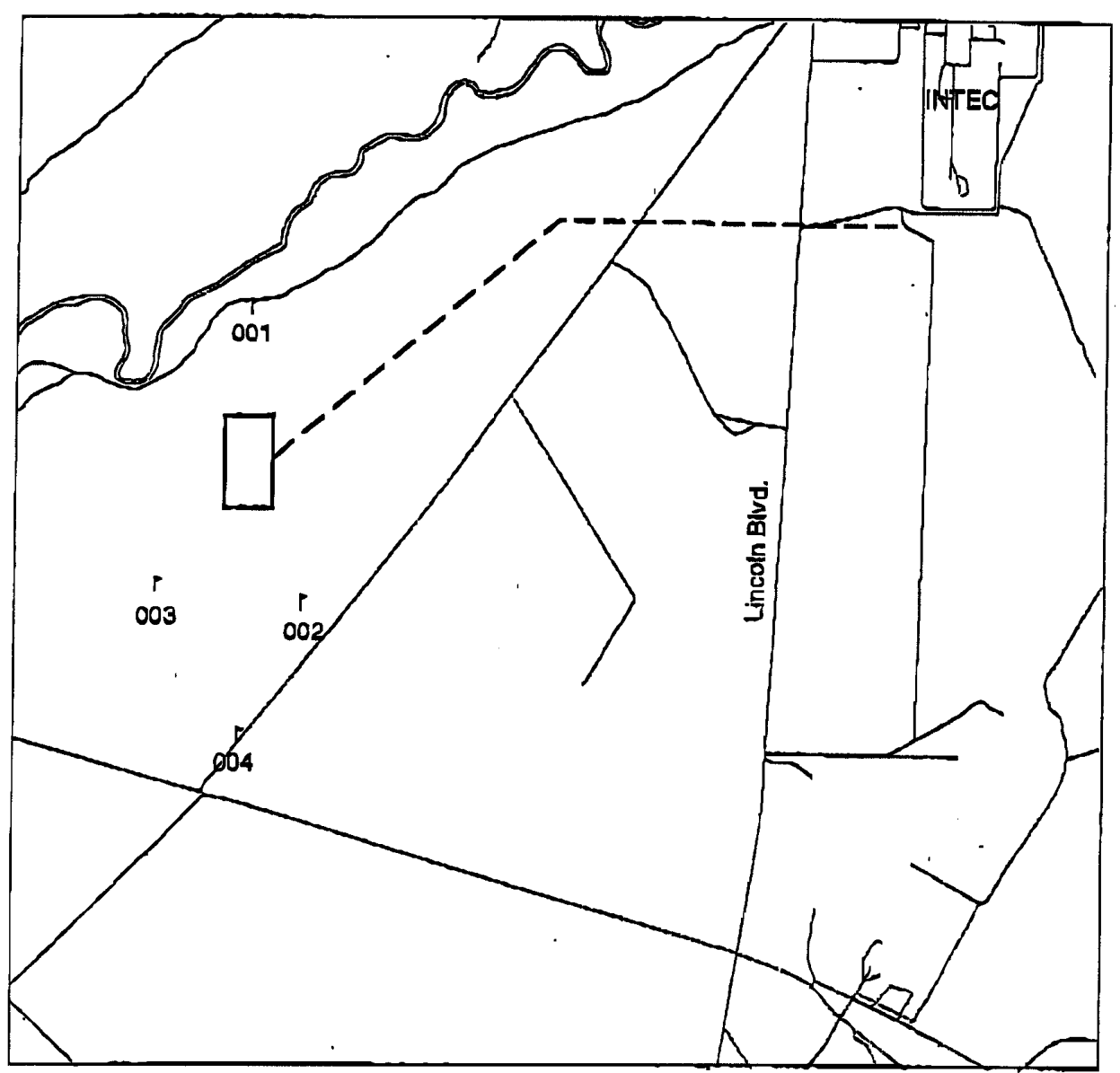

1/ Pipeline

Percolation Ponds

Proposed Monitoring Wells

$\checkmark$ Big Lost River

$\begin{array}{lllllll}0 & 0.2 & 0.4 & 0.6 & 0.8 & 1 & \text { Miles }\end{array}$

$\wedge$ Roads

Seplember 21, 1999 


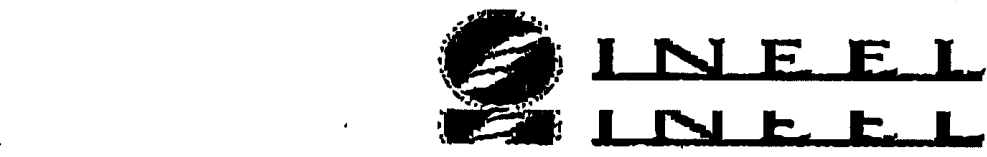

Brenda R Pace

09/15/99 11:23:31 AM

To: John K Winterholler/JKW/LMITCO/INEEL/US@INEL

cc: Brynna K Evans/EVANBK/LMITCO/INEEL/US@INEL, Clavton F Marler/CFM/LMITCO/INEELNS@INEL

Subject: Archaeological Clearance Recommendation

Hi Kirk, archaeological survey within the area surrounding the proposed new INTEC percolation ponds, pipeline, and associated wells is nearly complete. As of today, all currently proposed activity areas (ponds, pipeline/access road, four wells) have been intenelvely examined. Although several archaeological sites have been identified in the vicinity of these activity areas, I believe that all can be avoided by the work. Thls avoidance will require some coordination with the INEEL Cultural Resource Management Office. I will be pleased to provide this coordination for you, but will be out of the Office from September 17 through October 3, 1999. In my absence, Clavton Marler (6-0924) can assist you. When I return on October 4, 1999, I will assemble the Information from the most recent well surveys and complete the archaeological technical repor for the entire project. This shouldn't take long, and I expect that we can get it out to the State Historic Preservation Office and Shoshone-Bennock Tribes for their review by the second week in October. In the interim before we receive comments from these Agencles, it is imperative that wo avoid damage to all identified archeeological resources in the INTEC perc pond study area. Again, Clayton Marler and I will assist in this avoidance. If care is taken to avoid the sensitivo areas, clearance is recommended for the work. Don't hesitate to call me or Clayton if you have any questions, -Brende- 


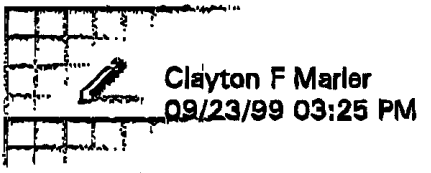

To: John K Winterholler/JKW/LMITCO/NEEL/US@INEL.

cC; Brynnạ K Evans/EVANBK/LMITCO/INEEL/US@INEL, Brenda A Paco/BXR/LMITCO/INEEL/US@INEL, Diana L Lowrey/DNO/LMITCO/NEEL/US@INEL

Subject: Percolation Pond distribution line

Kirk- we survejed the new east-west distribution line route yesterday and only encountered a small historic site. We surveyed a $50 \mathrm{~m}$ corridor on either side of the staked line yielding a total coverage of an approximate $100 \mathrm{~m}$ corridor. The historic site is located at the outer edge of the corridor, approximately $\mathbf{4 0} \mathrm{m}$ from the center and likely can be easily avoided. To aid avoidance measures flagging marking the site was left in place. With the exception of this site nothing was found and assuming that it can be avoided, cultural resource clearance is recommended for development work related to the new distribution line route. As she mentioned in her note, Brenda will complete. a more formal report for the entire project upon her return from Italy. If we can be of further assistance please let me know. Clayton 